Harald Schlee

\title{
Einkommensteuerliche Behandlung von Transferzahlungen
}




\section{Harald Schlee}

\section{Einkommensteuerliche Behandlung von Transferzahlungen}

Der Autor entwickelt ein in sich konsistentes System der Einkommensbesteuerung von Transferzahlungen, das vor allem an der Forderung nach Gleichmäßigkeit der Besteuerung orientiert ist. Zunächst werden allgemeine Grundsätze zur Steuerpflicht empfangener und geleisteter Transfers erarbeitet. Anschließend erfolgt eine intensive Auseinandersetzung mit Fragen der Besteuerung ausgewählter direkter staatlicher Transfers, der Alterssicherung und der Sicherung im Krankheitsfall sowie mit dem Gesamtkomplex der Familienbesteuerung. Das erarbeitete Reformkonzept beinhaltet zum Teil auch parallele Reformvorschläge für das Steuerrecht einerseits, das Sozial(versicherungs)recht und Zivilrecht andererseits.

Harald Schlee wurde 1958 in Frankfurt am Main geboren. Von 1978 bis 1983 Studium der Volkswirtschaftslehre an der Universität Frankfurt am Main. Anschließend war er Wissenschaftlicher Mitarbeiter am Institut für Finanzwissenschaft der Universität des Saarlandes (1985-1987) und am Institut für Öffentliche Wirtschaft. Geld und Währung der Universität Frankfurt am Main (1987-1992). Promotion 1993. 
Einkommensteuerliche Behandlung von Transferzahlungen

Zur Neuordnung der Familienbesteuerung sowie der Besteuerung von

Versicherungsleistungen und Sozialtransfers 


\section{FINANZWISSENSCHAFTLICHE SCHRIFTEN}

Herausgegeben von den Professoren

Albers, Krause-Junk, Littmann, Oberhauser, Pohmer, Schmidt

Band 56

\section{s \\ PETER LANG}

Frankfurt am Main - Berlin - Bern - New York - Paris - Wien

Harald Schlee - 978-3-631-75216-6

Downloaded from PubFactory at 01/11/2019 06:59:36AM

via free access 


\section{Harald Schlee}

\section{Einkommensteuerliche Behandlung von Transferzahlungen}

Zur Neuordnung der Familienbesteuerung sowie der Besteuerung von Versicherungsleistungen und Sozial transfers

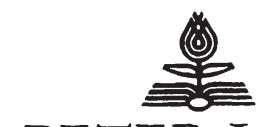

\section{PETER LANG}

Europäischer Verlag der Wissenschaften

Downloaded from PubFactory at 01/11/2019 06:59:36AM 
Die Deutsche Bibliothek - CIP-Einheitsaufnahme

Schlee, Harald:

Einkommensteuerliche Behandlung von Transferzahlungen : zur Neuordnung der Familienbesteuerung sowie der Besteuerung von Versicherungsleistungen und Sozialtransfers / Harald Schlee. - Frankfurt am Main ; Berlin ; Bern ; New York ; Paris ; Wien : Lang, 1994

(Finanzwissenschaftliche Schriften ; Bd. 56)

Zugl.: Frankfurt (Main), Univ., Diss., 1993

ISBN 3-631-46788-5

NE: GT

Open Access: The online version of this publication is published on www.peterlang.com and www.econstor.eu under the international Creative Commons License CC-BY 4.0. Learn more on how you can use and share this work: http://creativecommons.org/ licenses/by/4.0.

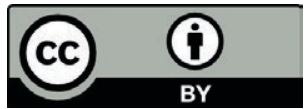

This book is available Open Access thanks to the kind support of ZBW - Leibniz-Informationszentrum Wirtschaft.

\author{
D 30 \\ ISSN 0170-8252 \\ ISBN 3-631-46788-5 \\ ISBN 978-3-631-75216-6 (eBook) \\ (C) Peter Lang GmbH \\ Europäischer Verlag der Wissenschaften \\ Frankfurt am Main 1994 \\ Alle Rechte vorbehalten.
}

Das Werk einschließlich aller seiner Teile ist urheberrechtlich geschützt. Jede Verwertung außerhalb der engen Grenzen des Urheberrechtsgesetzes ist ohne Zustimmung des Verlages unzulässig und strafbar. Das gilt insbesondere für Vervielfältigungen, Übersetzungen, Mikroverfilmungen und die Einspeicherung und Verarbeitung in elektronischen Systemen.

Printed in Germany $\begin{array}{r}1 \\ \text { Harald Schlee }\end{array}$ 
Justice is the first virtue of social institutions, as truth is of systems of thought.

(John Rawls, A Theory of Justice, Oxford u.a.O. 1973, 3) 
Harald Schlee - 978-3-631-75216-6

Downloaded from PubFactory at 01/11/2019 06:59:36AM

via free access 


\section{VORWORT}

Die vorliegende Arbeit entstand während meiner Tätigkeit am Institut für Finanzwissenschaft der Universität des Saarlandes sowie am Institut für Öffentliche Wirtschaft, Geld und Währung der Johann Wolfgang Goethe Universität Frankfurt am Main. Sie wurde am 17. Februar 1993 als Dissertation am Fachbereich Wirtschaftswissenschaften der Universität Frankfurt am Main angenommen. Die Arbeit war inhaltlich im Sommer 1992 weitgehend abgeschlossen, vor Drucklegung konnten die referierten gesetzlichen Regelungen allerdings noch auf den Stand vom 1.2.1993 gebracht werden.

Herrn Prof. Dr. Norbert Andel verdanke ich die Anregung zur Übernahme des Themas, vielfältige Anstöße aus der gemeinsamen Arbeit sowie zahlreiche kritische Anmerkungen zu einer ersten Fassung. Ihm und Frau Marlies Becker, Frau Susanne Gerecht und Frau Iris MüllerRohr möchte ich für die freundliche Aufnahme auf außerhessischem Gebiet danken, Frau Irmgard Benner, Frau Heike Beyer und Herrn Manfred Rosenstock für die langjährige gute Zusammenarbeit am Institut in Frankfurt. Schließlich bin ich Herrn Prof. Dr. Joachim Mitschke für die Übernahme des Korreferats und einige wichtige Verbesserungsvorschläge zu Dank verpflichtet.

Frankfurt am Main, im Juli 1993

Harald Schlee 
Harald Schlee - 978-3-631-75216-6

Downloaded from PubFactory at 01/11/2019 06:59:36AM

via free access 


\section{INHALTSÜBERSICHT}

$\begin{array}{ll}\text { I. Einleitung } & 1\end{array}$

II. Einkommensteuersystematische Grundlagen 13

III. Grundsätze der einkommensteuerlichen Behandlung von Transferzahlungen

IV. Direkte staatliche Transfers $\quad 61$

1. Sozialhilfe 61

2. Wohngeld und weitere wohnungsbezogene Transfers 67

3. BAföG-Leistungen 72

4. Kindergeld 75

V. Versicherungstransfers 77

1. Alterssicherung 77

2. Sicherung im Krankheitsfall 126

VI. Transfers zwischen Privaten, insbesondere innerfamiliäre Transfers 137

1. Zur Bedeutung der Familie für die individuelle soziale Sicherung

2. Haushalts- oder Individualbesteuerung? 140

3. Transfers zwischen Ehegatten (Ehegattenbesteuerung) 143

4. Allgemeiner Kinderlastenausgleich 175

5. Reduktion von Manipulationsmöglichkeiten 202

6. Versicherungen zugunsten Dritter 205

7. Sonderstatus für Erbschaften und Geschenke? 208

VII. Zusammenfassung und Ausblick 215 
Harald Schlee - 978-3-631-75216-6

Downloaded from PubFactory at 01/11/2019 06:59:36AM

via free access 


\section{INHALTSVERZEICHNIS}

\section{Einleitung}

1. Zielsetzung der Arbeit 1

2. Verwendeter Transferbegriff 4

3. Bewertungs- und Zurechnungsprobleme bestimmter Transferarten

3.1. Reale Transfers 7

3.2. Implizite Transfers 10

4. Gang der Untersuchung 11

\section{Einkommensteuersystematische Grundlagen}

1. Das Postulat der Besteuerung nach der Leistungsfähigkeit 13

2. Einkommen als Maßstab steuerlicher Leistungsfähigkeit 17

2.1. Der Einkommensbegriff der Reinvermögenszugangstheorie $\quad 17$

2.2. Einkommen als objektivierbare Größe 19

2.3. Einkommen als Ist-Einkommen 20

2.4. Einkommen als Entstehung von Eigentum 22

2.5. Einkommen als periodenbezogene Größe 23

2.6. Zur Gewährleistung der intertemporalen Vergleichbarkeit von Einkommen $\quad 26$

2.7. Einkommen als personenbezogene Größe 29

2.8. Zur Steuerfreiheit des Existenzminimums 30

2.9. Volkswirtschaftlicher vs. einkommensteuerlicher Einkommensbegriff 


\section{Grundsätze der einkommensteuerlichen Behandlung von Transferzahlungen}

1. Allgemeine Überlegungen

2. Zur Eignung des Korrespondenzprinzips als generelle Regelung

2.1. Definition 41

2.2. Interpersonales Korrespondenzprinzip 41

2.3. Intertemporales Korrespondenzprinzip 42

3. Überlegungen zu Sonderaspekten 45

3.1. Gefahrengemeinschaften $\quad 45$

3.2. Zwangsaspekte 48

3.3. Meritorische Aspekte 53

4. Methoden der steuerlichen Erfassung von Transferzahlungen 56

(1) Steuerpflicht 57

(2) Anrechnungsverfahren $\quad 57$

(3) Progressionsvorbehalt $\quad 58$

IV. Direkte staatliche Transfers

1. Sozialhilfe 61

1.1. Charakterisierung 61

1.2. Einkommensteuersystematische Aspekte 62

2. Wohngeld und weitere wohnungsbezogene Transfers 67

2.1. Charakterisierung 67

2.2. Einkommensteuersystematische Aspekte 68

3. BAföG-Leistungen $\quad 72$

3.1. Charakterisierung 72

3.2. Einkommensteuersystematische Aspekte 73

4. Kindergeld $\quad 75$

4.1. Charakterisierung 75

4.2. Einkommensteuersystematische Aspekte 76 


\section{Versicherungstransfers}

1. Alterssicherung 77

1.1. Charakterisierung 77

1.2. Einkommensteuersystematische Aspekte 79

1.2.1. Steuerfreiheit des Existenzminimums im Alter 79

1.2.2. Lebenseinkommensbesteuerung 81

1.2.3. Alterssicherung als meritorisches Gut 83

1.2.4. Zwang zur Alterssicherung 85

1.2.5. Schlußfolgerungen $\quad 86$

1.3. Sicherungsarten $\quad 86$

$\begin{array}{ll}\text { 1.3.1. Allgemeine Vermögensbildung } & 87\end{array}$

1.3.2. Kapitalversicherungen auf den Erlebensfall 90

1.3.3. Kapitalversicherungen auf den Todesfall 92

$\begin{array}{ll}\text { 1.3.4. Rentenversicherungen } & 94\end{array}$

1.3.4.1. Einkommensteuersystematische Einordnung 94

(1) Charakterisierung $\quad 94$

(2) Traditionelle Sicht: Leibrentenversicherung als Vermögensbildung $\quad 96$

(a) Normalbesteuerung 96

(b) Summarische Erfassung von Zinserträgen und
Umverteilungsgewinnen bei Auszahlung

(c) Ertragsanteilsbesteuerung 99

(d) Steuerstundung 101

(e) Anwendung des "Korrespondenzprinzips der nachgelagerten Art" 103

(f) Zusammenfassende Beurteilung 105

(3) Leibrentenversicherung als Transferinstitution 106

1.3.4.2. Gesetzliche Rentenversicherung 110

1.3.4.3. Private Rentenversicherung 115

$\begin{array}{ll}\text { 1.3.4.4. Beamtenversorgung } & 116\end{array}$

1.3.4.5. Betriebliche Altersversorgung 118

$\begin{array}{ll}\text { 1.3.4.6. Leibrentenvereinbarungen unter Privaten } & 120\end{array}$

1.3.5. Versicherungen zugunsten Dritter 122 
2. Sicherung im Krankheitsfall 126

2.1. Charakterisierung 126

2.2. Steuersystematische Aspekte 127

2.2.1. Sicherung gegen krankheitsbedingten Einkommensausfall 127

(1) Werbungskostencharakter der Aufwendungen 127

(2) Weitere Aspekte 128

2.2.2. Absicherung von krankheitsbedingten Kostenbelastungen $\quad 129$

(1) Sicherung des Existenzminimums 129

(2) Konsumelemente einer Krankenversicherung 130

(3) Meritorische Aspekte 131

(4) Zwang 131

(5) Intertemporaler Ausgleich 132

2.3. Umsetzung in die Steuerpraxis 132

2.4. Zum Verhältnis von Risikoselbsttragung und Versicherung 133

2.5. Besonderheiten der privaten Krankenversicherung 134

VI. Transfers zwischen Privaten, insbesondere innerfamiliäre Transfers

1. Zur Bedeutung der Familie für die individuelle soziale Sicherung

2. Haushalts- oder Individualbesteuerung? 140

3. Transfers zwischen Ehegatten (Ehegattenbesteuerung) 143

3.1. Grundsätzliche Problematik 143

(1) Rechtliche Ausgangssituation $\quad 143$

(2) Haushaltsersparnis und häusliche Dienste 146

(3) Aspekte der Steuerneutralität 146

3.2. Modelle der Ehegattenbesteuerung 148

3.2.1. Derzeitige Rechtslage 148

$\begin{array}{ll}\text { 3.2.2. Abgelehnte Alternativen } & 149\end{array}$ 
3.2.3. Befürwortete Alternativen $\quad 150$

3.2.3.1. Vorbemerkungen $\quad 150$

3.2.3.2. Grundformen 151

(1) Güterstand der Gütergemeinschaft 151

(2) Güterstand der Zugewinngemeinschaft 153

(3) Güterstand der Gütertrennung 155

(4) Familienphasenspezifische Regelung des Güterstandes?

3.2.3.3. Ergänzungen $\quad 159$

(1) Berücksichtigung der Haushaltsersparnis 159

(2) Berücksichtigung häuslicher Dienste 163

(3) Änderungen im Lohnsteuer-Abzugsverfahren zur Gleichstellung von Erst- und Zweitverdiener 167

3.2.3.4. Sonderfälle

(1) Zur Behandlung nichtehelicher Lebensgemeinschaften

(2) Zur Problematik von Änderungen in Lebensgemeinschaften

4. Allgemeiner Kinderlastenausgleich 175

4.1. Anforderungen 177

4.1.1. Steuersystematische Anforderungen 177

4.1.2. Sozialpolitische Anforderungen 180

4.1.3. Differenzierung nach Alter und Ordnungszahl der Kinder?

4.2. Unzulänglichkeiten des derzeitigen dualen Systems 183

4.3. Integrationskonzepte 185

4.3.1. Besteuerung des Kindergeldes 186

4.3.2. Konzeption der effektiven Gesamtentlastung 187

4.3.3. Bewertung und eigener Vorschlag 188

4.4. Zusätzliche Berücksichtigung des Kinderbetreuungs-
aufwands

$\begin{array}{ll}\text { 4.5. Zur Besteuerung der Kinder } & 198\end{array}$ 


\section{XVI}

5. Reduktion von Manipulationsmöglichkeiten 202

6. Versicherungen zugunsten Dritter 205

7. Sonderstatus für Erbschaften und Geschenke? 208

VII. Zusammenfassung und Ausblick 215

Literaturverzeichnis 221

$\begin{array}{ll}\text { Verwendete Gesetzestexte } & 241\end{array}$ 


\section{EINLEITUNG}

\section{Zielsetzung der Arbeit}

Ziel dieser Arbeit ist es, aufbauend auf dem normativen Postulat der Besteuerung nach der individuellen Leistungsfähigkeit, insbesondere der Gleichmäßigkeit der Besteuerung, Regeln für die einkommensteuerliche Behandlung von Transferzahlungen abzuleiten. Neben den genannten Gerechtigkeitsaspekten werden dabei in Form von Nebenzielen Transparenz-, Praktikabilitäts- und Allokationsaspekte sowie transferspezifische Zielsetzungen in umfassender Weise berücksichtigt und in bezug auf mögliche einkommensteuerliche Lösungen diskutiert.

Grundsätzlich kann davon ausgegangen werden, daß Steuergerechtigkeit nur innerhalb eines transparenten Steuersystems möglich ist, da sonst begünstigende Regelungen nur von einer kleinen Gruppe gut informierter Steuerpflichtiger in Anspruch genommen werden können. Auch sollten die Wirkungen des Steuersystems jedenfalls im Sinne der formalen Inzidenz für die Steuerpflichtigen überschaubar sein. Die Berücksichtigung des Praktikabilitätsziels erfordert ebenfalls eine Anpassung theoretischer Lösungen an die Bedingungen der Realität: Steuerliche Regelungen müssen so ausgestaltet werden, daß sie unter den in der Realität anzutreffenden Bedingungen mit einem vertretbaren Aufwand umgesetzt werden können. In beiden Fällen besteht daher eine ganz weitgehende Übereinstimmung mit dem Gerechtigkeitsziel. Zielkonflikte sind grundsätzlich zwischen dem Gerechtigkeitsziel und dem Ziel einer effizienten Faktorallokation zu erwarten, da letzteres keine subjektiven Aspekte berücksichtigt ${ }^{1}$. Sofern sich solche Zielkonflikte nicht ausräumen lassen, wird hier dem Gerechtigkeitsziel der Vorrang eingeräumt.

Es wird vom bestehenden deutschen Steuer- und Transfersystem mit seiner Vielzahl von eigenständigen Zielen und Instrumenten, privaten und öffentlichen Trägern ausgegangen. $\mathrm{Zu}$ untersuchen ist, wie die Ausgestaltung der Einkommensteuer so verändert werden kann, daß diese ihrer

1 Vgl. allerdings auch Kapitel II.1. 
zentralen Rolle als Leistungsfähigkeitssteuer und Umverteilungsinstrument besser gerecht wird, ohne dadurch andere eigenständige Zielsetzungen zu dominieren oder völlig auszuschalten. Insofern unterscheidet sich dieser Ansatz von Reformmodellen, die eine vollständige Integration von Steuer- und (staatlichem) Transfersystem vorsehen. Einerseits bleibt er hinter diesen Modellen zurück, da er nicht die Abschaffung eigenständiger Transferinstitutionen zum Ziel hat, andererseits geht er weit über diese hinaus, da er nicht nur staatliche Transfers zur Sicherung eines Grundbedarfs, sondern auch private Transferzahlungen und Zahlungen von (Sozial-)Versicherungen in die Betrachtung einbezieht. Gleichzeitig verbessert er jedoch auch die Ausgangsposition für eine Realisierung umfassender Integrationskonzepte im Grundbedarfsbereich.

Sofern die betrachteten Transferzahlungen auf eine Erhöhung (bzw. Minderung) individueller Leistungsfähigkeit ausgerichtet sind, ist deren einkommensteuerliche Relevanz offensichtlich. Gegebenenfalls ist hier auf unterschiedliche Leistungsfähigkeitsmaßstäbe im Einkommensteuerbereich einerseits, im Transferbereich andererseits und auf einzelne Mängel in der leistungsfähigkeitsbezogenen Ausgestaltung von Transferzahlungen hinzuweisen. Von besonderem Interesse sind transferrechtliche Regelungen des Sozial- und Zivilrechts, die einer einkommensteuersystematisch befriedigenden Lösung bei der Erfassung dieser Transfers entgegenstehen.

Verfolgen Transferzahlungen explizit eine objektbezogene Zielsetzung, was insbesondere bei Subventionen oft der Fall ist, lassen sich keine allgemein überzeugenden und befriedigenden Lösungen im Rahmen einer personenbezogenen Leistungsfähigkeitsbesteuerung ableiten. Es sind zwar Berührungspunkte für eine einkommensteuerliche Behandlung solcher Transferzahlungen vorhanden ${ }^{2}$, da die Ziele der Einkommensteuer und der betreffenden objektbezogenen Transfers jedoch auf unterschiedlichen Ebenen liegen, bleibt der Versuch einer integrierenden Sichtweise letztlich unergiebig.

2 Vgl. Kapitel III.3.3. 
Die Erfassung von Transferzahlungen steht in gewissem Widerspruch zur erwerbsorientierten Einkommensbesteuerung, wie sie heute üblich ist $^{3}$. Die in dieser Arbeit gemachten Vorschläge zur Transferbesteuerung zielen auch auf eine Erweiterung dieses Einkommensbegriffs, der einer umfassenden Ermittlung steuerlicher Leistungsfähigkeit im Wege steht, zumindest jedoch nicht dienlich ist. Dabei sind die Grenzen der Privatsphäre und die Praktikabilität von Neuregelungen zu beachten. Individuelle Persönlichkeitsrechte sind jedenfalls durch die Erfassung finanzieller Transaktionen durch gesetzlich dazu beauftragte Institutionen (Finanzbehörden) kaum berührt. Dies gilt insbesondere dann, wenn der Umfang der Informationen nicht wesentlich über die bereits heute im privaten Kreditgewerbe weitgehend unkontrolliert ausgetauschten Daten hinausgeht. Hinzu kommt, daß die Steuergesetzgebung an verfassungsrechtliche Regelungen gebunden ist, deren Einhaltung durch eine wirksame Kontrolle des Bundesverfassungsgerichts garantiert werden kann, so daß Befürchtungen vor einer konfiskatorischen Besteuerung unbegründet sind.

Wenn dem Vorschlag einer Erweiterung des bestehenden Einkommensbegriffs nicht gefolgt wird, lassen sich die hier für einzelne Arten von Transferzahlungen vorgeschlagenen einkommensteuerlichen Lösungen gleichwohl auch im Rahmen einer erwerbsorientierten Einkommensbesteuerung realisieren. Dann werden, wie heute bereits in Teilbereichen üblich, Transfereinkommen als aus Erwerbseinkommen abgeleitete Einkommen erfaßt. Als Problembereich verbleiben dann vor allem Erbschaften und Schenkungen aus vorhandenem Vermögen, die sich so nicht integrieren lassen.

Die hier vorgeschlagenen Lösungsansätze sind als Teil einer umfassenden Reformkonzeption der Einkommensteuer im Sinne der Reinvermögenszugangstheorie zu verstehen, wie sie in ihren Grundlinien in Kapitel II dargestellt wird. Da sie sich in ihrer Begründung immer wieder auf die in der Bundesrepublik Deutschland gegebenen institutionellen und

3 Vgl. Biergans/Stockinger (1982, 2-6), Kirchhof (1988, F20-F31) sowie Ruppe (1990, Rz.17). 
gesellschaftlichen Bedingungen beziehen ${ }^{4}$, können sie insofern keinen Anspruch auf Allgemeingültigkeit erheben.

\section{Verwendeter Transferbegriff}

Im folgenden lehne ich mich an eine Definition der Transfer-EnquêteKommission $(1981,22)$ an, die Transfers definiert als: "Alle Einkommen und einkommensähnliche Leistungen, die nicht Faktoreinkommen, und das heißt, Entgelt für die Nutzung von Produktionsfaktoren sind"5.

Die Transfer-Enquête-Kommission geht allerdings weder in ihrem Abschlußbericht noch in ihrem Zwischenbericht, in dem sie noch eine etwas andere Begriffsbildung vornimmt ${ }^{6}$, auf die Implikationen dieser Definition ein. Hier sollen deshalb ihre wichtigsten Eigenschaften - insbesondere auch im Vergleich zu anderen Definitionen - aufgezeigt werden:

Der Begriff "Einkommen und einkommensähnliche Leistungen" stellt direkt den Bezug zur Einkommensteuer her und ermöglicht daher für die Zwecke dieser Arbeit in idealer Weise die Anknüpfung an die Diskussion um die Bestimmung des steuerlichen Einkommensbegriffs ${ }^{7}$. Es werden sowohl monetäre wie nichtmonetäre Leistungen eingeschlossen, die aller-

4 Soweit im Einzelfall keine anderen Angaben gemacht werden, wird der Stand vom 1.2.1993 zugrundegelegt. Auf Übergangs- und Sonderbestimmungen für den Bereich der "neuen Bundesländer" wird nicht eingegangen.

5 Als Vorlage könnte der Kommission die Definition von Rolph (1948, 331) gedient haben, der Transfers definiert als "any income, either in money or in value in kind, accruing to persons or groups which is not in return for current services or products provided by them."

6 Transfers sind dort "Leistungsströme, denen keine spezielle Gegenleistung im Produktionsprozeß gegenübersteht und die kein Entgelt für die Nutzung von Produktionsfaktoren sind. Transfers lassen sich dann als die Übertragung von Ansprüchen auf Güter und Dienste außerhalb des marktmäßigen Verteilungsprozesses definieren." (Transfer-Enquête-Kommission, 1979, 36).

7 Vgl. die Ausführungen in Kapitel II. 
dings objektiv bewertbar sein müssen 8 . Eine Unterscheidung zwischen Einkommenstransfers und Vermögensübertragungen, wie sie in der Volkswirtschaftlichen Gesamtrechnung vorgenommen wird ${ }^{9}$, ist im Sinne der Reinvermögenszugangstheorie des Einkommens nicht angebracht; bei beiden Vorgängen handelt es sich für den Empfänger um Einkommen. Reine Vermögensumschichtungen können nach der gegebenen Definition nicht als Einkommen bezeichnet werden, der Ausdruck "rein intertemporale Transfers" wird somit als nicht sachgerecht abgelehnt 10 . Gleichwohl kommt der steuerlichen Behandlung dieser sog. "rein intertemporalen Transfers", also der Ersparnis, als Referenzmaßstab auch im Rahmen dieser Arbeit eine wichtige Bedeutung zul1.

Mit der negativen Abgrenzung "nicht Faktoreinkommen" werden alle Faktoreinkommen 12 - und lediglich diese - aus der Betrachtung ausgeschlossen. Oft anzutreffende unscharfe Begriffsbildungen wie "Leistungen ohne Gegenleistung" oder "one-way-transactions" werden vermieden 13: Neben Problemen der monetären Bewertung möglicher

8 Der Begriff "einkommensähnliche Leistungen" läßt zwar gerade in bezug auf die Bewertbarkeit einen gewissen Spielraum; dies bleibt aber für die Einkommensbesteuerung von Transfers ohne Folgen, da für die Einkommensteuer die dort übliche restriktivere Eingrenzung maßgeblich ist.

9 Vgl. Statistisches Bundesamt $(1989,92 \mathrm{f}$.) sowie Budd/Radner/Whiteman $(1984,39)$.

10 Zur Verwendung dieses Begriffs vgl. beispielsweise Lamers $(1975,77-79)$ sowie Kausemann (1983, 198f.).

11 Insbesondere auch bei Leistungsströmen, die sowohl einen Ersparnisanteil als auch einen - interpersonalen - Transferanteil enthalten. Zur Behandlung der Ersparnis vgl. Kapitel V.1.3.1.

12 Faktoreinkommen sind Entgelte für die Bereitstellung von Produktionsfaktoren. Hier werden auch die Unternehmergewinne als Faktoreinkommen betrachtet (vgl. dazu Stobbe, 1989, 83); andere Auffassungen finden sich in der älteren Literatur, so z.B. bei Rolph (1948, 332-344).

13 Vgl. Boulding (1973, 1): "one-way-transfer"; Mackscheidt (1983, 41): "Güter oder Geldströme, denen keine unmittelbaren Gegenleistungen entgegengesetzt sind"; Gandenberger (1989, 22): "Leistungen ohne Gegenleistung"; Busch (1975, 21): "keine marktliche Gegenleistung"; sowie Boulding/Pfaff (1972, 40): "Gegensatz zur vertragsbezogenen Tauschbeziehung". Daß mit Transfers sehr oft immaterielle Gegenleistungen verbunden sind, wird von Pfaff/Pfaff (1971, 280f.) betont. 
Gegenleistungen würde auf der Grundlage solcher Definitionen auch die Einbeziehung von Versicherungsleistungen zweifelhaft bleiben. Denn bei Versicherungsleistungen liegt eine Gegenleistung in Form von Beitragszahlungen vor, charakteristisch ist aber gerade, daß die in Aussicht gestellten Leistungen in der Regel vor dem Eintritt eines Versicherungsfalls nicht marktfähig und als tatsächliche Leistungen nicht ex ante bestimmbar sind, insofern also von Transfers gesprochen werden muß ${ }^{14}$. Nicht bestritten werden soll damit allerdings, daß auch eine definitorische Trennung von Markt- und Nicht-Markt-Vorgängen (Boulding/Pfaff, 1972, 40) bzw. von Tauschwirtschaft und "schenkender Wirtschaft" (Laum, 1960) sachgerecht sein kann, insbesondere um die Erweiterung des klassischen Untersuchungsobjekts der Wirtschaftswissenschaften aufzuzeigen.

Weiter kennzeichnet die hier verwendete Definition, daß sie keine institutionellen Eingrenzungen im Sinne Transferwirtschaft $=$ Staatswirtschaft vornimmt ${ }^{15}$. Aus ordnungspolitischer Sicht mag eine Trennung in Staatswirtschaft auf der einen, Privatwirtschaft auf der anderen Seite ihre Berechtigung haben, für die Zwecke dieser Arbeit, die Erfassung steuerlicher Leistungsfähigkeit beim Individuum, sind staatliche und private Transfers grundsätzlich wesensgleich.

14 Die schon traditionellen Schwierigkeiten bei Ein- oder Ausschluß von Versicherungstransaktionen werden deutlich bei Mackscheidt (1983, 42), der eine entsprechende Konvention betont, bei Gandenberger $(1989,22)$, der von "Quasitransfers" spricht, und bei Andel (1990, 222), der (Sozial-) Versicherungen nur wegen (ex ante!) Zwangs- und Umverteilungselementen einbezieht.

15 In der Literatur ist eine solche Eingrenzung des Untersuchungsobjekts oft anzutreffen, wenn auch z.T. dadurch begründet, daß nur bestimmte (staatliche) Institutionen den Untersuchungsgegenstand bilden. Vgl. z.B. Zeppernick (1986, 35-42), Busch $(1975,21)$, sowie Transfer-EnquêteKommission (1981, 3, Untertitel). In der allgemeinen Literatur zu privaten Transfers steht oft die Motivforschung im Vordergrund (vgl. Boulding, 1973, 15-34, und Rippe, 1981). 


\section{Bewertungs- und Zurechnungsprobleme bestimmter Transferarten}

In der Literatur wird i.d.R. zwischen monetären und realen Transfers, expliziten und impliziten Transfers sowie direkten und indirekten Transfers unterschieden, wenn die Wirkungen (staatlicher) Transferleistungen auf die personale Einkommensverteilung untersucht werden 16 .

Im späteren Verlauf der Arbeit wird auf die mit diesen Klassifikationen angesprochenen qualitativen Unterschiede von Transferleistungen nicht mehr eingegangen, stattdessen als Voraussetzung einer einkommensteuerlichen Erfassung davon ausgegangen, daß Bewertungs- und Zurechnungsprobleme gelöst sind, wie dies für explizite direkte monetäre Transfers unterstellt werden kann. Daher ist an dieser Stelle auf die besondere Problematik realer und impliziter Transfers einzugehen. Indirekte Transfers werden nicht näher behandelt, weil Fragen des Wirkungsverlaufs indirekter Transfers 17 hier ungeklärt bleiben können. Für Zwecke der Einkommensbesteuerung kommt es allein auf den Zufluß beim letztlich Begünstigten an 18 .

\subsection{Reale Transfers}

Im Gegensatz zu monetären Transfers ergibt sich bei realen Transfers, also Transfers in Form von Gütern ${ }^{19}$, zwangsläufig ein Bewertungs-

16 Vgl. Kammann (1980, 8-43), Transfer-Enquête-Kommission (1981, 22-24) sowie Zeppernick (1986, 42-62).

17 Vgl. dazu Andel (1977, 501-503).

18 Sofern dieser sich in Form erhöhter Faktorentgelte ausdrückt, läßt er sich einkommensteuerlich gut erfassen. Es verbleiben allerdings Probleme der Berücksichtigung selektiver Preisänderungen im Konsumgüterbereich.

19 Gegebenenfalls ist die Differenz zwischen (Markt-)Wert und gezahltem Entgelt als Realtransfer zu werten. Für allgemeine Ausführungen zu Realtransfers vgl. Zeppernick (1986, 44-55) und Transfer-EnquêteKommission (1979, 40-42; 1981, 62-81). 
problem ${ }^{20}$. Orientierungspunkt für die Bewertung realer Transfers sollte die individuelle marginale Wertschätzung des Transferempfängers sein 21 . Da diese für Zwecke der Besteuerung praktisch nicht zu ermitteln ist (bei Enthüllung der Präferenzen drohen die negativen Konsequenzen einer Steuerzahlung), bieten sich für eine hilfsweise Bewertung, die allgemein akzeptiert werden kann, vor allem Marktpreise an ${ }^{22}$. Insbesondere bei öffentlich bereitgestellten Gütern fällt auch diese Möglichkeit i.d.R. aus, so daß nur eine Bewertung zu Erstellungskosten bleibt 23 . Eine solche Vorgehensweise ist sehr problematisch:

Bei (reinen) öffentlichen Gütern läßt sich ein Zuwachs an ökonomischer Dispositionskraft für den Kreis der potentiellen Nutzer nicht unterstellen ${ }^{24}$, da die individuellen Präferenzen unbekannt bleiben und eine Marktfähigkeit nicht gegeben ist. Auch kann die Bereitstellung öffentlicher Güter, durch die keine Begünstigung oder Diskriminierung bestimmter Personen erfolgt, als Grundversorgung eingestuft werden, die dann als Bestandteil eines steuerfreien Existenzminimums kein̉en Verstoß gegen den Grundsatz der horizontalen Steuergerechtigkeit hervorruft 25 .

20 Dies gilt nicht für die Sachleistungen der Krankenversicherungen, die sowohl Eigenschaften monetärer als auch realer Transfers aufweisen: Die Beziehung Krankenversicherung zu Versicherten läßt sich als Kostenerstattungsabkommen interpretieren, so daß sich sowohl die Bewertung als auch die individuelle Zurechnung der Leistungen klären lassen. Andererseits bleiben Probleme, die sich aus einer kostenlosen Bereitstellung ergeben und für reale Transfers typisch sind, erhalten. Vgl. TransferEnquête-Kommission (1979, 41f.), Lukarsch (1988, 960).

21 Vgl. Prest $(1968,86 f$.$) , Smeeding (1984,145 f$.) sowie OECD (1988a, 16f.).

22 Hier ist - z.T. sehr folgenreich - zwischen Kauf- und Verkaufspreis zu differenzieren: Eine höhere Wertschätzung als die des Verkaufspreises kann nicht sicher unterstellt werden (OECD, 1988a, 17).

23 Grünärml/Henke $(1973 / 74,251)$ halten eine solche Vorgehensweise für vertretbar. Kritisch hierzu Smeeding (1984, 141-147).

24 Vgl. Zeppernick (1986, 45). Anderer Ansicht ist Mackscheidt (1976, 6874), der nahezu die gesamte öffentliche Leistungserstellung als gruppenspezifisch einstuft.

25 Vgl. indirekt hierzu Hackmann $(1983,687)$. 
Bei meritorischen Gütern, bei denen eine individuelle Zuordnung eher vorgenommen werden kann, kommt erschwerend hinzu, daß oft bereits von der Konzeption her die Wertschätzungen von Transfergeber und Transferempfänger auseinanderfallen (Pommerehne, 1979, 149-151). Wäre dies nicht der Fall, dann könnten im allgemeinen bei Wohlfahrtsgewinnen für die Empfänger Realtransfers durch monetäre Transfers ersetzt werden (Browning, 1975, 541-543). Da das Ziel i.d.R. eine möglichst große Inanspruchnahme dieser Leistungen ist (im Extremfall unter Anwendung von Zwang wie bei der Schulpflicht), würde eine Besteuerung zu unerwünschten Nachfrageeinschränkungen führen.

Über die private Wertschätzung dieser Leistungen läßt sich daher sicher nur sagen, daß sie - kostenlose Bereitstellung und Zurückweisbarkeit der Leistungen vorausgesetzt - größer als Null ist. Eine Besteuerung würde die Inanspruchnahme einschränken, da in diesem Fall eine Wertschätzung mindestens in Höhe der Steuerbelastung vorliegen muß.

Es ergibt sich ein steuerpolitisches Dilemma: Die vorgetragenen Argumente sprechen gegen eine Steuerpflicht von staatlichen Realtransfers - jedenfalls soweit sie sich nicht zu Marktpreisen weiterveräußern lassen; andererseits bedeutete ein Verzicht auf Besteuerung wegen des dann vorhandenen Steuervorteils eine tendenzielle Verschiebung der Bürgerpräferenzen hin zu realen Transfers, obwohl diese im allgemeinen mit einem Wohlfahrtsverlust verbunden sind.

Als Lösung bietet sich nur eine Umwandlung realer in nicht zweckgebundene monetäre Transfers bzw. eine Erhebung kostendeckender Gebühren an, sofern es sich nicht um die Bereitstellung einer Grundversorgung handelt oder gewichtige meritorische Aspekte dagegen sprechen.

Die in der Literatur genannten hohen Summen, die für öffentliche Realtransfers aufgewendet werden (Zeppernick, 1986, 46) legen den Schluß nahe, daß es sich hier um ein zentrales Problem der Transferbesteuerung handeln müsse. Dies ist jedoch nicht der Fall, da für öffentliche Güter die dargestellten Überlegungen einer Grundversorgung zutreffen, bei den individuell zurechenbaren Leistungen die immer wieder genannten Bereiche der gesetzlichen Krankenversicherung, des sozialen 
Wohnungsbaus und der öffentlich finanzierten Ausbildungsleistungen 26 nicht so problematisch sind, wie sie auf den ersten Blick scheinen: Jedenfalls bei Sachleistungen der gesetzlichen Krankenversicherungen kann i.d.R. nicht von einer Erhöhung der steuerlichen Leistungsfähigkeit gesprochen werden, stattdessen sind sie als Bestandteil des (steuerfreien) Existenzminimums zu betrachten 27 . Bei Sozialwohnungen lassen sich Marktmieten vergleichbarer Wohnungen recht gut feststellen 28 . Ausbildungsleistungen werden, soweit sie zu höheren individuellen Einkommen führen, dann mit Verzögerung einkommensteuerlich erfaßt, auch wenn Humankapital nicht zu aktivieren ist (Andel, 1980, 365f.).

\subsection{Implizite Transfers}

Implizite Transfers sind dadurch gekennzeichnet, daß sie nicht in offener Form ausgewiesen werden. Sie bestehen in einem Einnahmeverzicht, der nur als Abweichung von einer Norm festgestellt werden kann; bei dieser Norm handelt es sich i.d.R. im privaten Bereich um einen Marktpreis, im staatlichen Bereich um eine Steuernorm 29.

Während im privaten Bereich die Feststellung von Marktpreisen noch relativ unproblematisch ist (üblicher Verkaufspreis, Marktzins für Kredite bestimmter Laufzeit), bereitet die Festlegung einer allgemein akzeptierten

26 Vgl. Transfer-Enquête-Kommission (1979, 42; 1981, 62-81) sowie Zeppernick $(1986,50)$.

27 Vgl. Kapitel V.2.2.2.

28 Wegen der verhältnismäßig homogenen Präferenzen am Wohnungsmarkt, insbesondere bei Wohnungen einfacher Ausstattung, lassen sich die Marktmieten hier als gute Näherung für die Wertschätzung von Sozialmietern heranziehen.

29 Vgl. Busch $(1975,12,20)$, Transfer-Enquête-Kommission (1979, 42f.) sowie Zeppernick (1986, 55-60). Eine weitere Abgrenzung des Begriffs der impliziten Transfers findet sich bei Boulding (1973, 49-61). Hier werden als implizite Transfers auch alle indirekten Begünstigungen durch Normsetzungen des Staates erfaßt. Dies ist aber nicht mehr handhabbar und führt in die Richtung, allgemeine ökonomische Interdependenzen zu berücksichtigen (vgl. dazu Schlieper, 1980, 524). 
Steuernorm erhebliche Schwierigkeiten 30 . Wie die Höhe der impliziten staatlichen Transfers von der zugrundegelegten Norm abhängt, zeigt das Beispiel des Ehegattensplitting: Im Sozialbericht (Bundesminister für Arbeit und Sozialordnung, 1990, 135, Ziff.34) wird es als sozialpolitische Leistung aufgeführt, im Subventionsbericht (Bundesministerium der Finanzen, 1991) jedoch nicht als Steuervergünstigung.

Staatliche implizite Transfers in Form von Steuervergünstigungen sollten immer dann, wenn sie nicht Bestandteil einer umfassenden Leistungsfähigkeitssteuer (i.d.R. der Einkommensteuer) sind, zur Erhöhung der Transparenz in offene Transfers umgewandelt werden (Busch, 1975, 18). Grundsätzlich sind implizite Transfers nach den gleichen Regeln wie explizite Transfers der Einkommensteuer zu unterwerfen.

\section{Gang der Untersuchung}

Nachdem eine Abgrenzung des zugrundegelegten Transferbegriffs bereits in Kapitel I vorgenommen wurde, werden in Kapitel II die einkommensteuersystematischen Grundlagen der Arbeit gelegt. Zunächst wird die Orientierung am Leistungsfähigkeitsprinzip in der speziellen Ausprägung des Grundsatzes der Gleichmäßigkeit der Besteuerung ausführlich diskutiert und begründet. Auf der Basis der Reinvermögenszugangstheorie werden anschließend einige Implikationen des steuerlichen Einkommensbegriffs aufgezeigt, die im Rahmen dieser Arbeit von besonderer Bedeutung sind.

In Kapitel III werden, aufbauend auf den vorangegangenen steuersystematischen Überlegungen, zunächst allgemeine Grundsätze der einkommensteuerlichen Behandlung von Transferzahlungen abgeleitet. Dabei erfolgt auch eine Erörterung der Eignung des Korrespondenzprinzips als generelle Regel. Sodann werden diese Überlegungen durch eine Berücksichtigung häufig auftretender besonderer Rahmenbedingungen von Transferzahlungen (Gefahrengemeinschaften, Zwangsaspekte, meritorische Aspekte) modifiziert. Eine Auseinandersetzung mit

30 Auch die vorliegende Arbeit beschäftigt sich mit diesem Thema. 
verschiedenen Methoden der steuerlichen Erfassung von Transferzahlungen schließt das Kapitel ab.

In den folgenden Kapiteln IV - VI wird auf die spezielle Ausgestaltung von Transferzahlungen in der Bundesrepublik Deutschland eingegangen. Für ausgewählte Arten von Transferzahlungen werden einkommensteuerliche Lösungen entwickelt, die den jeweiligen institutionellen Besonderheiten Rechnung tragen und sich gleichwohl in ein Gesamtkonzept einfügen. Die jeweiligen Begründungslinien werden offengelegt und ausführlich diskutiert, insbesondere auch unter Einbeziehung sozialpolitischer und rechtlicher Aspekte. Sofern die betrachteten institutionellen Regelungen eine in sich konsistente Ausgestaltung vermissen lassen, was auch einkommensteuersystematisch befriedigende Lösungen behindert, werden ebenfalls Ansätze zu deren Reform aufgezeigt.

Unterschieden werden dabei direkte staatliche Transfers (Kapitel IV), Versicherungstransfers (Kapitel V) und Transfers zwischen Privaten, insbesondere innerfamiliärer Art (Kapitel VI). Schwerpunkte bilden in Kapitel IV die Sozialhilfe, in Kapitel V die Alterssicherung sowie die Sicherung im Krankheitsfall und schließlich im Rahmen der Behandlung innerfamiliärer Transfers in Kapitel VI die Ehegattenbesteuerung und der Kinderlastenausgleich. Damit werden die jeweils wichtigsten Arten von Transfers explizit behandelt; implizit ergeben sich daraus auch die Grundlinien einer Reform für nicht gesondert angesprochene Transferarten.

In Kapitel VII wird ein Gesamtüberblick über die vorgeschlagenen Reformen gegeben. Schließlich werden in Form eines Ausblicks die Bedingungen für eine Weiterentwicklung des Einkommensteuersystems im hier vorgeschlagenen Sinne näher betrachtet. 


\section{EINKOMMENSTEUERSYSTEMATISCHE GRUNDLAGEN}

\section{Das Postulat der Besteuerung nach der Leistungsfähigkeit}

Das Postulat der Besteuerung nach der Leistungsfähigkeit beinhaltet die Forderungen nach Allgemeinheit, nach Gleichmäßigkeit und nach Verhältnismäßigkeit der Besteuerung. Nach dem Grundsatz der Allgemeinheit sollen alle über steuerliche Leistungsfähigkeit verfügenden Personen zur Besteuerung herangezogen werden; nach dem Grundsatz der Gleichmäßigkeit sollen Personen mit gleicher steuerlicher Leistungsfähigkeit, also in gleichen steuerlich relevanten Verhältnissen, auch gleich hohe Steuern zahlen; nach dem Grundsatz der Verhältnismäßigkeit soll die Differenzierung der Steuerlast den Unterschieden der individuellen Leistungsfähigkeiten Rechnung tragen 1 .

Diese Arbeit befaßt sich vor allem mit Fragen der Gleichmäßigkeit der Besteuerung, die auch als Fragen der "horizontalen Gerechtigkeit" bezeichnet werden ${ }^{2}$, d.h. mit der umfassenden Ermittlung der durch die Steuerbemessungsgrundlage repräsentierten steuerlichen Leistungsfähigkeit. Nach meinem Verständnis schließt die Forderung nach Gleich-

1 Vgl. Neumark (1970, 74-185; 1977, 303f.). Neumark nennt in beiden Publikationen noch den Grundsatz der steuerlichen Umverteilung, der eine stärkere Tarifprogression als nur eine solche, die zu relativ gleichen Änderungen der Dispositionskraft der Steuerpflichtigen führt, fordert. Dieses Postulat steht in enger Beziehung zum Grundsatz der Verhältnismäßigkeit und ist in der Praxis der Steuerpolitik untrennbar mit diesem verbunden (Neumark, 1970, 69). Mit einem zusätzlichen "Grundsatz der Vermeidung von ungewollten inflationsbedingten Mehrbelastungen und von Verzerrungen der Belastungsproportionen" (Neumark, 1977, 304) wird ein spezielles Problem der korrekten Ermittlung der steuerlichen Bemessungsgrundlage angesprochen, das in dieser Arbeit später gesondert behandelt wird (vgl. Kapitel II.2.6).

2 Abweichend von der Neumarkschen Terminologie findet sich in der Literatur die Unterscheidung in horizontale Gerechtigkeit und vertikale Gerechtigkeit (Musgrave/Musgrave/Kullmer, 1988, 18). Die Forderung nach horizontaler Gerechtigkeit entspricht dabei den oben genannten Grundsätzen der Allgemeinheit und Gleichmäßigkeit, die nach vertikaler Gerechtigkeit dem Grundsatz der Verhältnismäßigkeit. 
mäßigkeit die Forderung nach Allgemeinheit mit ein und ist selbst Voraussetzung für die Verwirklichung der Forderung nach Verhältnismäßigkeit ${ }^{3}$.

Während sich das Postulat der Verhältnismäßigkeit der Besteuerung einer allgemeinverbindlichen Konkretisierung entzieht ${ }^{4}$, daher z.T. auch als inhaltsleer bezeichnet wird (Littmann, 1970, 113), kann das Postulat der Gleichmäßigkeit der Besteuerung innerhalb der traditionellen Finanzwissenschaft auf breite Zustimmung rechnen 5 . Voraussetzung ist allerdings, daß die sich am Markt ergebenden Einkommen als Ausgangsbasis einer gerechten Besteuerung akzeptiert werden (Gordon, 1972, 321).

So steht denn auch als Ziel der Steuerreformen der letzten Jahre verbunden mit einer Senkung der marginalen Steuersätze - die umfassende Berücksichtigung der steuerlichen Leistungsfähigkeit, die Verwirklichung einer "comprehensive tax base" im Vordergrund6.

Gegen eine solche umfassende Konzeption wird eingewandt, die real existierenden Steuersysteme seien so weit vom Ideal einer umfassenden Bemessungsgrundlage entfernt, daß lediglich Ad-hoc-Entscheidungen gefragt seien, eine Orientierung an einer ohnehin visionären Vorstellung

3 Vgl. zur Darlegung der Beziehungen zwischen den Neumarkschen Gerechtigkeitspostulaten Koch $(1966,319)$ sowie Neumark $(1970,69 f$.$) . Die$ Auswirkungen einer Verletzung des Postulats der Gleichmäßigkeit auf die vertikale Steuerlastverteilung illustriert Pechman $(1986,74,89-95)$ am Beispiel der steuerlichen Erfassung von "capital gains" besonders deutlich.

$4 \mathrm{Vgl}$. zu der Variationsbreite der aus gängigen Nutzenfunktionen ableitbaren Steuertarife Hinterberger/Müller/Petersen (1987). Der Steuertarif ist daher vor allem Ausdruck gesellschaftlicher Werturteile (Neumark, 1961a, 373f.).

$5 \mathrm{Vgl}$. insbesondere auch Littmann $(1970,132)$, der einen "allgemeinen Gleichheitssatz" einführt.

6 Vgl. z.B. President of the United States (1985, Summary, 1-30). Auch in Deutschland hat dieses Ziel in der Steuerreformdiskussion einen hohen Stellenwert; in der Praxis der "Steuerreform 1990" hat es sich allerdings nur in relativ geringem Ausmaß niedergeschlagen. Vgl. dazu den Katalog "Abbau von Steuervergünstigungen und Sonderregelungen für ein gerechteres und einfacheres Steuersystem" (Bundesministerium der Finanzen, 1987, 50-56). 
keinen Gewinn bringe7. Dagegen kann angeführt werden, daß zwar im Durchschnitt der Steuerpflichtigen die Verteilungswirkungen von Steuervergünstigungen ${ }^{8}$ gegeneinander abgewogen werden können, aber nur die Verwirklichung einer umfassenden Bemessungsgrundlage Steuergerechtigkeit im Einzelfall sicherstellt (R.A.Musgrave, 1968a, 67-69; R.A.Musgrave, 1968b, 114-116) sowie ein Höchstmaß an individueller Wahlfreiheit garantiert (Hettich, 1979, 4-7). Darüber hinaus reduziert eine möglichst umfassende Bemessungsgrundlage durch eine Minimierung der erforderlichen Steuersätze (bei gegebenem Steueraufkommen) noch verbleibende steuerliche Ungerechtigkeiten, während bei einem Abgehen von diesem Konzept durch notwendigerweise höhere Steuersätze insoweit ein größeres Ausmaß an steuerlicher Ungerechtigkeit die Folge sein müßte (Brennan, 1971, 444-450).

Ein anderer Einwand lehnt überhaupt die Zugrundelegung eines Postulats der steuerlichen Gerechtigkeit ab. Ein solches Postulat bringe Werturteile in die ökonomische Analyse mit ein, die sich der wissenschaftlichen Diskussion entziehen. Stattdessen wird als Ziel einer "Optimal Income Taxation" die Maximierung einer sozialen Wohlfahrtsfunktion unter expliziter Berücksichtigung von Arbeitsangebotsreaktionen vorgeschlagen ${ }^{9}$. Es kann allerdings davon ausgegangen werden, daß ein derartiges allein am Effizienzziel ausgerichtetes Steuersystem wegen mangelnder Gleichmäßigkeit der Besteuerung 10 von den Steuerpflichtigen als ungerecht empfunden wird und daher negative steuerliche und darüber hinaus gesellschaftspolitische Reaktionen auslöst: Auf die rein steuerlichen Reaktionen verweisen verschiedene Arbeiten, die sich mit den

7 Vgl. zu solchen Einwänden Bittker $(1968,59 \mathrm{f}$ ), in abgemilderter Form: Hettich (1983).

8 Als Steuervergünstigungen werden steuersystematisch nicht angebrachte Abweichungen von der Konzeption der umfassenden Bemessungsgrundlage, die als Norm gesetzt wird, verstanden.

9 Vgl. dazu von Oehsen (1982, 231-233) sowie die kritische Position von Head (1982, 197-199).

10 Zur Unvereinbarkeit der Ziele Wohlfahrtsmaximierung und Gleichmäßigkeit der Besteuerung vgl. Stiglitz (1982). 
Determinanten der Bereitschaft zur Steuerhinterziehung befassen 11. Auch wird der hohe Wert, der der gesellschaftlichen Akzeptanz eines Steuersystems (und damit der Steuergerechtigkeit) als Teil eines Sozialvertrags 12 zukommt, von der utilitaristisch ausgerichteten Literatur i.d.R. unterschätzt. Darüber hinaus würde eine konsequente Ausrichtung an den Konzepten der Optimal-taxation-Theorie in einem solch großen Ausmaß die Kenntnis persönlicher Daten erfordern, wie es in Ansätzen allenfalls unter hohen Erhebungskosten und - was mir erheblich wichtiger erscheint - für totalitäre Staaten vorstellbar ist 13 .

Weiter ist der oben bereits unter Gerechtigkeitsgesichtspunkten genannte Aspekt einer möglichen Minimierung der Steuersätze durch eine Ausweitung der steuerlichen Bemessungsgrundlage auch unter Effizienzaspekten bedeutsam, da die durch die Besteuerung hervorgerufenen Verzerrungen der Ressourcenallokation - u.a. auch indirekt durch die lohnendere Suche nach Steuervermeidungsmöglichkeiten - mit zunehmendem Steuersatz überproportional ansteigen 14 .

Das Ziel der Gleichmäßigkeit der Besteuerung bildet daher die normative Grundlage dieser Arbeit. Bei der Umsetzung in konkrete Steuerreformvorhaben sind allerdings Übergangsprobleme zu beachten ohne daß Besitzstandswahrung ein so restriktiver Faktor sein sollte, wie

11 Vgl. Gaertner $(1988,123,125)$, Schlicht $(1985,265-271)$ sowie Lewis (1982, 182f.).

$12 \mathrm{Zu}$ Kriterien, die in einer konstitutionellen Phase für die Wahl eines Steuersystems ausschlaggebend sein können vgl. die Überlegungen von Buchanan $(1967,213-255)$ und Walzer $(1987,107-119,146-166)$.

13 Vgl. Seidl (1983, 207-210), Meade (1978, 25-27) sowie Head $(1982,198)$. Wird dagegen von der Verwendung individueller Daten abgegangen, so verliert das Konzept der Optimal taxation bei Vorliegen inhomogener Präferenzen der Wirtschaftssubjekte bereits aus sich heraus an Aussagekraft.

14 Vgl. Hotelling $(1969,287)$ und Harberger $(1974,40-47)$. Dem widerspricht die Forderung von Brennan/Buchanan (1980, 34-54), als Schutz der Bürger vor einem allmächtigen Staat Lücken in der steuerlichen Bemessungsgrundlage zu lassen. Selbst wenn man dieser Sichtweise zustimmt, läßt sich der gleiche Effekt aber auch durch eine Begrenzung der zulässigen Steuersätze erzielen (vgl. Brennan/Buchanan, 1980, 34-54, sowie Head, 1982, 196). 
Feldstein das nahelegt 15 . Auch ist gegebenenfalls in Erwägung zu ziehen, globale Änderungen der Steuerlastverteilung durch Anpassungen im Steuertarif auszugleichen 16 .

\section{Einkommen als Maßstab steuerlicher Leistungsfähigkeit}

\subsection{Der Einkommensbegriff der Reinvermögenszugangstheorie}

Der hier verwendete Einkommensbegriff ist jener der Reinvermögenszugangstheorie, der auf Schanz zurückgeht und im angelsächsischen Sprachraum vor allem von Haig und Simons eingeführt wurde (daher auch die Bezeichnung SHS-Konzept). Gemeinsam ist diesen drei Autoren die Suche nach einem Einkommensbegriff, der möglichst umfassend den Zuwachs an ökonomischer Dispositionskraft ("Reinvermögenszuwachs") als Maßstab der individuellen steuerlichen Leistungsfähigkeit widerspiegeln soll. Entscheidend ist für sie die Auseinandersetzung mit der bis dahin vorherrschenden "Quellentheorie", die Einkommen lediglich als die Summe der bestimmten Einkunftsquellen entspringenden regelmäßig wiederkehrenden Einkünfte betrachtete (Fuisting, 1899, 57). Mit dieser Tradition brach die Reinvermögenszugangstheorie - nicht zuletzt aus Gründen der Gleichmäßigkeit der Besteuerung. So schreibt Schanz (1896, 24): "Wir rechnen also zum Einkommen alle Reinerträge und Nutzungen, geldwerte Leistungen Dritter, alle Geschenke, Erbschaften, Legate, Lotteriegewinne, Versicherungskapitalien, Versicherungsrenten, Kon-

15 Dies insbesondere auch deshalb, weil für die Steuerpflichtigen weder gleiche Präferenzen noch gleiche Wahlmöglichkeiten vorausgesetzt werden können und Änderungen im Steuersystem nie vollkommen unerwartet stattfinden (daher auch die Beibehaltung des Status quo in gewissem Umfang mit capital gains bzw. losses verbunden wäre). Vgl. Feldstein (1976, 77104) sowie die kritischen Stellungnahmen von Hettich (1979, 6f.; 1983, 424f.) und Head (1982, 203-206). Zu Techniken einer Reduzierung von Übergangsproblemen vgl. Goode (1987, 159-169).

16 Eine Berücksichtigung von Einzeldaten, wie sie Hettich (1983, 425f.) vorschwebt, die eine steuerliche Schlechterstellung im Einzelfall verhindern soll, ist - ganz abgesehen vom Informationsproblem - für ein Steuersystem mit für alle Steuerpflichtigen geltenden gleichen Regelungen nicht möglich. 
junkturengewinne jeder Art, wir rechnen ab alle Schuldzinsen und Vermögensverluste. Was erübrigt, steht neu zur Disposition des Empfängers, gehört nicht zu dem bereits vorhandenen Stammvermögen, ...". Haig (1959, 59) definiert ähnlich, wenn auch weniger kasuistisch: "Income is the money value of the net accretion to one's economic power between two points of time", während die Definition von Simons (1938, 50) von der Verwendungsseite ausgeht: "Personal income may be defined as the algebraic sum of (1) the market value of rights exercised in consumption and (2) the change in the value of the store of property rights between the beginning and end of the period in question".

Mit diesen Definitionen sind die wesentlichen Grundlagen für einen Einkommensbegriff gelegt: Vor allem die Universalität des Einkommensbegriffs bei Schanz, die Begriffe "money value", "economic power" und "property rights" bei Haig und Simons sind charakteristisch. Zusammenfassend läßt sich Einkommen daher definieren als einer Person zwischen zwei Zeitpunkten zugeflossene ökonomische Dispositionskraft, gemessen in allgemeinen Kaufkrafteinheiten (Neumark, 1947, 36; Ebnet, 1978, 57).

Als Meßkonzept zur Einkunftsermittlung entspricht der Reinvermögenszugangstheorie am ehesten der Vermögensvergleich. Dieser kann jedoch in Teilbereichen mit durchaus befriedigenden Ergebnissen durch die erheblich einfacheren Verfahren der Einnahmenüberschuß- bzw. Einzahlungsüberschußrechnung angenähert werden ${ }^{17}$. Der von Tipke/ Lang (1991, 200-202) vertretenen Auffassung, die Gewinneinkunftsarten des deutschen Einkommensteuergesetzes basierten auf der Reinvermögenszugangstheorie, die Überschußeinkunftsarten dagegen auf der Quellentheorie, kann daher nicht ohne weiteres gefolgt werden.

Aufbauend auf der gegebenen Einkommensdefinition wird im folgenden auf einige Eigenschaften des Einkommensbegriffs detaillierter eingegangen, sofern sie Problembereiche betreffen, die für die Frage der Besteuerung von Transfereinkommen besonders bedeutsam sind.

17 Vgl. dazu Tipke/Lang (1991, 236-243, 282-286), die in diesem Zusammenhang auch auf Probleme der Durchführung des Vermögensvergleichs verweisen. 


\subsection{Einkommen als objektivierbare Größe}

Mit der oben gegebenen Definition "Zufluß an ökonomischer Dispositionskraft" ist das Einkommen als eine objektivierbare Größe bestimmt, da es allein die Verfügungsmöglichkeiten über prinzipiell marktfähige Güter einschließt (Ebnet, 1978, 55f.; Hackmann, 1983, 666f.).

Demgegenüber setzt das Nutzenkonzept des Einkommens (Ebnet, 1978, 42-49; Hackmann, 1979, 30f.) auf eine sehr viel umfassendere Sichtweise der persönlichen Leistungsfähigkeit, nämlich die tatsächliche Bedürfnisbefriedigung. Dieser Ansatz erlaubt die zusätzliche Berücksichtigung sog. psychischen Einkommens mit entsprechenden individuellen Differenzierungen, z.B. des Freizeitnutzens (Haller, 1981a, 47-50), der Fähigkeit zum Genuß (R.A.Musgrave, 1986, 263), von Arbeitsfreud und -leid (Simons, 1938, 53) ${ }^{18}$ sowie des Versicherungsschutzes ${ }^{19}$, und wäre damit im Hinblick auf das Ziel der Steuergerechtigkeit als überlegen anzusehen 20 . Aufgrund der mangelnden Erfaßbarkeit individueller Nutzen sowie der Unmöglichkeit intersubjektiver Nutzenvergleiche ist er allerdings nicht praktikabel - das steuerliche Einkommen würde zu einer willkürlich vom Steuerpflichtigen oder der Finanzbehörde festgesetzten Größe. Um wenigstens Anhaltspunkte für die individuellen Nutzen zu gewinnen, wäre ein Eindringen in die Privatsphäre der Steuerpflichtigen wahrscheinlich, wie es sich mit einem liberalen Staatsverständnis nur schwerlich vereinbaren ließe (Hackmann, 1979, 30f.; 1983, 665).

Auch wenn hier das Nutzenkonzept des Einkommens abgelehnt und stattdessen vom Wertkonzept des Einkommens (Ebnet, 1978, 55f.) ausgegangen wird, werden Nutzenüberlegungen im Rahmen dieser Arbeit noch herangezogen werden, um Anhaltspunkte bei der Beantwortung bestimmter Fragestellungen zu geben, z.B. $o b$ bestimmte Tatbestände als leistungsfähigkeitsmindernd anzuerkennen sind oder wie die Besteuerung

18 Vgl. zur Steuerreformdiskussion in der Bundesrepublik Wissenschaftlicher Beirat beim Bundesministerium der Finanzen $(1988,3)$.

19 Vgl. dazu besonders die Ausführungen zum Nutzen aus der Vorsorgefunktion der Ersparnis bei Haller (1981a, 57-59).

20 Schneider $(1978,23)$ spricht sehr bildlich vom "siebenten Himmel der Steuergerechtigkeit". 
von Einzelpersonen und Haushalten zueinander geregelt werden soll. Damit wird das hier vertretene Einkommenskonzept nicht in Frage gestellt: Die Höhe evtl. zu berücksichtigender Abzugsbeträge richtet sich nach dem mit objektiven Maßgrößen arbeitenden Wertkonzept des Einkommens ${ }^{21}$.

\subsection{Einkommen als Ist-Einkommen}

Einer Besteuerung nach der Leistungsfähigkeit entspricht es, das tatsächlich erzielte Einkommen, den tatsächlichen Zuwachs an ökonomischer Verfügungsmacht, als Steuerbemessungsgrundlage zu wählen.

Der Besteuerung von Ist-Einkommen (Ex-post-Einkommen) steht die Idee der Besteuerung von Soll-Einkommen (Ex-ante-Einkommen) gegenüber, die aus der Kapitaltheorie entwickelt wurde (Hicks, 1946, 171-181; Mitschke, 1976, 98-105). Unter Effizienzaspekten spricht für das Konzept einer Soll-Einkommensbesteuerung, daß die Höhe der Einkommensteuerschuld dann verhaltensunabhängig ist, Substitutionseffekte also vermieden werden (Arrow, 1973, 260; Hackmann, 1983, 667f.). Auch unter Gerechtigkeitsaspekten ist die Verhaltensunabhängigkeit der Steuerbelastung positiv hervorzuheben, insbesondere wenn berücksichtigt wird, daß eine Ist-Einkommensteuer Arbeitszeit gegenüber Freizeit diskriminiert.

Gleichwohl wird hier die Besteuerung von Soll-Einkommen abgelehnt:

- Nicht alle Einkommenskomponenten sind verhaltensabhängig, was aus Gerechtigkeitserwägungen für die Erfassung der Ex-postEinkommen spricht, wie der Fall der "windfall-profits" deutlich macht 22 .

- Für die Besteuerung auch der verhaltensabhängigen Komponenten spricht ebenfalls der Versicherungseffekt der Einkommensteuer, der

21 Zur Differenzierung zwischen der Definition der Einkünfte in einem Einkommenskonzept und der Verfeinerung dieser Rohgröße durch Berücksichtigung individueller Umstände vgl. auch Pechman $(1968,83)$.

22 Vgl. hierzu die differenzierten Ausführungen von Mitschke (1976, 104f.). 
nur bei einer Besteuerung tatsächlicher Einkommen wirksam wird und durch den Effizienzgewinne erzielbar sind 23 .

- Die Ermittlung von Soll-Einkommen würde erhebliche Probleme aufwerfen und ist daher letztlich nicht praktikabel. Zunächst wären Faktoren zu bestimmen, die für die Höhe des Soll-Einkommens maßgeblich sind (z.B. Qualifikation, Gesundheitszustand). Die Problematik der individuellen Ermittlung solcher Faktoren ist offensichtlich 24 . Tinbergen $(1978,156)$ spricht in diesem Zusammenhang von "psychotechnischen Meßverfahren", ein Ausdruck der jeden liberalen Staatsbürger erschauern läßt. Hinzu kommt die Problematik der Bestimmung des Zeitpunktes (und eventueller Revisionsanlässe) einer solchen individuellen Fähigkeitsveranlagung. Streng genommen und unter Wahrung aller allokativen Vorteile des Konzepts müßte bereits zum Zeitpunkt der Geburt die jährliche Steuerschuld oder gar die Lebenssteuerschuld festgelegt werden; Anpassungen der Steuerschuld im Zeitablauf würden die Diskriminierung von Aktivitäten zur Steigerung des individuellen Einkommenspotentials zur Folge haben 25 .

Daher ist eine Besteuerung von Soll-Einkommen in der Realität weder wünschenswert noch praktikabel - eine Ist-Einkommensbesteuerung ist vorzuziehen. Es muß dabei in Kauf genommen werden, daß ein höherer Verwaltungsaufwand durch die laufende Erfassung der tatsächlichen steuerlich relevanten Einnahmen und Ausgaben erfolgt. Während Einkunftserzielungskosten innerhalb einer Soll-Einkommensteuer bereits pauschal berücksichtigt sind, sind sie bei einer Ist-Einkommensteuer in ihrer tatsächlichen Höhe von den Einnahmen abzuziehen.

23 Vgl. Domar/Musgrave (1959, 493-524), Buchanan (1967, 234-236) sowie Sinn (1988, 65-84).

24 Auf die Gefahren von steuerlichen Anreizen zur Untertreibung persönlicher Fähigkeiten verweist Schneider $(1979,45)$.

25 Zur Unterscheidung zwischen angeborenen und erworbenen Fähigkeiten vgl. Tinbergen $(1978,85 f$.) sowie Schneider $(1979,43)$. Schneider nennt anerzogene Fähigkeiten und Vermögen als Argumente einer Soll-Einkommensteuer, aber auch hier sind bereits steuerlich induzierte Verhaltensänderungen (der Eltern) denkbar. 
Auch jede Form der Typisierung steuerlich relevanter Verhältnisse führt insoweit partiell zu einer Soll-Besteuerung. Gleichwohl können Praktikabilitätsaspekte ein so starkes Gewicht erhalten, daß Typisierungen anzustreben sind 26 . Dies ist insbesondere dann der Fall, wenn die steuerlich relevanten tatsächlichen Verhältnisse nicht überprüfbar sind, so daß an die Stelle einer Typisierung sonst eine Vorteilsmaximierung der Steuerpflichtigen nach ihren jeweiligen individuellen Möglichkeiten treten würde.

\subsection{Einkommen als Entstehung von Eigentum}

Ein konstituierendes Merkmal des hier verwendeten Einkommensbegriffs ist die Entstehung von Eigentum. Das Eigentumsrecht gewährt die uneingeschränkte Verfügungsmöglichkeit über Sachen 27 . Dieser Eigenschaft entspricht eine Beschränkung des Einkommensbegriffs auf Güter, die marktgängig sind, daher auch einen Marktpreis besitzen ${ }^{28}$, und damit eine Erhöhung der aktuellen ökonomischen Dispositionskraft ausdrücken ${ }^{29}$. Es besteht hier eine enge Verbindung zu den bereits behandelten Kriterien "Objektivierbarkeit" und "Ist-Einkommen": Wegen der Ablehnung eines Nutzenkonzepts ist Eigenproduktion im weitesten Sinne, soweit sie nicht genauso gut veräußerbar wäre, ebenso aus dem Einkom-

26 Zum Beispiel ist im Rahmen der Einkunftserzielungskosten eine Pauschalierung bei stark präferenzabhängigen Aufwendungen, die z.T. konsumtiven Charakter haben ("Spesen"), wünschenswert. Vgl. zur allgemeinen Problematik Tipke/Lang (1991, 52f.).

27 Vgl. K.H.Schwab $(1987,110)$. Eine Einschränkung erfährt das Eigentumsrecht nur, soweit Gesetze oder Rechte Dritter dies erfordern.

28 Auf die Frage der Feststellung dieser Marktpreise im Einzelfall braucht an dieser Stelle nicht eingegangen zu werden. Vgl. dazu aber beispielsweise die Ausführungen zur Problematik der Bewertung unrealisierter Wertänderungen bei Ebnet (1978, 149-151).

29 Ausdrücklich eingeschlossen sind daher auch Wertveränderungen im Vermögensbestand. Darüber hinaus kann eine Erhöhung der ökonomischen Dispositionskraft in Sonderfällen auch am Marktpreis von Substitutionsgütern abgelesen werden, um so auch die aktuellen ökonomischen Vorteile nicht veräußerbarer Ausstattungen mit Gütern (z.B. Sozialwohnungen) erfaßbar zu machen. 
mensbegriff ausgeschlossen wie die Bildung von Humankapital 30 und der Erwerb von anderen nicht marktfähigen Rechten. Dabei sind im Rahmen dieser Arbeit insbesondere Ansprüche auf Leistungen aus Versicherungen interessant, deren Realisierung noch vom Eintritt des Versicherungsfalles abhängt. Sofern es sich nicht - wie bei Kapitallebensversicherungen - um Ersparnisbildung unter dem Deckmantel einer Versicherung handelt, sind solche Ansprüche nicht als Vermögenszugang zu werten, da sie aus rechtlichen Gründen (vgl. insb. § 850(3b) ZPO) oder wegen möglicher Gegenauslese (adverse selection) nicht marktfähig sind. Zusammenfassend läßt sich daher Hackmann $(1983,673)$ zitieren: "... der Zeitpunkt des Einkommensanfalls (ist) mithin durch den Zeitpunkt bestimmt, an dem grundsätzlich austausch- bzw. veräußerbare Eigentumstitel und Eigentumsrechte entstanden sind."

\subsection{Einkommen als periodenbezogene Größe}

Um Einkommen überhaupt ermitteln zu können, muß ein Bezug zu einem bestimmten Zeitraum hergestellt werden, da es sich beim Einkommen um eine Stromgröße handelt. Üblicherweise wird das Kalenderjahr als zeitliche Bezugsgröße gewählt. Dies berührt Fragen der Gleichmäßigkeit der Besteuerung, wenn

- die steuerliche ${ }^{31}$ Lebenszeit der Steuerpflichtigen unterschiedlich lang ist und

- im Zeitablauf schwankende Einkommen im Ergebnis stärker belastet werden als gleichförmig fließende Einkommen.

Einer Besteuerung des Lebenseinkommens als Summe der Periodeneinkommen fehlt es an der Festlegung einer objektiven zeitlichen Bezugs-

30 Vgl. Hackmann $(1983,672-675)$ sowie speziell zur Humankapital-Problematik die konträren Ausführungen von Mitschke (1976, 91-93) und Hackmann $(1979,32$, Fn. 47).

31 Unterschiede zur persönlichen Lebenszeit können insbesondere durch den Zeitpunkt des Beginns der Steuerpflicht verursacht werden (Hackmann, 1979, 50-52). 
größe 32 . Eine gleiche Steuerbelastung gleicher Lebenseinkommenssummen bei unterschiedlich langer Lebenszeit wird daher in der Literatur zu Recht abgelehnt ${ }^{33}$. "Da der Wohlstand weitgehend in den jeweiligen Periodeneinkommen zum Ausdruck kommt, diese aber unterschiedlich hoch sind, wenn sich ein gleich hohes Einkommen auf eine unterschiedliche Anzahl von Perioden verteilt, befriedigt diese Lösung nicht" (Albers, 1980, 201). Hier liegt letztlich ein Werturteil zugrunde, das mir aber als Maßstab das einzig vertretbare zu sein scheint ${ }^{34}$.

Dagegen entspricht es der Forderung nach Gleichmäßigkeit der Besteuerung und der Einsicht in die Willkür der Festlegung einer bestimmten Zeitperiode (i.d.R. ein Jahr) als Grundlage der Einkommensbesteuerung, wenn die Sichtweite längerfristig gewählt wird 35 , so daß über einen längeren Zeitraum hinweg - im Extremfall die Lebenszeit gleiche durchschnittliche Periodeneinkommen ungeachtet unterschiedlicher zeitlicher Verläufe gleich behandelt werden.

Zur Verwirklichung dieser Zielsetzung wurden verschiedene Vorschläge einer Lebenseinkommensbesteuerung gemacht, die allerdings

32 Die Wahl des subjektiven Zeitraums "Lebenszeit" entspricht letztlich dem Verzicht auf die Festlegung einer Zeitperiode, da keine Normierung der Stromgröße Einkommen durch Bezugnahme auf eine (objektive) Zeiteinheit stattfindet.

33 Vgl. Birtel (1985, 168f.) und Hackmann (1979, 50f.). Auch die Kritik Schneiders an einer Lebenseinkommensbesteuerung richtet sich auf diesen Punkt und beruht damit auf einer Fehldeutung der hierzu vorgeschlagenen Verfahren. Vgl. dazu Schneider (1978, 51f.) sowie Mitschke (1980, 125f.).

34 Eine entsprechende Regelung findet sich auch bei den personenbezogenen Sozialausgaben, die ebenfalls als bestimmter Betrag pro Periode geleistet werden.

35 In der Verteilungsanalyse entspricht dem die Forderung, Querschnittsbetrachtungen durch die Einbeziehung von individualbezogenen Längsschnittsdaten zu erweitern (Holzmann, 1984, 19-36). 
bisher nur in Ansätzen Eingang in die Steuerpraxis gefunden haben 36 . Im Rahmen dieser Arbeit ist daher auch zu prüfen, ob bestimmte Regelungen der Besteuerung von Transfereinkommen einen Beitrag zur gleichmäßigeren Belastung im Lebensverlauf $z u$ leisten vermögen, sofern von einer Verwirklichung der komplizierteren Lebenseinkommensbesteuerungsvarianten Abstand genommen wird.

Bei den expliziten Verfahren einer Lebenseinkommensbesteuerung wird die Zuordnung der einkommensteuerlichen Bemessungsgrundlage zur Periode der Einkommensentstehung beibehalten, wobei erst die anzuwendende Tarifvorschrift zu einem interperiodischen Ausgleich führt, der (implizit oder explizit) auch Steuererstattungen für frühere Perioden beinhaltet. Ein anderer Weg wird mit der Korrektur der einkommensteuerlichen Bemessungsgrundlage (Reduktion bei Abfluß, Erhöhung bei Zufluß) um bestimmte intertemporale Transaktionen - insbesondere von Versicherungen gegen den zukünftigen Ausfall von Erwerbseinkommen, möglicherweise auch einer allgemeinen Vorsorge in Form von Ersparnis beschritten 37 : Hier wird eine intertemporale (und ggf. auch eine interpersonale) Verschiebung von Teilen der Bemessungsgrundlage zugelassen, die betreffenden Beträge werden - jedenfalls temporär - aus der Steuerpflicht entlassen. Insbesondere durch den damit verbundenen Zinseffekt wird allerdings eine lebenszeitbezogene Gleichmäßigkeit der Besteuerung verfehlt (Hackmann, 1979, 131f., 133-135). Sofern jedoch aus Praktikabilitätsgründen auf eine Anwendung eines die gesamte Lebenszeit der Steuerpflichtigen umfassenden Besteuerungsverfahrens verzichtet wird, bietet eine Sonderbehandlung bestimmter intertemporaler Transaktionen, die vor allem auf längerfristige Probleme (Alterssicherung) abzielt, eine mögliche Ergänzung von kurzfristigeren Durchschnittsbesteuerungsvarianten.

36 Von der theoretischen Konzeption her besonders geeignet erscheinen mir die von Vickrey (1959) und Hackmann (1979, 72f.) entwickelten Verfahren; unter Berücksichtigung der Praktikabilität und Transparenz ist jedoch ein auf einen kürzeren Zeitraum beschränktes Verfahren wie das von der kanadischen Royal Commission on Taxation (1966, Bd.3, 262-269) vorgeschlagene "Block Averaging" vorzuziehen.

37 Vgl. Winterstein $(1965,36)$, Haller $(1973,526)$ sowie Giloy $(1979 a, 135)$. 


\subsection{Zur Gewährleistung der intertemporalen Vergleichbarkeit von Einkommen}

Die Regel, gleiche Einkommen gleich zu besteuern, verlangt in intertemporaler Sicht nach einer Präzisierung. Es stellt sich die Frage, ob bei Vorliegen von rein inflationärem, aber auch darüber hinausgehendem allgemeinem realem Einkommenswachstum immer noch zu verschiedenen Zeitpunkten erzielte gleich hohe Nominaleinkommen als gleich bezeichnet werden können.

Am weitesten verbreitet ist unter Befürwortern der Einkommensteuer die Auffassung, daß die Realeinkommen den Maßstab für einkommensteuerliche Gleichbehandlung abgeben sollten 38 . Festzulegen ist dann allerdings ein Warenkorb, dessen Preisentwicklung für Zwecke der Indexierung 39 maßgeblich sein soll: Um zu vermeiden, daß Preisstrukturänderungen als Inflation ausgewiesen und damit Realwertveränderungen in Teilbereichen durch Indexierung ausgeschaltet werden, ist ein möglichst umfassender Preisindex zu wählen ${ }^{40}$. Der Warenkorb müßte im Idealfall alle Verwendungsmöglichkeiten in einer Volkswirtschaft einschließlich Investitionsgütern und des bereits vorhandenen Güterbestands umfassen, da dies dem aufgrund des Einkommens gegebenen Dispositionspotential entspricht. Für praktische Zwecke bietet sich in erster Linie der Preisindex des Bruttosozialprodukts an ${ }^{41}$; eine ebenfalls weitgehend akzeptierte Lösung dürfte der Preisindex des privaten

38 Vgl. Petersen (1977, 35), Neumark (1977, 304), Meade (1978, 99f.), Ebnet $(1978,92-99)$ sowie Hackmann $(1979,257)$.

39 Diese kann entweder so vorgenommen werden, daß sowohl die Größen der Bemessungsgrundlage als auch des Tarifs und eventueller Freibeträge und Pauschalen inflationiert oder daß die Komponenten der Bemessungsgrundlage auf den Zeitpunkt des Tarif-Basisjahres deflationiert, die sich ergebende Steuerschuld auf aktuelle Werte inflationiert werden (Petersen, 1977, 118-120).

40 Anderer Auffassung sind insbesondere Vertreter der Betriebswirtschaftslehre, die sich für eine Substanzerhaltung in realen Gütern (Indexierung mit Wiederbeschaffungspreisen) statt für eine allgemeine Kaufkrafterhaltung aussprechen (Schneider, 1978, 81-95).

41 Der bereits vorhandene Güterbestand geht hier allerdings nicht ein. 
Verbrauchs $\operatorname{sein}^{42}$. Eine solche Indexierung gewährleistet Gleichmäßigkeit der Besteuerung in intertemporaler Sicht. Unter sonst gleichen Umständen (Steuertarif!) wird so gleich hohe Kaufkraft im Zeitablauf auch steuerlich gleich belastet ${ }^{43}$.

Auf die zwei wichtigsten Alternativen zu einer Realwertindexierung soll im folgenden noch kurz eingegangen werden:

Zum einen wird die Meinung vertreten, zu unterschiedlichen Zeitpunkten angefallene Einkommen sollten unter Verwendung eines Kapitalmarktzinses als Gegenwartswerte vergleichbar gemacht werden 44 . Dies hat zur Folge, daß innerhalb des zugrundegelegten mikroökono-

42 Zur Problematik der Wahl des richtigen Index siehe Denison (1976), Hackmann (1979, 257f.), Ebnet (1978, 98f.) sowie Brümmerhoff (1973/74, 41f.). Angesichts der theoretischen Differenzen ist es beruhigend zu sehen, daß die Werte der in Frage kommenden Indizes sehr nah beieinander liegen (Sachverständigenrat zur Begutachtung der gesamtwirtschaftlichen Entwicklung, 1991, 386f.).

43 Bei über eine Indexierung hinausgehenden diskretionären Tarifanpassungen ergibt sich ein Konflikt mit der Forderung nach Gleichmäßigkeit der Besteuerung. Solche Anpassungen müssen jedoch - sei es wegen veränderter Einschätzung der Leistungsfähigkeitsrelationen, sei es wegen veränderten Staatsausgabenbedarfs - möglich sein; eine Entschärfung des Konflikts bietet sich über eine Zuordnung der Bemessungsgrundlage zum jeweils in der Periode der Einkommenserzielung geltenden Tarif an, wie sie auch in einigen der Lebenseinkommensbesteuerungsverfahren implizit enthalten ist (womit dann nicht die Periodenzuordnung verändert wird, sondern lediglich der Steuersatz bei dem in der jeweiligen Periode gegebenen Tarif).

44 Vgl. Mitschke (1976, 138-144; 1980, 127f.). Letztlich entspricht die damit verbundene Steuerbefreiung von Zinsen weitgehend der Steuerbefreiung der Ersparnis innerhalb einer Persönlichen Allgemeinen Verbrauchsteuer (Pollak, 1987, 63-65). Hiervon zu unterscheiden ist die Verzinsung der Steuerzahlungen, wie sie zuerst von Vickrey vorgeschlagen wurde (Vickrey, 1959; Hackmann, 1979, 169, Fn.10). Die im Rahmen der Steuerreform 1990 beschlossene Verzinsung von Steuernachforderungen und -erstattungen bezieht sich dagegen nur auf die jeweilige Jahressteuerschuld (vgl. § 233a AO; Bundesminister der Finanzen, 1990). 
mischen Wahlhandlungsmodells 45 eine steuerliche Verzerrung der Wahl zwischen Gegenwarts- und Zukunftskonsum vermieden wird. Der verwendete Zins entspricht dann der Grenzrate der Substitution zwischen Gegenwarts- und Zukunftskonsum 46 .

Eine Aussage zur Verbesserung der Allokationseffizienz ließe sich aber nur bei expliziter Berücksichtigung der Entscheidung zwischen Arbeit und Freizeit machen (Musgrave/Musgrave/Kullmer, 1988, 111). Auch entspricht die Besteuerung von Gegenwartswerten des Einkommens der Konzeption einer Soll-Einkommensteuer oder auch einer Persönlichen Allgemeinen Verbrauchsteuer 47 . Nach der in dieser Arbeit vertretenen Auffassung von steuerlicher Leistungsfähigkeit ist eine Besteuerung von Gegenwartswerten des Einkommens daher kritisch zu beurteilen: Sofern tatsächlich Kapitalerträge erwirtschaftet werden, erhöhen diese die Verfügungsmöglichkeiten über Güter (Kaufkraft) und drücken daher steuerliche Leistungsfähigkeit aus; sofern dies nicht der Fall ist, liegt auch keine steuerliche Leistungsfähigkeit vor ${ }^{48}$.

Eine andere Form der Indexierung sieht als Diskontierungsfaktor die Wachstumsrate des Bruttosozialprodukts vor und strebt damit eine Konstanz der volkswirtschaftlichen Steuerquote, also der Aufgabenverteilung zwischen Staat und Privaten, an (Tanzi, 1980, 101-105)49.

45 Andere Sparmotive als der zukünftige Konsum werden nicht berücksichtigt. $\mathrm{Zu}$ möglichen anderen Motiven vgl. Haller (1981a, 57) sowie Andel $(1990,129 f$.$) . Untauglich wird ein derartiges Modell, wenn von einer$ keynesianischen Spar- bzw. Konsumfunktion ausgegangen wird, in der der Zinssatz als Determinante nicht in Erscheinung tritt.

46 Irreführenderweise wird für diesen Sachverhalt oft der Begriff der Zeitpräferenz verwendet. Kritisch dazu Hackmann (1979, 166); zur Begriffsklärung siehe Lehmann $(1975,148-153)$.

47 Zur Äquivalenz der beiden Konzeptionen in dieser Hinsicht vgl. Hackmann (1983, 668f.).

48 Deutlich erkennbar ist die Parallele zur Diskussion um die sog. Doppelbelastungsthese der Ersparnis (Hackmann, 1979, 166; Peffekoven, 1980, 430f.).

49 Dieses Ergebnis stellt sich allerdings exakt nur bei Konstanz der Verteilungsrelationen sowie Übereinstimmung der Veränderungsrate von aggregierter einkommensteuerlicher Bemessungsgrundlage und Bruttosozialprodukt ein. 
Auch wenn hier nicht bestritten wird, daß ein solches Verfahren vor allem ordnungspolitische Vorteile hat, so ist doch die Zielsetzung eine andere als die einer Besteuerung nach der Leistungsfähigkeit. Ein weiteres Eingehen auf diesen Ansatz erübrigt sich daher.

\subsection{Einkommen als personenbezogene Größe}

Einkommen ist nicht objektbezogen, sondern subjektbezogen definiert. Bei der Bemessung der Einkommensteuerschuld können und sollen die persönlichen Umstände der Steuerpflichtigen berücksichtigt werden. Dadurch unterscheidet sich die Einkommensteuer von einer (objektbezogenen) Ertragsteuer (Neumark, 1947, 28f.). Dieser Subjektbezug des Einkommens hat in Verbindung mit dem Grundsatz der Besteuerung nach der Leistungsfähigkeit zur Folge, daß die persönliche Leistungsfähigkeit mindernde Faktoren durch entsprechende Abzüge von der Bemessungsgrundlage zu berücksichtigen sind 50 . Ein prozentualer Abzug von der Steuerschuld ist in diesen Fällen abzulehnen, da eine solche Regelung nicht zu einer symmetrischen Berücksichtigung von leistungsfähigkeitssteigernden und -mindernden Faktoren führen würde 51.

Es ist unvermeidbar, daß eine die persönlichen Umstände der Steuerpflichtigen berücksichtigende Einkommensteuer den ohnehin schon bestehenden Konflikt mit dem Allokationsziel weiter verschärft, weil verstärkt durch einen progressiven Tarif - Faktoreinsätze prozentual unterschiedlich hoch belastet werden und damit gleiche Bruttolöhne bei Unterschieden im privaten Bereich zu differierenden Nettolöhnen führen. Wollte man dieser Kritik an der Einkommensteuer (Brazer, 1980, 227f.) Rechnung tragen, so würde das in letzter Konsequenz eine Rückkehr zur historisch überholt geglaubten (Neumark, 1970, 146f.) Ertragsbesteuerung bedeuten.

50 Vgl. Andel $(1980,376 f$.$) , Peffekoven (1971 / 72,412 \mathrm{f}$.) sowie anderer Ansicht: Schneider (1984) und Biergans/Wasmer (1985, 62f.).

51 Anders verhält es sich bei der steuerlichen Förderung von Ausgaben für bestimmte Zwecke, die aber im Gegensatz zu leistungsfähigkeitsmindernden Ausgaben freie Einkommensverwendung darstellen. Vgl. hierzu auch die späteren Ausführungen in Kapitel III.3.3. 
Unabhängig von der Berücksichtigung der persönlichen Umstände ist die Frage nach der angemessenen Subjekteinheit der Besteuerung ("tax unit") zu behandeln. Ohne eine spätere Diskussion 52 vorwegnehmen zu wollen, läßt sich an dieser Stelle nur feststellen, daß zunächst vom Individuum als maßgeblichem Steuersubjekt ausgegangen werden muß. Zur Erfassung der ökonomischen Aspekte des Beziehungsgeflechts zwischen Individuen ist allerdings auch eine Gruppenbildung von Individuen ("Haushaltsbesteuerung") in Erwägung zu ziehen.

\subsection{Zur Steuerfreiheit des Existenzminimums}

Der Staat darf den Bürgern nicht die zu einem menschenwürdigen Dasein notwendigen Mittel auf dem Weg der Besteuerung nehmen, vielmehr kann eine Steuerpflicht erst da einsetzen, wo finanzielle Mittel für eine Beteiligung an gemeinschaftlichen Aufgaben verfügbar sind 53 . Dies ist ein Gebot der Achtung der Menschenwürde (Art.1 (1) GG) und gilt erst recht, wenn die staatliche Verpflichtung zur Absicherung eines minimalen Lebensstandards über die Sozialhilfe mitbedacht wird54. Diese Ansicht wird in letzter Zeit auch vom Bundesverfassungsgericht (1991a, 85f.) dezidiert vertreten.

Ein Existenzminimum ist allen Bürgern zu gewähren, eine Rücknahme der Steuerbefreiung bei höheren Einkommen nicht vorzusehen 55 . Dies ist - selbst wenn der Einordnung als sozialpolitisch motivierte Steuervergünstigung (Spahn, 1989, 20f.; Müssener, 1991, 30f.) zugestimmt würde - allein schon ein Gebot der Transparenz des Steuertarifs (Andel, 1980, 381).

52 Vgl. Kapitel VI.2.

53 Sofern eine solche Rücksichtnahme auf das Existenzminimum wegen der Erhebungsform bei indirekten Steuern nicht möglich ist, müssen auch deren Belastungen bei den direkten Steuern (und ebenso bei der Bemessung staatlicher Transfers) berücksichtigt werden.

54 So auch Andel (1980, 379-381), Söhn (1988, 162f.) und Pezzer (1989, 223f.); kritisch hierzu: Schmidt $(1988,202)$.

$55 \mathrm{Vgl}$. Tipke/Lang (1991, 210f., 213f.) und Bundesverfassungsgericht (1991a, 86). 
Andererseits ist auch die Gewährung eines mit dem Einkommen ansteigenden Existenzminimumbetrages unter pauschaler Rücksichtnahme auf mit dem Einkommen zunehmende Ansprüche und Verpflichtungen 56 abzulehnen. Die Einräumung eines "standesgemäßen" Existenzminimums widerspricht eindeutig dem Gleichbehandlungsgrundsatz.

Die notwendige Höhe eines (sozio-kulturellen) Existenzminimums läßt sich letztlich nicht objektiv, sondern nur aufgrund eines Werturteils festlegen. Ein Rückgriff auf die Bestimmungen der Sozialhilfe ist naheliegend und zur Herstellung miteinander konsistenter Regelungen im Sozial- und Steuerrecht erforderlich (Lang, 1983, 119; 1987, 25f.). Sowohl die Leistungen der Sozialhilfe als auch die entsprechenden Freibeträge der Einkommensteuer (Grundfreibetrag sowie Ergänzungen nach $\S \S 33,33 \mathrm{~b}$ EStG) sollen dem Umfang nach einem sozio-kulturellen Existenzminimum entsprechen. Im wesentlichen können bei einer solchen Gegenüberstellung der Grundfreibetrag der Einkommensteuer einschließlich der Ergänzung durch Pauschalen nach § 33b EStG der "Hilfe zum Lebensunterhalt", die außergewöhnlichen Belastungen nach $\S 33 \mathrm{EStG}$ der "Hilfe in besonderen Lebenslagen" zugeordnet werden. Allerdings sollte im Steuerveranlagungsverfahren weitestgehend auf Pauschalierungen zurückgegriffen werden, da ein Eingehen auf den konkreten Einzelfall nicht leistbar (Lang, 1988, 202-204), unter Umständen auch aus Gründen der Steuertransparenz und Vermeidung von Steuerverkürzungen nicht wünschenswert ist (Lewis, 1982, 191; Neumark, 1984, 60). Auch kommt es bei den im Einkommensteuerrecht vorzusehenden Beträgen sehr viel weniger als in der Sozialhilfe auf eine Berücksichtigung von akuten Bedarfslagen (Schulte/Trenk-Hinterberger, 1986, 126, 184) an, da der "Kalkulationszeitraum" der Einkommensteuer ohnehin ein Jahr beträgt.

Im einzelnen läßt sich der existentiell notwendige Bedarf in Grundbedarf, Mehrbedarf, außergewöhnlichen Bedarf und Vorsorgebedarf (Lang, 1988, 202-212) zerlegen.

Zum Grundbedarf gehören selbstverständlich alle laufenden Aufwendungen, die bereits im Rahmen der Sozialhilfe pauschal in Form von

56 So Wiese (1928) und Royal Commission on Taxation (1966, Bd.3, 8-12). 
Regelsätzen berücksichtigt werden 57 . Hinzu kommen Aufwendungen für größere Anschaffungen und Instandsetzungen, die die Sozialhilfe außerhalb der Regelsätze berücksichtigt (Schulte/Trenk-Hinterberger, 1986, 181-183). Für das Existenzminimum im Rahmen des Einkommensteuergesetzes können hierfür jahresdurchschnittliche Werte angesetzt werden. Weiter sind die laufenden Aufwendungen für Unterkunft einschl. Heizung zu berücksichtigen, die im Rahmen der Sozialhilfe in Höhe der tatsächlichen Aufwendungen getragen werden 58 . Für einkommensteuerliche Zwecke ist wiederum eine Pauschalierung notwendig. Eine solche Pauschalierung sollte allerdings den örtlichen Unterschieden bei den Mietkosten Rechnung tragen, denn diese sind durchaus erheblich, während bei den übrigen Aufwendungen des Grundbedarfs regionale Unterschiede in den Lebenshaltungskosten kaum ins Gewicht fallen (Angermann, 1989). Eine Regelung beispielsweise in Anlehnung an die Regionalklassen des $\S 8$ WoGG (Mietenstufen) ist meines Erachtens möglich. Schließlich greifen auch private Versicherungsunternehmen in den Bereichen der Kfz-Haftpflicht und der Hausratversicherung zu regionalen Differenzierungen ${ }^{59}$. Selbst bei einer solchen Vorgehensweise bleiben allerdings noch gewisse durch die Pauschalierung bedingte Ungleichbehandlungen bestehen.

Der Mehrbedarf läßt sich als regelmäßig anfallender Bedarf bestimmter Personengruppen (z.B. Behinderte, Alleinerziehende, Alte) beschreiben. Da es sich um einen typisierbaren Bedarf handelt, sind auch hier Pauschalierungen möglich60. Der steuerliche Gleichbehandlungsgrundsatz erfordert sogar eine weitgehende Verwendung von Pauschalie-

57 Vgl. § 22 BSHG, § 1(1) Regelsatzverordnung sowie Schulte/Trenk-Hinterberger (1986, 157-163).

$58 \mathrm{Vgl}$. 3 Regelsatzverordnung sowie Schulte/Trenk-Hinterberger (1986, 165-168).

59 Ein solches Verfahren böte sich auch bei einer eventuellen zukünftigen EG-einheitlichen Einkommensteuer an. Dagegen stößt eine Regionalisierung des Existenzminimums in der Literatur weitgehend auf Ablehnung, vor allem aus Praktikabilitätsgründen (Lang, 1988, 204), aber auch aus regionalpolitischen Erwägungen (Royal Commission on Taxation, 1966, Bd. 3, 21f.).

60 Vgl. §§ 23, 24 BSHG sowie Schulte/Trenk-Hinterberger (1986, 168-172). 
rungen, da bei einer ausschließlichen oder wahlweisen Berücksichtigung tatsächlicher Aufwendungen gleich gelagerte Fälle von Steuerpflichtigen (z.B. mit einer bestimmten Körperbehinderung) steuerlich gerade nicht gleich behandelt würden (Lang, 1988, 552-555). Sofern die Anerkennung eines Mehrbedarfs im Sozialhilferecht ausschließlich mit dem Ziel erfolgt, den Arbeitsanreiz für nicht erwerbstätige, aber erwerbsfähige Sozialhilfeempfänger zu verstärken, ist für einkommensteuerliche Zwecke der allgemeine Grundbedarf entsprechend höher anzusetzen. Die Mehrbedarfszuschläge der Sozialhilfe für Personen über 60 Jahre, Erwerbsunfähige und Erwerbstätige lassen sich in diesem Sinne interpretieren, wenn auch bei den - im Vergleich zu den beiden übrigen Gruppen höheren - Zuschlägen für Erwerbstätige ein Teil zur Abgeltung sonst nicht berücksichtigungsfähiger "Werbungskosten" vorgesehen sein dürfte.

Beim außergewöhnlichen Bedarf handelt es sich um existentiell notwendigen Bedarf, der unregelmäßig und nicht typisierbar anfällt, daher auch in Form eines Grund- bzw. Mehrbedarfs nicht erfaßbar ist. Diese Gruppe ist von der Abgrenzung her mit den außergewöhnlichen Belastungen des § 33 EStG identisch. Für steuerliche Zwecke sollte zunächst versucht werden, durch weitere differenziertere Gruppenbildungen Pauschalierungen im Sinne eines Mehrbedarfs vorzunehmen. Die Fälle außergewöhnlichen Bedarfs werden damit auf eine möglichst geringe Anzahl beschränkt. In Anlehnung an die Vorschriften der Sozialhilfe sind darüber hinaus Verfahrensvorschriften zur Beurteilung der Angemessenheit tatsächlicher Aufwendungen zu entwickeln, die eine steuerliche Berücksichtigung über das Existenzminimum hinausgehender Aufwendungen ausschließen61. Der Ansatz einer zumutbaren Eigenbelastung ist jedoch als systemwidrig abzulehnen (Lang, 1988, 617-619).

Vorsorgebedarf kann, sofern er zur Absicherung gegenwärtiger Risiken dient, im Rahmen des Grundbedarfs erfaßt werden62. Sofern es sich um Altersvorsorge handelt, hängt seine einkommensteuerliche Behand-

61 Vgl. jedoch Lang (1988, 584-597, insb. 595-597).

62 Vgl. aber auch Kapitel V.2.3. 
lung auch unter dem Aspekt einer Sicherung des Existenzminimums vom Gesamtkontext der vorgeschlagenen steuerlichen Behandlung ab63.

\subsection{Volkswirtschaftlicher vs. einkommensteuerlicher Einkommens- begriff}

Zur Bestimmung des einkommensteuerlichen Einkommensbegriffs wird immer wieder auf den volkswirtschaftlichen Einkommensbegriff verwiesen. Damit wird davon ausgegangen, daß die einkommensteuerlich relevanten individuellen Einkommen auf eine Beteiligung an der Erwirtschaftung des Volkseinkommens zurückzuführen sind 64 . Aufgrund unterschiedlicher Zielsetzungen sind jedoch auch unterschiedliche Begriffsabgrenzungen in den beiden Bereichen erforderlich65: Während in der volkswirtschaftlichen Gesamtrechnung die Summe der in einer Periode produzierten Güter (nach Abzug von Vorleistungen) als Zuwachs der in einer Volkswirtschaft verfügbaren Ressourcen erfaßt werden soll, ist für den einkommensteuerlichen Einkommensbegriff die Messung des Zuwachses an individueller Leistungsfähigkeit die Zielgröße. Differenzen treten insbesondere aufgrund von interpersonellen Übertragungen aus laufenden Einkommen oder aus dem Vermögensbestand (aus gesamtwirtschaftlicher Perspektive Doppelzählungen), durch Wertänderungen beim Vermögensbestand und durch individuell nicht zugerechnete Wertschöpfungen auf 66 .

In der Bezugnahme auf den volkswirtschaftlichen Einkommensbegriff liegt eine wichtige Ursache der Ansicht, Transferzahlungen stellten keine

63 Vgl. Kapitel V.1.2.1. Zu den Regelungen im Rahmen der Sozialhilfe, bei denen es auf eine voraussehbare Kostenentlastung des Sozialhilfeträgers ankommt, vgl. Schulte/Trenk-Hinterberger (1986, 172-174).

64 Vgl. Neumark (1947, 41f., 44-47; 1961b, 32f.) und Albers (1957, 80-82).

65 Vgl. Simons (1938, 47, 58) und Ebnet (1978, 71-73). Mit starken Einschränkungen gestehen das auch Neumark $(1961 \mathrm{~b}, 33)$ und Albers $(1957,82) \mathrm{zu}$.

66 Vgl. R.A.Musgrave (1968a, 66) und Pechman (1968, 82). Eine Beschreibung des Zusammenhangs zwischen Bruttosozialprodukt und individuellen steuerlichen Einkommen findet sich bei Musgrave/Musgrave/Kullmer $(1988,145 f$.$) .$ 
steuerliche Leistungsfähigkeit dar und seien daher steuerfrei zu lassen. Diese Ansicht ist geprägt von der Vorstellung, nur produktive Leistungen könnten zur Entstehung von Einkommen führen (während Transferzahlungen gerade keine Faktorentgelte darstellen). Transferzahlungen lassen sich dann allenfalls als Teilhabe am Ergebnis produktiver Leistungen anderer erfassen (Albers, 1957, 80), nicht jedoch als eigenständige Stromgrößen, was nicht nur einen begrifflichen Unterschied bewirkt, sondern jede eigenständige Erfassung von Einkünften, die nicht Ergebnis produktiver Tätigkeit sind, ausschließt. 
Harald Schlee - 978-3-631-75216-6

Downloaded from PubFactory at 01/11/2019 06:59:36AM

via free access 


\section{GRUNDSÄTZE DER EINKOMMENSTEUERLICHEN \\ BEHANDLUNG VON TRANSFERZAHLUNGEN}

\section{Allgemeine Überlegungen}

Transferzahlungen erhöhen die steuerliche Leistungsfähigkeit des Empfängers, sind also voll als Einkommen zu betrachten und dementsprechend zu versteuern. Für den (privaten) Geber dieser Zahlungen handelt es sich um eine Form der Einkommensverwendung, die - sofern keine besonderen Umstände vorliegen - bei der Bemessung der Steuerschuld unbeachtet bleibt ${ }^{1}$.

Insbesondere bei staatlichen Transfers, die - nach Vornahme einer Bedürftigkeitsprüfung - gerade das Existenzminimum des Empfängers abdecken sollen, scheint eine solche Forderung absurd (Schäfer, 1980, 475). Es besteht keine steuerliche Leistungsfähigkeit, die neben der Bestreitung des eigenen Lebensunterhalts auch noch zur Beteiligung an den allgemeinen Staatsaufgaben ausreichen würde. Diesem Umstand wird allerdings innerhalb einer Einkommensteuer, die sowohl ein allgemeines Existenzminimum als auch eventuelle unabweisbare besondere Bedarfe steuerfrei läßt (Berücksichtigung besonderer persönlicher Belastungen) Rechnung getragen. Gegen den Grundsatz einer Einbeziehung aller Einkünfte in die Steuerbemessungsgrundlage läßt sich insoweit also nichts einwenden. Als mögliche Einwände bleiben lediglich die Kosten der verwaltungsmäßigen Erfassung dieser Sozialtransfers sowie die administrativen Probleme der Abstimmung der Existenzminima im Steuerrecht auf besondere Bedarfslagen wie z.B. Fälle von Pflegebedürftigkeit. Diese speziellen Probleme sind später noch zu behandeln.

Auch in einer Besteuerung über das Existenzminimum hinausgehender staatlicher Transferzahlungen könnte - sofern von Finanzaus-

1 Vgl. z.B. Andel (1980, 343f., 354f.), Musgrave/Musgrave/Kullmer (1988, 156f.), Royal Commission on Taxation (1966, Bd.3, 465f.), Simons (1938, 57f.) sowie Deutscher Juristentag (1988, N213). Anderer Auffassung z.B. Graetz $(1980,200)$ und Kirchhof (1988, F24-F26). 
gleichsproblemen zwischen verschiedenen staatlichen Institutionen abgesehen wird - eine letztlich sinnlose Aufblähung von positiven und negativen Transferströmen gesehen werden (Albers, 1967a, 199). Die Auszahlung von Nettobeträgen könnte möglicherweise Verwaltungsaufwand einsparen und die Transparenz des Systems erhöhen, andererseits durch die damit verbundene Einrichtung von Sonderfonds auch genau gegenteilige Wirkungen beinhalten. Eine Steuerfreiheit von Transferzahlungen würde aber ganz offensichtlich einen Verstoß gegen den Grundsatz der Gleichmäßigkeit der Besteuerung bedeuten, da für andere Einkommen in vergleichbarer Höhe eine Steuerpflicht besteht ${ }^{2}$. Auch ein - lediglich gedachter oder tatsächlicher - Steuerabzug vor Auszahlung staatlicher Transfers $^{3}$ wäre keine systematisch befriedigende Lösung: Der Vorteil der Identität von Brutto- und Nettozahlungen würde durch schwer vertretbare Ungleichbehandlungen der Steuerpflichtigen erkauft, deren Ursachen vor allem in dem (direkt und indirekt) progressiven Einkommensteuertarif, möglichen Kumulationen mit anderen (Transfer- und Nicht-Transfer-) Einkünften, der mangelnden Berücksichtigung persönlicher Umstände und den insgesamt damit verbundenen Problemen der Festlegung von Pauschalsteuersätzen zu suchen sind 4 . Lediglich bei summarischer pauschaler Betrachtung führen Steuern zu den gleichen Ergebnissen wie reduzierte Transferzahlungen, eine pauschalierende Betrachtung beinhaltet aber ein Abgehen von der Ermittlung individueller Leistungsfähigkeit. Eine Reduzierung dieser Nachteile bringt die Anwendung des Progressionsvorbehalts nach $\S 32 \mathrm{~b}$ EStG, allerdings nur in bezug auf die Steuerbelastung zusätzlicher Nicht-Transfer-Einkünfte. Hinzu kommt, daß das steuerliche Einkommen wiederum für die Gewäh-

2 Dies ist allerdings dann zu relativieren, wenn die Erzielung eines vom (sonstigen) Einkommen unabhängigen Nettoeffekts das erklärte Ziel staatlicher Transferzahlungen ist.

3 Vgl. dazu die derzeitigen Regelungen zur Berechnung des Arbeitslosengeldes ( $\$ 111 \mathrm{AFG}$ ) sowie zur Rentenanpassung in der gesetzlichen Rentenversicherung ( 868 SGB VI).

4 Vgl. hierzu - allerdings aus stärker sozialpolitischer Sicht - Schäfer (1980, 464-469). Zur Bedeutung von Kumulationen bei staatlichen Transferzahlungen vgl. Transfer-Enquête-Kommission (1981, 104-123) sowie Weyers/ Oberhauser (1984). 
rung staatlicher Transfers herangezogen wird, so daß auf diese Weise die Möglichkeit weiterer Begünstigungen durch die einkommensteuerliche Behandlung von Transfereinkünften gegenüber anderen Einkünften besteht (Andel, 1980, 344; 1990, 295). Daß das Niveau staatlicher Transferzahlungen bei Einführung einer Steuerpflicht unter Umständen angehoben werden muß, um gegebene sozialpolitische Zielsetzungen zu erreichen (Andel, 1980, 344; Schäfer, 1980, 477), ist richtig. In diesem Falle wären auch Finanzierungsfragen zu klären, allerdings ohne eine Zweckbindung von Steuermehreinnahmen zu etablieren 5 . Auch hier handelt es sich letztlich nicht um ein überzeugendes Argument gegen den Grundsatz einer Steuerpflicht von Transfereinkommen. Schließlich subventioniert eine Steuerfreiheit von Transferzahlungen letztendlich die zur Transferzahlung verpflichtete Institution. Sofern es sich um direkte staatliche Transferzahlungen handelt, mag dies gerechtfertigt sein; anderenfalls wird die Gesamtheit der Steuerzahler mit Aufgaben belastet, die von anderen privaten oder öffentlich-rechtlichen Institutionen $\mathrm{zu}$ finanzieren sind6.

Eine Besteuerung beim (privaten) Transfergeber wird zum Teil mit der Begründung abgelehnt, durch die Transferzahlung werde dessen steuerliche Leistungsfähigkeit reduziert (Andrews, 1972/73, 362-366). Dies mag zwar für besondere Situationen (z.B. bei Vorliegen gesetzlichen Zwangs) zu diskutieren sein; ohne daß solche besonderen Umstände vorliegen, handelt es sich jedoch auch bei Transfers lediglich um eine Form prinzipiell steuerpflichtiger Einkommensverwendung7. Eine Steuerfreiheit von Transfers ist auch nicht gerechtfertigt, um Anreize zu

5 Eine Bindung aufgrund einer Transferbesteuerung erzielter Steuermehreinnahmen an die Finanzierung höherer staatlicher Bruttotransferzahlungen erscheint allenfalls für eine Übergangsfrist vertretbar.

6 Dies wird in Haftpflichtfällen, die beispielsweise im Rahmen von Privathaftpflichtversicherungen oder der gesetzlichen Unfallversicherung abgewickelt werden, besonders deutlich, wo durch die Steuerfreiheit von Einkommensersatzleistungen nicht die vollen Kosten verursachergerecht angelastet werden.

7 Hinzu kommt, daß dann unbeschränkte Möglichkeiten einer Steuerarbitrage eröffnet würden. 
privaten Transferzahlungen zu geben ${ }^{8}$. Sollte es allgemeine Gründe für die Förderungswürdigkeit von Transfers geben, wäre nicht ein Abzug von der Bemessungsgrundlage des Transfergebers (der wegen der Tarifprogression zu unterschiedlichen "Prämien" je nach Einkommenshöhe führen würde), sondern ein für alle Steuerzahler gleich hoher Abzug von der Steuerschuld einzuräumen (Peffekoven, 1971/72, 414-417; Paqué, 1986, 344-349). Solche allgemeinen Gründe liegen jedoch - jedenfalls innerhalb einer Einkommensteuer ${ }^{9}$ - nicht vor; für bestimmte Transferarten vorgetragene spezielle Begründungen für eine völlige Steuerfreiheit bzw. eine staatliche Prämierung geleisteter Transfers werden später noch zu behandeln sein 10 .

Als Ergebnis läßt sich daher festhalten, daß sowohl staatliche als auch private Transferzahlungen die Leistungsfähigkeit ihrer Empfänger erhöhen, während die Leistungsfähigkeit des Transfergebers durch diese Form der Einkommensverwendung nicht berührt sein muß. Grundsätzlich ist daher eine Steuerpflicht beim Empfänger, jedoch keine steuerliche Abzugsfähigkeit beim Leistenden, vorzusehen. Abweichungen von diesem Grundsatz sind insbesondere bei gesetzlich vorgeschriebenen Transfers sowie bei staatlichen Förderprämien angebracht. Auf diese Fälle wird noch einzugehen sein.

8 Für eine Beschreibung des hier zugrundeliegenden Modellrahmens, der auch gruppenspezifische Förderprämien umfassen kann, vgl. Clotfelder (1985, 280-285) und Paqué (1986, 158-241).

9 Innerhalb einer Persönlichen Allgemeinen Verbrauchsteuer wären Transferzahlungen kein Konsum im üblichen Sinne, allerdings auch keine Ersparnis, daher wäre evtl. eine Steuerfreiheit beim Transfergeber zu vertreten (so Naust, 1991/92, 503). Wenn jedoch berücksichtigt wird, daß die Persönliche Allgemeine Verbrauchsteuer eine Besteuerung des - allerdings pauschalierten - Nutzens zum Ziel hat (Schneider, 1971, 364-372; Ebnet, 1978, 47f.), ist auch in einem solchen System eine Steuerfreiheit nicht allgemein gerechtfertigt. Hinzu kommen die gesellschaftspolitisch fatalen Wirkungen, wenn auf eigener Leistung beruhende Einkommen besteuert werden, Transfereinkommen, insb. auch die Übertragung großer Vermögensmassen, aber unbesteuert bleiben.

$10 \mathrm{Vgl}$. zu allgemeinen Aussagen hierzu Kapitel III.3.3, zu entsprechenden Argumenten bei bestimmten Arten von Transfers die Kapitel IV - VI. 


\section{Zur Eignung des Korrespondenzprinzips als generelle Regelung}

\subsection{Definition}

Das Korrespondenzprinzip findet sich in der Literatur in verschiedenen Interpretationen. Dabei sind Formulierungen wie "alle Einkommen (sollten) einmal besteuert werden" (Albers, 1977, 906, 910) allgemein konsensfähig, beinhalten aber ohne eine Bestimmung dessen, was unter Einkommen verstanden werden soll, auch keine neuen Einsichten. Daher wird hier zunächst auf die sehr prägnante Definition von Littmann (1983, 431) zurückgegriffen: "Es (das Korrespondenzprinzip, H.S.) verlangt, daß jedes Einkommen einmal - jedoch nur einmal - steuerlich erfaßt wird, daß also bei jeder Einkommensübertragung entweder der Leistende oder der Empfänger einkommensteuerpflichtig sein muß." Littmann konkretisiert diese Aussage noch für den Fall intertemporaler Übertragungen: "Unter steuerlichen Gesichtspunkten ist es unbeachtlich, ob bei intertemporalen Übertragungen Leistender und Empfänger identisch sind oder nicht ... Kann der Leistende - gleichgültig, ob er später Empfänger der Übertragungen sein wird oder nicht - seine Aufwendungen steuerlich geltend machen, dann muß konsequent der Empfänger die Übertragung versteuern, und zwar in dem Veranlagungszeitraum, in dem ihm das abgeleitete Einkommen zufließt. Umgekehrt ist zu entscheiden, wenn der Leistende seine Aufwendungen zur Einkommensübertragung versteuern muß." (Littmann, 1983, 431)11.

Mit dem Korrespondenzprinzip wird also die für die Einkommensteuer typische subjektbezogene Betrachtung mit einer objektbezogenen verbunden oder ganz durch eine solche ersetzt.

\subsection{Interpersonales Korrespondenzprinzip}

$\mathrm{Da} \beta$ bei Einkommensübertragungen Leistungen, die beim Leistenden steuerpflichtig sind, beim Empfänger steuerfrei sein sollten und

11 Die Kompliziertheit der Ausführungen erklärt sich dadurch, daß es nicht um den einfachen Fall der Ersparnis geht, sondern um Versicherungen, die einen Risikoausgleich zwischen den Versicherten vornehmen. 
umgekehrt, steht in Widerspruch zu den Grundsätzen der Einkommensteuer12: Zum einen ist nicht einzusehen, warum nicht sowohl beim Leistenden, für den Transferzahlungen Einkommensverwendung darstellen, als auch beim Empfänger, für den die entsprechenden Beträge das verfügbare Einkommen erhöhen, steuerliche Leistungsfähigkeit, und das heißt Steuerpflicht, bestehen soll. Zum anderen kann es nicht beliebig sein, ob eine Transferzahlung beim Leistenden oder beim Empfänger besteuert wird, da es auf die individuelle Erfassung der steuerlichen Leistungsfähigkeit ankommt, und nicht etwa darauf, den entsprechenden Betrag bei irgendeiner Person überhaupt einmal zu besteuern. Allein schon der Umstand, daß die individuellen marginalen Steuersätze differieren, ist ein zusätzliches unmittelbar einleuchtendes Argument. Typisch für die Forderung nach interpersonaler Korrespondenz ist die Bezugnahme auf aggregierte Einkommensgrößen der Volkswirtschaftlichen Gesamtrechnung; eine solche Bezugnahme ist der Einkommensteuer aber fremd 13. Auch aus finanzwissenschaftlicher Sicht ist daher Söhn (1985, 406) zuzustimmen: "... nur eine Besteuerung nach der individuellen wirtschaftlichen Leistungsfähigkeit des Gebers und des Empfängers ist (verfassungs)rechtlich zulässig und gewährleistet die individuelle Gleichheit der Besteuerung."

\subsection{Intertemporales Korrespondenzprinzip}

Ein Prinzip, daß bei intertemporalen Einkommensübertragungen entweder der Leistende oder der Empfänger steuerpflichtig sein sollte, stellt ebenfalls auf eine objektbezogene Sicht ab, nach der jede Einkommensübertragung lediglich einmal $\mathrm{zu}$ versteuern ist. Institutionell wird das intertemporale Korrespondenzprinzip insbesondere auf Beiträge an und Leistungen von Altersrentenversicherungen bezogen.

$12 \mathrm{Vgl}$. für eine kritische Auseinandersetzung mit dem interpersonalen Korrespondenzprinzip Tipke $(1980,8)$, Söhn $(1985,405 f$.) sowie Lang (1988, 84).

13 Vgl. Simons $(1938,57 f$.$) sowie Kapitel II.2.9.$ 
Als Bezugspunkt dient die einkommensteuerliche Behandlung der Ersparnis: Ersparnis ist als Einkommensverwendung aus versteuertem Einkommen zu bilden, der reine Kapitalrückfluß ist (daher) steuerfrei zu lassen, lediglich Zinsen stellen einen Vermögenszuwachs dar und sind als solcher der Einkommensteuer zu unterwerfen. Eine objektbezogene Sicht, die Trennung in Vermögensbestand und Vermögenszuwachs, ist angemessen, da es um Vorgänge bei ein und demselben Steuerpflichtigen geht.

Das intertemporale Korrespondenzprinzip behauptet nun eine Äquivalenz des eben beschriebenen "vorgelagerten Besteuerungsverfahrens" mit einem "nachgelagerten Besteuerungsverfahren"14, bei dem die Ersparnisbildung steuerfrei bleibt, dafür jedoch die Auflösung der Vermögensbestände zu versteuern ist. Diese Äquivalenz bezüglich des Zeitpunkts des Steuerzugriffs ist für Spar- und Entsparvorgänge zutreffend, jedenfalls sofern vom Nominalprinzip ausgegangen wird und Zinsen außer Betracht bleiben.

Dagegen ist eine Übertragung dieses Äquivalenzgedankens auf Altersrentenversicherungen, bei denen eine Identität von Beitragszahlern und Leistungsempfängern gerade nicht gegeben ist, nicht zulässig. Es liegt im Wesen einer solchen Versicherung, Umverteilungen unter den Mitgliedern vorzunehmen, also intertemporale und interpersonale Transaktionen zu verknüpfen. Eine Einkommensteuer, die auf den Vermögenszuwachs abstellt, hat diese Vorgänge individuell zu berücksichtigen. Eine objektbezogene Sicht wie in der oben angeführten Definition von Littmann, nach der auch dann ein (steuerlich unerheblicher) Kapitalrückfluß konstatiert wird, wenn sich die individuellen "Vermögensverhältnisse" aufgrund von Umverteilungen stark verschoben haben, ist steuersystematisch nicht zu vertreten. Auch ein Rückgriff auf Erwartungswerte, demzufolge versicherungsimmanente Umverteilungsvorgänge steuerlich unbeachtlich wären, lediglich Abweichungen vom Versicherungsprinzip als Umverteilung steuerlich erfaßt würden (Albers, 1980, 200), ist mit einer IstEinkommensteuer nicht vereinbar ${ }^{15}$.

14 Zur Verwendung der Begriffe vgl. Wissenschaftlicher Beirat beim Bundesministerium der Finanzen $(1986,519)$ und Henke $(1987,30)$.

15 Vgl. Kapitel II.2.3. 
Als steuersystematisch korrekte Lösung bleibt nur eine individuelle Erfassung aller Umverteilungsgewinne und -verluste sowie der Zinskomponente (Andel, 1970, 332f., 339; Pollak, 1979, 264-266), womit die Vermischung von intertemporaler und interpersonaler Komponente aufgehoben wird. Bei einer entsprechenden individuellen steuerlichen Lösung 16 geht jedoch auch die Einfachheit des Besteuerungsverfahrens, wie sie die oben geschilderte Definition ermöglicht, wonach entweder Leistungen oder Beiträge zu versteuern sind, verloren.

Die Beliebtheit der Verwendung des Korrespondenzprinzips läßt sich aber gerade auf die damit bei Verwendung von Definitionen wie der oben geschilderten mögliche Vernachlässigung der Prüfung individueller steuerlicher Leistungsfähigkeit zurückführen. Das zeigt ein Blick in die Literatur, wo unter Berufung auf das "intertemporale Korrespondenzprinzip" zum Teil Zinsen und Umverteilungsgewinne und -verluste völlig außer Betracht gelassen werden (Steuerreformkommission, 1971, 123f., Rz.239), lediglich versicherungsfremde Umverteilungen steuerlich berücksichtigt werden (Albers, 1980, 200)17 oder die Steuerfreiheit von Zinsen ausdrücklich erwogen wird (Albers, 1980, 200; Wissenschaftlicher Beirat beim BMF, 1986, 520).

Auch die in Teilen der Literatur anzutreffende, den Vertretern eines "intertemporalen Korrespondenzprinzips" widersprechende, Auffassung, die Steuerfreiheit von Beiträgen an Altersrentenversicherungen sei als gewollte Steuervergünstigung und daher als Steuerverzicht des Staates zu interpretieren, ein späteres Nachholen der Steuerpflicht sei nicht zulässig18, ist letztlich nur wegen der mangelnden Verankerung des "intertemporalen Korrespondenzprinzips" in einer Besteuerung nach der individuellen Leistungsfähigkeit (und deren Konkretisierung im Einkommensteuergesetz) möglich. Die daraus resultierenden Folgerungen widersprechen zwar dem Ziel einer möglichst lückenlosen Erfassung steuer-

16 Vgl. hierzu Kapitel V.1.3.4.1(2).

17 Nach Abwägung anderer Möglichkeiten auch Andel $(1970,333)$ sowie Brümmerhoff $(1979,221 f$.).

18 Vgl. Birk (1986a, 26f.), Schmähl (1986, 112) und Zitzelsberger (1984, 472). 
licher Leistungsfähigkeit (im Sinne einer Comprehensive Tax Base), die Logik des geltenden Einkommensteuerrechts spricht aber durchaus für eine solche Argumentation.

Um zu einer steuersystematisch befriedigenden Lösung zu gelangen, ist eine Rückbesinnung auf die Reinvermögenszugangstheorie des Einkommens notwendig. Die stark von Pragmatismus geprägten Formulierungen eines "intertemporalen Korrespondenzprinzips" dienen eher einer Vernebelung der steuerlich relevanten Sachverhalte. Die Isolierung der rein intertemporalen Komponente des Korrespondenzprinzips unter Ausschaltung interpersonaler Faktoren läßt sich ebenso gut mit den Begriffen Steueraufschub bzw. Steuerstundung umschreiben, die erheblich klarer sind.

\section{3. Überlegungen zu Sonderaspekten}

\subsection{Gefahrengemeinschaften}

Gefahrengemeinschaften sind Transfergemeinschaften in dem Sinne, daß ihre Mitglieder sich gegenseitig verpflichten, in einem genau festgelegten Risikofall Transfers an das betroffene Mitglied zu leisten. Möller (1950, 29) spricht in diesem Zusammenhang von "Rechtsansprüchen auf gegenseitige Bedarfsdeckung". Bei Gefahrengemeinschaften handelt es sich um die Grundform der Versicherung (Mahr, 1951, 76). Bei den heute anzutreffenden Versicherungen finden allerdings in der Regel keine direkten Transferzahlungen der Mitglieder untereinander statt, sondern ein Unternehmen mit Gewinnerzielungsabsicht übernimmt die Ex-anteKalkulation von Beiträgen und Risiken. In der Versicherungsbetriebslehre stehen denn auch die Managementaufgaben des Versicherers im Vordergrund. Daher distanziert sie sich vom Begriff der Gefahrengemeinschaft, der unternehmerischen Kalkülen nicht die angemessene Bedeutung einräumt ${ }^{19}$. Um den Charakter von Beiträgen und Leistungen von Versiche-

19 Müller (1988) spricht aus diesem Grund vom "Gefahrengemeinschaftsmythos". 
rungsunternehmen im hier interessierenden Zusammenhang herauszuarbeiten, ist aber gerade das Gefahrengemeinschaftsmodell gut geeignet.

Zum Fall der direkten privaten Transfers besteht allerdings ein wesentlicher Unterschied: Im Falle der Gefahrengemeinschaft werden Transfers vor allem auch mit der Absicht der Verstetigung des eigenen Einkommens geleistet 20 . Aus Ex-ante-Sicht liegt - abgesehen von eventuellen Verwaltungskosten und einer Risikoprämie des Versicherungsunternehmens - Äquivalenz vor, d.h. die Erwartungswerte von Beiträgen und Leistungen entsprechen einander. Mit dem Eingehen einer Zahlungsverpflichtung in der einfachen Gefahrengemeinschaft bzw. mit der Leistung von Beiträgen an ein Versicherungsunternehmen erwirbt der Versicherungsnehmer für den Risikofall selbst einen entsprechenden Leistungsanspruch. Im Zeitpunkt der Beitragszahlung wird damit i.d.R. kein Vermögen gebildet, sondern lediglich ein Anspruch erworben, dessen Realisierung vom Eintritt einer Bedingung ("Risikofall") abhängt.

Auch wenn der Abschluß von Versicherungen im eigenen Interesse erfolgt und damit sowohl bei einer nutzenorientierten Sicht, bei der entscheidend ist, daß der Versicherungsschutz offensichtlich als mindestens gleichwerte Gegenleistung betrachtet wird, als auch aus der Sicht eines Ex-ante-Einkommens, bei der die im Risikofall zu erwartende Versicherungsleistung in Rechnung zu stellen ist, keine Minderung der Leistungsfähigkeit festgestellt werden kann, so kommt es doch aus der Sicht der Reinvermögenszugangstheorie allein auf den (ex post) festzustellenden Vermögenszuwachs an. Daher ist es entscheidend, ob die Versicherungsbeiträge zu einer Vermögensbildung führen, was - sofern zukünftige Risiken abgesichert werden - von der Marktfähigkeit und dem Marktwert der erworbenen Ansprüche (i.S. eines Veräußerungswerts für

20 Versicherungen im Konsumbereich werden hier nicht weiter betrachtet, da sie einkommensteuerlich irrelevant sind, d.h. weder Beiträge noch Leistungen Steuerabzugsfähigkeit oder Steuerpflicht begründen (sofern keine Überversicherung vorliegt, wird lediglich die Erhaltung eines vorhandenen Bestandes abgesichert). Ebenfalls wird zunächst von Versicherungen zugunsten Dritter abgesehen. 
den Versicherungsnehmer) abhängt ${ }^{21}$. Ist ein solcher Marktwert festzustellen, so handelt es sich bei den Beiträgen zur Sicherung zukünftigen Einkommens offensichtlich in dem Maße um eine Form der Ersparnis, in dem sich die Beiträge im Marktwert niederschlagen. Sofern dies nicht der Fall ist, handelt es sich zwar um private Transfers, die jedoch aus einkommensteuerlicher Sicht als Einkunftserzielungskosten für die Erzielung zukünftiger Einnahmen zu klassifizieren sind, da es sich um "Aufwendungen zur Erwerbung, Sicherung und Erhaltung der Einnahmen" ( $§ 9(1)$ EStG) handelt. Nach dem sogenannten einkommensteuerlichen Nettoprinzip sind sie demnach von der steuerlichen Bemessungsgrundlage abzuziehen 22 . Versicherungsleistungen sind voll zu versteuern, da sie einen Vermögenszugang und damit eine Erhöhung der steuerlichen Leistungsfähigkeit darstellen.

Auch wenn eine Einordnung von Beiträgen zur Zukunftssicherung als Ersparnis nach den eben genannten Argumenten i.d.R. abzulehnen ist, soll auch diese Sichtweise im weiteren Verlauf der Arbeit weiterverfolgt werden, da sie in der Literatur sehr verbreitet ist 23 . Gleichzeitig kann die einkommensteuerliche Behandlung der Ersparnis als Referenzfall dienen, wenn es um die Beschreibung der Auswirkungen bestimmter steuerlicher Regelungen auf die zu erwartende relative Vorteilhaftigkeit (Rendite) von Versicherungen in der (Ex-ante-)Einschätzung der Steuerpflichtigen bei

21 Vgl. dazu § 176 VVG, § 850(3b) ZPO, Klanberg (1984, 418f.). Auch § 4 und $\S 12(4)$ BewG sowie Rössler/Troll $(1989,98,156)$ sind in diesem Zusammenhang aufschlußreich.

22 Die Meinungen über die Modalitäten eines Werbungskostenabzugs gehen in der Literatur allerdings auseinander. So strebt Littmann $(1983,485)$ eine Zuordnung von Werbungskosten oder Betriebsausgaben - wohl wegen gegenwärtigen Vorsorgebedarfs - zu den im Alter wegfallenden Einkünften an; Söhn $(1985,404)$ findet mit der Zuordnung vorab entstandener Werbungskosten zu § 22 EStG zwar eine systematisch befriedigendere Lösung, greift damit aber bei Beibehaltung der Regelungen des $\S 22 \mathrm{Nr}$.1 EStG zu kurz. Vgl. zur Anerkennung als Werbungskosten im Rahmen des derzeitigen Einkommensteuergesetzes Bundesfinanzhof (1986), Geiger (1987), Giloy (1979b) sowie Birk (1986b, 108-111).

23 Vgl. das Kapitel "Intertemporales Korrespondenzprinzip". 
einer unterstellten Wahlentscheidung zwischen Versicherung und traditioneller Ersparnis geht.

\subsection{Zwangsaspekte}

Zwangstransfers sind insbesondere in Form von Steuern, Unterhaltsverpflichtungen und Zwangsversicherungen anzutreffen. Sofern sie nicht mit einer freiwilligen Entscheidung zur konsumtiven Einkommensverwendung verknüpft sind 24 und die Leistungsfähigkeit des Transferleistenden reduzieren, sollten sie zu einem Abzug von der Bemessungsgrundlage führen.

Von Zwangstransfers soll hier nur dann gesprochen werden, wenn der Transferleistende direkt durch gesetzliche Bestimmungen zur Transferzahlung verpflichtet wird; Selbstverpflichtungen von Steuerpflichtigen, die Ausdruck ihrer persönlichen Entscheidungsfreiheit sind, werden damit aus der Betrachtung ausgeschlossen 25 . Im Rahmen der hier für steuerliche Zwecke notwendigen verallgemeinernden Betrachtungsweise ist eine weitere Differenzierung von auf gesetzlichem Zwang beruhenden Transfers danach, ob ihre Höhe im Einzelfall exakt nachvollziehbar ist (z.B. Steuern, Versicherungsbeiträge) oder nur pauschalierend vermutet werden kann (z.B. bei innerfamiliären Unterhaltszahlungen), nicht sinnvoll. Vielmehr wird davon ausgegangen, daß die rechtlichen Bestimmungen zur Geltung kommen, auch wenn eine Überprüfung im Einzelfall nicht möglich ist.

Steuern, die in Zusammenhang mit der Einkunftserzielung stehen, insbesondere solche, die ebenfalls auf die Bemessungsgrundlage Einkom-

24 Dies ist beispielsweise bei der Kraftfahrzeug-Haftpflichtversicherung als Zwangsversicherung der Fall. Der Konsumbereich ist für die Einkommensteuer grundsätzlich unerheblich; mit Investitionen verbundene Zwangstransfers sind als Einkunftserzielungskosten ohnehin zu berücksichtigen.

25 Wie noch zu sehen sein wird, muß hier allerdings im Einzelfall aufgrund eines Werturteils entschieden werden, wie weit in der Ursachenkette zurückgegangen werden sollte, um diese Feststellung zu treffen (vgl. Jakob/Jüptner, 1983, 210-214). 
men zugreifen, sollten von der Bemessungsgrundlage der Einkommensteuer (des Zentralstaates) abgezogen werden können. Dies gilt z.B. für von untergeordneten Gebietskörperschaften erhobene Einkommensteuern $^{26}$. Zu denken ist aber auch an Zwangsbeiträge zu Sozialversicherungen, sofern bereits aus Ex-ante-Sicht Umverteilungselemente enthalten sind, z.B. derart, daß die Bemessungsgrundlage der Beiträge das Einkommen bildet, gleichzeitig jedoch - im einfachsten Fall - die Leistungen beitrags- (und damit einkommens-) unabhängig gewährt werden 27 . Die Differenz zwischen tatsächlichem Beitrag und Durchschnittsbeitrag ist dann als Steuer zu betrachten 28 .

Abbildung 1:

Steuercharakter von Sozialversicherungsbeiträgen

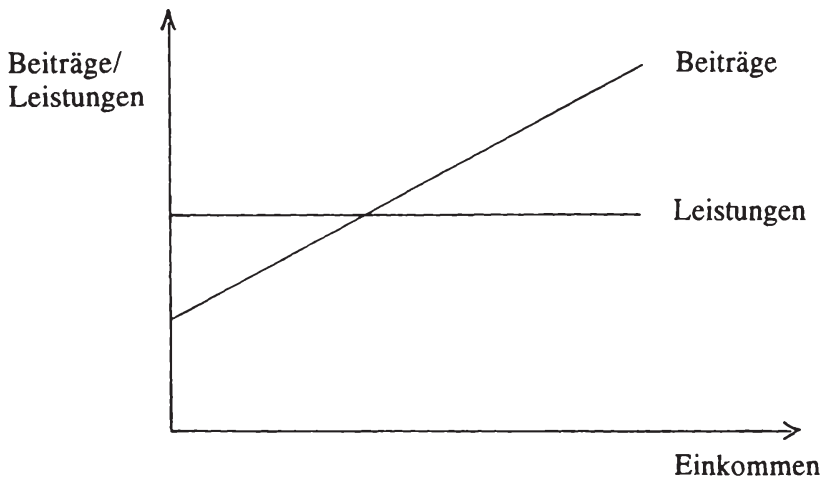

Quelle: Hansmeyer $(1963,109)$.

Die Behandlung von Unterhaltsverpflichtungen ist unter dem Zwangsaspekt problematischer: Von Zwang kann allgemein nicht bereits dann gesprochen werden, wenn eine freiwillige Selbstverpflichtung des Steuer-

26 Vgl. Andel $(1980,373)$. Strittig ist die Einordnung der Vermögensteuer als selbständige Leistungsfähigkeitssteuer oder als Teil der Einkunftserzielungskosten.

27 Diese Beschreibung trifft weitgehend auf die gesetzliche Krankenversicherung zu.

28 Vgl. hierzu Transfer-Enquête-Kommission (1981, 260, Rz.456) und Peter$\operatorname{sen}(1982,389 f$. $)$. 
pflichtigen in Form eines Vertrags vorliegt, sondern nur dann, wenn auch der Vertragsabschluß selbst zwangsläufig war. Daraus folgern Jakob/ Jüptner $(1983,213)$ vollkommen richtig: "Weder die Kosten für die Ausbildung eines Kindes noch der Unterhalt an die Frau (oder den Mann, H.S.) ... wären abzugsfähig, wenn man auf die freiwillige Entscheidung zur Eheschließung oder für Nachwuchs abstellte." Offensichtlich liegt bei der einkommensteuerlichen Berücksichtigung von Unterhaltsverpflichtungen - jedenfalls solange ihre Höhe im gesetzlichen Rahmen bleibt - ein Werturteil zugrunde (Jakob/Jüptner, 1983, 213), das unter Berufung auf übergeordnete Zielsetzungen (insb. auch Art. $6 \mathrm{GG}$ ) die besondere Qualität innerfamiliärer Transfers hervorhebt und die freiwillige Entscheidung der Steuerpflichtigen ${ }^{29}$ für die Ehe bzw. für Kinder hinter den - erst daraus resultierenden - Zwang zum laufenden Unterhalt zurückstellt ${ }^{30}$.

Die Rolle, die der Familie und insbesondere dem Aufziehen von Kindern in unserer Gesellschaft zukommt, rechtfertigt eine steuerliche Berücksichtigung der Unterhaltsverpflichtungen. Eine Anerkennung wird auch dadurch gestützt, daß für diesen Bereich durchweg gesetzliche Bestimmungen vorgegeben werden, deren Einhaltung zwar nicht im Einzelfall überwacht, aber spätestens im Konfliktfall zwischen den beteiligten Personen relevant wird. Diese zivilrechtlichen Vorschriften sollten eine Entsprechung im Steuerrecht finden. Schließlich ist es schwerlich mit der heutigen Auffassung von Menschenwürde zu vereinbaren, Ausgaben für den zwangsläufigen Unterhalt von Familienangehörigen als Konsum einzustufen. Daher ist es meiner Ansicht nach geboten, solche zwangsläufigen Unterhaltsleistungen als Minderung der Leistungsfähigkeit einkommensteuerlich zu berücksichtigen.

29 Eine Ausnahme bilden hier Unterhaltszahlungen von Kindern an ihre Eltern, die kaum als Folge einer freiwilligen Entscheidung der Kinder eingestuft werden können.

30 Sonst würde die Entscheidung genauso wie z.B. die Aufnahme eines Konsumentenkredits dem Konsumbereich zugeordnet: Bei "gleicher Sachlage" (Zwang zur Zinszahlung und Tilgung) ist dessen einkommensteuerliche Unbeachtlichkeit recht unstrittig. 
Innerfamiliären Unterhaltsverpflichtungen gleichzustellen sind Transferleistungen, die der Sicherung des Existenzminimums anderer Personen, die nicht unbedingt in einem Verwandtschaftsverhältnis zum Leistenden stehen müssen, dienen. Die Anerkennung des Subsidiaritätsprinzips, nach dem eine Aufgabenwahrnehmung durch möglichst kleine gesellschaftliche Einheiten erfolgen sollte, gebietet es, die entsprechenden Leistungen beim Transfergeber als leistungsfähigkeitsmindernd anzuerkennen, da anderenfalls die staatliche Sozialhilfe als Leistungsträger einspringen müßte. Von praktischer Bedeutung können solche - womöglich auf privatrechtlichen Verträgen beruhende - Leistungen insbesondere in nichtehelichen Lebensgemeinschaften sein, in denen sie derzeit zum Teil einseitig sozialrechtlich unterstellt werden (Brühl, 1989, 154-157).

Zwangsbeiträge an Versicherungen sind insofern anders geartet, als sie regelmäßig einen Anspruch auf Gegenleistung nach sich ziehen. Es ist daher voreilig, allein aus dem Vorliegen gesetzlichen Zwangs auf eine Minderung steuerlicher Leistungsfähigkeit zu schließen ${ }^{31}$. Sind Zwangsbeiträge als Vermögensbildung zu klassifizieren, so läßt sich lediglich eine Minderung der gegenwärtigen Steuerzahlungsmöglichkeiten konstatieren, was für eine Steuerstundung sprechen könnte; sind Zwangsbeiträge dem Konsumbereich zuzuordnen, so liegt keine Minderung der steuerlichen Leistungsfähigkeit vor. Allerdings treten in beiden Fällen Zusatzlasten (Excess burden) auf, die jedoch im Rahmen der Einkommensermittlung nicht berücksichtigt werden können 32 . Unproblematisch ist die steuerliche Behandlung von Zwangsbeiträgen lediglich dann, wenn diese als Einkunftserzielungskosten eingestuft werden können, so daß ein Abzug von der Steuerbemessungsgrundlage geboten ist.

31 So allerdings im wesentlichen Lamers (1975, 72-75, 80), Söhn (1985, 404), Birk (1986b, 137f.).

$32 \mathrm{Vgl}$. Kapitel II.2.2. 
Haller (1981a, 60) ${ }^{33}$ schlägt vor, den Anteil der Zwangsbeiträge zu bestimmen, der auch freiwillig geleistet worden wäre. Lediglich die Differenz wäre dann als Einschränkung der Dispositionsmöglichkeiten des Steuerpflichtigen anzusehen, so daß sich allenfalls für diesen Teil ein Abzug von der Bemessungsgrundlage vertreten ließe. In der Praxis ist allerdings keine individuelle Regelung möglich, der Gesetzgeber müßte aufgrund von Plausibilitätsüberlegungen pauschale Anteile, evtl. differenziert nach Einkommens- und Familienverhältnissen, vorgeben. $\mathrm{Zu}$ regeln wäre auch, ob die entsprechenden Beiträge definitiv steuerfrei bleiben sollen oder ob deren Steuerpflicht nur aufgeschoben wird. Ein unvermeidbarer Widerspruch zur sonstigen Vorgehensweise, wo allein auf die Frage des objektiv feststellbaren Vermögenszuwachses abgestellt wird, bleibt jedoch bei dieser die Präferenzen der Steuerpflichtigen einbeziehenden Differenzierung. Daher ist eine solche Vorgehensweise nicht praktikabel.

Eine andere Möglichkeit, dem Zwangscharakter von Beiträgen Rechnung zu tragen, besteht darin, über staatliche Zuschüsse (Prämien) zu Beiträgen oder Leistungen von Zwangsversicherungen zu erreichen, daß deren "Rentabilität" (mindestens) derjenigen von freiwillig wählbaren Alternativen entspricht. Im Gegensatz zur oben dargestellten Argumentation wird hier nicht auf den Zwang zur Wahl einer bestimmten Einkommensverwendung an sich (und damit auf die subjektiven Folgen) abgestellt, sondern auf den relativ gut bestimmbaren unmittelbaren wirtschaftlichen Nachteil (Einbuße an ökonomischer Verfügungsmacht) durch die Ausübung staatlichen Zwangs ${ }^{34}$. Relevant sind diese Überlegungen vor allem für den Sozialversicherungszwang, da hier staatliche und private Versicherungsinstitutionen verglichen werden können. Ein ex ante zu bestimmender Rentabilitätsnachteil von Sozialversicherungen läßt sich auf individuelle Faktoren (Steuercharakter von Beiträgen durch interpersonale Umverteilung innerhalb eines Versichertenjahrgangs), auf demographi-

33 Ähnlich auch die Argumentation bei Andel (1970, 331f.; 1980, 373f.), der für einen partiellen Abzug von der Bemessungsgrundlage oder für eine Reduktion des anzuwendenden Steuersatzes plädiert.

34 Dies entspricht weitgehend der Bestimmung eines "Steueranteils", wie er oben dargestellt wurde. 
sche Faktoren (Steuercharakter von Beiträgen durch intergenerationale Umverteilung) sowie auf alle Mitglieder einer Sozialversicherung treffende institutionelle Faktoren (mögliche organisatorische Nachteile, die sich in den Verwaltungskosten niederschlagen) zurückführen. Die Mindestforderung für die einkommensteuerliche Behandlung besteht darin, den Wert des nicht durch andere Maßnahmen ausgeglichenen "Rentabilitätsnachteils" von Zwangsversicherungen durch Abzug von der Steuerbemessungsgrundlage von der Einkommensteuer zu befreien. Dies kann in dem Maße entfallen, wie Zwangsversicherungen beitragsäquivalent ausgestaltet werden 35 .

Eine Gleichstellung freiwilliger Beiträge mit Zwangsbeiträgen, sofern sie dem gleichen Zweck dienen, ist als allgemeiner Grundsatz nicht gerechtfertigt. Die Vergleichbarkeit der jeweiligen Aufwendungen miteinander und das Vorliegen faktischen Zwangs ist im Einzelfall zu prüfen. Eine Ausnahme bilden lediglich sogenannte Quasizwangsbeiträge (Wissenschaftlicher Beirat beim BMF, 1967, 343), die bereits erbrachte Zwangsbeiträge bis zum Erreichen einer gesicherten Anwartschaft aufstocken, sie damit vor einem Verfall schützen und somit ebenfalls rechtlich-institutionell bedingt sind, auch wenn lediglich ein wirtschaftlicher Zwang vorliegt.

Im deutschen Einkommensteuergesetz findet sich eine Auseinandersetzung mit dem Begriff der Zwangsläufigkeit in § 33(2). Die dort anzutreffende Bestimmung der Zwangsläufigkeit von Aufwendungen ist zunächst wenig konkret und damit unangemessen weit gefaßt, wenn auf rechtliche, tatsächliche oder sittliche Gründe verwiesen wird. Eine angemessene sachliche Eingrenzung erfolgt vor allem durch das Kriterium der "Notwendigkeit" (Lang, 1988, 584-597).

\subsection{Meritorische Aspekte}

Meritorische Aspekte lassen sich immer dann anführen, wenn aus gesamtgesellschaftlicher Sicht die individuellen Präferenzen der Bürger

35 Allerdings bleiben bei einer solchen Betrachtung von Veränderungen der ökonomischen Verfügungsmacht mögliche Excess burden außer Betracht. 
bzw. Steuerzahler als suboptimal bezeichnet werden können 36 . Diese Aspekte rechtfertigen dann interventionistische Eingriffe des Staates, sei es durch Ge- und Verbote oder durch finanzielle Anreize ${ }^{37}$. Im Rahmen dieser Arbeit ist von Interesse, wie aus einkommensteuersystematischer Sicht die (finanzielle) staatliche Förderung bestimmter Verhaltensweisen, insb. auch von bestimmten Transferzahlungen, zu beurteilen und in der Bemessungsgrundlage der Einkommensteuer zu berücksichtigen ist.

Liegen bestimmte Verhaltensweisen bzw. Einkommensverwendungen im besonderen Interesse der Allgemeinheit, dann ist als Förderungsinstrument eine Prämie bzw. ein prozentualer Abzug des Verwendungsbetrags von der Steuerschuld zu wählen, nicht jedoch ein Abzug von der Einkommensteuerbemessungsgrundlage ${ }^{38}$. Da hier eine Objektförderung angestrebt wird (im Gegensatz zur in der Einkommensteuer üblichen subjektbezogenen Betrachtung), ist eine gleichmäßige Förderung nur so zu gewährleisten 39 . Fragen der individuellen Leistungsfähigkeit sind nicht berührt, daher ist ein Abzug von der Bemessungsgrundlage der Einkommensteuer nicht angemessen. Ziel der Objektförderung ist es ja gerade, die unter Leistungsfähigkeitsgesichtspunkten ermittelten Steuerbeträge direkt über steuerliche oder indirekt über außersteuerliche Maßnahmen zu korrigieren.

Solche Prämien, ob in Form offener Auszahlungen oder in Form von Abzügen von der Steuerschuld, erhöhen wiederum die steuerliche

36 Vgl. für diese "klassische" Definition Head (1966), für eine kritische Auseinandersetzung mit dem Konzept der meritorischen Güter Andel (1984).

37 Dies allerdings unter der Voraussetzung, daß ein "Staatsversagen" relativ bedeutungslos bleibt.

38 Vgl. Peffekoven (1971/72, 414-417) und Paqué (1986, 344-349).

39 Die Überlegungen lassen sich ebenfalls auf demeritorische Aspekte anwenden. Damit wird beispielsweise eine - allerdings zunächst nur auf konzeptioneller Ebene - systematische Einbeziehung von Glücksspielgewinnen und -verlusten in die Einkommensteuer ermöglicht (vgl. zum Stand der Diskussion Andel, 1980, 355, sowie Lang, 1988, 271f.), indem für steuerliche Zwecke die Aufwendungen um einen bestimmten Prozentsatz gekürzt werden (Negativprämie) bei gleichzeitiger Normalbesteuerung der Gewinne. 
Leistungsfähigkeit des Empfängers. Dies spricht dafür, auch solche staatliche Transfers in die Bemessungsgrundlage der Einkommensteuer aufzunehmen. Dem steht allerdings das Interesse an einer gleichmäßigen Förderung bestimmter Verhaltensweisen bzw. Einkommensverwendungen gegenüber, für das ebenfalls Gerechtigkeitsargumente und darüber hinaus Allokationsaspekte angeführt werden können. Das Kalkül der Steuerpflichtigen soll im gleichen Ausmaß verändert werden: Ist die Bemessungsgrundlage der Prämie eine bestimmte Art der Einkommensverwendung (z.B. ein Spendenbetrag), also Einkommen nach Steuern, dann muß eine je gleiche prozentuale Begünstigung als solche steuerfrei bleiben, weil sonst die Nettoprämien zwischen einzelnen Steuerpflichtigen differieren würden 40 . Wird berücksichtigt, daß es gerade das Ziel solcher Prämien ist, die Steuerbemessung nach der Leistungsfähigkeit zu verändern, daß ihre Höhe zudem unter der Kontrolle des Gesetzgebers ist und daß darüber hinaus keine zusätzlichen individuellen Steuervermeidungsspielräume geschaffen werden, so scheint es vertretbar, solche Prämien von der Steuerpflicht auszunehmen 41 .

$\mathrm{Zu}$ beachten ist dann allerdings, daß das steuerliche Einkommen nicht den tatsächlichen Zuwachs an ökonomischen Verfügungsmöglichkeiten widerspiegelt. Für Zwecke einer Bedarfsüberprüfung, wie sie vor der Gewährung verschiedener staatlicher Transfers üblich ist, ist daher neben

40 Anders wäre es, wenn dem Ziel eine mit steigendem Einkommen fallende Prämie entsprechen würde, wie das beispielsweise bei der Sparförderung vorstellbar ist, bei der momentan mit Einkommensgrenzen gearbeitet wird. Bei einer Steuerpflicht hängt die Höhe der Nettoprämie allerdings direkt vom Tarifverlauf ab, der die (Netto-)Relationen zwischen verschiedenen Steuerpflichtigen festlegt. Auch Tarifänderungen im Zeitverlauf wirken sich dann automatisch auf die Höhe der Förderung aus.

$41 \mathrm{Vgl}$. aus juristischer Sicht Lang $(1987,26)$. Auch Überlegungen auf der Nutzenebene sprechen für eine solche Lösung: Durch die Prämiengewährung soll eine Änderung von Verhaltensweisen erreicht werden, sollen Substitutionseffekte erzielt werden, so daß im Extremfall nur ein marginaler Vorteil aus der Prämiengewährung verbleibt. 
dem zu versteuernden Einkommen der nachträgliche Ausweis eines "verfügbaren Einkommens" zu erwägen ${ }^{42}$.

Die Höhe von Förderprämien läßt sich unter Leistungsfähigkeitsgesichtspunkten nicht bestimmen. Anhaltspunkte kann die Stärke des Gemeininteresses in Relation zum Privatinteresse liefern, womit sich rein theoretisch "Förderprämien" zwischen 0\% (kein öffentliches Interesse) und $100 \%$ (volle staatliche Kostenübernahme) begründen lassen. $\mathrm{Zu}$ beachten ist auch, ob der Umfang einer Förderung bestimmter freiwilliger Einkommensverwendungen im Vergleich zu den Nettoeffekten steuerlich anerkannter Leistungsfähigkeitsminderungen, die allerdings Ergebnis einer völlig anderen Konzeption sind, vertretbar ist ${ }^{43}$.

Während eine Leistungsfähigkeitsminderung selbst dann, wenn sie sich durch verschiedene Argumente gleichzeitig begründen läßt, nur einmal zu einer Reduktion der Steuerbemessungsgrundlage führen kann, ist bei staatlichen Förderungen auch eine Kumulation von Förderprämien möglich, sofern die Kriterien mehrerer Fördermaßnahmen erfüllt werden (Krupp, 1978, 65f.). Auch eine Prämierung von Aufwendungen, die bereits die steuerliche Bemessungsgrundlage mindern, ist denkbar, wodurch sich Leistungsfähigkeitsüberlegungen mit allgemeinen Förderaspekten verknüpfen lassen.

\section{Methoden der steuerlichen Erfassung von Transferzahlungen}

Dieser Abschnitt beschränkt sich auf die Frage der Erfassung beim Empfänger. Beim Transfergeber kommt unter Leistungsfähigkeitsgesichtspunkten lediglich ein Abzug von der Steuerbemessungsgrundlage

42 Für diese Zwecke sind Korrekturen des zu versteuernden Einkommens auch heute schon üblich und berechtigt (vgl. Giloy, 1978, 69-92; Institut Finanzen und Steuern, 1985; Bundesverfassungsgericht, 1991a, 98-104). Allerdings ist zunächst auch hier eine Entscheidung erforderlich, ob einer gleichmäßigen Förderung im Sinne einer konstanten Nettoprämie oder einer realitätsgerechten Überprüfung von Bedarfsdeckungsmöglichkeiten höhere Priorität eingeräumt werden soll.

43 Vgl. für eine solche Gegenüberstellung Lamers $(1975,62 \mathrm{f}$.). 
in Frage. Ein Abzug von der Steuerschuld würde voraussetzen, daß der Transfergeber anstelle staatlicher Institutionen tätig wird.

\section{(1) Steuerpflicht}

Die Steuerpflicht von Transferzahlungen ist die übliche Form der Berücksichtigung der Erhöhung steuerlicher Leistungsfähigkeit (Lang, 1983, 109; Kausemann, 1983, 162-168; Gaddum, 1986, 33). Formal setzt eine Steuerpflicht die Erfassung innerhalb einer der Einkunftsarten des Einkommensteuergesetzes voraus ${ }^{44}$, ggf. durch die Schaffung einer weiteren Einkunftsart "Persönliche Einnahmen" bzw. "Privatbezüge" (Lang, 1985, 98f.; 1988, 537-542).

\section{(2) Anrechnungsverfahren}

Beim Anrechnungsverfahren werden Transferzahlungen mit dem gleichen Zweck dienenden einkommensteuerlichen Abzugsbeträgen verrechnet. Es ist insofern mit einer Steuerpflicht identisch, als die leistungsfähigkeitserhöhende Wirkung von Transferzahlungen auch hier berücksichtigt wird - allerdings nur bis zu einer Obergrenze, die durch einkommensteuerliche Bestimmungen zur Steuerfreiheit bestimmter Aufwendungen, z.B. für den besonderen Bedarf von Behinderten oder auch für den Grundbedarf in Form des Existenzminimum-Grundfreibetrags, festgelegt wird. Das Anrechnungsverfahren läßt sich daher nur anwenden, wenn eine besondere Zweckbestimmung von Transfers vorliegt ${ }^{45}$ und Aufwendungen für diesen Zweck innerhalb der Einkommensteuer als Reduktion der Steuerbemessungsgrundlage zu berücksichtigen sind 46 .

$44 \mathrm{Vgl}$. hierzu auch die doppeldeutige Beschlußfassung des Deutschen Juristentags (1988, N213), der sich für eine Steuerpflicht "im tatbestandlichen Rahmen der Einkunftsarten" ausspricht.

45 Vgl. dazu auch Lang $(1988,542-545)$.

46 Dies gilt auch für den Grundfreibetrag, der lediglich als Tarifbestimmung falsch plaziert ist. Vgl. dazu Haller (1981b, 12-14), Dziadkowski (1986, 508f.) und Karl-Bräuer-Institut des Bundes der Steuerzahler (1991, 81f.). Bei einer formalen Anordnung im Rahmen der Ermittlung der Steuerbemessungsgrundlage wären dann auch die hier angesprochenen Korrekturen möglich. 
Die Anrechnung bewirkt dann, daß einkommensteuerlich als Reduktion der Steuerbemessungsgrundlage berücksichtigungsfähige Pauschalen oder tatsächliche Aufwendungen um den jeweiligen dem gleichen Zweck dienenden Transferbetrag gekürzt werden, so daß sich im Extremfall - der Transferbetrag entspricht den einkommensteuerlich anzusetzenden Beträgen oder übersteigt diese - einkommensteuerliche Berücksichtigung und Anrechnung von Transfers gerade kompensieren.

Von der unter (1) beschriebenen expliziten Steuerpflicht von Transferzahlungen unterscheidet sich dieses Verfahren auch dadurch, daß ausdrücklich ausgeschlossen wird, daß die Transferzahlungen für sich betrachtet eine Steuerpflicht auslösen. Sachlich gerechtfertigt kann die Anwendung dieses Verfahrens dann sein, wenn einkommensteuerlich vorgesehene Bedarfspauschalen sich in Einzelfällen als unzureichend erweisen 47 .

Die unter Steuerrechtlern geführte Diskussion um die Frage der Steuerpflicht staatlicher Transferzahlungen läßt sich weitgehend auf eine Kontroverse um die Modalitäten der Anwendung des hier beschriebenen Anrechnungsverfahrens reduzieren: Soll für jeden leistungsfähigkeitsmindernden Tatbestand separat mit öffentlichen Leistungen saldiert werden (Kirchhof, 1988, F66), was insbesondere im Grundbedarfsbereich Probleme der Zuordnung aufwirft, oder die Summe privater Bezüge auf die Summe privater Abzüge angerechnet werden (Lang, 1985, 71, 98f.; 1987, 25f.).

\section{(3) Progressionsvorbehalt}

Beim Verfahren des Progressionsvorbehalts (vgl. § 32b EStG) bleiben die einbezogenen Transferzahlungen steuerfrei, werden allerdings zur Bestimmung des auf weitere Einkünfte anzuwendenden Steuersatzes in die Steuerbemessungsgrundlage einbezogen 48 . Bei einer Anwendung des

47 Für Steuerpflichtige, deren Bedarf nicht durch Transferzahlungen gedeckt wird, kann dies allerdings eine einkommensteuerliche Benachteiligung zur Folge haben. $\mathrm{Zu}$ systembedingten Unterschieden der Bedarfsermittlung vgl. allerdings Kapitel IV.1.2.

48 Rechtssystematische Probleme sieht Giloy (1982, 130), weil damit dann doch eine steuererhöhende Wirkung erzielt wird. 
Progressionsvorbehalts erfolgt eine einkommensteuerliche Entlastung nach Maßgabe des individuellen Durchschnittssteuersatzes, während bei einer Steuerbefreiung ohne Anwendung dieser Regelung nach Maßgabe des individuellen Grenzsteuersatzes auf Einkommensteuereinnahmen verzichtet wird. Im Gegensatz zum Anrechnungsverfahren (2) kann der Progressionsvorbehalt auch auf Transfers angewandt werden, die - wie z.B. Lohnersatzleistungen - eine allgemeine Erhöhung der Leistungsfähigkeit des Empfängers zum Ziel haben. Implizit wird mit der Anwendung des Progressionsvorbehalts die Steuerautonomie über die betreffenden Einkommensteile an eine andere Körperschaft abgetreten. So wird bei Sozialversicherungstransfers i.d.R. ein pauschalierter fiktiver Steuerabzug im Rahmen der Transferfestsetzung vorgenommen.

Sowohl die pauschale Kürzung von Transfers um einen fiktiven oder tatsächlichen Steuerabzug als auch die Heranziehung der Transfers zur Bestimmung eines auf weitere Einkünfte anzuwendenden Durchschnittssteuersatzes verhindern allerdings eine konsistente Leistungsfähigkeitsbesteuerung. 
Harald Schlee - 978-3-631-75216-6

Downloaded from PubFactory at 01/11/2019 06:59:36AM

via free access 


\section{DIREKTE STAATLICHE TRANSFERS}

\section{Sozialhilfe}

\subsection{Charakterisierung}

Den Empfängern von Sozialhilfe soll die Führung eines menschenwürdigen Lebens ermöglicht werden (§1 BSHG). Vor der Gewährung von Sozialhilfe ist allerdings eine Bedürftigkeitsprüfung vorzunehmen, in der festgestellt wird, ob ein Antragsteller zur Selbsthilfe in der Lage ist oder Ansprüche auf Hilfe Dritter bestehen ("Nachrang der Sozialhilfe"; § 2 BSHG). Die Verpflichtung zur Selbsthilfe schließt den Einsatz der eigenen Arbeitskraft mit ein 1 . Die Sozialhilfe wird als Hilfe zum Lebensunterhalt, die der Deckung eines allgemeinen Grundbedarfs dienen soll, oder als Hilfe in besonderen Lebenslagen gewährt.

Die Hilfe zum Lebensunterhalt umfaßt Leistungen, die dazu bestimmt sind, dem Empfänger den notwendigen Lebensunterhalt - vor allem Ernährung, Unterkunft, Kleidung - zu ermöglichen. Ein Teil des Regelbedarfs wird dabei in Form von Regelsätzen pauschaliert (§ 22 BSHG, $\S 1$ Regelsatzverordnung), als Aufwendungen für Unterkunft werden i.d.R. die tatsächlichen Aufwendungen anerkannt (§3 Regelsatzverordnung); hinzu kommen insbesondere Leistungen für unregelmäßig auftretenden Bedarf. Für besondere Personengruppen (Alte, Behinderte,

1 Vgl. zur Umsetzung dieser Bestimmung Schulte/Trenk-Hinterberger (1986, 196-218). Wird die Übernahme einer zumutbaren Arbeit abgelehnt, kann die Sozialhilfe auf den "unerläßlichen Lebensunterhalt", der etwa in Höhe von $80 \%$ der Regelsätze angegeben wird, eingeschränkt und in der Form von Geld- auf Sachleistungen umgestellt werden. Der Reformvorschlag von Leibfritz/Parsche (1988, VI'), der eine Kürzung des Anspruchs auf 50\% für erwerbsfähige, jedoch nicht erwerbstätige Personen vorsieht, ist daher aus verfassungsrechtlichen Gründen nicht umsetzbar. Die bei einer Realisierung des Vorschlags zunehmende Attestierung von Erwerbsunfähigkeit würde zudem den Zustand der Erwerbslosigkeit der betroffenen Personen eher festschreiben und damit der Zielsetzung einer Erhöhung der Arbeitsanreize zuwiderlaufen; schließlich würden nicht erwerbsfähige Familienangehörige von einer solchen Maßnahme unweigerlich mitgetroffen. 
Erwerbsunfähige, Erwerbstätige, Alleinerziehende, werdende Mütter; $\S 23$ und § 24 BSHG) ist die Anerkennung eines regelmäßig auftretenden zusätzlichen Bedarfs ("Mehrbedarf") vorgesehen.

Die Hilfe in besonderen Lebenslagen soll besonderen Belastungssituationen Rechnung tragen, z.B. in Form der Pflegehilfe, Altenhilfe oder Krankenhilfe. Während im Rahmen der Hilfe zum Lebensunterhalt grundsätzlich von einem vollen Einsatz eigenen Einkommens und Vermögens sowie der eigenen Arbeitskraft ausgegangen wird und auch Dritte soweit möglich - in starkem Maße herangezogen werden, wird für die Hilfe in besonderen Lebenslagen eine Selbsthilfe nur verlangt, sofern sie als "zumutbar" angesehen wird ( $\S 28,79-87$ BSHG). Damit wird die Gewährung von Hilfe in besonderen Lebenslagen auf einen Personenkreis ausgeweitet, der in der Lage wäre neben seinem Grundbedarf (entsprechend der Abgrenzung der Hilfe zum Lebensunterhalt) auch den durch die besondere Lebenssituation bedingten Bedarf zum Teil oder sogar vollständig selbst zu tragen.

\subsection{Einkommensteuersystematische Aspekte}

Da sowohl die Sozialhilfe als auch die Einkommensteuer auf das Existenzminimum Bezug nehmen, ist sicherzustellen, daß alle Leistungen der Sozialhilfe auch nach Maßgabe des Einkommensteuergesetzes steuerfrei bleiben. Damit wird erreicht, daß zum einen Sozialhilfeempfänger auf die ihnen als Existenzminimum gewährten Leistungen auch bei einer umfassenden Steuerpflicht von Transfereinkommen keine Einkommensteuer zu zahlen haben (die vom Sozialhilfeträger übernommen werden müßte) ${ }^{2}$, zum anderen auch alle Steuerpflichtigen, deren Einkommen oberhalb des Sozialhilfeniveaus (Existenzminimums) liegt - und die daher ihre existentiellen Bedürfnisse aus eigener Kraft zu finanzieren in der

2 Vgl. zu diesem Problem bei Bezug von Arbeitslosengeld bzw. -hilfe A.Prinz (1986). 
Lage sind - bezüglich des Umfangs des ihnen zu gewährenden Existenzminimums mit Sozialhilfeempfängern mindestens ${ }^{3}$ gleichgestellt werden.

Sofern diesem Gedanken im Grundsatz zugestimmt wird, sind allerdings noch einige Besonderheiten zu beachten:

Unregelmäßig auftretender Bedarf, z.B. in Form von größeren Anschaffungen, führt in der Sozialhilfe zu einmaligen Leistungen, da Rücklagen für diese Zwecke nicht vorhanden sind. In der Einkommensteuer ist es dagegen zweckmäßig, solche Aufwendungen im Rahmen des Existenzminimum-Grundfreibetrags pauschalierend in ihrer jahresdurchschnittlichen Höhe zu berücksichtigen, weil damit eine Einzelfallprüfung vermieden werden kann. Insofern können sich bei kurzfristiger Betrachtung die Existenzminima in der Sozialhilfe einerseits und der Einkommensteuer andererseits - selbst bei prinzipiell gleichen Vorstellungen über einen zugrunde zu legenden Warenkorb - unterscheiden. Diese Abweichungen gleichen sich im Normalfall längerfristig wieder aus. Lediglich bei einzelnen Steuerpflichtigen kann durch temporären Bezug von Sozialhilfeleistungen und anschließende pauschale einkommensteuerliche Berücksichtigung bereits durch die Sozialhilfe gedeckten Bedarfs eine zweifache Begünstigung eintreten; allerdings ist auch der umgekehrte Fall einer zu geringen Bedarfsberücksichtigung denkbar, wenn ein Anschaffungszeitpunkt nicht in den Zeitraum des Sozialhilfebezugs fällt ${ }^{4}$.

Weitere Differenzen zwischen Sozialhilfe und einkommensteuerlichem Existenzminimum ergeben sich dann, wenn im Rahmen der Sozialhilfe tatsächliche Kosten erstattet werden, im Rahmen der Einkom-

3 Es ist vertretbar, wenn innerhalb der Einkommensteuer ein etwas höherer Lebensstandard für das Existenzminimum zugrundegelegt wird als bei den voll staatlich finanzierten Leistungen der Sozialhilfe, nicht jedoch umgekehrt. Vgl. Kirchhof (1988, F60) sowie Deutscher Juristentag (1988, N215).

$4 \mathrm{Da}$ in der Sozialhilfe vor allem auf Zahlungsvorgänge abgestellt wird, ergeben sich solche konzeptionellen Abweichungen auch auf der Einnahmenseite. Diese sind jedoch unproblematisch, da Zurechnungen zum steuerlichen Einkommen lediglich eine ungerechtfertigte Inanspruchnahme der Sozialhilfe verhindern. 
mensteuer aber Pauschalierungen herangezogen werden 5 . Ein erster Schritt zur Reduzierung solcher Unterschiede liegt in der möglichst realitätsgerechten Festsetzung von Pauschalen, ggf. auch unter Berücksichtigung regionaler Unterschiede6. Darüber hinaus liegt der Gedanke nahe, dann noch verbleibenden Abweichungen durch eine großzügige Bemessung des Einkommensteuer-Grundfreibetrags Rechnung zu tragen, um damit Benachteiligungen von Steuerpflichtigen gegenüber Sozialhilfeempfängern sowie eine mögliche Steuerpflicht von Teilen der Sozialhilfe auszuschließen.

In diesen Fällen einer grundsätzlichen konzeptionellen Übereinstimmung zwischen Sozialhilfe und Einkommensteuer wäre eine Steuerpflicht für die ausgezahlte Sozialhilfe offensichtlich zielinadäquat: Steuerzahlungsverpflichtungen würden sich allenfalls aufgrund von im Einzelfall unzutreffenden Pauschalierungen oder zeitlichen Zurechnungen ergeben. Das Verfahren der Anrechnung gezahlter Sozialhilfe auf den Einkommensteuer-Grundfreibetrag und ggf. auf besondere Freibeträge bei einkommensteuerlich anerkanntem Mehrbedarf (Lang, 1987, 26) schließt solche unbeabsichtigte Wirkungen bei Wahrung größtmöglicher steuersystematischer Einbindung aus; in den genannten Fällen führt allerdings das Anrechnungsverfahren nicht $\mathrm{zu}$ anderen Ergebnissen wie eine explizite Steuerfreiheit von Sozialhilfeleistungen.

Anders verhält es sich in den Sonderfällen der Sozialhilfe, in denen von der sonst vorrangigen Verpflichtung des Einsatzes eigenen Einkommens abgegangen wird und stattdessen besondere Zumutbarkeitsregelungen etabliert werden:

Dies ist zum einen beim Mehrbedarf von Erwerbstätigen der Fall, der für die Personengruppe des § 23(4) BSHG bis zu 50\% bzw. 66,66\% des Regelsatzes eines Haushaltsvorstandes betragen, für die Personengruppe des § 24 BSHG noch darüber hinausgehen kann (Brühl, 1989, 28-31) und

5 Dies ist letztlich darauf zurückzuführen, daß bei der Sozialhilfegewährung aufgrund der Einzelfallbetrachtung eine bessere Datenlage vorhanden ist als in der Einkommensteuer, die aus verfahrenstechnischen Gründen auf Pauschalierungen bei beschränkter Information zurückgreifen muß.

6 Vgl. Kapitel II.2.8. 
eine Erhöhung des Arbeitsanreizes bewirken soll. Sowohl bei einer Steuerpflicht von Sozialhilfeleistungen als auch beim Anrechnungsverfahren kann sich hier eine positive Steuerschuld ergeben, und zwar dann, wenn die Sozialhilfeleistungen zuzüglich der Erwerbseinkünfte (einschl. Werbungskosten) höher als Grundfreibetrag der Einkommensteuer und einkommensteuerliche Werbungskosten bzw. Werbungskostenpauschale sind. Hier ist es nicht einleuchtend, daß die entsprechenden Sozialhilfebeträge steuerfrei bleiben sollten: Auch in der Einkommensteuer sind Werbungskosten, ggf. ergänzt durch eine pauschale Berücksichtigung lediglich indirekt einer Erwerbstätigkeit zuzurechnender Kosten, zu berücksichtigen.

Ein zweiter Bereich, in dem vom vollen Einsatz des eigenen Einkommens Abstand genommen wird, sind spezielle Einkommensgrenzen für die Gewährung von Hilfe in besonderen Lebenslagen. Damit wird in bestimmten Bedarfssituationen Sozialhilfe auch dann geleistet, wenn nach dem sonst im Rahmen der Sozialhilfe verwandten Leistungsfähigkeitsmaßstab, der grundsätzlich dem der Einkommensteuer entspricht und sowohl einen Grundbedarf als auch besondere Bedarfssituationen berücksichtigt, ein Bedarf nicht besteht. Für die Gewährung von Sozialhilfe in besonderen Lebenslagen wird somit ein zweiter Leistungsfähigkeitsmaßstab eingeführt. Nach dem Leistungsfähigkeitsprinzip ist die unter Anwendung besonderer Einkommensgrenzen gewährte Hilfe in besonderen Lebenslagen zum steuerpflichtigen Einkommen zu rechnen, da die besondere Bedarfssituation einkommensteuerlich bereits berücksichtigt ist, die Sozialhilfezahlung somit die steuerliche Leistungsfähigkeit erhöht (Steuerpflicht der Sozialhilfe), oder, anders ausgedrückt, die für die besondere Lebenslage einkommensteuerlich geltend gemachten Aufwendungen um die Kostenübernahme durch die Sozialhilfe zu kürzen sind, da lediglich die verbleibende Belastung effektiv wird (Anrechnungsverfahren). Auch wenn von einem Willen des Gesetzgebers zur (Netto-) Förderung der betroffenen Personengruppen - die sich durch die gegebene Bedarfssituation und "niedrige", wenn auch ausreichende Einkommen auszeichnen - ausgegangen wird, ändert sich wegen der Zweckgleichheit der Sozialhilfe und besonderer Freibeträge der Einkommensteuer an 
diesen Schlußfolgerungen nichts. Jedenfalls beim Anrechnungsverfahren7 wird lediglich eine zweifache Berücksichtigung der Bedarfssituation aufgehoben. Sonst würden steuerpflichtige weitere Einkünfte in Höhe der steuerlichen Abzugsbeträge unberechtigterweise steuerfrei bleiben, obwohl sie zur freien Verwendung für Ausgaben oberhalb eines Grundbedarfs zur Verfügung stehen. Denn eine tatsächliche Belastung tritt nach einer Kostenübernahme durch die Sozialhilfe nicht ein.

Es drängt sich die Frage auf, ob wegen der vielfältigen Notwendigkeiten einer Abstimmung zwischen Sozialhilfe und Einkommensteuer sowohl im Rahmen der Gesetzgebung (Bestimmung eines sozio-kulturellen Existenzminimums) als auch in der Praxis der Sozial- und Finanzämter - eine einzige Institution sowohl für Sozialhilfeleistungen als auch für die Erhebung der Einkommensteuer zuständig sein sollte (vgl. Kausemann, 1983, 360-372). Wegen der notwendigen Abstimmung verschiedener Formen der Hilfeleistung auf die Situation des einzelnen Hilfesuchenden (Geldleistungen, Sachleistungen, persönliche Hilfe; vgl. Schulte/Trenk-Hinterberger, 1986, 141-143) und der Möglichkeit hierfür beim Sozialhilfeträger einen Ansprechpartner zur Verfügung zu stellen und damit eine laufende intensive Betreuung anzubieten, sollte meiner Ansicht nach die vorhandene Institutionentrennung aufrechterhalten werden. Die oben dargestellte allein auf die Geldleistungen beschränkte Sichtweise wird den umfassenderen Aufgabenstellungen nicht gerecht. Allerdings könnte das Finanzamt als zentrale Erfassungsstelle von Transfers, soweit unbare Zahlungen geleistet werden auch als Zahlstelle, dienen.

Auch wenn rein steuersystematische Aspekte eine Steuerpflicht für Sozialhilfeleistungen nahelegen, sprechen doch gewichtige verfahrenstechnische Argumente lediglich für die Einführung eines Anrechnungs-

7 Bei expliziter Steuerpflicht könnte es wiederum zu unbeabsichtigten Benachteiligungen einzelner Steuerpflichtiger kommen, wenn im konkreten Einzelfall aufgrund der angesprochenen verfahrenstechnischen Unterschiede Differenzen zwischen sozialhilferechtlich und einkommensteuerlich anzuerkennenden Beträgen auftreten. 
verfahrens von Sozialhilfeleistungen auf jeweils dem gleichen Zweck dienende einkommensteuerliche Freibeträge. Dies gilt ebenfalls, sofern tatsächliche Aufwendungen einkommensteuerlich relevant sind. Die Ursache hierfür liegt in den teils behebbaren, teils systemgegebenen Abstimmungsproblemen zwischen Steuer- und Sozialhilferecht bezüglich Höhe und Zeitpunkt der Berücksichtigung von Aufwendungen. Denn nur bei einer völligen Identität der jeweiligen Bestimmungen ist eine explizite Steuerpflicht von Sozialhilfeleistungen inhaltlich gerechtfertigt und den betroffenen Steuerpflichtigen gegenüber vertretbar. Das Anrechnungsverfahren vermeidet dagegen eine Steuerpflicht aus Sozialhilfeleistungen in jedem Einzelfall, kann allerdings eine Steuerpflicht für weitere Einkünfte begründen. Dabei wird eine zweifache Berücksichtigung von Bedarfslagen weitgehend - abgesehen von in Einzelfällen effektiv werdenden Unterschieden in der zeitraumbezogenen Zurechnung im Steuer- und Sozialhilferecht - vermieden. Die Höhe des effektiv einkommensteuerlich berücksichtigten Bedarfs wird dabei durch den jeweils höheren Betrag aus Einkommensteuerrecht einerseits, Sozialhilferecht andererseits determiniert.

Für die Anpassung der Rechtslage in den beiden Bereichen sollten die im Rahmen der Sozialhilfe und der Einkommensteuer als Grundbedarf anerkannten Beträge laufend verglichen werden. Eine Indikatorfunktion für eine ausreichende Berücksichtigung des Existenzminimums innerhalb der Einkommensteuer kommt dabei einer fiktiven Steuerveranlagung von ausgewählten Modellfällen mit steuerpflichtigen Einkünften in Höhe des jeweiligen Sozialhilfeanspruchs zu.

\section{Wohngeld und weitere wohnungsbezogene Transfers}

\subsection{Charakterisierung}

Wohngeld wird als Zuschuß zur "wirtschaftlichen Sicherung angemessenen und familiengerechten Wohnens" ( $§ 1$ WoGG) gezahlt. Ziel ist es, tragbare Wohnkostenbelastungen zu erreichen ${ }^{8}$. Dabei werden sowohl die

8 Vgl. Bundesminister für Arbeit und Sozialordnung $(1990,83)$. 
Belastungen durch Mietzahlungen (Mietzuschuß) als auch diejenigen von Eigentümern selbstgenutzten Wohnraums durch Zinsen und Tilgung (Lastenzuschuß) berücksichtigt. Miet- bzw. Lastenzuschuß sind in Abhängigkeit von der Familiengröße, dem Familieneinkommen sowie der Höhe der Miete bzw. Belastung festgelegt. Für die berücksichtigungsfähige Miete bzw. Belastung gelten Höchstbeträge, die nach regionalen Gesichtspunkten sowie nach Ausstattung und Fertigstellungszeitpunkt der Wohnung differenziert sind. Sofern ein zum Haushalt gehörendes Familienmitglied im Antragsjahr vermögensteuerpflichtig ist, wird die Gewährung von Wohngeld grundsätzlich versagt. Laut $\S 3 \mathrm{Nr} .58 \mathrm{EStG}$ ist das Wohngeld explizit von der Einkommensteuer befreit.

Neben dem Wohngeld als direkt subjektbezogener Fördermaßnahme bestehen noch eine Reihe weiterer stärker objektbezogene staatliche Transfers mit wohnungspolitischer Zielsetzung. So werden auf der Grundlage des Zweiten Wohnungsbaugesetzes der Mietwohnungsbau sowie Wohneigentumsmaßnahmen für bestimmte Personengruppen durch Zuschüsse und zinsgünstige Kredite gefördert ${ }^{9}$. Hinzu kommen Förderungen in Form von Steuervergünstigungen, insbesondere durch Sonderabschreibungen nach $\S \S 10 \mathrm{e}, 10 \mathrm{f}, 10 \mathrm{~h}$ EStG und günstige Abschreibungsmodalitäten nach $\S \S 7-7 \mathrm{c}, 7 \mathrm{k}$ EStG.

\subsection{Einkommensteuersystematische Aspekte}

Während das von der Sozialhilfe gewährte Existenzminimum konzeptionell im Regelfall mit dem Existenzminimum-Grundfreibetrag der Einkommensteuer, evtl. aufgestockt durch zusätzliche (Sonder-)Freibeträge entsprechend der individuellen Situation der Steuerpflichtigen, übereinstimmt, ist beim Wohngeld eine solche systematische Verknüpfung mit der Einkommensteuer nicht möglich. Der Ausgabenbedarf für Wohnen ist - jedenfalls von der Konzeption her - bereits im Existenzminimum-

9 In der Wohnungsbauförderung werden auf Länderebene je nach sozialer Lage des Adressatenkreises bis zu vier sog. Förderwege unterschieden. Diese sind in den einzelnen Bundesländern z.T. unterschiedlich ausgestaltet. 
Grundfreibetrag enthalten ${ }^{10}$. Ein Sonderfreibetrag in Höhe der Wohngeldleistungen ist insoweit nicht angebracht, das Wohngeld damit zu den steuerpflichtigen Einkünften zu rechnen. Eine Belastung der Steuerpflichtigen mit Ausgaben für "Wohnen" liegt im Ausmaß der Wohngeldzahlungen nicht mehr vor, womit Einkommensteile - auch für eine Steuerzahlung - frei verfügbar werden, die vorher als Grundbedarf gebunden waren 11 .

Eine Steuerpflicht des Wohngeldes könnte mit dem Argument abgelehnt werden, das Einkommen gehe bereits in die Berechnung des Wohngeldes ein, prinzipiell sei daher eine Vergabepraxis möglich, die als Ergebnis Nettobeträge auszahle. Eine Vorwegnahme der Einkommensteuerbelastung, und damit eine Orientierung des Wohngelds am zu versteuernden Einkommen, ist jedoch wegen des Zeitbedarfs für Einkommensteuerveranlagung bzw. Lohnsteuerjahresausgleich verfahrenstechnisch nicht möglich. Auch fließen im Rahmen der Wohngeldberechnung Kriterien ein, die sich von denjenigen der Einkommensteuer unterscheiden. Schließlich wird der Leistungsfähigkeitsmaßstab "zu versteuerndes Einkommen" ohne Einbezug des Wohngelds verzerrt wiedergegeben, was Auswirkungen für die Vergabe anderer staatlicher Transfers haben kann.

Allerdings trägt der Einkommensteuer-Grundfreibetrag den in ganz erheblichem Ausmaß nicht individuell zu verantwortenden Differenzen in der Ausgabenbelastung der privaten Haushalte durch Wohnungsausgaben nicht genügend Rechnung. Unter systematischen Aspekten sollte zunächst für alle Steuerpflichtigen eine Differenzierung des einkommensteuerlichen Grundfreibetrags erfolgen. Das Wohngeld kann kein befriedigender Ersatz für ausreichende Freibeträge der Einkommensteuer sein, zumal

10 Entsprechend sieht der Reformvorschlag von Leibfritz/Parsche (1988, 42*) auch eine Abschaffung des Wohngelds vor, womit dessen eigenständige Zielsetzung jedoch nicht mehr berücksichtigt werden kann.

11 Dem entspricht die volle Anrechnung des Wohngeldes auf die Sozialhilfe (vgl. Kausemann, 1983, 382f.). 
diese auch Nicht-Wohngeldberechtigten zu gewähren sind. Im Rahmen einer aus Praktikabilitätsgründen notwendigen Pauschalierung bestehen gute Chancen für die Berücksichtigung allgemeiner regionaler Unterschiede, etwa in Anlehnung an die regionalen Mietenstufen des $\S 8$ WoGG12. Eine Berücksichtigung tatsächlicher individueller Aufwendungen für den Grundbedarf im Bereich "Wohnen" ist jedoch in der Einkommensteuer kaum möglich. Dagegen kann für den Personenkreis der Wohngeldbezieher die tatsächliche Höhe der Aufwendungen berücksichtigt werden. Insofern läßt sich eine einkommensteuerliche Sonderstellung der Wohngeldzahlungen rechtfertigen.

Zur Besteuerung von Wohngeld läßt sich daher abschließend sagen, daß aus einkommensteuersystematischer Sicht eine explizite Steuerpflicht der Wohngeldleistungen bei gleichzeitiger Gewährung eines wohnaufwandsbezogenen Grundfreibetrags naheliegt. Umkippeffekte der Begünstigung durch Wohngeld werden auch durch eine Steuerpflicht nicht verursacht. Aufgrund der stark differierenden Belastungen mit Ausgaben für Wohnzwecke, die zwar im Wohngeld, nicht aber in gleicher Weise in der Einkommensteuer berücksichtigt werden können, läßt sich auch eine Steuerfreiheit des Wohngelds vertreten. Dann ist allerdings die reduzierte Belastung mit Ausgaben für Wohnzwecke ebenfalls im einkommensteuerlichen Grundbedarf, also bei der Bemessung des ExistenzminimumGrundfreibetrags, zu berücksichtigen, was auf die Anwendung des Anrechnungsverfahrens für gezahltes Wohngeld auf den für Wohnzwecke bestimmten Teil des Existenzminimum-Grundfreibetrages hinausläuft.

Die Abwicklung der Wohngeldvergabe könnte im Rahmen der Einkommensteuererklärung vom zuständigen Finanzamt mit betreut werden, da diesem ohnehin die meisten notwendigen Informationen, wie z.B. Angaben zum Einkommen, zum Vermögen und zur Haushaltsgröße vorliegen bzw. (aus Sicht der Steuerpflichtigen) übermittelt werden müssen. Auf freiwilliger Basis könnte ein zusätzlicher Bogen zur Wohnsituation (u.a. Miet- bzw. Belastungshöhe, Ausstattung der Wohnung) durch die Steuerpflichtigen ausgefüllt werden. Damit wäre auch eine

12 Vgl. Kapitel II.2.8. 
deutlich bessere Ausschöpfung des Kreises der Wohngeldberechtigten (Reduzierung der in der Sozialhilfe so genannten verschämten Armut) und somit eine Erhöhung der Transfergerechtigkeit möglich.

Der Mietenvorteil von Sozialwohnungen ist in seinen Auswirkungen auf die ökonomische Dispositionskraft weitgehend mit der Begünstigung durch Wohngeld vergleichbar. Die Höhe eines solchen Vorteils läßt sich bei weitgehend sachgerechter Ausgestaltung einer Fehlbelegungsabgabe durch einen Rückgriff auf deren Regelungen feststellen, da es das Ziel der Fehlbelegungsabgabe ist, Differenzen zwischen "Sozialmiete" und "ortsüblicher Vergleichsmiete" abzuschöpfen. Ein tatsächlich vorhandener Mietenvorteil von Sozialwohnungen läßt sich demnach als Differenz aus maximal möglicher Fehlbelegungsabgabe (z.B. Höchstbetrag nach $\S 8$ und $\S 9$ Hessisches Gesetz zum Abbau der Fehlsubventionierung im Wohnungswesen) und entrichteter Fehlbelegungsabgabe ermitteln; er ist ebenfalls zu den steuerpflichtigen Einkünften zu rechnen. Im Idealfall wird dann als Teil des Existenzminimum-Grundfreibetrages statt der "ortsüblichen Vergleichsmiete" lediglich die tatsächlich gezahlte "Sozialmiete" berücksichtigt.

Die Berücksichtigung von Kreditsubventionen im Rahmen von Kapital- und Aufwendungsdarlehen sowie der Begünstigung durch Aufwendungszuschüsse ist bei einer steuersystematisch gebotenen realitätsgerechten Erfassung des Mietwerts der eigengenutzten Wohnung unproblematisch, da die abzugsfähigen Aufwendungen im Umfang der Subventionierung sinken, somit der steuerpflichtige Teil des Mietwerts im gleichen Ausmaß steigt. Bei Vernachlässigung des Mietwerts der eigengenutzten Wohnung (wie derzeit) bietet sich behelfsweise eine Kürzung des Grundfreibetrags um den Subventionsbetrag an, da die dort berücksichtigten Aufwendungen für Wohnen im Umfang der Subvention niedriger anzusetzen sind. Systematisch ist diese Vorgehensweise allein deshalb nicht voll zufriedenstellend, weil im Existenzminimum nur ein Grundbedarf an Wohnungsaufwendungen berücksichtigt ist, insofern eine Obergrenze des Abzugs besteht, während die genannten Subventionen sich auf einen gehobenen Bedarf beziehen, also darüber hinausgehen können. Eine Kürzung des Grundfreibetragsanteils für Wohnzwecke ist 
im vollen Umfang angebracht, allerdings dürfte in einer Anzahl von Fällen die Nichtnegativitätsbedingung greifen.

Die Förderung des Erwerbs von Wohneigentum durch Abschreibungsvorteile ist höchst intransparent und tendenziell mit regressiven Verteilungswirkungen verbunden. Prinzipiell ist eine einkommensteuerliche Erfassung von Steuersubventionen denkbar (nach Festlegung einer Steuernorm), die derjenigen von Kreditsubventionen entspricht. Vorzuziehen ist jedoch deren Ersetzung durch einmalige einheitliche Einmalprämien im Erwerbs- bzw. Fertigstellungszeitpunkt. Eine solche in erster Linie objektbezogene Förderung könnte dann steuerfrei bleiben.

\section{BAföG-Leistungen 13}

\subsection{Charakterisierung 14}

Ziel des Bundesausbildungsförderungsgesetzes ist es, eine der individuellen Neigung, Eignung und Leistung entsprechende Ausbildung finanziell zu ermöglichen (§ 1 BAföG).

Zur Berechnung der individuellen Förderung werden nach Ausbildungsart und Art der Unterbringung differenzierte Bedarfssätze zugrundegelegt, die die Kosten des Lebensunterhalts und der Ausbildung abdecken sollen. Diese pauschalen Bedarfssätze werden bei besonders hohen Mietbelastungen, Kosten für Krankenversicherung und Auslandsaufenthalte aufgestockt. Der tatsächliche Förderungsbetrag ergibt sich durch Kürzung des so ermittelten Bedarfs um anzurechnende Einkommen des Antragstellers, seines Ehegatten und seiner Eltern. Der Förderungsbetrag wird für Schüler als Zuschuß, für Studenten i.d.R. je zur Hälfte als Zuschuß und als Darlehen geleistet.

13 Die hier vorgetragenen Überlegungen lassen sich auf alle Arten von Stipendien u.ä. übertragen.

14 Vgl. zu weiteren Einzelheiten das Bundesausbildungsförderungsgesetz sowie Bundesminister für Bildung und Wissenschaft (1992). 
Sofern der Auszubildende über eigenes Vermögen oberhalb eines Freibetrags verfügt, sein Ehegatte oder seine Eltern vermögensteuerpflichtig sind, wird eine Förderung nicht bewilligt.

Die im Rahmen des BAföG geleisteten Darlehen bleiben unverzinst, die Rückzahlungsbedingungen werden an die Zahlungsfähigkeit des Geförderten angepaßt. Teile des Darlehensbetrags werden bei Kindererziehung, bei besonders erfolgreichem und schnellem Abschluß der Ausbildung sowie bei vorzeitiger Rückzahlung erlassen.

Die Leistungen nach dem BAföG werden im Rahmen der steuerpflichtigen Einkünfte nicht erfaßt, so daß auch weitere Einkünfte, insbesondere bis zur Anrechnungsgrenze des BAföG, steuerfrei bleiben.

\subsection{Einkommensteuersystematische Aspekte}

Sofern die Leistungen nach dem BAföG als Zuschuß gewährt werden, erhöhen sie die Leistungsfähigkeit des Empfängers und sollten daher in die Bemessungsgrundlage der Einkommensteuer einfließen. Aufgrund der Zielsetzung und Höhe dieser Leistungen ist allerdings davon auszugehen, daß sie wegen des Existenzminimum-Grundfreibetrags der Einkommensteuer faktisch steuerfrei bleiben. Erst darüber hinausgehende weitere Einkünfte würden zu einer tatsächlichen Steuerpflicht führen, was auch voll gerechtfertigt erscheint 15 . Das gleiche gilt für alle Arten von Stipendien.

Sofern es sich bei den Leistungen um (zinslose) Darlehen handelt, könnte zunächst daran gedacht werden, diese Zahlungen wie bei Konsumentenkrediten üblich als einkommensteuerlich irrelevant zu betrachten. Der jährliche Zinsvorteil könnte allerdings als Transfer erfaßt werden. Zur Bewertung kommen die gleichen Verfahren wie für "fringe

15 In den BAföG-Leistungen berücksichtigten Aufwendungen für Lehrmittel, die über die normalen Lebenshaltungskosten hinausgehen, sollte innerhalb der Einkommensteuer ein entsprechender Sonderfreibetrag Rechnung tragen (vgl. § 10(1) Nr.7 EStG). Zusätzliche Einkünfte in geringem Umfang blieben zudem aufgrund von Werbungskostenpauschbeträgen steuerfrei. 
benefits" in Betracht (OECD, 1988a, 17f.). Für Kredite im Rahmen des BAföG ist eine solche Vorgehensweise jedoch wenig empfehlenswert:

- Sie haben die laufende Sicherung des Existenzminimums der Auszubildenden zum Ziel, womit die oben skizzierte steuerliche Behandlung nicht korrespondiert. Den Auszubildenden würde - sofern sie nicht über andere Einkünfte verfügen - der Existenzminimum-Grundfreibetrag in der Ausbildungsphase faktisch versagt, die Darlehensrückzahlung müßte aus versteuertem Einkommen erfolgen, was damit zu einer Besteuerung des Existenzminimums führen würde.

- Werden Darlehensbeträge erlassen, wie das derzeit unter bestimmten Umständen vorgesehen ist, wären diese Beträge nachträglich als Einkommen zu verbuchen.

Sehr viel einfacher und im Hinblick auf die Zielsetzung des BAföG (Finanzierung des laufenden Lebensunterhalts) adäquater erscheint es daher, im Rahmen einer Sonderregelung hier auch die Darlehensgewährung als Transfereinkommen zum Zeitpunkt des Zuflusses zu verbuchen, die Rückzahlung von BAföG-Darlehen in ihrer tatsächlichen Höhe zum Abzug von der Einkommensteuer-Bemessungsgrundlage zuzulassen. Eine solche Vorgehensweise hat den weiteren Vorteil, daß für Zwecke der einkommensteuerlichen Behandlung nicht zwischen Darlehen und Zuschüssen unterschieden werden muß, da beide generell die Steuerbemessungsgrundlage erhöhen, während allein die Rückzahlung von Darlehen die Steuerbemessungsgrundlage reduziert 16 .

Ein weiterer Aspekt der Darlehensgewährung ist der Zinsvorteil, der ebenfalls als Einkommen des Darlehensnehmers gelten kann. Eine laufende einkommensteuerliche Erfassung ist jedoch ausgeschlossen, da den Auszubildenden im Regelfall keine zusätzlichen Mittel für eine Steuerzahlung zur Verfügung stehen; bei einer summarischen Erfassung des Zinsvorteils nach Abschluß der Ausbildung müßte der genaue Zeitpunkt

16 Als "Problemfall" verbleiben dann lediglich noch private Kredite, die der Ausbildungsfinanzierung dienen. Solche Fälle dürften allerdings in der Ausbildungsfinanzierung wegen der in aller Regel vorliegenden Unterhaltsoder BAföG-Ansprüche sowie der mangelnden Bonität von Auszubildenden nahezu ausgeschlossen sein. 
einer steuerlichen Erfassung bestimmt werden. Angesichts der Modalitäten der BAföG-Darlehen, die aufgrund von Teilerlassen wenig mit üblichen Krediten gemein haben und als Alternative zur vollständigen Kostenübernahme in Form von Zuschüssen zu sehen sind, ist eine aus prinzipiellen Überlegungen heraus durchaus naheliegende steuerliche Erfassung des Zinsvorteils kein überzeugender Beitrag im Rahmen der hier skizzierten steuerlichen Gesamtlösung für diesen Teilbereich.

\section{Kindergeld}

\subsection{Charakterisierung}

Die aufgrund des Bundeskindergeldgesetzes gewährten Leistungen sollen einen Teil der kindesbedingten Belastungen der Eltern ausgleichen und die Startchancengleichheit der Kinder verbessern.

Anspruchsberechtigt für sich selbst oder ihre Kinder sind alle Personen, die (im Regelfall) in Deutschland ihren Wohnsitz oder gewöhnlichen Aufenthalt haben. Berücksichtigungsfähige Kinder im Sinne des $\S 2$ BKGG sind generell Kinder bis zum 16. Lebensjahr, darüber hinaus insbesondere Kinder in Schul- oder Berufsausbildung bis maximal zum 27. Lebensjahr. Kindergeld wird nur gewährt, sofern kein Anspruch auf dem Kindergeld vergleichbare Leistungen besteht.

Die Höhe des Kindergeldes ist nach der Kinderzahl gestaffelt und beträgt $70 \mathrm{DM}$ für das erste, $130 \mathrm{DM}$ für das zweite, $220 \mathrm{DM}$ für das dritte und 240 DM für das vierte und jedes weitere Kind. Bei Überschreiten bestimmter Einkommensgrenzen wird das Kindergeld für zweite und weitere Kinder stufenweise bis auf Sockelbeträge von $70 \mathrm{DM}$ für das zweite und 140 DM für jedes weitere Kind reduziert ( 10 BKGG). Für Kindergeldberechtigte mit geringen Einkommen, die infolgedessen die Kinderfreibeträge des Einkommensteuergesetzes nicht oder nicht voll nutzen können, wird ein Zuschlag zum Kindergeld geleistet, der pro Jahr maximal 19\% der Summe der dem Berechtigten zustehenden Kinderfreibeträge beträgt ( $\S 11 \mathrm{a}$ BKGG) und damit der Steuerentlastung durch 
Kinderfreibeträge in der Eingangsproportionalzone des Einkommensteuertarifs entspricht. Das Kindergeld ist nach $\S 3 \mathrm{Nr} .24$ EStG explizit von der Einkommensteuer befreit.

\subsection{Einkommensteuersystematische Aspekte}

Das Kindergeld ist neben den Kinderfreibeträgen der Einkommensteuer das wichtigste Instrument des Kinderlastenausgleichs. Ziel ist es, gemeinsam 17 mit dem Kinderfreibetrag die Belastungen durch Kinder adäquat aufzufangen. Eine einkommensteuerliche Behandlung, die diese Interdependenz außer acht ließe, würde analog zur Behandlung anderer direkter Transfers eine Steuerpflicht des Kindergeldes bzw. eine Anrechnung des Kindergeldes auf den sonst zu gewährenden Kinderfreibetrag fordern, da dieses für sich betrachtet die Leistungsfähigkeit des Empfängers erhöht (Lang, 1990, 339f.).

Wegen der gemeinsamen Zielsetzung von Kindergeld und Kinderfreibetrag, die dazu fürt, daß auch im politischen Entscheidungsprozeß beide Instrumente als interdependent betrachtet werden, wird hier für eine umfassende Erörterung der Problematik auf das Kapitel "Allgemeiner Kinderlastenausgleich" verwiesen.

17 Vgl. hierzu die jüngsten Entscheidungen des Bundesverfassungsgerichts (1991a; 1991b). 


\section{VERSICHERUNGSTRANSFERS}

\section{Alterssicherung}

\subsection{Charakterisierung}

Heute findet sich die Alterssicherung ganz selbstverständlich im Zielkatalog sozialer Sicherung: Die Meinung, daß ab einem bestimmten Alter die Erzielung von Arbeitseinkommen nicht mehr möglich oder jedenfalls nicht mehr zumutbar ist, stößt auf allgemeine Zustimmung1. Daraus ergibt sich die Notwendigkeit, für die Zeiten altersbedingter NichtErwerbstätigkeit finanziell vorzusorgen und damit andere Quellen für den Lebensunterhalt zu erschließen (abgesehen von der traditionellen Absicherung innerhalb der Großfamilie, wo nicht finanziell, sondern in Form von Kindern Altersvorsorge getroffen wurde) ${ }^{2}$. Ziel der Altersvorsorge ist es damit, einen ausreichenden und gleichmäßigen Zufluß finanzieller Mittel über die gesamte verbleibende Lebenszeit hinweg sicherzustellen. Um den während der Aktivenzeit erreichten Lebensstandard zu halten, wird im allgemeinen ein ähnlich hohes Nettoeinkommen erforderlich sein. Lediglich der Wegfall berufsbedingter Aufwendungen spricht für einen etwas geringeren Bedarf. Sofern die Mobilität alter Menschen abnimmt, dürfte das vor allem eine Änderung der Ausgabenstrukturen mit sich bringen (Wegfall von Ausgaben für Freizeitaktivitäten einerseits, zusätzliche Aufwendungen für Fahrtkosten, Haushaltshilfen u.ä. andererseits) ${ }^{3}$. Unbestritten ist dagegen ein erhöhter

$1 \mathrm{Vgl}$. Bundesminister für Arbeit und Sozialordnung (1990, 41-49) und Lampert (1991, 219-240). Noch vorsichtig abwägend SozialenquêteKommission (1966, 69, Ziff. 169).

2 Zur Integration von Kinderleistungen in ein finanzielles Alterssicherungssystem vgl. beispielsweise Nell-Breuning (1978), Schmidt/Frank/MüllerRohr (1985), Erbe (1986, 200).

3 Vgl. Littmann (1983, 434f.). Achinger u.a. $(1955,103)$ gehen von einem erheblich geringeren Bedarf aus. Der Wissenschaftliche Beirat beim BMF (1986, 544) spricht sich dagegen für die Berücksichtigung eines Mehrbedarfs aus, allerdings wohl vor allem, um besondere Härten bei einem plötzlichen Übergang zu einer Rentenbesteuerung zu vermeiden. Der in der 
Bedarf in Fällen von Pflegebedürftigkeit, die in weiter Abgrenzung für ca. 4-5\%, in enger Abgrenzung (bettlägerig) für ca. $1 \%$ der über 60 jährigen gegeben ist ${ }^{4}$.

Gemessen jeweils an der Bevölkerungsgruppe der 55-65-jährigen ist die gesetzliche Rentenversicherung mit Abstand das bedeutendste Alterssicherungssystem, aus dem $92 \%$ der Männer und $79 \%$ der Frauen eine eigene Rente beziehen oder erwarten. Unter Einbeziehung des Anspruches auf Hinterbliebenenleistungen, die aufgrund der geschlechtsspezifischen Lebenserwartung und des Altersunterschieds zum Ehepartner besonders für Frauen relevant sind, schließt die gesetzliche Rentenversicherung 98\% der Frauen in ihr Sicherungssystem ein. Daneben existiert als weiteres Regelsicherungssystem die Beamtenversorgung, aus der unter Anrechnung eventueller Bezüge aus der gesetzlichen Rentenversicherung - $12 \%$ der Männer und 2\% der Frauen eine eigene Pension beziehen oder erwarten.

Als Zusatzsicherungssysteme sind für Männer einerseits, Frauen andererseits vor allem die betriebliche Altersversorgung für $25 \%$ bzw. 7\%, die Zusatzversorgung des öffentlichen Dienstes für $11 \%$ bzw. $8 \%$ sowie die landwirtschaftliche Altershilfe für $7 \%$ bzw. $1 \%$ von Bedeutung (die Zahlen beziehen sich nur auf originäre Ansprüche) ${ }^{5}$.

Aufgrund der vorhandenen Leistungskumulationen von Alterssicherungsleistungen aus den genannten Sicherungssystemen und weiteren vor allem individuell gestalteten Alterssicherungsmaßnahmen lassen sich

Sozialhilfe vorgesehene Mehrbedarf kann hier kaum als Referenzgröße dienen, da er offensichtlich der Diskriminierung erwerbsfähiger, jedoch nicht erwerbstätiger, Sozialhilfeempfänger dient und somit durch seine Berücksichtigung erst ein "Normbedarf" erreicht wird (vgl. § 23 BSHG sowie Kapitel II.2.8).

4 Zahlenangaben zur Hilfebedürftigkeit und Pflegebedürftigkeit alter Menschen finden sich in Infratest Sozialforschung (1990, Bd.I, 63-70).

5 Alle quantitativen Angaben zum Umfang der Alterssicherung sind der Studie von Infratest Sozialforschung (1990, Bd. Z, 12-14, 32f.) entnommen. 
über die Größenordnung von im Einzelfall zufließenden "Alterseinkünften" nur sehr eingeschränkt Aussagen machen6.

\subsection{Einkommensteuersystematische Aspekte}

\subsubsection{Steuerfreiheit des Existenzminimums im Alter}

Die Forderung nach Steuerfreiheit des persönlichen Existenzminimums ${ }^{7}$ läßt sich auch auf Aufwendungen zur Sicherung des Existenzminimums im Alter übertragen. Im Alter besteht zwar formal weiterhin die Möglichkeit, den Grundfreibetrag der Einkommensteuer, der dem Existenzminimum entsprechen sollte ${ }^{8}$, in Anspruch zu nehmen; sofern aber der Lebensunterhalt aus der Auflösung von Vermögen bestritten wird, im Extremfall also ein Einkommen der entsprechenden Periode von Null festzustellen ist, greift diese Regelung faktisch nicht ${ }^{9}$ : auch die Aufwendungen für das Existenzminimum sind dann aus - allerdings in vorangegangenen Perioden - versteuertem Einkommen zu bestreiten.

6 So werden insbesondere für die Alterssicherung bestimmte Kapitalansammlungen, z.B. in der Form von Kapital-Lebensversicherungen, nicht erfaßt, und auch die Erfassung von Kapitaleinkünften ist lückenhaft. Die oben genannte Studie von Infratest Sozialforschung ermittelt in einer umfassenden Befragung für das Jahr 1986 für die Bevölkerungsgruppe der Ruheständler und über 65-jährigen ein durchschnittliches monatliches Nettoeinkommen in Höhe von 2243 DM für Männer und 1271 DM für Frauen. Auf der Haushaltsebene betrug das durchschnittliche monatliche Nettoeinkommen bei Differenzierung nach der letzten beruflichen Tätigkeit der Bezugsperson für Arbeiter, Angestellte und Beamte zwischen 950 DM (Haushalte von ledigen oder geschiedenen angelernten Arbeiterinnen) und 4967 DM (Haushalte von Ehepaaren mit Bezugsperson als Beamter im höheren Dienst).

7 Vgl. Kapitel II.2.8.

8 Gegebenenfalls ist er bei Vorliegen außergewöhnlicher Belastungen aufzustocken.

9 Es handelt sich insbesondere deshalb um einen Extremfall, weil aus vorhandenem Vermögen i.d.R. mit Zinseinkünften oder anderen Vermögenseinkünften zu rechnen ist. 
Neben umfassenden Systemen der Lebenseinkommensbesteuerung 10 bieten sich grundsätzlich zwei Lösungsmöglichkeiten an, um eine solche Besteuerung des Existenzminimums zu vermeiden:

Zum einen könnte eine Steuerrückerstattung der bereits auf das materielle Existenzminimum gezahlten Einkommensteuer gewährt werden. Ausschlaggebend sollte hierfür allerdings der jeweilige persönliche marginale Steuersatz der Vorsorgeperiode sein, was - selbst wenn die Zuordnung auf einzelne Vorsorgeperioden gelingen würde - außerordentlich aufwendig und intransparent für den Steuerzahler ist.

Zum anderen könnte bereits zum Zeitpunkt der Altersvorsorge ein Vorsorgebetrag, der lediglich der Sicherung des Existenzminimums im Alter diente, von der Einkommensteuer befreit werden. Die Abgrenzung und Kontrolle des Sachverhalts Altersvorsorge müßte in diesem Fall allerdings ausreichend geklärt werden. Zwar kommt prinzipiell eine große Zahl von Vorsorgeformen in Frage, eine Sicherung der Zweckbestimmung und eine ungefähre Abschätzung des Vorsorgebedarfs bei unterschiedlichen individuellen Lebenserwartungen ist aber allein bei einer Leibrentenversicherung möglich. Unter bestimmten vereinfachenden Annahmen läßt sich so ein Vorsorgebedarf für eine jährliche Rentenzahlung ab dem 65. Lebensjahr von 10.000 DM in heutigen Werten von ca. 3.400 DM pro Jahr (40 Beitragsjahre) oder in Form einer Kapitalsumme in Höhe von ca. 160.000 DM ermitteln ${ }^{11}$. Bei der Steuerbefreiung

10 Vgl. Kapitel II.2.5.

11 Die Berechnung erfolgt unter der Voraussetzung, daß Kalkulationszinssatz, Steigerungssatz der Beiträge und Steigerungssatz des (Sozialhilfe-) Existenzminimums einander entsprechen, wodurch die Berücksichtigung einer Zinskomponente entfallen kann. Eine Beitragszahlung wurde zwischen dem 25. und 65. Lebensjahr angenommen, die Auszahlungen setzen mit dem 65. Lebensjahr ein. Die durchschnittliche Lebenserwartung eines bzw. einer 65-jährigen wurde dabei mit 15,93 Jahren als ungewichtetes arithmetisches Mittel der geschlechtsspezifischen Lebenserwartungen angesetzt; der Ausfall von Ansprüchen durch Versterben vor dem 65. Lebensjahr wurde durch die Sterbewahrscheinlichkeit zwischen dem 45. und 65. Lebensjahr angenähert (zu den bevölkerungsstatistischen Grunddaten vgl. Statistisches Bundesamt, 1991, 82f.). Dementsprechend ergibt sich als Beitrag pro Jahr 10.000 DM x 15,93 x 0,8541: $40=3401,45$ DM, als notwendige Kapitalsumme 10.000 DM x 15,93 $=159.300$ DM. 
einer solchen Vorsorge für das Existenzminimum im Alter ist dann allerdings sicherzustellen, daß im Alter ein Existenzminimum-Grundfreibetrag nicht nochmals (zusätzlich) in Anspruch genommen werden kann. Sofern das Alters-Existenzminimum jedoch aus der Auflösung anderen Vermögens bestritten würde, müßte in solchen Ausnahmefällen doch eine Rückerstattung von Steuern wie oben beschrieben stattfinden, diese sollte aus Vereinfachungsgründen zu einem einheitlichen Steuersatz erfolgen 12 . Außer der im Einzelfall willkürlichen Festlegung des Steuersatzes bleibt hier nur kritisch anzumerken, daß in diesen Fällen der Steuerrückerstattung ein steuerlicher Anreiz zur Vorsorge erst im Alter und nicht bereits in der Aktivenphase spürbar würde, womit kaum Verhaltensänderungen im gewünschten Sinn induziert werden könnten, wenn auch eine finanzielle Entlastung gerade in der Altersphase benötigt wird.

\subsubsection{Lebenseinkommensbesteuerung}

Während Vermögenseinkommen grundsätzlich unabhängig von der Möglichkeit des persönlichen Arbeitseinsatzes ist, daher - bei gegebenem Vermögensbestand - auch im Alter weiterfließt, ist dies bei Arbeitseinkommen anders. Daher scheint es angebracht, aus der Sicht einer Lebenseinkommensbesteuerung bei Arbeitseinkommen Maßnahmen zu fordern, die eine gleichmäßige, die sonst zu starke Progression des Einkommensteuertarifs dämpfende, Verteilung über die gesamte Lebenszeit der Steuerpflichtigen ermöglichen (so bereits Mill, 1902, 489f.). Für Vermögenseinkommen ist dies jedenfalls bei konstantem realem Vermögensbestand und auch sonst konstanten Rahmenbedingungen nicht erforderlich. Für einen solchen interperiodischen Progressionsausgleich bieten

12 Dieser Steuersatz sollte in der Nähe des Steuersatzes der Eingangsproportionalzone des Einkommensteuertarifs liegen, da i.d.R. von einer gewissen Korrelation zwischen Einkommens- und Vermögenshöhe ausgegangen werden kann, Vermögen in den hier zu betrachtenden Erstattungsfällen (keine nennenswerten Einkünfte aus Vermögen!) aber offensichtlich nur in relativ geringem Umfang vorliegt. Bei dieser Pauschalierung wäre grundsätzlich auch an eventuelle Tarifänderungen und eine Zinskomponente zu denken. 
sich zunächst explizite Verfahren einer Lebenseinkommensbesteuerung an ${ }^{13}$.

Sofern diese Verfahren jedoch auf kürzere Zeiträume hin angelegt sind, wie z.B. das unter Praktikabilitätsaspekten vorzuziehende "Block Averaging", sind sie nicht in der Lage, langfristig auftretenden Einkommensunterschieden wie zwischen Erwerbs- und Ruhestandsperiode Rechnung zu tragen. In bezug auf die Besteuerung der Alterssicherung bieten sich dann als Ergänzung vereinfachende Verfahren an, die Vorsorgeaufwendungen bis zur Absicherung des laufenden Arbeitseinkommens (abzüglich der Vorsorgeaufwendungen selbst) steuerfrei lassen, dafür jedoch den späteren Zufluß im Alter steuerlich belasten (Haller, 1973, 526; Hackmann, 1979, 106-109). Damit wird ein Progressionsausgleich erreicht, in Kauf genommen werden muß dann allerdings ein durch die Verschiebung der Steuerbemessungsgrundlage hervorgerufener Zinsvorteil der Steuerpflichtigen.

Die Bestimmung der Höhe des Arbeitseinkommens wirft jedoch innerhalb eines solchen Ansatzes insbesondere bei Selbständigen und Gewerbetreibenden Probleme auf, die nur durch Pauschalierungen lösbar scheinen. Hinzu kommen Fragen der Abgrenzung von Altersvorsorgeaufwendungen und der Ermittlung ihrer adäquaten Höhe, welche wiederum nur in der Form der Leibrentenversicherung zu einer kalkulierbaren Größe wird. Auch ist es allein die Leibrentenversicherung, die im Alter gleichmäßige Zuflüsse bis zum Lebensende garantiert und insofern auch in dieser Phase ohne Anwendung weiterer glättender Verfahren den Ansatzpunkt für die Verwirklichung einer solchen vereinfachten Form einer Lebenseinkommensbesteuerung liefert.

Sofern das Etikett "Vorsorgeaufwendung" eine Verschiebung von einkommensteuerlich unbelasteten Einkommensteilen in die Zukunft und damit einen Zinsvorteil oder gar völlige Steuerfreiheit ermöglicht, ist auch eine mißbräuchliche Deklarierung von Beträgen, die der allgemeinen Vermögensbildung, gegebenenfalls auch über den Tod hinaus, dienen sollen, zu erwarten. Tendenziell wirkt einem solchen Mißbrauch eine

13 Vgl. Kapitel II.2.5. 
verstärkte Steuerprogression im Alter entgegen, falls diese Beträge dann erfaßt werden und aufgrund überhöhter Vorsorge das steuerpflichtige Einkommen dasjenige der Aktivenphase übersteigt. Hinzu kommt eine i.d.R. anzutreffende Gegenwartspräferenz der Steuerpflichtigen. Ausgeschlossen wird ein solcher steuerlicher Anreiz erst dann, wenn dessen Ursache, nämlich der Zinsvorteil, beseitigt wird. Dies wäre durch eine im Interesse einer idealen Lebenseinkommensbesteuerung liegenden Verzinsung der auf den Betrag der Vorsorgeaufwendungen entfallenden fiktiven Steuerschuld realisierbar (Vickrey, 1959), allerdings sehr aufwendig in der Durchführung. Eine Reduzierung möglichen Mißbrauchs wird auch durch eine Einschränkung der Wahlfreiheit der Vorsorgeform erreicht. Dadurch können andere Sparmotive als das der reinen Vorsorge für den Lebensbedarf im Alter weitgehend ausgeschlossen werden 14 .

\subsubsection{Alterssicherung als meritorisches Gut}

Eigenvorsorge für das Alter ist gesellschaftlich erwünscht. Hierfür spricht allein schon die Orientierung am Subsidiaritätsprinzip, wonach möglichst große Regelungsbereiche in der Eigenverantwortung der Bürger bleiben sollten. Nach einer anderen Argumentation weist Altersvorsorge Eigenschaften eines (staatlich zu finanzierenden) öffentlichen Gutes auf (Spahn/Kaiser, 1988, 201-208): Sie beinhaltet positive externe Effekte für die Gesellschaft insgesamt, insbesondere durch eine Förderung des sozialen Friedens, einer gleichmäßigen Einkommensverteilung und der Eigenverantwortung der Bürger. Insofern ist zu überlegen, ob im Sinne

14 Das Vorsorgemotiv kann nur für Leibrentenversicherungen in reiner Form vorausgesetzt werden, da nur diese einen gleichmäßigen Einkünftezufluß über die gesamte Lebensdauer hinweg absichern, was oben exakt als Vorsorgeziel definiert wurde. Bei allen anderen Vorsorgeformen können daher zusätzliche Motive unterstellt werden, die sich letztlich aus der Verfügbarkeit über das Vorsorgevermögen erklären lassen und von unterschiedlichem Gewicht sind (z.B. Vererbbarkeit, jederzeitige Verfügbarkeit für andere Zwecke als die Sicherung des Lebensunterhalts im Alter, wenn auch möglicherweise in zeitlich oder sachlich beschränkter Form). Diese Motive können unter Umständen sogar völlig dominieren. 
eines meritorischen Gutes ${ }^{15}$ Altersvorsorge staatlich gefördert werden sollte. Ihr "Öffentlicher-Guts-Anteil" ist aber letztlich auch nicht annähernd bestimmbar, und somit läßt sich die Frage der Höhe einer möglichen staatlichen Förderprämie nach allgemein akzeptablen Kriterien nicht beantworten. Die Argumentation bleibt zu vage, als daß eine Förderung jeder Form von Altersvorsorge und damit auch jeder Form der Vermögensbildung vertretbar wäre 16 .

Für den Bereich der Mindestsicherung gibt es allerdings ein besonderes öffentliches Interesse an ausreichender privater Vorsorge, da Bürger ohne ausreichende Vorsorge über die Inanspruchnahme von Sozialhilfe-Zahlungen in vollem Umfang der Gesellschaft zur Last fallen ${ }^{17}$. Sofern eigene Vorsorge in Höhe des Existenzminimums getroffen wurde, bedeutet die staatliche Absicherung über die Sozialhilfe gleichzeitig eine Diskriminierung dieser Vorsorgeaktivitäten ${ }^{18}$. Durch steuerliche Maßnahmen sollte diese Diskriminierung nicht noch verstärkt werden, so daß auch aus dieser Sicht die Steuerfreiheit des Existenzminimums im Alter dringend geboten ist. Die Frage, ob eine steuerliche Nicht-Diskriminierung ausreichend oder darüber hinaus eine Förderung der Mindestsicherung lediglich der Bürger mit niedrigem Einkommen oder aber aller Bürger vorzusehen ist, wird wohl umstritten bleiben, zumal auch das oben dargestellte Öffentliche-Guts-Argument ganz besonders für den Bereich der Mindestsicherung angeführt werden kann ${ }^{19}$. Als Alternative zu einer Förderung besteht allerdings die Möglichkeit einer

15 Für die klassische Definition vgl. Head (1966).

16 Die Sachlage weist Parallelen zu den sogenannten pekuniären externen Effekten auf, die ebenfalls die Folge allgemeiner Interdependenzen des betrachteten Systems sind (Schlieper, 1980, 524). Eine eher distanzierte Haltung zur Altersvorsorge als öffentlichem Gut nimmt auch Krupp (1988, 504f.) ein.

17 So bereits Lampe $(1928,189)$.

18 Eine solche Diskriminierung läßt sich allerdings rechtfertigen, sofern gesichert ist, daß die Sozialhilfegewährung auf Ausnahmefälle beschränkt bleibt.

19 Dem entspricht wohl auch - losgelöst von der Altersvorsorgediskussion die steuerliche Freistellung von Vermögen und Zinseinkünften in gewissem Umfang innerhalb der Vermögen- bzw. Einkommensteuer. 
Mindest-Vorsorgepflicht für alle Bürger, wodurch alles getan wird, die Sozialhilfegewährung im Alter auf jene Bürger zu beschränken, die nie zu einer ausreichenden individuellen Vorsorge in der Lage waren. Unter meritorischen Aspekten kann dann eine Förderung entfallen.

\subsubsection{Zwang zur Alterssicherung}

Durch den Zwang zur Vorsorge, insbesondere durch gesetzlichen Zwang wie er i.d.R. für Arbeiter und Angestellte besteht, wird das für gegenwärtige Bedürfnisbefriedigung disponible Einkommen reduziert. In der Literatur wird oft bereits aus diesem Umstand auf eine im gleichen Umfang geminderte steuerliche Leistungsfähigkeit geschlossen und daher der Abzug der Aufwendungen zur Zwangsvorsorge von der Bemessungsgrundlage der Einkommensteuer gefordert ${ }^{20}$. Da mit den Altersvorsorgeaufwendungen aber Ansprüche erworben (evtl. auch Vermögenswerte geschaffen) werden, ist dieser Sachverhalt nur schwer mit anderen leistungsfähigkeitsmindernden Aufwendungen - bei denen dies nicht der Fall ist - vergleichbar. Als adäquate Lösung, die einer gegenwärtigen Reduktion des - auch für Steuerzwecke - verfügbaren Einkommens Rechnung tragen würde, wäre jedenfalls auch eine Stundung der Steuerschuld unter Inrechnungstellung von Zinsen denkbar. Eine darüber hinausgehende Berücksichtigung individueller Zusatzlasten (Excess burden) einer Zwangsversicherung ist allerdings kaum möglich ${ }^{21}$.

Unter dem Zwangsaspekt zu unterscheiden sind weiterhin Fälle, in denen tatsächlich ein gesetzlicher Zwang vorliegt ${ }^{22}$ und solche, in denen allenfalls ein faktischer Zwang konstatiert werden kann ${ }^{23}$. Zu beachten ist, daß in den Fällen der zuletzt genannten Art erheblich größere persönliche Spielräume hinsichtlich Art und Umfang der Altersvorsorge bestehen. Damit es nicht zu einer horizontalen Ungleichbehandlung von

20 Vgl. Kapitel III.3.2.

21 Vgl. Kapitel II.2.2 und III.3.2.

22 Hierzu sind auch Fälle von Quasi-Zwang zu rechnen - vgl. dazu auch Kap. III.3.2.

23 Vgl. beispielsweise Söhn (1985, 404: "Zwangsaufwendungen i.w.S.") sowie Steuerreformkommission (1971, 127, Rz. 251f.). 
Steuerpflichtigen kommt, dürfte daher bei einer steuerlichen Sonderbehandlung für Zwangsbeiträge bereits die Öffnung der Zwangsversicherung für alle Bürger zu den dort üblichen Konditionen bei steuerlicher Gleichstellung der Beiträge genügen.

\subsubsection{Schlußfolgerungen}

Zusammenfassend läßt sich festhalten, daß eine Absicherung des Existenzminimums im Alter aus steuerfreiem Einkommen ermöglicht werden sollte. Dies folgt bereits aus der Forderung nach steuerlicher Nichtdiskriminierung individueller Vorsorgeaktivitäten sowie nach Steuerfreiheit des Existenzminimums. Ergänzend bietet sich die Regelung einer Vorsorgepflicht in dieser Höhe an, womit auch den meritorischen Aspekten Rechnung getragen wird 24 . Die Forderung nach steuerlicher Freistellung jeglicher Vermögensbildung, da sie unter anderem auch dem Zweck der Altersvorsorge dienen kann, ist dagegen überzogen.

\subsection{Sicherungsarten}

Bevor detaillierter auf die einzelnen möglichen Formen der Altersvorsorge und deren einkommensteuerliche Behandlung eingegangen wird, kann hier schon festgehalten werden, daß bei den einzelnen Vorsorgeformen in unterschiedlichem Ausmaß der Aspekt der reinen Altersvorsorge im Mittelpunkt steht und sich auch Anknüpfungspunkte für die gerade dargestellten einkommensteuersystematischen Überlegungen in unterschiedlicher Weise ergeben.

Sofern es eine Form der Altersvorsorge gibt, die ausschließlich dem Motiv der Alterssicherung folgt, ist es für den Steuergesetzgeber naheliegend, sich dieser Vorsorgeform als Referenzsystem zu bedienen, unter Umständen sogar mögliche steuerliche Vorteile auf diese Form zu beschränken, womit dann allerdings auch die individuelle Wahl der

24 Hierzu geeignet ist der Vorschlag von Krupp (1987, 9-13), nach dem alle Bürger einen eigenständigen Anspruch auf soziale Sicherung im Alter erwerben. 
Vorsorgeform beeinflußt würde. Eine solche Sonderstellung nimmt die Leibrentenversicherung ein, weil sie ausschließlich der Alterssicherung dient und sich auf die gesamte Lebenszeit erstreckt. Sie sichert - bei insgesamt ausreichender Vorsorgehöhe - das Existenzminimum über die gesamte Lebenszeit. Auch sind allein hier die notwendigen Aufwendungen recht gut kalkulierbar. Bei allen anderen Formen der Alterssicherung sind andere Motive zumindest mit beteiligt, was sich aus der Verfügbarkeit der aufgewendeten Beträge für andere Zwecke sowie aus dem Fehlen eines Zuschnitts der Sicherung auf die gesamte Restlebenszeit ergibt.

\subsubsection{Allgemeine Vermögensbildung}

Als allgemeine Vermögensbildung werden hier alle Arten der Vermögensbildung erfaßt, sofern sie nicht durch besondere Ausgestaltung auf Zwecke der Altersversorgung ausgerichtet sind (bzw. ausgerichtet zu sein vorgeben) und daher später noch gesondert behandelt werden 25 . Insbesondere auch das Kontensparen, der Erwerb von Wertpapieren sowie von Immobilien fallen in den hier zu behandelnden Bereich.

Vermögensbildung ist grundsätzlich dazu geeignet, zur Vorsorge für das Alter zu dienen. Eine Steuerfreiheit der Vermögensbildung verbunden mit einer Steuerpflicht von Vermögensauflösungen (also eine persönliche allgemeine Verbrauchsteuer) würde zudem tendenziell einer Lebenseinkommensteuer nahekommen (Kaldor, 1955, 52).

Eine Verwendung des Vermögens für Zwecke der Sicherung des Lebensunterhalts im Alter ist jedoch nicht gewährleistet, die dazu notwendige Summe durch die Abhängigkeit von der individuellen Lebenserwartung der Steuerpflichtigen zudem nicht ex ante bestimmbar. Die großzügige Steuerbefreiung der Ersparnisbildung würde eine starke Annäherung an eine persönliche allgemeine Verbrauchsteuer bedeuten, die auf einer anderen Auffassung von steuerlicher Leistungsfähigkeit

25 Für die Abgrenzung war weniger die besondere Eignung für Zwecke der Altersversorgung, die später noch im einzelnen zu untersuchen ist, als vielmehr die traditionelle Zuordnung $\mathrm{zu}$ den Vorsorgeaufwendungen ausschlaggebend, wie sie sich auch im derzeitigen Einkommensteuerrecht findet. 
beruht 26 . Auch Argumente einer steuerlichen Förderung der Eigenvorsorge rechtfertigen wegen der bereits angeführten fehlenden Zweckbindung für Vorsorgezwecke keine allgemeine Begünstigung der Vermögensbildung 27.

Eine besondere Härte und unter steuersystematischen Aspekten nicht gerechtfertigt ist allerdings die Besteuerung von Vermögensrückflüssen, die der Sicherung des Existenzminimums dienen. Eine Erstattung der auf diesen Beträgen lastenden Einkommensteuer ist geboten. Da aber die Berücksichtigung der persönlichen marginalen Steuerbelastung in der Ansparphase zu aufwendig wäre und zusätzlich Annahmen hinsichtlich der zeitlichen Herkunft in Anspruch genommener Vermögensbeträge erforderte, ist eine Pauschalierung unvermeidbar, die etwa in Höhe des Steuersatzes der Eingangsproportionalzone erfolgen könnte28. Eine Berücksichtigung in der Ansparphase ist praktisch unmöglich, da wegen unsicherer individueller Lebenserwartungen weder die benötigte Ansparsumme zu ermitteln ist noch sicher ist, ob nicht andere Einkünfte im Alter vorliegen, die für sich genommen bereits die Inanspruchnahme des Existenzminimum-Freibetrags ermöglichen.

Als Sonderfall der Vermögensbildung kann der Erwerb von Wohneigentum verstanden werden, da hierdurch bereits per se ein Teil der Lebenshaltungskosten im Alter gedeckt wird - jedenfalls bei Nutzung

26 Insbesondere nicht wegen der Ersparnis für eigene Konsumzwecke, sondern für Zwecke des Vermögenstransfers an Dritte. Daher bewegt sich der Vorschlag von Mitschke (1985, 53f., Rz.62), der eine steuerliche Erfassung der bis dahin steuerfreien Sparbeträge im Todesfall vorsieht, im Bereich zwischen Einkommensteuer und persönlicher allgemeiner Verbrauchsteuer.

27 Zur Förderung der sich im Vermögen ausdrückenden privaten Vorsorge, die aber keineswegs zwingend geboten ist, bietet sich neben Freibeträgen innerhalb der Vermögensteuer in Höhe einer als angemessen betrachteten Rücklage für Notfälle allenfalls eine Sparprämie in Form des Abzugs von der Einkommensteuerschuld an. Da die Förderung der allgemeinen Vermögensbildung jedoch geringer als die Förderung der zweckgebundenen Altersvorsorge sein sollte, würde sie mit einiger Berechtigung eine Reihe von Folgeförderungen nach sich ziehen.

28 Überlegungen zur Höhe des zu pauschalierenden Erstattungssatzes wurden bereits angestellt. Siehe Kapitel V.1.2.1. 
durch den Steuerpflichtigen selbst. Allerdings handelt es sich auch hier um frei veräußerbares Vermögen, dessen Zweckbindung für die Altersvorsorge nicht sichergestellt ist. Auch steht die deutsche Tradition dieser Art des "Vorsorgesparens" stark im Zeichen der Förderung des Wohnungsbaus, was nicht gerade für eine Sonderstellung im Rahmen der Altersvorsorge spricht ${ }^{29}$. Besondere Probleme einer Absicherung der Steuerfreiheit des Existenzminimums im Alter ergeben sich hier nicht, da der Nutzungswert einer eigengenutzten Wohnung - sofern er überhaupt steuerlich erfaßt wird - wie andere Einkünfte in die Steuerbemessungsgrundlage eingeht; Veräußerungserlöse bei Wohneigentum sind wie die Erlöse anderer Vermögensveräußerungen zu behandeln.

Die derzeitigen Regelungen im deutschen Einkommensteuerrecht scheinen auf den ersten Blick den hier abgeleiteten Forderungen zu entsprechen: Vermögensbildung ist aus versteuertem Einkommen zu leisten, Einkünfte aus Kapitalvermögen sind einkommensteuerpflichtig. Allerdings sind Beiträge zu Bausparkassen im Rahmen der Vorsorgeaufwendungen und der dort geltenden Höchstbeträge abzugsfähig ( $§ 10(1)$ Nr.3 EStG). Die quantitativ weitaus bedeutenderen Steuerbefreiungen bestehen für Wertzuwächse des Vermögensbestands: im privaten Bereich sind sowohl unrealisierte als auch realisierte Wertzuwächse (Veräußerungsgewinne) grundsätzlich steuerfrei (Tipke/Lang, 1991, 367-370), hinzu kommt eine politisch beabsichtigte (vgl. § 30a AO) Untererfassung von Kapitalerträgen. Im betrieblichen Bereich finden sich nennenswerte Freibeträge und Sondervorschriften für die Versteuerung von Veräußerungsgewinnen, die zum Teil (§ 16(4) EStG) explizit mit dem Argument der Altersvorsorge begründet werden.

29 So zunächst auch Heuer, der gleichwohl in Bausparbeiträgen "gebotene oder zumindest angebrachte Vorsorgemaßnahmen" sieht, die die Leistungsfähigkeit der Steuerpflichtigen minderten (Heuer, 1987, Rz. 150f.). Kritisch gegenüber einer Sonderbehandlung Wissenschaftlicher Beirat beim BMF $(1967,344)$ sowie Steuerreformkommission (1971, 265f., Rz. 26f.). 


\subsubsection{Kapitalversicherungen auf den Erlebensfall}

Bei Kapitalversicherungen auf den Erlebensfall wird eine Versicherungsleistung dann fällig, wenn der Versicherte den vereinbarten Zeitpunkt erlebt. In der Regel wird auch für den Todesfall eine Leistung in Form einer Prämienrückgewähr vereinbart. Im folgenden wird allerdings vor allem die reine Erlebensfallversicherung in Form einer Versicherung auf die eigene Person betrachtet, bei der Versicherungsnehmer (Beitragszahler), Versicherter und Bezugsberechtigter identisch sind 30 .

Bei dieser Art von Versicherungen handelt es sich vor allem um einen Sparvorgang. Die Höhe der Ersparnis entspricht der Veränderung des Rückkaufswerts der Versicherung, da dieser bei Orientierung an der Marktfähigkeit den aktuellen Vermögenswert repräsentiert ${ }^{31}$. Im Rahmen einer umfassenden Einkommensbesteuerung, die auch Wertzuwächse im Privatvermögen erfaßt, sind hier der Veränderung des Rückkaufswerts die aufgewendeten Beiträge gegenüberzustellen 32 . Ebenfalls sind bei Vertragsablauf vom Rückkaufswert abweichende Leistungen einkommensteuerlich zu berücksichtigen 33 .

Als Altersvorsorge sind Kapitalversicherungen auf den Erlebensfall nur sehr bedingt geeignet. Zunächst müßte der Erlebens-(Versicherungs-) fall auch tatsächlich auf einen Zeitpunkt in der "Altersphase", also (mindestens) auf das 60. Lebensjahr oder später, datiert sein, außerdem der Vertrag auf die eigene Person abgeschlossen sowie eine Leistung

30 Vgl. zu den übrigen Fällen das Kapitel "Versicherungen zugunsten Dritter" (V.1.3.5), zur Terminologie Hagelschuer (1983, 61-65).

31 Vgl. hierzu auch § 12(4) BewG.

32 Allenfalls eine Korrektur von Versicherungsbeiträgen um den im Vergleich zur Ersparnis erhöhten Verwaltungsaufwand des Versicherungsunternehmens erscheint denkbar, jedoch ist eine Präzisierung der Spar-Alternative mit den dort anfallenden Werbungskosten nur schwer möglich.

33 Im hier betrachteten Fall der Kapitalversicherung auf den Erlebensfall dürften Verluste nur bei vorzeitigem Tod des Versicherten eintreten, da Leistungen im Todesfall entweder ausgeschlossen sind oder unter dem Rückkaufswert liegen. Hier wäre ein Anrecht der Erben auf Berücksichtigung der Wertminderung in der bzw. den letzten Einkommensteuererklärungen des Verstorbenen sicherzustellen. 
(z.B. Prämienrückgewähr) im Todesfall und eine Vorausverfügung über die zu erwartende Kapitalsumme ausgeschlossen sein. Aber auch dann handelt es sich noch um eine sehr unzweckmäßige Form der Alterssicherung, da der Tod der auf diese Art durch eine Kapitalsumme abgesicherten Person bereits kurz nach Auszahlung der Versicherungssumme, aber auch erst Jahrzehnte später eintreten kann. Für steuerliche Zwecke bieten sich weder für eine Steuerbefreiung des Existenzminimums im Alter noch für die Berücksichtigung von Aspekten einer Lebenseinkommensbesteuerung günstige Anknüpfungspunkte. Obwohl es sich hier genauso wie bei Leibrentenversicherungen um Versicherungen auf den Erlebensfall handelt, bestehen weitaus größere Gemeinsamkeiten mit der allgemeinen Vermögensbildung. Eine besondere Förderung von Kapitalversicherungen auf den Erlebensfall erscheint daher kaum angemessen. Da der Rückkaufswert allerdings aufgrund einer unvollständigen Erfassung von Überschußanteilen und eines Stornoabzugs vom Deckungskapital regelmäßig unter dem Zeitwert des Versicherungsanspruchs bei Erfüllung der Vertragslaufzeit liegt, ergibt sich eine implizite Förderung in Höhe des Zinsvorteils durch Aufschub der Besteuerung der Differenz zur Wertentwicklung des Versicherungsanspruchs ${ }^{34}$.

Leibrentenversicherungen mit Kapitalwahlrecht sind, jedenfalls bei Ausübung dieses Wahlrechts, den Kapitalversicherungen auf den Erlebensfall steuerlich gleichzustellen. $\mathrm{Da}$ in diesem Bereich Rückkaufswerte nicht üblich sind (Hagelschuer, 1983, 88), ist deren einkommensteuerliche Behandlung nicht klar vorgezeichnet. Am ehesten bietet sich ein Verfahren an, das die Versicherungsbeiträge zunächst als Ersparnis behandelt, bei Inanspruchnahme der Kapitalsumme oder vorzeitigem Tod die Wertveränderung erfaßt und im Falle der definitiven Festlegung auf

34 Dieser Zinsvorteil ließe sich nur vermeiden, wenn für einkommensteuerliche Zwecke neben dem Rückkaufswert auch die tatsächliche Wertentwicklung des Versicherungsanspruchs für den Versicherungsnehmer ausgewiesen würde. Falls Liquiditätsaspekten ein hoher Stellenwert eingeräumt würde, könnte die auf die Differenz entfallende Steuerschuld ggf. gestundet werden. Andererseits wirkt die stärkere Steuerprogression infolge hoher Überschußanteile bei Vertragsablauf tendenziell diesem Effekt entgegen. 
eine Leibrentenversicherung das bis dahin aufgelaufene Sparkapital als Einmalbeitrag für diese Versicherung verbucht. Im Kapitel, das sich den Leibrentenversicherungen widmet, wird darauf zurückzukommen sein.

Bei den zur Zeit in Deutschland vorwiegend anzutreffenden Versicherungen auf den Erlebens- und Todesfall ${ }^{35}$ ist ggf. eine fiktive rechnerische Aufteilung in zwei Versicherungsverträge - eine Kapitalversicherung auf den Todesfall und eine Kapitalversicherung auf den Erlebensfall - vorzunehmen, um dann die hier abgeleiteten Regeln der einkommensteuerlichen Behandlung auf diese Teilverträge anzuwenden. Die notwendigen Kalkulationsgrundlagen liegen ohnehin vor, da die Versicherungsunternehmen sie für ihre Beitragskalkulation verwenden. Die vorgelegten Daten sind vom Bundesaufsichtsamt für das Versicherungswesen zu überprüfen.

Die derzeitige einkommensteuerliche Regelung sieht vor, daß Beiträge zu Kapitalversicherungen (ebenso wie zu Rentenversicherungen mit Kapitalwahlrecht) dann im Rahmen der für Vorsorgeaufwendungen insgesamt geltenden Höchstbeträge als Sonderausgaben abgezogen werden können, wenn die Vertragslaufzeit mindestens 12 Jahre beträgt und eine laufende Beitragsleistung erfolgt ( $\$ 10(1)$ Nr.2b EStG). Zinsen auf in den Beiträgen enthaltene Sparanteile sind zwar nach § 20(1) Nr.6 EStG grundsätzlich steuerpflichtig, dies gilt jedoch i.d.R. nicht für Versicherungen, die die Bedingungen nach $\S 10$ (1) Nr.2b EStG erfüllen, so daß in den allermeisten Fällen die gesamte Versicherungsleistung steuerfrei bleibt.

\subsubsection{Kapitalversicherungen auf den Todesfall}

Bei Kapitalversicherungen auf den Todesfall wird die vereinbarte Versicherungssumme im Fall des Todes des Versicherten fällig. Dem Versicherten steht die Wahl eines Bezugsberechtigten für die Versicherungssumme frei. Tritt der Tod des Versicherten nicht innerhalb der

35 Die Beliebtheit dieser Versicherungsform ist neben dem gebotenen Leistungsmix maßgeblich auf deren derzeitige einkommensteuerliche Behandlung zurückzuführen. 
Vertragslaufzeit ein, ist keine Leistung vorgesehen. Sofern die Vertragslaufzeit unbegrenzt ist, was allerdings die Ausnahme darstellt, enthält auch die Todesfallversicherung "Sparelemente", da der Versicherungsfall dann früher oder später garantiert eintritt. Die Verzinsung der Beiträge wird mit zunehmendem Alter des Versicherten allerdings immer geringer - unter Umständen sogar negativ - ausfallen. Ist dagegen die Vertragslaufzeit begrenzt, dann führt nur ein geringer Teil der Versicherungsverträge zu einer Auszahlung.

Bei der Todesfallversicherung handelt es sich nicht um Vorsorge für den Versicherten selbst - bei Eintritt des Versicherungsfalls ist für diesen eine Vorsorge nicht mehr nötig -, sondern für Dritte. Grundsätzlich ist daher eine steuerliche Berücksichtigung aus Motiven der Alterssicherung abzulehnen; Beiträge zu Todesfallversicherungen sind aus versteuertem Einkommen zu leisten.

Eine zeitgleiche einkommensteuerliche Erfassung beim Bezugsberechtigten kann allerdings nicht realisiert werden, da die alleinigen Vertragsrechte i.d.R. beim Versicherungsnehmer verbleiben, was diesem sowohl eine Kündigung bzw. den Rückkauf des Vertrags als auch eine Änderung der Person des Bezugsberechtigten ermöglicht. Bei den gegenwärtig vorherrschenden Versicherungen auf den Erlebens- und Todesfall ist eine Aufteilung des Beitrags auf die beiden Versicherungsformen notwendig, weil sonst die anteiligen Beiträge zur Todesfallversicherung implizit als Wertminderung steuerlich geltend gemacht werden könnten 36 .

Ob der Tod des Versicherten einen materiellen Bedarf für dritte Personen auslöst, wenn ja, für welchen Zeitraum und in welcher Höhe, und wie eine adäquate einkommensteuerliche Behandlung solcher Versicherungen aussehen könnte, wird später im Kontext der einkommensteuerlichen Behandlung von Transfers zwischen Privaten, insbesondere innerfamiliärer Transfers, noch näher zu klären sein. Einer Kapital-

36 Vgl. zu diesem Gesamtkontext auch die Ausführungen bei Vickrey (1947, 64-66), die sich im Ergebnis dadurch unterscheiden, daß bei Vickrey die Transferanteile steuerfrei bleiben. 
summe in beliebiger Höhe fehlt allerdings schon von der Konzeption her ein Bedarfsbezug, sie hat eher den Charakter eines Lottogewinns 37.

Abgesehen von diesen Überlegungen zur steuerlichen Behandlung der Beiträge bleibt festzuhalten, daß die Versicherungsleistungen jedenfalls die Leistungsfähigkeit der Begünstigten erhöhen und daher voll zu versteuern sind. Beispielsweise durch den Tod des "Ernährers" mögen zwar individuelle Nachteile eintreten, Gegenstand der Einkommensteuer ist jedoch die aktuelle steuerliche Leistungsfähigkeit, nicht deren Veränderung zum Vorjahr. Andere Argumentationen lassen sich lediglich auf einen unter allgemeinen Leistungsfähigkeitsgesichtspunkten kaum zu rechtfertigenden Mitleidseffekt zurückführen.

Die derzeitige einkommensteuerliche Behandlung ist bei Todesfallversicherungen weniger restriktiv als bei Erlebensfallversicherungen, wohl weil der Gesetzgeber einen "Mißbrauch" als Vermögensbildungsform für ausgeschlossen hält. So ist weder eine laufende Beitragszahlung noch eine bestimmte Mindestlaufzeit Bedingung für eine Qualifikation der Beiträge als Sonderausgaben nach $\S 10(1)$ Nr.2b EStG. Die Versicherungsleistungen sind beim Bezugsberechtigten nicht einkommensteuerpflichtig, unterliegen allerdings der Erbschaftsteuer (Kalwar, 1988, 833).

\subsubsection{Rentenversicherungen}

\subsubsection{Einkommensteuersystematische Einordnung}

\section{(1) Charakterisierung}

Bei einer Rentenversicherung verpflichtet sich der Versicherer zu laufend wiederkehrenden Zahlungen ab einem bestimmten Zeitpunkt, dem Rentenbeginn, bis zum Lebensende des Versicherten (Leibrente) oder auch für einen festgelegten Zeitraum (Zeitrente). Beitragszahlungen zur Finanzierung sind grundsätzlich sowohl über einen längeren Zeitraum hinweg als auch als Einmalbeitrag möglich.

37 Das erklärt wohl auch die Beliebtheit von Todesfallversicherungen als offenbar jedermann einleuchtendes Mord- und Bereicherungsmotiv in Kriminalromanen. 
Die Leibrente ist das adäquate Instrument der Altersvorsorge, da sie einen gleichmäßigen Mittelzufluß für die gesamte Lebensphase der altersbedingten Nichterwerbstätigkeit sicherstellt. Es können allerdings inflationsbedingte Probleme auftreten, sofern keine entsprechenden Anpassungen (Dynamisierung) vorgesehen sind. Zeitrenten eignen sich hingegen für die Absicherung von Bedarfssituationen, die in voraussehbarem Umfang zeitlich begrenzt sind, wie das beispielsweise bei Ausbildungszeiten oder Zeiten der Kindererziehung der Fall ist. Wegen der nicht allgemein vorhersehbaren individuellen Lebensdauer sind sie für Zwecke der Alterssicherung vergleichsweise ungeeignet und werden daher hier nicht weiter betrachtet. Leibrenten ermöglichen in nahezu optimaler Weise eine Verwirklichung der am Anfang dieses Kapitels behandelten Forderungen an eine effektive Altersvorsorge, nämlich den gleichmäßigen Zufluß von Alterseinkommen und damit eine Sicherung des Existenzminimums über die gesamte Altersphase (sofern nur die Beitragszahlungen ausreichend hoch waren) bei gleichzeitiger Kalkulierbarkeit des notwendigen Aufwands, was eine einkommensteuerliche Freistellung des Existenzminimums im Alter problemlos ermöglicht.

Aus diesen Gründen sollen im weiteren als Modellfall Leibrentenversicherungen ohne Kapitalwahlrecht betrachtet werden, da bei Vorliegen eines Kapitalwahlrechts noch keine definitive Festlegung auf eine Leibrentenversicherung erfolgt ist. Zeitrenten haben einen festen Wert und sind somit ohnehin als allgemeine Vermögensbildung einzustufen.

Für die Frage der einkommensteuerlichen Behandlung wird der traditionellen Sicht (Beiträge als Ersparnis) die in dieser Arbeit vertretene Sicht (Beiträge als Werbungskosten) gegenübergestellt. Zunächst scheint es sich hier um eine rein terminologische Diskussion zu handeln; diese hat jedoch Auswirkungen auf die Einordnung des Tatbestands in das Einkommensteuerrecht im allgemeinen und auf die institutionelle Abgrenzung derjenigen Altersvorsorgeaufwendungen, die verfahrenstechnisch gleich behandelt werden. 
(2) Traditionelle Sicht: Leibrentenversicherung als Vermögensbildung

Traditionell werden Leibrentenversicherungsbeiträge als eine besondere Form der Ersparnis eingestuft 38 . Bei bekannter Lebenserwartung könnte in der Tat eine Leibrentenversicherung ebenso gut durch private Vermögensbildung und -auflösung realisiert werden. Bei ex ante unbekannter Lebenserwartung läßt sich diese Charakterisierung des Sachverhalts zwar auf die mit Überlebenswahrscheinlichkeiten kalkulierende Institution Versicherung übertragen, jedoch nicht auf die Ebene der einzelnen Beitragszahler. Für diese stellt sich die Situation im Vergleich zur Vermögensbildung vollkommen verschieden dar: "Angespart" werden lediglich aufgrund der ex ante unbekannten Lebensdauer individuell ungewisse Ansprüche, deren jeweiliger Erwartungswert nicht realisiert werden kann. Die Summe (genauer: der Barwert) der tatsächlichen Rentenzahlungen ist nicht beschränkt, sondern diese werden auf Grundlage der einmal bei Renteneintritt berechneten Höhe über die gesamte individuelle Lebenszeit des Versicherten hin geleistet.

Wird gleichwohl an der traditionellen Sichtweise der Rentenversicherungsbeiträge als Ersparnis festgehalten, wofür auch die Proklamierung eines Eigentumsschutzes für Rentenanwartschaften durch das Bundesverfassungsgericht $(1980,257,289 \mathrm{f}$; 1985b, 272) zu sprechen scheint, bleiben jedenfalls bereits im Modell Probleme der einkommensteuerlichen Behandlung, die sich aus der ex ante ungewissen individuellen Lebenserwartung ergeben. Dabei geht es um die Sicherstellung einer steuerfreien Auflösung von Vermögensbeständen, die steuerliche Erfassung von Vermögenserträgen in Form von Zinsen und Umverteilungsgewinnen sowie die Realisierung einer gleichmäßigen Steuerbelastung in der Altersphase. Verschiedene Lösungsvorschläge werden im folgenden vorgestellt.

\section{(a) Normalbesteuerung}

Das Verfahren, das der üblichen Besteuerung von ertragbringenden Vermögensanlagen am nächsten kommt, ist folgendes: Ebenso wie jede

38 Vgl. hierzu Vickrey (1947, 75f.), Andel (1970), Haller (1973, 522-527), Albers $(1980,200)$. Auch der deutsche Gesetzgeber läßt sich hier anführen, der in § $22 \mathrm{Nr}$.1 EStG zwischen Kapital- und Zinsanteil trennt. 
andere Ersparnis sind Rentenversicherungsbeiträge als Einkommensverwendung $\mathrm{zu}$ betrachten und daher aus versteuertem Einkommen $\mathrm{zu}$ bestreiten. Wertänderungen der Rentenanwartschaft durch Zinsen sowie durch versicherungsimmanente Umverteilung (Tod anderer Versicherter) werden dem Versicherungsnehmer laufend zugerechnet ${ }^{39}$. Als steuerpflichtige Einkünfte sind sie grundsätzlich einkommensteuerpflichtig. Die späteren Rentenzahlungen bleiben als Vermögensauflösungen steuerfrei. Problematisch ist der Fall von Wertminderungen durch Tod des Versicherungsnehmers: Der Verlust durch Erlöschen des Rentenanspruchs kann einkommensteuerlich nicht mehr geltend gemacht werden, da nach dem Tod die persönliche Einkommensteuer nicht mehr erhoben wird. Eine Möglichkeit der einkommensteuerlichen Berücksichtigung solcher Verluste besteht allenfalls in der Revision der Steuerbescheide der letzten Jahre, was sich aber nur noch zugunsten der Erben auswirken kann 40 . Ebenfalls problematisch ist, daß die Regelmäßigkeit des Mittelzuflusses, wie sie eine Leibrentenversicherung grundsätzlich über die Aktivenphase hinaus ermöglicht, keine Entsprechung in der Verteilung und Bemessung der Einkommensteuerschuld findet. Die Einkommensteuerbelastung liegt von der Besteuerung von Zinsen und Umverteilungsgewinnen abgesehen allein in der Phase der Beitragszahlung, so daß eine gleichmäßige Besteuerung des Lebenseinkommens verfehlt wird. In der Aktivenphase ist die Steuerprogression überhöht, während gleichzeitig in der Altersphase nicht sichergestellt ist, daß wenigstens der steuerliche Existenzminimum-Freibetrag ausgeschöpft werden kann 41 .

Ein Anreiz zur grundsätzlich erwünschten Altersvorsorge in Form einer Rentenversicherung könnte hier in steuersystematisch akzeptabler Form am ehesten durch eine Förderprämie sichergestellt werden. Ein Freibetrag z.B. für Beiträge, wie sie voraussichtlich für eine spätere

$39 \mathrm{Zu}$ entsprechenden Kalkulationen des Deckungskapitals vgl. Hagelschuer (1983, 140-147).

40 Vgl. zur subjektiven Steuerpflicht Herrmann/Heuer/Raupach (1976, Rz.16). Skeptisch gegenüber einer Berücksichtigung von Verlusten im Todesfall Pollak $(1979,266$, Fn.11) sowie Richter $(1987,669)$.

$41 \mathrm{Zu}$ möglichen Lösungen dieses Problems im Einzelfall vgl. Kapitel V.1.2.1. 
Rente in Höhe des Existenzminimums notwendig sind, wäre nur dann angebracht, wenn die Leistungsfähigkeit der Steuerpflichtigen in Höhe des Beitrags als gemindert betrachtet werden müßte, was aber der Ausgangsüberlegung dieses Modells, der Charakterisierung der Rentenversicherung als Vermögensbildung, widerspricht. Auch wäre dann eine Inanspruchnahme des Existenzminimumfreibetrages der Altersphase je nach Vorliegen anderer bzw. darüber hinausgehender Einkünfte doppelt möglich. Soll ein Vermerk in den Steuerakten dies verhindern, so bringt das erhebliche Schwierigkeiten mit sich 42 .

\section{(b) Summarische Erfassung von Zinserträgen und Umvertei- lungsgewinnen bei Auszahlung}

Auch hier sind prinzipiell Beiträge zu Rentenversicherungen aus versteuertem Einkommen zu leisten, allerdings werden Zinserträge und Umverteilungsgewinne nicht bereits im Zeitpunkt ihrer Entstehung dem Versicherungsnehmer zugerechnet. Eine Besteuerung dieser Komponenten erfolgt erst zum Zeitpunkt des Zuflusses beim Versicherungsnehmer, was zwar wegen des damit verbundenen Zinseffektes steuersystematisch nicht korrekt, aber erheblich einfacher durchzuführen ist ${ }^{43}$.

Für die Phase der Rentenzahlung muß dann allerdings für einkommensteuerliche Zwecke eine Reihenfolge des Zuflusses der Komponenten Beitragsvermögen, Zinserträge und Umverteilungsgewinne konstruiert werden, da die erste steuerfrei ist, die beiden anderen jedoch steuerpflichtig sind (Andel, 1970, 334f.). Im Extremfall kann sich so ein Belastungssprung von voller Steuerfreiheit des Rentenzahlbetrages (wenn zunächst die Rückzahlung des Beitragsvermögens unterstellt wird) zu voller Steuerpflicht (ab dem Zeitpunkt, ab dem der gesamte Rentenzahl-

42 Dies hängt vor allem mit der Langfristigkeit der Wirkungen zusammen und mit der Notwendigkeit, in "Härtefällen" von dieser Linie abzugehen. Hinzu kommen verfahrenstechnische Probleme wie die Bestimmung der notwendigen Altersvorsorge in Relation zum zukünftigen Existenzminimum und Probleme der Steuertransparenz, wenn gleich hohe Periodeneinkommen unterschiedlich behandelt werden.

43 Die Verwendung des Realisationsprinzips zeigt hier Parallelen zur derzeitigen steuerlichen Erfassung von Veräußerungsgewinnen im betrieblichen Bereich. 
betrag sich nur noch aus den Komponenten Zinserträge und Umverteilungsgewinne zusammensetzt) oder umgekehrt ergeben. Auch die Orientierung an der durchschnittlichen Lebenserwartung für die Festlegung der Rückzahlungsraten des aus Beiträgen angesammelten Kapitals führt nicht dazu, daß ein Steuerbelastungssprung vermieden wird, mildert ihn jedoch $^{44}$.

Gemeinsam ist diesen Varianten, daß die Berücksichtigung von Umverteilungsverlusten durch Tod des Versicherten auf die bereits genannten Schwierigkeiten stößt. Zu Lasten einer konsequent systematischen Ausgestaltung in bezug auf die einkommensteuerliche Erfassung der Ersparnis wird allerdings eine tendenziell gleichmäßigere Besteuerung des Lebenseinkommens als bei der "Normalbesteuerung" nach (a) erreicht und auch die Gefahr eines faktischen Versagens des steuerlichen Grundfreibetrags in Höhe des Existenzminimums reduziert.

\section{(c) Ertragsanteilsbesteuerung}

Der Ertragsanteilsbesteuerung liegt der Idee nach die Zerlegung einer auf einem Rentenstammrecht beruhenden Zeitrente in Zinsertrag und Vermögensrückfluß zugrunde. Ziel dieser gedanklichen Konstruktion ist die steuerliche Erfassung des Ertrags des Rentenrechts in Analogie zu anderen Vermögenserträgen. Der Erwerb des Rentenstammrechts ist dabei nicht mehr Gegenstand der Betrachtung. Da die Ertragsanteilsbesteuerung auf durch Hingabe eines einmaligen Kapitalbetrages erworbene Zeitrenten zugeschnitten ist, kann ein Erwerb des Rentenstammrechts aus vorhandenem Vermögen unterstellt werden, dessen einkommensteuerliche Behandlung abgeschlossen ist. Für Leibrentenversicherungen gegen laufende Beitragszahlung trifft dies aber nur dann zu, wenn die Beiträge aus versteuertem Einkommen geleistet und insbesondere auch bis $\mathrm{zu}$ diesem Zeitpunkt angefallene Zinserträge und die versicherungsimmanente Umverteilung durch den Tod von Versicherten einkommensteuerlich berücksichtigt wurden. Auch nach dem Beginn des Rentenbezugszeit-

44 Die derzeitige einkommensteuerliche Regelung vermeidet einen solchen Belastungssprung nur dadurch, daß sie den einmal festgelegten Ertragsanteil auch dann noch beibehält, wenn das Beitragskapital der Konstruktion nach bereits voll zurückgezahlt ist. 
raums bleiben Umverteilungsvorgänge in Leibrentenversicherungen grundsätzlich bei allen Ertragsanteilsbesteuerungsverfahren einkommensteuerlich unbeachtet, da sie bezüglich der die Laufzeit bestimmenden Lebenserwartung mit einheitlichen, lediglich die Ex-ante-Erwartungen widerspiegelnden, Werten arbeiten (Richter, 1987, 668f.) ${ }^{45}$. Hier sowie in der Nichtbeachtung von dynamisierten Rentenzahlungen, die in der privaten Leibrentenversicherung eine gewisse Analogie in der Überschußbeteiligung finden, liegen die größten Fehler der Ertragsanteilsbesteuerung, die grundsätzlich bei Anwendung auf private und gesetzliche Leibrenten gleichermaßen auftreten ${ }^{46}$. Rein praktische Probleme einer sachgerechten Umsetzung liegen in der richtigen Abschätzung von Kapitalmarktzins und Lebenserwartung.

Die in Deutschland für Leibrenten übliche Ertragsanteilsbesteuerung nach § $22 \mathrm{Nr} .1$ EStG ergibt sich aus der gleichmäßigen Verteilung der bei durchschnittlicher Lebenserwartung anfallenden Zinserträge auf die erwartete Rentenlaufzeit. Dieser Ertragsanteil wird dann unabhängig von der tatsächlichen Rentenbezugsdauer beibehalten. Die Beiträge sind im Rahmen der für Vorsorgeaufwendungen geltenden Höchstbeträge als Sonderausgaben nach § 10(1) Nr.2a, 2b EStG abzugsfähig, sofern es sich um Beiträge zu einer gesetzlichen Rentenversicherung oder einer privaten Rentenversicherung ohne Kapitalwahlrecht handelt oder die Mindestvertragsdauer 12 Jahre beträgt und eine laufende Beitragszahlung vorgesehen ist.

45 Eine von Richter $(1987,680 f ., 684)$ erwähnte, jedoch letztlich verworfene Ertragsanteilsbesteuerung ex post entspricht allerdings ab dem Zeitpunkt des Rentenbezugs dem unter (a) beschriebenen Verfahren der Normalbesteuerung.

46 Andel $(1970,341)$ hält dagegen die Anwendung der Ertragsanteilsbesteuerung (nach $\S 22 \mathrm{Nr} .1 \mathrm{EStG}$ ) auf private Leibrenten für vertretbar, insb. wohl auch deshalb, weil er bei privaten Versicherungen von kürzeren Zeiträumen zwischen (erster) Beitragszahlung und Rentenbeginn ausgeht. Dies ist aber nicht zwangsläufig so; außerdem vernachlässigt er die von einer Ex-post-Besteuerung $\mathrm{zu}$ erfassenden Umverteilungsgewinne und -verluste, die eine quantitativ etwa ebenso bedeutende Größenordnung annehmen wie die Zinserträge bzw. die Dynamisierung. 
Ein davon abweichendes Verfahren, die Ertragsanteilsbesteuerung nach Maßgabe des ökonomischen Ertrags im Sinne von Hicks, wird von Richter (1987) vorgeschlagen: Bei diesem Verfahren sind die Ertragsanteile im Zeitablauf nicht konstant, sondern nehmen wegen zunehmender Bedeutung von "Abschreibungen" auf das Rentenstammrecht mit steigendem Alter des Steuerpflichtigen ab. In allen übrigen Eigenschaften stimmen die beiden Ertragsanteilsbesteuerungsverfahren überein.

Schließlich beruht ein von Schmähl (1981, 281-298) insbesondere für die Anwendung auf Sozialversicherungsrenten entwickeltes Modell einer "modifizierten Ertragsanteilsbesteuerung" auf ähnlichen Überlegungen. Es unterscheidet sich von den beiden skizzierten Verfahren vor allem dadurch, daß von der verfahrenstechnischen Vorgehensweise her nicht ein Ertrags-, sondern ein Tilgungsanteil der Rente bestimmt wird. Dies beinhaltet eine Steuerpflicht der vor Beginn der Rentenlaufzeit angefallenen Zinserträge und der bis zu diesem Zeitpunkt eingetretenen versicherungsimmanenten Umverteilungen 47 . Diese Tilgungsanteile und somit auch die dazu komplementären Ertragsanteile werden jeweils bei Renteneintritt bestimmt und bleiben danach konstant. Dadurch werden später auftretende versicherungsimmanente Umverteilungsprozesse nicht mehr steuerlich erfaßt und im Zeitablauf absolut steigende Tilgungsbeträge zugelassen, was sachlich nicht gerechtfertigt ist 48 .

\section{(d) Steuerstundung}

Aus dem Umstand des Zwangs zur Beitragszahlung an Alterssicherungssysteme, insbesondere an die gesetzliche Rentenversicherung, wird in einem Teil der Literatur bereits eine Minderung der Leistungsfähigkeit

47 Ebenso wird die sich in der Rentenberechnung der gesetzlichen Rentenversicherung ausdrückende interpersonelle Umverteilung (zur Begriffsklärung vgl. Andel, 1990, 429f.) steuerpflichtig, die allerdings in der privaten Rentenversicherung keine Rolle spielt.

48 Hinzu kommt - wiederum nur für die gesetzliche Rentenversicherung - die Nichterfassung intergenerationaler Umverteilung, die sich in veränderten Beitragssätzen ausdrückt, da die Bestimmung von fiktiven Beitragszahlungen mit Hilfe des bei Renteneintritt gerade gültigen Beitragssatzes erfolgt. 
der Steuerpflichtigen im Umfang solcher Beiträge geschlossen. Liquiditätsgesichtspunkten wird hier offensichtlich ein solch hoher Stellenwert eingeräumt. Bei Klassifikation der Beiträge als Ersparnis bietet sich dann aber eine Steuerstundung als unter systematischen Aspekten überlegenes Verfahren an.

Im Fall der Steuerstundung sind Beiträge zu Leibrentenversicherungen prinzipiell steuerpflichtig, die darauf entfallende Steuerschuld wird aber bis zum Zufluß von Rentenzahlungen gestundet ${ }^{49}$. Dadurch wird konsequent sowohl die Behandlung von Rentenversicherungsbeiträgen als Ersparnis als auch eine Berücksichtigung der durch die Beitragszahlungen verminderten Verfügungsmöglichkeiten über Einkommen während der Aktivenphase erreicht. Zudem kann Umverteilungsverlusten in pauschaler Form dadurch steuerlich Rechnung getragen werden, daß im Falle des Todes von Steuerpflichtigen noch verbleibende Steuerschulden aus der Stundung erlassen und nicht etwa zu Lasten der Erben eingefordert werden. Rentabilitätsvorteile der "Anlageform Leibrentenversicherung" gegenüber anderen Sparformen werden - die Wahl eines marktüblichen Zinssatzes zur Verzinsung der gestundeten Steuerschuld vorausgesetzt - vermieden. Allerdings wird weder eine Annäherung an das Konzept einer Lebenseinkommensbesteuerung erreicht, noch eine Ausschöpfung des steuerlichen Existenzminimumfreibetrages im Alter gewährleistet, da die Steuerschuld bereits im Zeitpunkt der Beitragszahlung bzw. des Anfalls von Erträgen festgesetzt wird.

Im Detail muß so vorgegangen werden, daß auf Beiträge zu bzw. Zinsen und Umverteilungsgewinne von Leibrentenversicherungen der

49 Der Tatbestand der Steuerstundung ist präziser als der einer "Nachversteuerung", zumal wenn der Zweck einer Steuerfreiheit von Sonderausgaben nicht abschließend geklärt ist. Vgl. zur juristischen Diskussion einer "Nachversteuerung" mit dem Ziel einer Durchsetzung des Korrespondenzprinzips Birk (1986b, 138f.) und Söhn (1986, 331-333). 
individuelle Steuersatz des jeweiligen Jahres angewendet wird50. Dazu sind als Grundlage jährliche Bescheinigungen der Versicherungsunternehmen über die Wertentwicklung des Vertrags, das Deckungskapital und die angefallenen Überschußbeteiligungen 51 , nötig. In der Leistungsphase der Rentenversicherung müßte dann die aufgelaufene - und sich durch weiter anfallende Zinsen und Umverteilungsgewinne noch vergrößernde Steuerschuld getilgt werden. Die zeitliche Verteilung der Tilgung der Steuerschuld ergibt sich nicht zwangsläufig; am plausibelsten erschiene mir eine anteilige Tilgung gemäß dem Anteil der Jahresrente am jeweils aufgelaufenen Deckungskapital ("Vermögenswert") der Versicherung. Beim Tod des Versicherten würde der Verlust an Deckungskapital seine Entsprechung im Erlaß des anteilig darauf entfallenden Steuerbetrags finden.

\section{(e) Anwendung des "Korrespondenzprinzips der nachgelagerten Art"}

Beim "Korrespondenzprinzip der nachgelagerten Art" werden die Beiträge zu Leibrentenversicherungen steuerfrei gelassen, erst die ausgezahlten Renten werden voll in die Steuerbemessungsgrundlage eingerechnet und entsprechend besteuert ${ }^{52}$. Eine solche Vorgehensweise ermöglicht sowohl eine Umschichtung des steuerlichen Lebenseinkommens als auch die Ausschöpfung des Existenzminimum-Grundfreibetrages im Alter sowie eine Anerkennung verminderter Steuerzahlungsfähigkeit in der Periode der Beitragszahlung, auch führt sie nicht zu einer

50 Ungeklärt bleibt, ob der marginale oder der durchschnittliche Steuersatz sachgerecht ist, da lediglich klar ist, daß der auf die Vorsorgeaufwendungen entfallende Teil der Steuerschuld gestundet werden soll. Im einen Fall ist der zu stundende Betrag als Differenz aus der Steuerschuld unter Einbeziehung der fraglichen Einkommensteile und der Steuerschuld bei verkürzter Bemessungsgrundlage, im anderen als Produkt aus individuellem Durchschnittssteuersatz und den entsprechenden Teilen der Bemessungsgrundlage zu ermitteln.

51 Zur Terminologie vgl. Hagelschuer (1983, 140-175).

52 In der Literatur ist die Meinung fast einhellig zugunsten einer Anwendung des Korrespondenzprinzips der nachgelagerten Art, so beispielsweise Andel (1970, 340), Lamers (1975, 176-178), Littmann (1983, 483-500), Wissenschaftlicher Beirat beim BMF $(1986,520 \mathrm{f} ., 553)$ sowie Groß (1988, 130-133). 
einseitigen steuerlichen Erfassung von Vermögensgewinnen bei Nichtberücksichtigung von Vermögensverlusten.

Allerdings steht eine solche Lösung in deutlichem Widerspruch zu einer systemgerechten (und auch sonst prinzipiell im deutschen Steuerrecht vorgesehenen) Behandlung der Ersparnis. Mit der Prämisse, daß Leibrentenversicherungen eine Form der Ersparnis darstellen, läßt sich eine derartige Regelung nicht vereinbaren. Da die Ersparnis als eine Form der Einkommensverwendung Teil der steuerlichen Bemessungsgrundlage ist, läßt sich eine volle oder auch nur partielle Steuerbefreiung aus steuersystematischer Sicht konsequenterweise nur als Ausdruck einer gewollten Förderung einer bestimmten Einkommensverwendung verstehen 53 . Anderenfalls wäre im Rahmen einer Sonderregelung für Leibrentenversicherungen gleichzeitig die Steuerbefreiung von Beiträgen und die steuerliche Erfassung von Zuflüssen zu regeln. Im derzeitigen Einkommensteuerrecht ist eine solche Sonderregelung nicht erkennbar. Daher ist es verständlich, daß eine Besteuerung des Vermögensrückflusses, wie sie das "Korrespondenzprinzip der nachgelagerten Art" fordert, in einem Teil der Literatur (Schmähl, 1981, 269f., insb. Fn.187; Birk, 1986b, 138f.) abgelehnt wird, zumal im deutschen Einkommensteuerrecht die Motive des Gesetzgebers bei Einführung des Sonderausgabenabzugs schwerlich in der späteren Erfassung des Vermögensrückflusses bestanden haben dürften. Weitere Überlegungen betreffen den Renditevorteil von Leibrentenversicherungen gegenüber anderen Sparformen, der aus Ex-ante-Sicht durch den oben dargestellten Steueraufschub bewirkt wird und damit geeignet ist, die diesbezüglichen Entscheidungen der Steuerzahler zu beeinflussen, womit die Einkommensbesteuerung nicht mehr neutral bezüglich verschiedener Anlageformen ist. Bei einer Rechnung in realen Größen, die eine Realeinkommensbesteuerung voraussetzt ${ }^{54}$, laufender Beitragszahlung in gleichbleibender Höhe über 40 Jahre hinweg, einem Steuersatz auf die von der zeitlichen Verschiebung betroffenen Einkommensteile von 20\% (30\%)

53 Im Gegensatz zur Steuerstundung, wo eine Steuerpflicht besteht und lediglich die Begleichung der Steuerschuld aufgeschoben wird.

54 Vgl. Kapitel II.2.6. 
und einem Realzinssatz von $2 \%$ errechnet sich eine dem Steuervorteil durch Aufschub der Besteuerung äquivalente Prämie auf den Vorsorgebetrag in Höhe von $9,8 \%(13,8 \%)$, bei einem Realzinssatz von $3 \%$ in Höhe von $14,5 \%(22,2 \%)$. Der sich so errechnende Steuervorteil könnte als Förderprämie für eine eigenständige Alterssicherung gerechtfertigt werden 55 und insofern steuerpolitisch willkommen sein. Die Förderung bliebe allerdings nicht auf die Höhe des Existenzminimums beschränkt.

\section{(f) Zusammenfassende Beurteilung}

Zusammenfassend läßt sich sagen, daß in bezug auf die Kriterien periodengerechte Erfassung der Ersparnis sowie von positiven wie negativen Wertveränderungen, Berücksichtigung der in der Periode der Beitragsentrichtung geminderten steuerlichen Zahlungsfähigkeit, Umschichtung des steuerlichen Lebenseinkommens und Gewährleistung der Steuerfreiheit des Existenzminimums im Alter keines der vorgestellten Verfahren eindeutig überlegen ist. Es liegt ein Zielkonflikt zwischen der periodengerechten Erfassung steuerlicher Leistungsfähigkeit in Form von Ersparnis sowie positiven Wertveränderungen und den übrigen Kriterien vor. Dieser Konflikt liegt darin begründet, daß die Anforderungen der ersten Gruppe nur bei sofortiger Erfassung der steuerlichen Leistungsfähigkeit durch eine umfassende Definition der einkommensteuerlichen Bemessungsgrundlage erfüllt werden können, während die Anforderungskriterien der zweiten Gruppe gerade eine zeitliche Verschiebung von Teilen der einkommensteuerlichen Bemessungsgrundlage erfordern. Eine Abmilderung dieses Konflikts in bezug auf die Kriterien "steuerliche Anerkennung von Verlusten" und "Berücksichtigung geminderter Zahlungsfähigkeit" wird allerdings durch das Verfahren der Steuerstundung erreicht, bei dem zwar nicht die Steuerbemessungsgrundlage, aber jedenfalls die Steuerschuld zeitlich verschoben wird. Zur Erfüllung des Kriteriums "Gewährleistung der Steuerfreiheit des Existenzminimums im Alter" sind pauschale Verfahren wie oben 56 dargestellt möglich, aller-

55 Vgl. Kapitel V.1.2.3.

56 Vgl. Kapitel V.1.2.1. 
dings bei erheblichem Verwaltungsaufwand und einem Verlust an Steuertransparenz. Aus Sicht einer Klassifizierung der Leibrentenversicherung als Ersparnis bleiben jedoch eine "periodengerechte Erfassung steuerlicher Leistungsfähigkeit" und eine "Umschichtung des steuerlichen Lebenseinkommens" grundsätzlich unvereinbar.

\section{(3) Leibrentenversicherung als Transferinstitution: Beiträge als Werbungskosten, Leistungen als Transfereinkommen}

Der wesentliche Unterschied zur traditionellen Sicht liegt darin, daß den Beitragszahlungen zu Leibrentenversicherungen kein Vermögensbildungscharakter eingeräumt wird. Den individuellen Beitragszahlungen liegen zwar eigennützige Motive (der Einkunftserzielung) zugrunde und der Beitragszahler erwirbt eine Gegenleistung in Form von Ansprüchen auf Leistungen bei Eintritt des Versicherungsfalles, diese Ansprüche stellen jedoch keine individuelle Vermögensposition dar 57 , da es sich lediglich um bedingte Ansprüche handelt, die für die Versicherten nicht disponibel und in ihren tatsächlichen späteren Auswirkungen auf die individuelle Einkommens- und Vermögensposition von exogenen Faktoren abhängig sind 58 .

Auch das Bundesverfassungsgericht läßt sich in seiner Rechtsprechung zu Anwartschaften auf Leibrenten der gesetzlichen Rentenversicherung, denen es einen Eigentumsschutz zuspricht, letztlich nicht

57 Anderer Auffassung, wenn auch z.T. mit auf gesamtwirtschaftliche Größen abstellenden Argumenten, sind Schmähl (1986, 109-111) und Birk (1986a, 18; 1986b, 137). Beachtlicherweise konstatiert aber auch Birk (1986b, 138, Fn.45) entscheidende Unterschiede zum Vermögen im allgemeinen.

58 Auch ganz geläufige Vermögenspositionen sind entweder nicht oder wenig disponibel (z.B. Kapitallebensversicherungen) oder mit Unsicherheiten behaftet (z.B. Optionsscheine), die spezielle Kombination aus beiden Eigenschaften ist aber gerade für Leibrentenversicherungen charakteristisch. Sie ist deshalb besonders schwerwiegend, weil sonst ein Mangel an Dispositionsfähigkeit durch Sicherheit der Anlage und ein Mangel an Sicherheit durch besondere Dispositionsfähigkeit kompensiert werden kann. 
als Anwalt einer Charakterisierung von Anwartschaften als Vermögen heranziehen. Es führt lediglich aus, daß die Regelgebundenheit der Rentenberechnung unter Berücksichtigung der Eigenleistung der Versicherten zu gewährleisten ist, womit aber keine individuelle Eigentumsgarantie für gezahlte Beiträge gegeben wird59. Insofern können Beiträge zum Erwerb von Anwartschaften auf Leibrenten nicht als Ersparnis gewertet werden.

Dies wäre anders, wenn es sich um marktfähige Rechtstitel handeln würde. Die Marktfähigkeit wird jedoch nicht nur durch rechtliche Rahmenbedingungen (insb. § 850(3b) ZPO), die wie alle Marktbeschränkungen ökonomischer Kritik ausgesetzt sind (Richter, 1987, 669-671), sondern auch durch in der Natur der Leibrentenversicherung liegende Faktoren, die sie für eine Gegenauslese (adverse selection) anfällig machen, eingeschränkt, weshalb auch bei reinen (privaten) Leibrentenversicherungen eine sonst bei Kündigung übliche Auszahlung des Deckungskapitals nicht vorgesehen ist ( $\$ 176$ VVG; Hagelschuer, 1983, 87f.). Entscheidend ist jedoch, daß das Ziel der Alterssicherung nur dann erreicht wird, wenn ein Ausstieg aus der Leibrentenversicherung über die Auszahlung bzw. Veräußerung von Ansprüchen ausgeschlossen wird. Die Funktionsfähigkeit der Alterssicherung verlangt gerade die Schaffung bzw. Erhaltung von institutionellen Regelungen, die eine Marktfähigkeit beschränken. Dies gilt umso mehr, wenn Aufwendungen zur Alterssicherung einkommensteuerlich eine Sonderstellung einnehmen.

Auch wenn es sich bei Ansprüchen auf Leibrenten also nicht um Vermögen handelt, so ist doch die Einkunftserzielungsabsicht beim Beitragszahler ebenso wie der Zusammenhang mit späteren Einkünften klar gegeben, so daß es naheliegt, die Beiträge als Werbungskosten für die Erzielung von in der Zukunft liegenden Einkünften zu behandeln60. Voraussetzung für die Anerkennung der Beiträge als Werbungskosten ist

$59 \mathrm{Vgl}$. Bundesverfassungsgericht $(1980 ; 1985 \mathrm{~b})$. Der vorgetragenen Interpretation zustimmend Krause (1982) und Ruland (1983, 207-210), z.T. anderer Auffassung ist Kaltenbach (1987, 72f.).

$60 \mathrm{Zu}$ den steuerrechtlichen Grundlagen vgl. Giloy (1979b) sowie U.Prinz (1988, Rz.162f.). 
allerdings die Steuerpflicht der damit erzielten Einnahmen (vgl. §§ 8 und 9 EStG). Insofern kann der Argumentation von Söhn (1985, 404), der ebenfalls für einen Werbungskostenabzug plädiert, nicht gefolgt werden, da dieser den Zusammenhang mit der Besteuerung lediglich der sog. Ertragsanteile nach $\S 22 \mathrm{Nr} .1$ EStG betont. Auch eine von Littmann (1983, 485) vorgetragene Werbungskosten- bzw. BetriebsausgabenBegründung kann nicht voll überzeugen, da dieser offensichtlich eine Zuordnung zu den im Alter gerade wegfallenden Erwerbseinkünften beabsichtigt, so daß hier bei Beachtung des kausalen Zusammenhangs die Werbungskosten-Definition ins Leere zielt.

Nach der Reinvermögenszugangstheorie, die am tatsächlichen Vermögenszugang und nicht an Erwartungswerte anknüpft, sind dann weder Beiträge zu Leibrentenversicherungen noch deren "Verzinsung" als Einkommen $\mathrm{zu}$ betrachten, allerdings ausgezahlte Renten in vollem Umfang, da erst im Zeitpunkt der Rentenzahlung individuell verfügbare Vermögenszugänge (und damit Einkommen) vorliegen 61 .

Eine solche steuersystematisch gebotene Regelung entspricht auch den übrigen Anforderungen an eine Besteuerung von Aufwendungen zur Alterssicherung. Durch eine Einordnung der Leibrentenversicherungsbeiträge als Werbungskosten wird sowohl der geminderten Zahlungsfähigkeit zum Zeitpunkt der Beitragszahlung Rechnung getragen als auch eine steuerliche Freistellung des Existenzminimums im Alter und eine Umschichtung des steuerlichen Lebenseinkommens erreicht. Der oben

61 Auf den ersten Blick scheint dies den Regelungen des Bewertungsgesetzes zu widersprechen, das sowohl noch nicht fällige Ansprüche aus Rentenversicherungen als auch laufende lebenslängliche Leistungen als Vermögen erfaßt ( $\S 12(4), 14$ BewG). Da kein Rückkaufswert vorhanden ist, bleiben noch nicht fällige Leibrentenversicherungen jedoch auch ohne Anwendung der Sondervorschriften in § 110(1) Nr.6 BewG ausgenommen (Rössler/ Troll, 1989, 156; Reuter, 1987, 221, 225). Fällige Ansprüche bleiben dann außer Betracht, wenn die Bedingungen für Alters- oder Berufsunfähigkeitsrenten vorliegen ( $\S 111 \mathrm{Nr} .3$ BewG). Differenzen verbleiben damit lediglich für Leibrenten, deren Empfänger weder das 60. Lebensjahr vollendet haben noch berufsunfähig sind. Eine erweiterte Anwendung des $\S 4$ BewG ("Aufschiebend bedingter Erwerb") könnte hier insgesamt Abhilfe schaffen. 
beschriebene Konflikt zwischen dem Ziel einer laufenden Erfassung steuerlicher Leistungsfähigkeit und den speziellen Zielen der Besteuerung von Alterseinkünften ist wegen der Klassifikation der Beiträge als Werbungskosten weitgehend gelöst. Die Behandlung von Beiträgen zu Leibrentenversicherungen als Werbungskosten ermöglicht eine steuersystematisch klare Einordnung. Auch die Eingrenzung auf diese eine Sicherungsform läßt sich schlüssig begründen. Es bedarf keiner Sonder- und Ausnahmeregelungen, wie das bei einer Klassifikation von Beiträgen als Ersparnis gemäß dem in der Literatur präferierten "Korrespondenzprinzip der nachgelagerten Art" der Fall wäre und auch bei der derzeitigen Zuordnung zu den "Sonderausgaben", die insgesamt den Charakter einer Verlegenheitslösung haben, der Fall ist. Bei einer Steuerpflicht für Rentenleistungen, wie sie hier vorgeschlagen wird, steht dem auch die Rechtsprechung des Bundesfinanzhofs (1986), der zu Recht Werbungskosten nur bei steuerpflichtigen Einkünften anerkennen will, entgegen anderslautender Äußerungen (Birk, 1986a, 18f.; Tipke/Lang, 1991, 377), nicht entgegen 62 .

Allerdings treten Übergangsprobleme bei einer entsprechenden Reform der derzeitigen steuerlichen Behandlung von Leistungen aus Leibrentenversicherungen auf. Diese ließen sich am besten durch pauschalierende Zuordnung von Rentenzahlungen zum Besteuerungsverfahren vor und nach einer Reform lösen (z.B. anhand der Zeitpunkte von Beitragszahlungen), so daß abrupte Änderungen und damit einhergehende Ungerechtigkeiten vermieden werden könnten.

Aus der Ex-ante-Sicht einer entscheidungsneutralen Besteuerung ergibt sich durch die einkommensteuerfreie Kapitalakkumulation der Institution Leibrentenversicherung ein "Renditevorteil" des Erwartungswerts einer Leibrentenversicherung im Vergleich zu anderen Anlagemöglichkeiten, so daß die Entscheidungsneutralität der Besteuerung verletzt wird. Dies ist eine unvermeidliche Folge der getroffenen Einkommenskonvention. Ähnliche Effekte ergeben sich bei einer ganzen Reihe anderer Betriebsausgaben bzw. Werbungskosten, z.B. bei einem

$62 \mathrm{Vgl}$. Bundesfinanzhof $(1986,749)$. Zu vorangegangenen höchstrichterlichen Entscheidungen vgl. Geiger (1987). 
Teil der nicht aktivierungsfähigen oder -pflichtigen Aufwendungen, Pensionsrückstellungen und beschleunigten Abschreibungen. Wünschenswert wird ein solcher Vorteil gar, wenn wegen der besseren Eignung von Leibrentenversicherungen zur Alterssicherung Nachteile auf anderen Gebieten (z.B. höhere administrative Kosten) ausgeglichen werden sollen oder wegen des meritorischen Charakters einer wirkungsvollen Alterssicherung63 eine Förderung der Leibrentenversicherung vertreten wird. Nachteilig ist dann lediglich, daß die Höhe der "Förderprämie" auf Vorsorgeaufwendungen von ca. 10-20\%64 nicht offen sichtbar und für alle gleich vom Gesetzgeber festgelegt wird, sondern sich aus dem Zusammenspiel von individuellem marginalem Steuersatz und dem Lebensalter im Zeitpunkt der Beitragszahlung ergibt65. Die Beträge, um die es hier geht, sind jedoch geringer als bei der sonst in diesem Zusammenhang angeführten Entlastung durch Steuerfreibeträge (zu der nach geltendem Recht die weitgehende Steuerfreiheit der Rentenleistungen hinzukommt), da es sich hier aus der oben beschriebenen Ex-ante-Sicht heraus lediglich um eine zeitliche Verschiebung von Steuerzahlungen und nicht um eine Steuerbefreiung handelt, außerdem nicht die gesamten Aufwendungen, sondern lediglich deren "Verzinsung" Grundlage des Steuervorteils sind.

\subsubsection{Gesetzliche Rentenversicherung}

Rentenleistungen der Gesetzlichen Rentenversicherung sind zukünftig im Regelfall ab dem 65. Lebensjahr vorgesehen, allerdings ist ein früherer oder späterer Renteneintritt verbunden mit versicherungsmathematisch berechneten Ab- bzw. Zuschlägen möglich. Neben dem Altersrisiko wird auch dem Berufs- und Erwerbsunfähigkeitsrisiko durch entsprechende

63 Vgl. Kapitel V.1.2.3.

$64 \mathrm{Vgl}$. die Berechnungen unter (2e) in diesem Kapitel.

65 Dabei sinkt die errechnete "Prämienhöhe" durch im Zeitablauf real ansteigende Einkommen; aufgrund der Abhängigkeit vom individuellen Steuersatz mögliche regressive Verteilungswirkungen werden durch die üblicherweise längeren Ausbildungszeiten von Versicherten mit höheren Einkommen reduziert (Verminderung der Zinseszinseffekte). 
Rentenzahlungen Rechnung getragen. Im Todesfall des Versicherten werden ggf. Hinterbliebenenrenten an dessen Ehegatten und Kinder gezahlt 66 .

Eine Versicherungspflicht besteht nahezu für alle Arbeitnehmer und einige selbständig Tätige, andere Personengruppen können sich i.d.R. freiwillig versichern. Die gesetzliche Rentenversicherung finanziert sich vor allem über laufende Beitragszahlungen ihrer Mitglieder, die i.d.R. als Prozentsatz der Bruttolohn- und -gehaltssumme (bis zur Beitragsbemessungsgrenze von im Jahr 1993 7.200 DM monatlich) berechnet werden. Daneben zahlt der Bund einen Bundeszuschuß. Die eingenommenen Beiträge werden - abgesehen von einer geringfügigen Rücklage - jeweils direkt zur Finanzierung der laufenden Rentenzahlungen verwendet (Umlageverfahren).

Die Rentenberechnung erfolgt grundsätzlich beitragsbezogen, jedoch gibt es einige Abweichungen vom versicherungstechnischen Äquivalenzprinzip, insbesondere die beitragsfreie Hinterbliebenensicherung67, die Anrechnung beitragsfreier Zeiten (z.B. Ausfallzeiten) und die Höherbewertung von Beitragszeiten (z.B. für die ersten fünf Versicherungsjahre und bei der Rente nach Mindesteinkommen). Hinzu kommen Abweichungen vom Äquivalenzprinzip, die auf das Umlageverfahren zurückzuführen sind, und damit die Rentenanpassungen von der Entwicklung der Relation Beitragszahler zu Rentnern abhängig machen. Die geschlechtsspezifische Beitragsdifferenzierung, wie sie in der privaten Leibrentenversicherung üblich ist, ist dagegen nicht notwendigerweise Bestandteil einer versiche-

66 Lediglich in diesem Bereich gibt es auch in der gesetzlichen Rentenversicherung Zeitrenten (vgl. § 46(2) und § 48(4) SGB VI): Die Waisenrenten werden "nur" bis zum 18. bzw. 25. Lebensjahr gezahlt, ebenfalls wird unter Umständen auch die sog. große Witwen- oder Witwerrente auf die Zeit der Kindererziehung (bis zum 18. Lebensjahr des Kindes) beschränkt.

67 Die Hinterbliebenensicherung läßt sich durchaus auch als Bestandteil des Versicherungsvertrags verstehen. Damit wird jedoch das "System auf den verheirateten Versicherten hin angelegt" (Kolb, 1985, 130), Ledige werden zu Benachteiligten ihrer Zwangsversicherung, da sie keine Ausweichmöglichkeiten haben. Vgl. für die Beibehaltung einer obligatorischen Hinterbliebenensicherung Kolb (1985, 130f.), sowie anderer Auffassung Krupp (1987, 8f.) und MIT-Rentenkommission (1988, 136f.). 
rungsäquivalenten Lösung68. Die Höhe der bei Renteneintritt berechneten Rente wird jährlich entsprechend der Entwicklung der Nettolöhne und -gehälter angepaßt (Dynamisierung) ${ }^{69}$.

Die gesetzliche Rentenversicherung beruht wie auch die private Leibrentenversicherung auf der Grundkonzeption der Leibrentenversicherung 70 , beide werden auch nach dem derzeit gültigen Einkommensteuerrecht gleich behandelt 71 . Allerdings ist $\mathrm{zu}$ prüfen, ob die institutionellen Besonderheiten der gesetzlichen Rentenversicherung Konsequenzen für die einkommensteuerliche Behandlung haben sollten.

Bei der gesetzlichen Rentenversicherung handelt es sich für den größten Teil der versicherten Personen um eine Zwangsversicherung. Die Versicherten sind durch die abzuführenden Zwangsbeiträge in der Verwendung ihres Einkommens eingeschränkt. Nach der traditionellen Sichtweise (Beiträge als Ersparnis) spielt die Reduzierung des frei

68 Dies erscheint zunächst mit Blick auf die unterschiedlichen Lebenserwartungen von Männern und Frauen sowie die Praxis der privaten Versicherer als nicht sachgerecht. Wenn aber berücksichtigt wird, daß die Lebenserwartung mit dem Ergebnis ähnlich hoher quantitativer Unterschiede auch nach anderen Kriterien differenziert ermittelt werden kann, z.B. nach dem Beruf, dem Bildungsstand, dem Einkommen oder nach Regionen, so ist es zwar verständlich, daß sich Versicherungsunternehmen bei ihrer Beitragsgestaltung auf das leicht feststellbare Merkmal Geschlecht beschränken, im Hinblick auf die Willkür der Auswahl dieses einen Merkmals ist es aber gleichwohl gerechtfertigt, in der gesetzlichen Rentenversicherung auf eine Beitragsdifferenzierung in bezug auf unterschiedliche gruppenspezifische Lebenserwartungen ganz zu verzichten. Beachtenswert ist auch, daß eine Gegenauslese (adverse selection) in einer Zwangsversicherung nicht möglich ist. Vgl. Wagner (1985a, 175-178; 1985b, 192f.) sowie Wolff (1988, 165f.).

69 Rein formal bildet die Entwicklung der Nettolöhne und -gehälter erst ab Inkrafttreten der Rentenreform '92 die Grundlage für die Dynamisierung der Renten der gesetzlichen Rentenversicherung, faktisch war aber auch in der Vergangenheit schon diese Relation ausschlaggebend, wozu allerdings diskretionäre Maßnahmen sowie die Einführung eines Krankenversicherungsbeitrags der Rentner notwendig waren.

$70 \mathrm{Zu}$ Gemeinsamkeiten und Unterschieden vgl. Kreßmann (1971) und Meinhold (1985, 13-26).

71 Vgl. Kapitel V.1.3.4.1 (2c). 
verfügbaren Einkommens durch die Beitragszahlung eine große Rolle ${ }^{72}$, wenn auch von der Einschränkung der aktuellen steuerlichen Zahlungsfähigkeit nicht unbedingt und in vollem Umfang auf eine geminderte steuerliche Leistungsfähigkeit geschlossen werden kann (Andel, 1980, 373f.; Haller, 1981a, 60). Werden dagegen Beiträge zu Leibrentenversicherungen als Werbungskosten angesehen, dann ist dem Umstand der Verminderung des verfügbaren Einkommens bereits Rechnung getragen, eine weitergehende Sonderbehandlung aus diesem Grunde ist weder angemessen noch erforderlich 73 .

Der Umstand des Versicherungszwangs in der gesetzlichen Rentenversicherung rechtfertigt jedoch an sich keine besondere Förderung 74 , jedenfalls wenn dem Argument einer verminderten Steuerzahlungsfähigkeit bereits Rechnung getragen ist. Der richtige Ansatzpunkt für eine Gleichstellung aller Steuerpflichtigen scheint hier eher in der Regelung der Versicherungspflicht zu liegen ${ }^{75}$, zumal diese ebenso wie steuerliche Maßnahmen im Entscheidungsbereich des Gesetzgebers liegt und damit gleichzeitig den meritorischen Aspekten einer Mindestsicherung Rechnung getragen werden kann.

Eine weitere Besonderheit der gesetzlichen Rentenversicherung sind Umverteilungselemente, die z.T. an die Versichertenbiografie anknüpfen, z.T. von den globalen Rahmenbedingungen eines Umlageverfahrens abhängen ${ }^{76}$. Aus steuersystematischer Sicht sind Beiträge, die der Umverteilung dienen, wie zusätzliche Steuern auf die Bemessungsgrundlage Einkommen zu behandeln. Es sollte daher für die betreffenden Beitragsteile ein Abzug von der Bemessungsgrundlage der Einkommensteuer

72 Vgl. Albers $(1980,199)$, Tipke $(1980,5)$ sowie Söhn $(1985,406 ; 1986$, 331).

73 Vgl. Kapitel III.3.3.

74 Zumal für alle Nicht-Pflichtversicherten ebenfalls auf einen faktischen Zwang zur Altersvorsorge verwiesen werden kann.

$75 \mathrm{Zu}$ denken ist hier insbesondere an eine Versicherungspflicht für alle Bürger (Krupp, 1987).

$76 \mathrm{Zu}$ Umverteilungswirkungen der gesetzlichen Rentenversicherung vgl. Wagner (1985b) sowie Rehfeld/Luckert (1989, 45-47). 
zugelassen werden 77. Da dies ohnehin vorgesehen ist (Beiträge als Werbungskosten!), treten hierdurch keine verfahrenstechnischen Probleme auf 78 .

Es stellt sich weiter die Frage nach einer Begünstigung der gesetzlichen Rentenversicherung, da diese sich durch interpersonelle und eventuelle intergenerationale Umverteilungen jedenfalls für einen erheblichen Teil der Mitgliederschaft als weniger vorteilhaft als eine private Leibrentenversicherung erweist. Die beste Lösung wäre jedoch eine weitgehende Trennung der Ziele Alterssicherung und Umverteilung79, wodurch einerseits Intransparenzen des Alterssicherungssystems 80 vermieden würden, andererseits die Finanzierung der über die versicherungsimmanente Umverteilung hinausgehenden Umverteilungsmaßnahmen nicht nur von den Versicherten der gesetzlichen Rentenversicherung aufzubringen wäre. Daher sollten interpersonelle Umverteilungen über den Bundeshaushalt finanziert und intergenerative Umverteilungen durch den Bundeshaushalt oder über eine Veränderung von Rücklagen der gesetzlichen Rentenversicherung neutralisiert werden. Allgemeine steuerliche oder außersteuerliche Fördermaßnahmen wegen dieser Umverteilungseffekte in der gesetzlichen Rentenversicherung sind aufgrund der damit verbundenen Intransparenz, der möglichen Kumulation von Begün-

77 Vgl. Kapitel III.3.2.

78 Es läßt sich zwar darüber streiten, welcher Anteil der Beiträge als Werbungskosten und welcher zur Vermeidung einer Doppelbesteuerung abzugsfähig ist. Dies bleibt aber letztlich ohne Folgen, da beides zu einem Abzug von der Bemessungsgrundlage führt. Im Rahmen des Modells "Beiträge als Ersparnis" könnten dagegen erhebliche Probleme auftreten.

79 Auch wenn grundsätzlich Einigkeit besteht, nach dem versicherungstechnischen Äquivalenzprinzip vorzugehen, bleiben noch Ermessensspielräume z.B. bezüglich der Separierung gruppenspezifischer Risiken. Vgl. Krupp (1988, 498-502), Wagner (1985a, 175-178), sowie aus versicherungstheoretischer Sicht Eisen (1988).

80 Diese werden durch eine Vielzahl von Einzelmaßnahmen verursacht, welche zudem noch zu Moral hazard führen können und in ihrer Summe eher unerwünschte Verteilungseffekte bewirken. Zusammenfassend hierzu Wagner (1984, 303-308; 1985b). Zur Richtung des Gesamteffekts vgl. auch die Ergebnisse der neueren Untersuchung von Rehfeld/Luckert $(1989,56)$. 
stigungen (für Umverteilungsgewinner) und des höheren fiskalischen Aufwands hier regelmäßig die schlechtere Lösung.

Die Besteuerung der Leistungen der gesetzlichen Rentenversicherung wirft keine besonderen Probleme auf, da hier die tatsächliche steuerliche Leistungsfähigkeit unter Berücksichtigung aller Umverteilungsvorgänge Gegenstand ist und damit eine volle Besteuerung der Leistungen die angemessene Lösung darstellt.

\subsubsection{Private Leibrentenversicherung}

Wegen der in der privaten Leibrentenversicherung im Vordergrund stehenden versicherungsäquivalenten Ausgestaltung bildet diese das Grundmodell der Leibrentenversicherung besonders gut ab. Der Charakter von Versicherungsbeiträgen wird hier nicht durch Ex-ante-Umverteilungen, wie das zum Teil in der gesetzlichen Rentenversicherung der Fall ist, verfälscht. Eine risikoäquivalente Beitragsdifferenzierung berücksichtigt beispielsweise die unterschiedlichen geschlechtsspezifischen Lebenserwartungen 81 , auch ist der Einschluß einer Hinterbliebenenversorgung in der privaten Leibrentenversicherung zwar möglich, erfordert aber einen erhöhten Beitrag entsprechend dem für den Versicherer zusätzlich entstehenden Risiko. Eine Dynamisierung der Rente erfolgt hier unter Umständen aufgrund einer besonderen Ausgestaltung der Versicherung oder über die Verwendung der Überschußanteile.

Besondere Probleme einer einkommensteuerlichen Behandlung von privaten Leibrentenversicherungen ergeben sich durch die Vielzahl von Variationsmöglichkeiten des Grundmodells, die in diesem Bereich anzutreffen sind. Aufgrund der gegebenen Vertragsfreiheit kommen z.B. die Vereinbarung eines Wahlrechts zum Zeitpunkt des Rentenbeginns zwischen der Auszahlung einer Kapitalsumme und Rentenzahlung, Vereinbarungen über Beitragsrückerstattungen und Mindestrentenlaufzeiten für den Todesfall des Versicherten vor. Solche Abweichungen vom Grund-

81 Zur Kritik an einer solchen Vorgehensweise - insb. im Rahmen einer Zwangsversicherung - vgl. die Ausführungen im vorangegangenen Abschnitt. 
modell der Leibrentenversicherung verändern den Charakter der Versicherung in Richtung einer Kapitalsummenversicherung bzw. allgemeiner Vermögensbildung. Bis zu einer definitiven Festlegung auf eine reine Leibrentenversicherung sind solche Versicherungsverträge daher so wie die ihnen am nächsten stehenden Versicherungsarten zu behandeln, was i.d.R. eine laufende einkommensteuerliche Erfassung der sich bei Gegenüberstellung von Veränderungen des Rückkaufswerts und der gezahlten Beiträge ergebenden Wertzuwächse beinhaltet ${ }^{82}$.

\subsubsection{Beamtenversorgung}

Die Beamtenversorgung ist zwar rechtlich Teil der "hergebrachten Grundsätze des Berufsbeamtentums", von der Sache her handelt es sich jedoch ebenfalls um ein Rentenversicherungssystem (Ruland, 1983, Rz.42-45,50). Beamte erwerben den Anspruch auf eine Leibrente allerdings nicht gegenüber einem gesonderten Rentenversicherungsträger, sondern gegenüber dem bisherigen staatlichen Arbeitgeber. Da kein institutionell eigenständiger Rentenversicherungsträger existiert, werden auch keine Rentenversicherungsbeiträge abgeführt 83 - die Wirkung der Beamtenversorgung ist jedoch genauso bindend wie eine durch explizite Beitragszahlungen erworbene Anwartschaft z.B. in der gesetzlichen Rentenversicherung. Dies wird nicht zuletzt durch die Pflicht des staatlichen Arbeitgebers zur Nachentrichtung von Beiträgen zur gesetzlichen Rentenversicherung bei Ausscheiden von Beamten aus dem Dienst deutlich84. Es könnte daher daran gedacht werden, entsprechend dem Vorgehen in der Volkswirtschaftlichen Gesamtrechnung (Stobbe, 1989, 259),

82 Vgl. die Ausführungen in Kapitel V.1.3.2.

83 Gleichwohl kann der Erwerb von Ansprüchen auf Altersversorgung als Teil des Gehalts interpretiert werden (Ruland, 1983, Rz.50; Siebeck, 1989, 38f., 145).

84 Allerdings ist auch der "Eigentumsschutz" im wesentlichen auf die Höhe der Nachversicherungspflicht beschränkt, die vor allem aufgrund der unterschiedlichen Bestimmungen zum beitragspflichtigen Entgelt, zur Beitragsbemessungsgrenze und zur "Rentenberechnung" regelmäßig unter dem Versorgungsanspruch in der Beamtenversorgung liegt (vgl. § 181 SGB VI; Ruland, 1983, Rz. 67-70, 113-119, 511). 
bei Beamten einen fiktiven Beitrag zu ihrer Alterssicherung auf der Gehaltsabrechnung auszuweisen, der dann auch nach derzeitigem Recht (Beiträge als Ersparnis!) Bestandteil des steuerlichen Einkommens würde. In der Praxis würden jedoch wegen der Nichtlinearitäten im System der Beamtenversorgung 85 erhebliche Probleme entstehen. Werden dagegen auch hier die Beiträge als Werbungskosten von der Bemessungsgrundlage abzugsfähig, ist es einkommensteuerlich unerheblich, wenn ihre exakte Höhe unbekannt ist, da sich Erhöhungs- und Abzugsbetrag definitionsgemäß gerade entsprechen. Die zufließenden Renten (Pensionen) sind dann auch hier wieder in vollem Umfang einkommensteuerpflichtig. Nach der derzeitigen einkommensteuerlichen Regelung werden fiktive Beiträge zur Beamtenversorgung steuerlich nicht erfaßt, so daß in diesem Bereich eine faktische Übereinstimmung mit der allgemein für Leibrentenversicherungen vorgeschlagenen Lösung besteht. Gleichwohl gelten jedoch für Beamte die gleichen Höchstbeträge für Vorsorgeaufwendungen, was dieser Personengruppe im Vergleich zu allen anderen Steuerpflichtigen zusätzliche Spielräume für steuerbegünstigtes Sparen oder steuerbegünstigte Vorsorge verschafft. Auch die einkommensteuerliche Erfassung der Leistungen aus der Beamtenversorgung entspricht im Grundsatz der hier vorgeschlagenen Regelung. Es wird jedoch ein zusätzlicher Versorgungsfreibetrag nach $\S 19(2)$ EStG in Höhe von $40 \%$ der Versorgungsbezüge, maximal $6000 \mathrm{DM}$, eingeräumt, für den eine überzeugende steuersystematische Begründung fehlt ${ }^{86}$. Wegen der Zuordnung der Versorgungsbezüge zu den Einkünften aus nichtselbständiger Arbeit ( $§ 19(1)$ EStG) kommt noch die Arbeitnehmer-Pauschale von 2000 DM hinzu ( $\$ 9 \mathrm{a}$ EStG), außerdem die Vorsorgepauschale nach $\S 10 \mathrm{c}(3) \mathrm{EStG}$ in Höhe von

85 Im Gegensatz zur gesetzlichen Rentenversicherung, wo - vereinfacht ausgedrückt - das Durchschnittsgehalt während der Erwerbsphase als Grundlage der Rentenberechnung dient, außerdem die einzelnen Beitragsjahre gleich gewichtet werden, wird in der Beamtenversorgung vom letzten Gehalt ausgegangen und mit im Zeitablauf abnehmenden Steigerungssätzen gerechnet.

86 Allerdings läßt sich der Versorgungsfreibetrag als Folgebegünstigung der zum Zeitpunkt der Einführung bereits bestehenden Begünstigung der Leibrentenbezüge interpretieren. 
$18 \%$ des Arbeitslohns, maximal $2000 \mathrm{DM}$, die beide in dieser Höhe sachlich nicht zu rechtfertigen sind.

\subsubsection{Betriebliche Altersversorgung}

In der betrieblichen Altersversorgung sind bezüglich der Organisationsform Direktzusagen, Unterstützungskassen, Direktversicherungen sowie Pensionskassen zu unterscheiden. Auch wenn bei Unterstützungskassen Zeitrenten vorkommen und bei Direktversicherungen Kapitalversicherungen möglich sind, so handelt es sich doch i.d.R. um Leibrentenversicherungen 87 .

Ob die Beiträge des Arbeitgebers nach geltendem Recht beim Arbeitnehmer als Einkommensbestandteil ausgewiesen werden, hängt von der Organisationsform der betrieblichen Altersversorgung ab. Diese steuerliche Differenzierung kann immer weniger mit vorhandenen Unterschieden in der Rechtsposition des Arbeitnehmers begründet werden, da faktisch inzwischen auch bei Direktzusagen und Unterstützungskassen ein gesicherter Anspruch besteht (v.Maydell, 1983, 266-269; H.Schwab, 1988, 51, 64). Daher gilt auch hier, daß Beiträge des Arbeitgebers individuell als Lohnbestandteil zugerechnet werden sollten, beim Arbeitnehmer allerdings als Werbungskosten (Leibrentenversicherung!) abzuziehen sind. Ausgezahlte Renten sind in vollem Umfang in die Steuerpflicht einzubeziehen.

Für die hier vor allem interessierende Frage der Besteuerung der Arbeitnehmer treten damit wiederum keine Probleme auf. Steuerrechtliche Abgrenzungen der "Beitragshöhe" - wie sie auch im geltenden Recht zu finden sind - sind vor allem zur Vermeidung einer Steuerumgehung durch den Arbeitgeber nötig, sofern ihm ein direkter Zugriff auf die für Zwecke der Altersversorgung seiner Arbeitnehmer ausgewiesenen Mittel wie bei der Direktzusage und der Unterstützungskasse verbleibt. Ein

87 Für die übrigen genannten Fälle kann auf die entsprechenden Kapitel verwiesen werden. Zur relativen Häufigkeit von Kapitalversicherungen auf der einen, (Leib-)Rentenversicherungen auf der anderen Seite vgl. v.Maydell $(1983,263)$. 
Mißbrauch wird aber bereits durch individuelle Rechtsansprüche der Arbeitnehmer weitgehend verhindert, lediglich die Kalkulationsgrundlagen der "Versicherung" sind im einzelnen zu prüfen bzw. vorzugeben. Die Aufwendungen des Arbeitgebers könnten selbst bei einer steuerlichen Behandlung der "Beiträge" als Ersparnis als Grundlage für eine individuelle Zurechnung dienen, da die Kalkulation des Arbeitgebers auf den individuellen Daten aufbaut (H.Schwab, 1988, 57).

Die derzeitigen steuerlichen Regelungen zur betrieblichen Altersversorgung 88 sehen beim Arbeitgeber durchgängig die Abzugsfähigkeit der Aufwendungen von der Bemessungsgrundlage als Betriebsausgabe (direkt oder über die Zuführung zu Rückstellungen) vor, zum Teil unter Vorgabe von Berechnungsmodalitäten und Höchstwerten ( $\S 4 b-d, 6 a$ EStG). Beim Arbeitnehmer ist dagegen die Behandlung der einzelnen Formen der betrieblichen Altersversorgung trotz ihrer Ähnlichkeit so unterschiedlich, daß Kritik an den Regelungen geradezu provoziert wird (Henke, 1988, 269f.): Die Leistungen aufgrund von Direktzusagen und von Unterstützungskassen werden wie Beamtenpensionen behandelt, die Leistungen von Direktversicherungen und Pensionskassen werden dagegen als Leibrentenzahlungen eingestuft. Daher besteht für Direktzusagen und die Beiträge an Unterstützungskassen keine Steuerpflicht, während Beiträge zu Direktversicherungen und Pensionskassen als Teil des Arbeitslohns steuerpflichtig sind, allerdings nach Abzug eines zusätzlichen sogenannten Zukunftssicherungsfreibetrages nach $\S 2$ 2(2) $\mathrm{Nr}$.3 LStDV in Höhe von 312 DM pro Jahr und der Möglichkeit einer Pauschalbesteuerung mit einem Steuersatz von $15 \%$ bis zu einem Betrag von $3000 \mathrm{DM}$ pro Jahr ( $\$ 40 \mathrm{~b} \mathrm{EStG}$ ). Die Rentenzahlungen sind bei den Gestaltungsformen der Direktzusage und der Unterstützungskasse grundsätzlich steuerpflichtig, allerdings nach Abzug des Versorgungsfreibetrags von $40 \%$, max. $6000 \mathrm{DM}$; lediglich für Leistungen aus Unterstützungskassen gibt es unter bestimmten Umständen zusätzlich die Möglichkeit einer Pauschalbesteuerung analog zu § 40a EStG (H.Schwab, 1988, 71).

88 Henke (1988, 269-272) und H.Schwab (1988, 60-63, 65-68, 70f., 77-82, 85-87) geben einen guten Überblick über die Grundlinien, der allerdings nicht mehr auf dem aktuellsten Stand ist. 
Die Leistungen aus Direktversicherungen und Pensionskassen unterliegen dagegen der Ertragsanteilsbesteuerung nach $\S 22 \mathrm{Nr} .1 \mathrm{EStG}$, was damit zusammenhängt, daß in diesen Fällen bereits die Beiträge der individuellen Vermögenssphäre zugeordnet werden.

\subsubsection{Leibrentenvereinbarungen unter Privaten}

Es sind noch all jene Leibrentenvereinbarungen zu behandeln, die weder über spezielle Versicherungsinstitutionen noch durch den Arbeitgeber nach den besonderen Bestimmungen des Betriebsrentengesetzes abgewickelt werden. In aller Regel erfolgen solche Zusagen als Gegenleistung für die Übertragung eines Vermögensobjekts an den Verpflichteten. Die Hingabe eines Vermögensgutes ist der Beitragszahlung an ein Versicherungsunternehmen vergleichbar, daher ist eine identische einkommensteuerliche Behandlung angebracht ${ }^{89}$, also der Abzug des Vermögenswertes von der Bemessungsgrundlage der Einkommensteuer als Werbungskosten. Die Leibrentenzahlungen sollten dann wiederum voll einkommensteuerpflichtig sein.

Beim zur Zahlung einer Leibrente Verpflichteten ist die Anwendung des Vermögensvergleichs zur Einkünfteermittlung hier das adäquate Verfahren. Allein aus Praktikabilitätsgründen ist jedoch auch die Anwendung einer Einnahmenüberschußrechnung denkbar (Tipke/Lang, 1991, 237f.).

Bei der Einnahmenüberschußrechnung werden Einkünfte in Höhe des erworbenen Vermögenswertes angesetzt, die Leibrentenzahlungen jeweils als Einkunftserzielungskosten berücksichtigt. Damit wird ein sehr einfaches Verfahren angewandt, womit aber auch ein in Anbetracht der eingegangenen Leibrentenverpflichtung zu starker steuerlicher Zugriff in

89 Das bedeutet nicht, private Leibrentenverpflichtungen und Leibrentenversicherungen seien beliebig austauschbar, da i.d.R. spezielle Motive wie die gleichzeitige Einräumung eines Nutzungsrechts des übertragenen Vermögensgutes für die Wahl einer privaten Leibrentenvereinbarung sprechen. Aus steuerlicher Sicht liegen jedoch - gegebenenfalls unter Berücksichtigung von Nebenvereinbarungen - grundsätzlich vergleichbare Tatbestände vor. 
der Basisperiode verbunden ist, der erst nach und nach korrigiert werden könnte. Für die Steuerpflichtigen bliebe ein Liquiditätsnachteil und ein tendenzieller Nachteil durch eine zu starke Steuerprogression bestehen 90 . Bei "Bilanzierung" des erworbenen Vermögensgutes einerseits, von Rückstellungen für erwartete Leibrentenzahlungen andererseits, steigt Jahr für Jahr der "Eigenkapitalanteil" am Vermögenserwerb an. Einkommensteuerlich zu berücksichtigen ist dann jeweils die Differenz aus der Reduktion der erwarteten Rentenzahlungen im Vergleich zur Vorperiode ("Vermögenszugang") und den Rentenzahlungen der Periode. Während der Rentenlaufzeit ist entsprechend mit einer Minderung der Steuerbemessungsgrundlage zu rechnen, bei Beendigung der Rentenlaufzeit durch Tod des Rentenberechtigten mit einer Erhöhung (in Höhe des Restbuchwerts des "Fremdkapitalanteils"). Hier ist die Vermögenssituation durch die Berücksichtigung von "Rückstellungen" realistischer dargestellt ${ }^{91}$; tatsächliche Gewinne und Verluste werden letztendlich wie beim ersten Verfahren erfaßt, allerdings unter Vermeidung des Liquiditäts- und Reduzierung des Progressionsnachteils.

Problematisch sind beide Verfahren dann, wenn die Erträge des übertragenen Vermögensgutes, etwa weil sie in einer konsumtiven Nutzung bestehen, einkommensteuerlich nicht erfaßt werden. Denn die als Werbungskosten angesetzten Rentenzahlungen beinhalten auch eine Verzinsung des eingesetzten Kapitals. Im Vergleich zur einzig denkbaren Alternative, der einkommensteuerlichen Nichtbeachtung von privaten Leibrentenvereinbarungen durch Zuordnung zur "privaten Vermögens-

90 Ein Zinsnachteil besteht hier für die Steuerpflichtigen nicht, da sich der Wert des Vermögensgutes als abdiskontierter Wert der erwarteten zukünftigen Leibrentenzahlungen, die dann ja als Werbungskosten zu berücksichtigen sind, errechnet.

91 Daß hier der Erwartungswert der Leibrentenverpflichtung "bilanziert" werden soll, ließe sich sicher noch weiter diskutieren. Unter Rückgriff auf den eher abwartenden Charakter einer Ex-post-Besteuerung und eine unter Kaufleuten übliche "vorsichtige" Bewertung läßt sich diese Lösung allerdings vertreten. Unproblematisch ist eine solche Bewertung erst, wenn durch eine Vielzahl von Leibrentenverpflichtungen - wie bei Versicherungsunternehmen - die Gesamtsumme der Verpflichtungen über einen Risikoausgleich recht genau bestimmt werden kann. 
sphäre", wie im wesentlichen derzeit praktiziert ${ }^{92}$, ist dieser Nachteil jedoch vernachlässigbar, da sonst die betragsmäßig weit höheren Gewinne und Verluste durch vom Durchschnitt abweichende Lebensdauer der Leibrentenberechtigten einkommensteuerlich nicht berücksichtigt werden könnten.

\subsubsection{Versicherungen zugunsten Dritter}

Die einkommensteuerliche Behandlung von Versicherungen zugunsten Dritter ist, sofern es sich nicht um Leistungsentgelte handelt ${ }^{93}$, im Zusammenhang mit anderen Zuwendungen zugunsten Dritter zu erörtern ${ }^{94}$. Da solche Versicherungen jedoch oft direkt an Versicherungen zur eigenen Altersvorsorge gekoppelt sind, soll an dieser Stelle bereits eine Charakterisierung dieser Verträge und eine grobe Skizzierung von möglichen Lösungsansätzen ihrer einkommensteuerlichen Behandlung vorgenommen werden.

Bei Versicherungsverträgen, die - ausschließlich oder nur unter bestimmten Umständen - für den Eintritt des Versicherungsfalles Dritte als Begünstigte vorsehen, handelt es sich grundsätzlich um Schenkungen, also um Transfers, die lediglich in Form von Versicherungen erfolgen. Ihre einkommensteuerliche Behandlung sollte daher derjenigen von direkten Zuwendungen an Dritte folgen.

Die Versicherungsbeiträge sind vom Transfergeber, dem Beitragszahler, als Einkommensverwendung zugunsten Dritter im Grundsatz aus versteuertem Einkommen $\mathrm{zu}$ leisten. Beim Transferempfänger fallen steuerpflichtige Einkünfte an, die allerdings gleichzeitig wie eigene Ver-

92 Vgl. §§ 9(1) Nr.1, 22 Nr.1 EStG sowie Tipke/Lang (1991, 364f.). Bei einer umfassenden Einkommensbesteuerung, von der in dieser Arbeit ausgegangen wird, ist diese Möglichkeit jedoch wegen der Erfassung von Wertveränderungen auch im Privatvermögen ohnehin ausgeschlossen (vgl. Tipke/Lang, 1991, 369f.).

93 Das ist i.d.R. bei Arbeitgeberleistungen zugunsten von Arbeitnehmern der Fall.

94 Vgl. Kapitel VI.6. 
sicherungsbeiträge zu behandeln, d.h. unter Umständen als Werbungskosten steuerlich absetzbar sind.

Eine Übertragung dieser Grundsätze auf die wichtigsten in diesem Zusammenhang anzutreffenden Versicherungsarten ergibt folgendes:

Arbeitgeberleistungen zugunsten von Arbeitnehmern stellen in aller Regel einen Teil des Leistungsentgelts dar; um Mißverständnisse auszuschließen, sollen sie hier gleichwohl nochmals explizit angesprochen werden. Beim Arbeitgeber liegen damit Betriebsausgaben vor, beim begünstigten Arbeitnehmer steuerpflichtige Einkünfte. Sofern es sich allerdings um Beiträge zu Leibrentenversicherungen handelt, sind in gleicher Höhe Werbungskosten des Arbeitnehmers für die Erzielung zukünftiger Renteneinkünfte anzusetzen, womit sich per Saldo eine Steuerfreiheit dieser Beiträge ergibt.

Von großer Bedeutung sind Leibrentenversicherungen, die, wie z.B. die gesetzliche Rentenversicherung, für den Fall des Todes des Versicherten eine Hinterbliebenensicherung vorsehen. Der bisherigen Argumentation folgend müßte eine steuersystematische Lösung den Abzug von Beiträgen für die eigene Person als Werbungskosten ermöglichen, während Beiträge zugunsten Dritter grundsätzlich - auf Ausnahmen wird später zurückzukommen sein - steuerpflichtig wären. Die Voraussetzung hierfür ist allerdings, daß der Beitrag zur Altersversicherung in zwei Teilbeträge aufgeteilt werden kann, einen Beitrag für die eigene Person und einen Beitrag für eine Hinterbliebenensicherung.

Rein technisch dürfte eine solche Fiktion von zwei Versicherungsverträgen einfach durchzuführen sein, da die Kalkulationsgrundlagen vorliegen und ein privates Versicherungsunternehmen auch auf dieser Grundlage eine "Versicherung auf verbundene Leben" (Schwebler, 1988, 419) kalkulieren würde. So gesehen bedürfte es nur einer Bestimmung im Einkommensteuergesetz, wonach ein Abzug von Rentenversicherungsbeiträgen vom getrennten Ausweis von Beiträgen für die eigene und die Hinterbliebenensicherung abhängig gemacht wird, um diesen Lösungsansatz in der Praxis zu verwirklichen.

In der gesetzlichen Rentenversicherung wird jedoch ein Beitrag für das Risiko der Hinterbliebenensicherung nicht nach versicherungs- 
mathematischen Grundsätzen, dem versicherungstechnischen Äquivalenzprinzip, kalkuliert. Statt, um den Fall der Ehegattensicherung herauszugreifen95, die Beiträge nach dem Familienstand zu differenzieren und die (Hinterbliebenen-)Leistungen ins Verhältnis zur Ehedauer (Beitragsdauer) zu setzen, wie es dem Äquivalenzprinzip entsprechen würde, werden Hinterbliebenenleistungen unabhängig von der Ehedauer in Relation zur Rente des Verstorbenen gezahlt. Damit wird die bewußte Entscheidung der Versicherten zur Eheschließung als stochastische Größe angenommen 96 , Zeiten vor der Eheschließung werden beim Hinterbliebenen, nicht jedoch beim Verstorbenen, völlig ignoriert. Bei Wiederheirat wird die Hinterbliebenenrente für die Dauer der neuen Ehe ausgesetzt. Eine steuersystematisch einwandfreie Behandlung der derzeit existierenden Verträge ist bei dieser Art der Ausgestaltung unmöglich: Eine volle steuerliche Berücksichtigung der Beiträge von Ledigen als Beiträge für die eigene Alterssicherung 97 würde die Möglichkeit einer späteren Heirat unberücksichtigt lassen, die Einordnung eines Beitragsteiles für Hinterbliebenensicherung für Verheiratete die Möglichkeit einer späteren Scheidung ebenso ausschließen wie diejenige einer Wiederheirat nach dem Tod eines der Ehepartner.

Bei der verbreitetsten Form der privaten Lebensversicherung, der Versicherung auf den Erlebens- und Todesfall, handelt es sich in der Regel - die Erlebensfalleistung ist an den Versicherten zu zahlen, die

95 Auf Waisenrenten, also die Hinterbliebenensicherung für Kinder, soll hier nicht näher eingegangen werden, da entsprechende Rentenleistungen betragsmäßig wegen ihrer Höhe und zeitlichen Befristung relativ unbedeutend sind, außerdem von einer Fürsorgepflicht der Gesellschaft, unter Umständen auch der Versichertengemeinschaft, ausgegangen werden kann, die weit über das bei Ehegatten angemessene Maß hinausgeht.

96 Somit werden typische Fälle von Moral hazard ermöglicht. Für Ledige, die auch in Zukunft ledig bleiben wollen, läßt sich ein Teil des Beitrags als Sondersteuer ("Junggesellensteuer") interpretieren. Anderer Auffassung ist Kolb (1985, 130f.), allerdings ohne wirklich schlüssige Argumente vorzulegen.

97 Eine solche Behandlung ist angezeigt, weil sonst der Beitragsteil zur Finanzierung der Hinterbliebenensicherung, der sich als Sondersteuer auf das Einkommen von Ledigen interpretieren läßt, nochmals der Einkommensteuer unterworfen würde. 
Todesfalleistung an den Bezugsberechtigten - bedingt um eine Versicherung zugunsten Dritter. Eine Aufspaltung des Beitrags nach den beiden Versicherungsarten ist dann notwendig. Anteilige Beiträge an eine Todesfallversicherung sind aus versteuertem Einkommen zu leisten. Da der Versicherungsnehmer im Regelfall alle (aktuellen) Rechte aus der Versicherung einschließlich der (Um-)Benennung eines Bezugsberechtigten besitzt, lassen sich Leistungen an den Bezugsberechtigten erst zum Zeitpunkt des effektiven Zuflusses bei diesem als Schenkung einkommensteuerlich erfassen. Lediglich bei unwiderruflicher Festlegung eines einzigen Bezugsberechtigten sowohl für den Erlebens- als auch für den Todesfall gehen alle Vermögensrechte auf diesen über, so daß dann bereits die Beiträge als Transfer zu versteuern sind, die Wertentwicklung der Versicherung beim Bezugsberechtigten als Vermögenszugang zu erfassen ist. Dem Versicherungsnehmer bleiben neben den Versicherungspflichten lediglich die Rechte der vorzeitigen Kündigung oder Umwandlung in eine beitragsfreie Versicherung, wovon aber die in der Vergangenheit abgetretenen Leistungen nicht mehr berührt werden 98 .

Von besonderem Interesse sind Todesfallrisikoversicherungen, die ein Todesfallrisiko lediglich für einen bestimmten Zeitraum abdecken und sich damit besser als andere Versicherungsformen auf bestimmte Bedarfssituationen abstimmen lassen (Reuter, 1987, 99). Diese Versicherungen gehen in vollem Umfang zugunsten Dritter, sind daher - bei Fehlen besonderer Umstände - in vollem Umfang aus versteuertem Einkommen zu bestreiten; bei Eintritt des Versicherungsfalles sind die Leistungen beim Begünstigten ebenfalls als steuerpflichtige Einkünfte zu erfassen.

$98 \mathrm{Zu}$ den möglichen Vertragsgestaltungen in bezug auf die Personen des Versicherungsvertrages vgl. Hagelschuer (1983, 61-65). Für den bisher nicht behandelten Fall, daß die Rechte aus dem Erlebens- und Todesfall unwiderruflich an zwei unterschiedliche Personen vergeben werden, könnte für einkommensteuerliche Zwecke bis zum Eintritt des Versicherungsfalls der Verbleib aller Rechte beim Versicherungsnehmer als Fiktion dienen. 


\section{Sicherung im Krankheitsfall}

Während im Rahmen der Alterssicherung die Absicherung gegen zukünftige Risiken im Vordergrund stand, handelt es sich bei der Krankenversicherung vor allem um ein Instrument zur Absicherung gegen laufende Risiken. Die Behandlung der Lohnersatzzahlungen einer Krankenversicherung steht damit stellvertretend auch für andere Sicherungsinstitutionen, bei denen laufende Risiken des Einkommensausfalls abgedeckt werden, wie das bei der Arbeitslosenversicherung und der Unfallversicherung der Fall ist.

\subsection{Charakterisierung}

Die Leistungen von Krankenversicherungen lassen sich in zwei große Gruppen einteilen: einmal die monetären Leistungen zum Ausgleich eines krankheitsbedingten Einkommensausfalls, die bei Arbeitnehmern i.d.R. einsetzen, sobald die Lohnfortzahlung im Krankheitsfall durch den Arbeitgeber wegfällt, zum anderen die Kostenerstattung für in Anspruch genommene Sachleistungen, die entweder an den Versicherten oder direkt an den Leistungserbringer erfolgen kann 99.

Während die Träger der gesetzlichen Krankenversicherung Leistungen nach einem weitgehend festgelegten Katalog erbringen und ihre Beiträge nach sozialen Gesichtspunkten differenzieren, so insbesondere nach der Höhe des Arbeitseinkommens, und gleichzeitig Familienmitglieder ohne eigenes Einkommen beitragsfrei mitversichern, bemühen sich die Träger der privaten Krankenversicherung um eine nach dem individuellen Risiko bei Eintritt in die Versicherung gestaffelte Beitragsdifferenzierung bei gleichzeitigen umfassenden Wahlmöglichkeiten unter verschiedenen Leistungsangeboten. Für Arbeitnehmer (ausgenommen Beamte) besteht i.d.R. eine Pflichtmitgliedschaft in der gesetzlichen Krankenversicherung bis zur Versicherungspflichtgrenze (1993: 5400

99 Auch die direkte Kostenübernahme gegenüber dem Leistungserbringer läßt sich als monetäre Leistung auffassen, da hier keine Probleme der Zurechnung und Bewertung (wie sonst bei realen Transfers) auftreten. Vgl. Transfer-Enquête-Kommission $(1979,41$ f.) und Lukarsch $(1988,960)$. 
DM monatlich). Für alle nicht pflichtversicherten Personengruppen besteht Versicherungsfreiheit, was unter bestimmten Umständen die Wahlmöglichkeit zwischen den Angeboten der gesetzlichen und privaten Krankenversicherung beinhaltet (vgl. hierzu §§ 5-10 SGB V). 99,6\% der deutschen Bevölkerung sind krankenversichert, davon $89,3 \%$ bei einem zur gesetzlichen Krankenversicherung zu rechnenden Träger versichert oder mitversichert, $8,4 \%$ bei einem Unternehmen aus dem Bereich der privaten Krankenversicherung, 2\% verfügen über einen sonstigen Versicherungsschutz (freie Heilfürsorge). Lediglich 0,4\% der Bevölkerung verfügen über keine Krankenversicherung (Sommer, 1987, 253).

\subsection{Steuersystematische Aspekte}

\subsubsection{Sicherung gegen krankheitsbedingten Einkommensausfall}

(1) Werbungskostencharakter der Aufwendungen

Bei der Versicherung des laufenden Arbeitseinkommens für den Fall der Krankheit handelt es sich dem Gesetzeswortlaut des $\S 9$ EStG folgend ganz klar um Werbungskosten ("Aufwendungen zur Erwerbung, Sicherung und Erhaltung der Einnahmen"). Sie sind entsprechend bei den Einkünften in Abzug zu bringen, zu denen auch das Krankengeld gerechnet wird. Daß sie derzeit nicht als Werbungskosten, sondern als Sonderausgaben (spezieller: Vorsorgeaufwendungen) behandelt werden, liegt auch an der Steuerfreiheit der damit erzielten Einnahmen 100. Nach dem hier vertretenen Konzept sollten die Einkommensersatzleistungen der Krankenversicherung ohnehin zu den steuerpflichtigen Einkünften gerechnet werden 101 .

Die derzeitige Regelung sieht im Rahmen der gesetzlichen Krankenversicherung eine Festlegung der Lohnersatzleistungen auf $80 \%$ des regelmäßigen (Brutto-)Arbeitsentgelts, maximal jedoch auf die Höhe des

100 Ein Werbungskostenabzug von Aufwendungen, die mit steuerfreien Einnahmen in Zusammenhang stehen, ist nach $\S \S 8$ und 9 EStG ausgeschlossen.

101 Vgl. Kapitel III.1. 
regelmäßigen Nettoarbeitsentgelts, vor ( $\S 47$ SGB V) ${ }^{102}$. Damit verbunden ist eine Steuerfreiheit der Leistungen ( $\$ 3 \mathrm{Nr} .1 \mathrm{a} \mathrm{EStG}$ ), die jedoch dem Progressionsvorbehalt nach $\S 32 \mathrm{~b}$ EStG unterliegen, und eine durch die Höchstbeträge für Vorsorgeaufwendungen begrenzte Abzugsfähigkeit der Beiträge. Unter dem Aspekt der Steuergerechtigkeit können diese Regelungen nicht überzeugen.

\section{(2) Weitere Aspekte}

Mit einer Zuordnung der Krankenversicherungsbeiträge zu den Werbungskosten und der steuerlichen Erfassung des Krankengeldes wird gleichzeitig auch die Steuerfreiheit des Existenzminimums im Krankheitsfall gewährleistet, da das Krankengeld bis zur Höhe des Existenzminimum-Grundfreibetrages steuerfrei bleibt, während nach der derzeitigen Rechtslage zwar nicht das Krankengeld besteuert wird, aber die dafür aufgewendeten Versicherungsbeiträge zum Teil aus versteuertem Einkommen geleistet werden.

Auch dient es einer Erhöhung der intertemporalen Gleichmäßigkeit der Besteuerung, wenn Aufwendungen zur Glättung von Einkommensschwankungen einen Abzug von der Steuerbemessungsgrundlage erfahren und die damit erzielten Lohnersatzeinkommen einkommensteuerlich erfaßt werden.

Durch den Werbungskostenabzug der Beiträge wird weiterhin dem jedenfalls für Arbeitnehmer i.d.R. bestehenden - Versicherungszwang und der damit einhergehenden Reduktion des frei verfügbaren Einkommens Rechnung getragen. Meritorische Aspekte lassen sich insbesondere für eine Grundsicherung durch Lohnersatzeinkommen anführen, bleiben jedoch auch hier schwer in eine Förderkonzeption umsetzbar. Stattdessen bietet sich ein Versicherungszwang an.

102 Auch die private Krankenversicherung orientiert sich an der Absicherung des Nettoeinkommens. 


\subsection{Absicherung von krankheitsbedingten Kostenbelastungen}

\section{(1) Sicherung des Existenzminimums}

Maßnahmen zur Erhaltung und Wiederherstellung der Gesundheit werden allgemein als existentiell notwendig eingestuft. Daher sind Leistungen im Rahmen einer Kostenerstattung oder direkt als Sachleistungen -, die diesem Zweck dienen und einen als notwendig erachteten Umfang nicht überschreiten, dem steuerlichen Existenzminimum zuzurechnen, das wegen der besonderen Belastungen durch den Krankheitsfall entsprechend höher anzusetzen ist (Lang, 1988, 206-209). Solche Leistungen erhöhen bei Einbezug der Ausgabenseite die steuerliche Leistungsfähigkeit nicht, sie dienen lediglich dazu, krankheitsbedingte Leistungsfähigkeitsminderungen von Steuerpflichtigen aufzufangen (Andrews, 1972/73, 314)103. Wenn aber die Behandlungskosten im Krankheitsfall als Teil des Existenzminimums einzustufen sind, dann sollte dies gleichermaßen für Versicherungsbeiträge gelten, die zu einer Absicherung dieser Kostenbelastung führen. Daß eine Zuordnung der krankheitsbedingten Leistungen zum Existenzminimum richtig ist, zeigt auch ein Blick auf entsprechende Sozialhilfeleistungen ( $\S 13,37$ BSHG) 104 .

Der Umfang der Absicherung sollte allerdings das gesellschaftlich als notwendig erachtete Maß nicht übersteigen. Die Leistungen der gesetzlichen Krankenversicherung geben die Vorstellungen über den als notwendig erachteten Umfang recht gut wieder (vgl. auch § 13 BSHG). Probleme wirft allerdings der Umstand auf, daß die Beitragsbelastung in der gesetzlichen Krankenversicherung von der Erwerbstätigkeit (Familienmitversicherung!) und der Höhe des Arbeitseinkommens der Steuerpflichtigen abhängt (was lediglich beim Krankengeld seine Entsprechung auf der Leistungsseite findet), so daß dem Existenzminimum kein fixer Betrag für eine Absicherung im Krankheitsfall zugerechnet werden

103 Auch hier wird rein materiell argumentiert, andere Faktoren müssen außer Betracht bleiben.

104 Trotzdem lehnen Pauly (1986) und Phelps (1984/85) die Steuerfreiheit von Krankenversicherungsbeiträgen als unangemessene Steuervergünstigung ab. 
kann. Auch gibt es bestimmte Bereiche, in denen ein Bedarf zwar anerkannt wird, eine volle Kostenübernahme durch die Träger der gesetzlichen Krankenversicherung aber nur für bestimmte als besonders bedürftig eingeschätzte Personengruppen vorgesehen ist ( $\S 61$ und 62 SGB V). Die Steuerfreiheit von Selbstbeteiligungen der Versicherten in diesen Bereichen ist daher gesondert sicherzustellen, da sie zwar dem Existenzminimum zuzurechnen sind, jedoch über die Einkommensteuerbefreiung der entsprechenden Krankenversicherungsbeiträge und -leistungen nicht voll berücksichtigt werden.

\section{(2) Konsumelemente einer Krankenversicherung}

Während Aufwendungen zur Absicherung des Existenzminimums von der Einkommensteuer freizustellen sind und damit eine Ausnahme vom Grundsatz der steuerlichen Unerheblichkeit der Einkommensverwendung darstellen, sind darüber hinausgehende Aufwendungen, auch wenn sie der Erhaltung oder Wiederherstellung der Gesundheit des Steuerpflichtigen dienlich sind, aus versteuertem Einkommen zu leisten. Sofern der hier vorgetragenen Argumentation gefolgt wird, wären das alle Leistungen, die über den Leistungskatalog der gesetzlichen Krankenversicherung hinausgehen. Eine solche Einschränkung der steuerlichen Berücksichtigung scheint deshalb sinnvoll, weil mit dem Leistungsstandard der gesetzlichen Krankenversicherung ein für den Großteil der Bevölkerung geltender Rahmen gefunden ist, der als sozio-kulturelles gesundheitliches Existenzminimum angesehen werden kann. Diesen Rahmen übersteigende Ausgaben können zwar durchaus ebenfalls krankheitsbedingt sein, stellen aber Ausgaben der privaten Lebensführung dar, was sich aus dem Umstand ergibt, daß sie aus Sicht der gesetzlichen Krankenversicherung - und ein anderer gesellschaftlicher Konsens zu der Frage ist mir nicht bekannt - objektiv nicht notwendig zur Erhaltung oder Wiederherstellung der Gesundheit sind. Der Vergleich mit gesunden Steuerpflichtigen gleichen Lebensstandards drängt sich zwar auf, und einiges spricht dafür, daß mit steigendem Lebensstandard auch die krankheitsbedingten Aufwendungen ein "Existenzminimum" übersteigen, jedoch sind diese Ausgaben wiederum der individuellen Lebensgestaltung zuzurechnen, da sie einen 
besonderen Luxus von Kranken widerspiegeln (z.B. Chefarztbehandlung, Einbettzimmer, aufwendige Kuren), auch wenn die Grenzziehung eine Frage des Werturteils bleibt. Die Durchbrechung des ExistenzminimumKonzepts in dieser Frage (Lang, 1988, 595f.) ist nicht konsequent. Allerdings liegen zum Teil auch Abweichungen vom Konsens vor, die nicht den betriebenen Aufwand betreffen, sondern die Art der Behandlung, wie z.B. durch Heilpraktiker, was problematischer ist.

\section{(3) Meritorische Aspekte}

Durch Vorsorge in Form einer Krankenversicherung wird der existentielle Bedarf im Krankheitsfall abgedeckt. Dadurch ist gewährleistet, daß dem Staat keine zusätzlichen Ausgaben in Form von Sozialhilfe entstehen. Hinzu kommt die Überlegung, daß durch eine wirksame Krankheitsvorsorge und -behandlung andere Mitglieder der Gesellschaft vor möglichen Krankheitsübertragungen geschützt werden, ein klassischer Fall positiver externer Effekte. Damit gibt es gute Gründe für staatliche Eingriffe. Ob allerdings zu einer einkommensteuerlichen Freistellung von Beiträgen und Leistungen noch eine Förderprämie hinzukommen sollte (insb. wegen des zweiten Arguments, da als Reaktion auf das erste eine Versicherungspflicht angemessen ist), bleibt insgesamt fraglich.

\section{(4) Zwang}

Durch den Versicherungszwang sind die gegenwärtigen Möglichkeiten zur Steuerzahlung durch die Abführung von Zwangsbeiträgen eingeschränkt. Sofern den Zwangsbeiträgen entsprechende Gegenleistungen gegenüberstehen, vermag dieses Argument für sich genommen allerdings nicht zu überzeugen. Im Fall der gesetzlichen Krankenversicherung ist allerdings der Anteil von Leistungen der interpersonellen Umverteilung besonders hoch, so daß sich wegen des steuerähnlichen Charakters eines Teiles der Krankenversicherungsbeiträge ein weiteres Argument für eine (partielle) Steuerbefreiung ergibt 105 .

105 Vgl. Kapitel III.3.2. 


\section{(5) Intertemporaler Ausgleich}

Fragen des intertemporalen Belastungsausgleichs scheinen auf den ersten Blick in der Krankenversicherung, in der es um den laufenden interpersonalen Belastungsausgleich geht, keine Rolle zu spielen. Wegen des mit zunehmendem Alter steigenden Krankheitsrisikos läßt sich bei vom Lebensalter unabhängigen Beiträgen jedoch durchaus eine solche intertemporale Komponente feststellen. Der entsprechende Beitragsanteil ist als eine Form der Alterssicherung zu betrachten. Da es große Ähnlichkeiten mit den Modalitäten der Leibrentenversicherung gibt (lebenszeitbezogene Leistungen nach dem aktuellen "Bedarf", der allerdings innerhalb der Leibrentenversicherung fix, innerhalb der Krankenversicherung variabel ist), sollte idealerweise auch die einkommensteuerliche Behandlung angeglichen werden.

\subsection{Umsetzung in die Steuerpraxis}

Sofern Beiträge zur Sicherung des krankheitsbedingten Einkommensausfalls als Werbungskosten berücksichtigt werden, sind damit gleichzeitig die Forderungen nach steuerlicher Berücksichtigung einer Sicherung des Existenzminimums und der intertemporalen Ausgleichsfunktion erfüllt. Ob zusätzlich eine Förderung - jedenfalls von Beiträgen, die eine Grundsicherung bewirken - vorzusehen ist, läßt sich unter Leistungsfähigkeitsaspekten allein nicht entscheiden, sondern hängt vor allem auch vom Gesamtkonzept staatlicher Förderprämien ab.

Da Beiträge zur gesetzlichen Krankenversicherung, sofern sie der Absicherung gegen krankheitsbedingte Belastungen dienen, lediglich eine Sicherung des Existenzminimums bewirken, sind sie von der Einkommensteuer freizustellen. $\mathrm{Da}$ es sich hier nicht um Werbungskosten handelt ${ }^{106}$, kommt formal nur eine Berücksichtigung im Grundfreibetrag oder ein Abzug von der Summe der Einkünfte in Frage. Eine pauschale Berücksichtigung im Grundfreibetrag scheidet allerdings ebenfalls aus, da

106 Es sei denn in dem weiten Zusammenhang, daß die Gesundheit zur Erzielung von Arbeitseinkommen notwendig ist, dann aber "lebt der Mensch, um zu arbeiten" (vgl. Simons, 1938, 74). 
die Beiträge zur gesetzlichen Krankenversicherung mit der Höhe des Arbeitseinkommens variieren; also bietet sich vor allem die Möglichkeit eines Abzugs von der Summe der Einkünfte an ${ }^{107}$. Auch dem gedanklichen Konstrukt einer "Rücklage für das Alter" wird mit einer solchen Behandlung entsprochen: Die Steuerfreiheit von Beiträgen und Leistungen wird gewahrt, auch eine Aufspaltung des Beitrags gemäß den Zwecken "laufende Absicherung gegen Krankheitskosten" und "Zukunftsvorsorge" würde am Ergebnis der steuerlichen Behandlung nichts ändern. Dem "Zwangsargument" wird durch einen Abzug von der Summe der Einkünfte ebenfalls Rechnung getragen. Es bleibt lediglich die Frage einer zusätzlichen Förderung von Krankenversicherungen, deren Beantwortung auch hier wieder vom Gesamtkonzept von Fördermaßnahmen abhängt, jedenfalls bei einer insgesamt restriktiven Handhabung aber verzichtbar erscheint.

\subsection{Zum Verhältnis von Risikoselbsttragung und Versicherung}

Im Fall der Versicherung gegen den Ausfall von Arbeitseinkommen fällt ein Urteil leicht. Wer das Instrument der Versicherıng nicht in Anspruch nimmt, erreicht auch nicht den damit möglichen intertemporalen Ausgleich 108. Die steuerliche Bemessungsgrundlage wird korrekt erfaßt, allerdings kann durch die Tarifprogression eine vergleichsweise stärkere steuerliche Belastung eintreten, was dann aber ein allgemeines Problem der Einkommensteuer darstellt ${ }^{109}$. Zu erwägen ist jedoch auch hier eine (pauschale) steuerliche Entlastung von Vermögensauflösungen, die ledig-

107 Die Berücksichtigung innerhalb einer Einkunftsart als Werbungskosten, z.B. im Rahmen der "Sonstigen Einkünfte", würde eine Steuerpflicht der Sachleistungen von Krankenversicherungen voraussetzen, die konzeptionell bei gleichzeitigem Ansatz von besonderen Belastungen im Rahmen des Existenzminimums zwar möglich ist, jedoch zu unnötigen Komplizierungen der Steuerveranlagung führen würde.

108 Insofern ist dieser Fall mit dem Einsatz der allgemeinen Vermögensbildung zur Alterssicherung vergleichbar.

109 Eine entsprechende allgemeine Lösung stellt die Verwendung geeigneter Methoden der interperiodischen Durchschnittsbesteuerung bzw. Lebenseinkommensbesteuerung dar. 
lich das laufende Existenzminimum von Steuerpflichtigen gewährleisten 110 .

Im Fall der krankheitsbedingten Belastungen ist aus Gleichbehandlungsgründen bei Selbsttragung des Risikos eine einkommensteuerliche Berücksichtigung der Aufwendungen durch einen Abzug von der Bemessungsgrundlage zu gewährleisten, sofern ihre Höhe vergleichbare Leistungen der gesetzlichen Krankenversicherung nicht übersteigt. Der Ansatz einer zumutbaren Belastung ( $\$ 33 \mathrm{EStG}$ ) ist dabei nicht angebracht. Allerdings kann durch eine Selbsttragung von Risiken i.d.R. nicht sichergestellt werden, daß die Allgemeinheit von einer Übernahme von Kosten verschont bleibt, wie das bei einer Krankenversicherung der Fall ist. Aus diesem Grunde läßt sich eine Besserstellung der Krankenversicherung oder aber eine Versicherungspflicht für alle rechtfertigen.

\subsection{Besonderheiten der privaten Krankenversicherung}

Private Krankenversicherungen sind bezüglich der Beiträge und Leistungen prinzipiell gleich wie gesetzliche Krankenversicherungen $\mathrm{zu}$ behandeln. Allerdings sind Beiträge zu einer Krankheitskostenversicherung, die höhere Leistungen bewirken, als sie in der gesetzlichen Krankenversicherung üblich sind, getrennt auszuweisen und von einer einkommensteuerlichen Berücksichtigung auszuschließen. Grundlage hierfür ist das oben dargelegte Konzept einer Gleichsetzung von "gesetzlichen" Leistungen und Existenzminimum. Die diesbezüglichen Berechnungen müßten vereinfachungshalber aufgrund der Leistungsanteile an einen geschlossenen Versichertenbestand erfolgen, sie könnten weder individuelle Verhältnisse noch Beitragsrücklagen berücksichtigen. Bei Versicherungen gegen krankheitsbedingten Einkommensausfall sind keine besonderen von den Verhältnissen der gesetzlichen Krankenversicherung abweichenden Bedingungen $\mathrm{zu}$ beachten; eine Überversicherung ist ausgeschlossen 111 , zudem dürfte aufgrund der Progressionseffekte sowohl eine Über-

110 Vgl. Kapitel V.1.2.1.

111 Vgl. § 4(2) der Musterbedingungen 1978 des Verbandes der privaten Krankenversicherung - MB/KT 78 - (Aumüller/Balzer, 1991, XXXII). 
als auch eine Unterversicherung zu Lasten der Steuerpflichtigen gehen. $\mathrm{Ob}$ die sich so ergebenden Beitragskomponenten größer oder kleiner als die der gesetzlichen Krankenversicherung sind, ist dann steuerlich unerheblich: sie sollten jedenfalls in ihrer vollen Höhe als Werbungskosten bzw. von der Summe der Einkünfte abzugsfähig sein.

Ein wesentlicher Unterschied zwischen gesetzlicher Krankenversicherung und privater Krankenversicherung liegt in den (Ex-ante-) Umverteilungskomponenten der gesetzlichen Krankenversicherung. Eine einkommensteuerliche Berücksichtigung der daraus resultierenden Beitragsdifferenzen, die über den vorgeschlagenen Abzug von der Steuerbemessungsgrundlage hinausgeht, ist jedoch in konzeptionell sinnvoller Weise nicht möglich. Eine gleichmäßige(re) Beteiligung aller leistungsfähigen Bürger an der Finanzierung der angesprochenen Umverteilungskomponenten ist nur durch eine Ausweitung der Versicherungspflicht in der gesetzlichen Krankenversicherung oder durch eine Steuerfinanzierung der nicht beitragsäquivalenten Leistungen der gesetzlichen Krankenversicherung möglich. 
Harald Schlee - 978-3-631-75216-6

Downloaded from PubFactory at 01/11/2019 06:59:36AM

via free access 


\section{TRANSFERS ZWISChEN PRIVATEN, INSBESONDERE INNERFAMILIÄRE TRANSFERS}

\section{Zur Bedeutung der Familie für die individuelle soziale Sicherung}

Eine klassische Funktion der Familie besteht aus ökonomischer Sicht in der Bereitstellung innerfamiliärer Transfers zur Verwirklichung sozialer Sicherheit. Zu nennen ist hier vor allem die Versorgung von Kindern und Alten sowie - innerhalb der gleichen Generation - zwischen Ehepartnern. Die Familie als soziales Sicherungssystem kann in Analogie zu Versicherungen als Gefahrengemeinschaft betrachtet werden, deren konstituierendes Element ein ausgewogenes Verhältnis von Rechten und Pflichten darstellt. Die im Bürgerlichen Gesetzbuch formulierten gegenseitigen Unterhaltsverpflichtungen von "Verwandten in gerader Linie" scheinen in Verbindung mit dem Erbrecht diesen Bedingungen zu genügen. Bei näherer Betrachtung der tatsächlichen Gegebenheiten zeigt sich jedoch, daß die Familie seit der Ablösung der traditionellen Großfamilie im Gefolge der Industrialisierung als in sich autarkes soziales Sicherungssystem durch Öffnung nach außen insgesamt stark an Bedeutung verloren hat. Hinzu kommt, daß sie in ein inneres Ungleichgewicht bezüglich der Verteilung von Rechten und Pflichten zwischen den Generationen geraten ist ${ }^{1}$.

In der traditionellen Großfamilie war es so, daß die jeweils erwerbstätige Generation den Unterhalt von Alten und Kindern sicherte, gleichzeitig auch selbst durch Einbezug in diesen Unterhaltsverbund Aussicht auf zukünftige Leistungen hatte. Allerdings wurde die erwerbstätige Generation durch Mithilfe der nicht voll erwerbstätigen Familienangehörigen und die Übereignung vorhandenen Vermögens, das i.d.R. als "Betriebskapital" diente, unterstützt. Es bleibt jedoch auch festzuhalten, daß die Unterhaltsgewährung ein recht bescheidenes Ausmaß hatte, wegen der jeweiligen individuellen Situation erhebliche

1 Vgl. zu diesem Bereich insgesamt Ruland (1973, 28f., 235-237) sowie Albers (1967b, 160f.). 
Sicherungslücken und Belastungsunterschiede auftraten und die persönlichen Beziehungen aufgrund der Befrachtung mit materiellen Verpflichtungen großen Belastungen ausgesetzt waren².

Mit dem Rückgang der Bedeutung der Familie als Produktionsgemeinschaft und der Zunahme außerhäuslicher Erwerbstätigkeit wurde die Kleinfamilie, bestehend aus Eltern und ggf. unterhaltsberechtigten Kindern, zum Regelfall. Gleichzeitig wurden die Sicherungsfunktionen der Großfamilie in Teilen durch die verschiedenen Zweige der Sozialversicherung abgelöst. Den Anknüpfungspunkt für diese familienunabhängige Sicherung bot die außerhäusliche Erwerbstätigkeit. Die Sicherung wurde jedoch nicht auf die Erwerbstätigen selbst beschränkt, sondern in Analogie zu deren Unterhaltsverpflichtungen innerhalb der "Kleinfamilie" durch ein System abgeleiteter Sicherung für Familienangehörige ergänzt (z.B. Familienmitversicherung in der gesetzlichen Krankenversicherung, Hinterbliebenensicherung in der gesetzlichen Rentenversicherung). Schließlich ist der Ausbau der Sozialhilfe als subsidiäre Sicherung zu nennen.

Besonders deutliche Veränderungen vollzogen sich dadurch in der Sicherung alter Menschen, die durch die gesetzliche Rentenversicherung und andere Formen der Alterssicherung materiell weitgehend von persönlichen Unterhaltsansprüchen unabhängig wurden ("sozialisierte" Altersversorgung). Soweit zur Zeit noch Sicherungslücken bestehen, wird an deren Schließung weiter gearbeitet (z.B. Pflegeversicherung, Anerkennung von Kindererziehungszeiten). Der gestiegenen Unabhängigkeit der alten Menschen entspricht eine Entlastung der Kinder von persönlichen Unterhaltsverpflichtungen, womit die Symmetrie von Rechten und Pflichten in der intergenerationalen Solidargemeinschaft "Familie" gestört ist. Allerdings steht dieser Entlastung der Kindergeneration eine Belastung durch Beiträge für die eigene Altersvorsorge gegenüber. $\mathrm{Zu}$ ergänzen ist diese Betrachtung um die Ansprüche auf Vermögensübertragung durch Erbschaft, denen erst recht dann keine Unterhalts-

2 So versucht Knorr-Anders (1988) unter dem Titel "Am besten wär's, euch zeitig totzuschlagen" die nostalgische Sicht der Großfamilie zurechtzurücken. 
verpflichtungen gegenüberstehen, wenn nach der konkreten Vermögenssituation potentiell Unterhaltsberechtigter alter Menschen differenziert wird ${ }^{3}$.

Auch heute noch stark ausgeprägt ist der Unterhaltsverband zwischen Eltern und unterhaltsberechtigten Kindern. Hier greifen zwar auch sozialrechtliche Regelungen wie das Bundeskindergeldgesetz und das Bundesausbildungsförderungsgesetz unterstützend ein, der größere Teil der materiellen Belastung verbleibt aber bei den Eltern; hinzu kommt die Belastung durch persönliche Betreuung. Aufgrund der "Vergesellschaftung der Altersversorgung" steht den Leistungen der Eltern zugunsten von Kindern nicht mehr die Erwartung einer späteren Gegenleistung gegenüber, worin eine entscheidende Ursache für Fehlentwicklungen im generativen Verhalten gesehen wird (Albers, 1989, 342f.).

Zwischen Ehepartnern hängt die faktische Bedeutung von Unterhaltsbeziehungen sowohl in der "Aktivenphase" als auch im Alter entscheidend von deren Beteiligung am Erwerbsleben ab; ist (war) nur einer der Ehepartner erwerbstätig, dann kommen die rechtlichen Unterhaltsbestimmungen voll zum Tragen, sind (waren) beide Ehepartner erwerbstätig, was zunehmend der Fall ist ${ }^{4}$, dann werden die Unterhaltsverpflichtungen relativ bedeutungslos. $\mathrm{Zu}$ berücksichtigen ist auch, daß in steigendem Ausmaß die Ehe selbst durch andere Lebensformen abgelöst wird 5 , in

3 Zur konstitutionellen Verknüpfung von Erbrecht und Unterhaltsrecht vgl. Ruland (1973, 223f.) und Brox (1991, 11).

4 So kamen im Jahr 1989 die altersspezifischen Erwerbsquoten (25-50 Jahre) bei Männern nahe an $100 \%$, bei verheirateten Frauen immerhin nahe an $60 \%$ (Statistisches Bundesamt, 1991, 113). Das ist deshalb besonders bemerkenswert, weil Zeiten der Kinderbetreuung in die genannte Lebensphase fallen.

$5 \mathrm{Zu}$ nennen sind hier Haushalte von Alleinlebenden, von nichtehelichen Lebensgemeinschaften und von Alleinerziehenden, die offenbar auch bewußt als Lebensform gewählt werden (vgl. Bundesminister für Jugend, Familie, Frauen und Gesundheit, 1986, 36-38). 
denen eine vergleichbare unterhaltsrechtliche Sicherung nicht mehr besteht (Geschiedene) oder ohnehin nicht besteht (Ledige) 6 .

Abschließend läßt sich feststellen, daß das traditionelle Familienmodell in verschiedener Hinsicht nicht mehr zeitgemäß ist: Zum einen wurde die Belastung der familiären Beziehungen mit materiellen Ansprüchen reduziert, wie sowohl der Aufbau einer familienunabhängigen Sicherung gegen Lebensrisiken als auch die Einschränkung von Unterhaltsverpflichtungen im Rahmen der Sozialhilfe-Regreßansprüche (Brühl, 1989, 225-228) zeigt, so daß die persönliche Beziehung der Familienmitglieder stärker in den Mittelpunkt rücken konnte (D.Schwab, 1991, 2f.). Zum anderen ist die Instabilität der Ehe als "Kern" der traditionellen Familie von der Intensivierung anderer inner- und außerfamiliärer Beziehungen begleitet (Segalen, 1990, 203f.), die zum großen Teil zivil-, sozial- und steuerrechtlich nicht typisiert sind.

\section{Haushalts- oder Individualbesteuerung?}

Von grundlegender Bedeutung für den weiteren Verlauf der Diskussion ist die Frage nach dem Träger steuerlicher Leistungsfähigkeit, nach der Subjekteinheit der Besteuerung. Dabei geht es im Kern nicht darum, ob die Lebensumstände von Individuen (hier: allein lebend oder gemeinsam mit anderen Personen in einem Haushalt) steuerlich berücksichtigt werden sollten, worauf sich die Differenzierung zwischen Individual- und Haushaltsbesteuerung in der Literatur jedoch i.d.R. bezieht 7 . Vielmehr geht es um die Bestimmung des Adressaten steuerlicher Maßnahmen (wie z.B.

6 So ist damit zu rechnen, daß 30-35\% der heute geschlossenen Ehen wieder geschieden werden (Paul, 1990, 838f.), auch hat die Heiratsneigung, gemessen an der Zahl der Unverheirateten im heiratsüblichen Alter, erheblich abgenommen (Bundesminister für Jugend, Familie, Frauen und Gesundheit, 1986, 32-35). Vgl. hierzu auch Segalen (1990, 201-203), die insbesondere auch die Entwicklung im Ausland schildert.

7 Deshalb kommt es hier zu einer Begriffsverwirrung. Charakteristisch für die üblicherweise in der Literatur anzutreffende Zuordnung ist die Kennzeichnung der Individualbesteuerung als "berufsbezogen", der Haushaltsbesteuerung dagegen als "familienbezogen" (Jecht, 1955/56, 45-47; Kullmer, 1960, 53-57). 
der Tarifprogression); dabei können sowohl bei einer individual- als auch bei einer haushaltsbezogenen Sichtweise persönliche Lebensumstände berücksichtigt werden.

In der Literatur finden sich nur wenige Autoren, die versuchen, diese Frage anzugehen (Hackmann, 1979, 39-43; v. Obstfelder, 1976, 164-169; Royal Commission on Taxation, 1966, Bd.3, 122-141). In der Regel wird der Kern der - z.T. bereits explizit präzisierten (Albers, 1980, 194f.) Fragestellung umgangen.

Für das Individuum als Subjekteinheit der Einkommensteuer spricht zunächst, daß unser gesamtes Gesellschaftssystem - soweit möglich - auf das Individuum als grundlegende Einheit bezogen ist, wie es in der Formulierung individueller Rechte im Grundgesetz deutlich wird. Hierauf dürfte auch die Betonung der Individualbesteuerung durch das Bundesverfassungsgericht $(1957,67 ; 1959,243)$ beruhen. Auch eine nutzenorientierte Betrachtungsweise wird zwangsläufig beim Individuum ansetzen (Hackmann, 1979, 40f.).

Es könnte allerdings eine Frage der Zweckmäßigkeit sein, zu Haushalten zusammengeschlossene Individuen als gesonderte Besteuerungseinheit "Haushalt" zu behandeln, um den Besonderheiten des Zusammenlebens in einem gemeinsamen Haushalt in ihrer Summe Rechnung zu tragen und ein Eindringen in die Privatsphäre für die Feststellung haushaltsinterner Transfers zu vermeiden.

Zum einen spricht gegen eine solche Vorgehensweise die Verschiedenartigkeit von Haushalten hinsichtlich der rechtlichen und ökonomischen Beziehungen der Haushaltsangehörigen zueinander. Eine pauschalierende Betrachtung der Einheit "Haushalt" würde zahlreiche vorhandene Informationen über die jeweilige individuelle Situation der Haushaltsangehörigen (z.B. die Charakterisierung als Erwerbsgemeinschaft, als Unterhaltsgemeinschaft oder als Zusammenleben rechtlich ungebundener Personen) unberücksichtigt lassen oder aber durch eine sehr restriktive Definition eines Modellhaushalts alle anderen Arten gemeinsamer Haushaltsführung aus der Betrachtung ausschließen. Dagegen erlaubt eine Besteuerung von Individuen neben der Würdigung der jeweiligen individuellen Situation auch die Berücksichtigung rechtlicher Unterhalts- 
verpflichtungen und steuerlich relevanter Gesichtspunkte des Zusammenlebens. Dazu genügt bereits der Rückgriff auf Pauschalierungen unter Verwertung allgemein bekannter Informationen, so daß ein Eindringen in die Privatsphäre vermieden werden kann.

Zum anderen ist dem Sachverhalt Rechnung zu tragen, daß es sich bei Haushalten um im Zeitablauf instabile Einheiten handelt, deren Mitglieder vor einem Eintritt in bzw. nach einem Austritt aus einem gemeinsamen Haushalt als Individuen zu besteuern sind. Auch bei einer Besteuerung von Haushalten, die grundsätzlich auf die Erfassung haushaltsinterner Transfers verzichten könnte, sind daher Annahmen über die individuelle Zuordnung steuerlicher Leistungsfähigkeit zu treffen, die sich explizit in der steuerlichen Behandlung von Transfers bei Ein- und Austritt aus einem gemeinsamen Haushalt zeigen. Auch ist für den Fall der Aufspaltung von Haushalten in Individuen sicherzustellen, daß Regelungen für eine Besteuerung von Transfers innerhalb von Haushalten mit denjenigen für Individuen konsistent sind. Offensichtlich wird auch dieser Bedingung am besten eine durchgängig individualorientierte Betrachtung gerecht, die auch für die Besteuerung von Haushalten explizit die Bezugsbasis bildet ${ }^{8}$.

Da zumindest die Fiktion einer Aufteilung steuerlicher Leistungsfähigkeit auf Individuen möglich und sinnvoll ist, halte ich die Argumente für eine Individualbesteuerung für überlegen; im folgenden werden daher Fragen der steuerlichen Gleichbehandlung im Vergleich zwischen Individuen behandelt. Aus dieser Sicht ist es wenig ergiebig, Steuerbelastungsvergleiche zwischen Haushalten gleicher Größe und gleichen Gesamteinkommens, jedoch unterschiedlicher Einkommensaufteilung, anzustellen, wie das in der Literatur zur Ehegattenbesteuerung üblich ist ${ }^{9}$.

Durch die Wahl des Individuums als Bezugseinheit der Besteuerung werden steuerliche Nachteile für einer Ehegemeinschaft bzw. Familie angehörende Individuen (z.B. im Falle einer Eheschließung) vermieden.

8 So legt auch die Royal Commission bei der Ableitung der Steuertarife für Individuen und Mehrpersonenhaushalte explizit eine individualorientierte Betrachtung zugrunde (Royal Commission on Taxation, 1966, Bd.3, 14-16).

9 Vgl. Andel (1980, 382-384), Brazer (1980, 226f.), Munnell (1980, 249). 
Damit wird das verfassungsrechtliche Gebot des Schutzes von Ehe und Familie erfüllt, so daß hierauf aufbauende steuerrechtliche Regelungen einer verfassungsgerichtlichen Überprüfung standhalten ${ }^{10}$. Diese Konzeption bewirkt eine Neutralität des Steuerrechts gegenüber allen denkbaren Formen des Getrennt- oder Zusammenlebens, die allerdings um die Berücksichtigung von lebensformbezogenen Faktoren, die die individuelle steuerliche Leistungsfähigkeit berühren (wie Unterhaltsverpflichtungen oder die sog. Haushaltsersparnis), zu ergänzen ist.

\section{Transfers zwischen Ehegatten (Ehegattenbesteuerung)}

\subsection{Grundsätzliche Problematik}

Traditionell finden sich Ausführungen zur Einkommensbesteuerung von Transfers zwischen Ehegatten unter der Bezeichnung "Ehegattenbesteuerung". Dabei werden Aspekte vermischt, die zum Teil nichts mit Transfers zwischen Ehegatten (z.B. die Frage des "Edukationseffekts"11), zum Teil auch nicht per se etwas mit ehelichen Lebensgemeinschaften zu tun haben (z.B. die Frage der Haushaltsersparnis). Im folgenden ist daher auf alle diese Aspekte einzugehen, da sie im Kontext einer Gesamtlösung für Transfers zwischen Ehegatten diskutiert werden.

\section{(1) Rechtliche Ausgangssituation}

Ausgangspunkt der Überlegungen zu der Frage, ob und in welcher Höhe Transfers zwischen den Ehegatten stattfinden und wie die einkommen-

10 Hier wird davon ausgegangen, daß es sich bei Artikel 6 GG um ein "Abwehrrecht" handelt, also lediglich eine Benachteiligung von Ehe und Familie ausgeschlossen werden muß, nicht jedoch eine Begünstigung für Ehe und Familie postuliert wird; vgl. Lang $(1983,117 f$.) sowie mit anderer Akzentuierung Birk (1983, 174f.).

11 Unter dem Stichwort "Edukationseffekt" wurde vor allem in den 50er Jahren die Absicht des Gesetzgebers diskutiert, durch die Ausgestaltung der Ehegattenbesteuerung Einfluß auf die Erwerbstätigkeit der Ehefrauen zu nehmen. Vgl. Bundesverfassungsgericht (1957, 79-82). 
steuerliche Reaktion hierauf ausfallen sollte, wird die rechtliche Stellung der Ehegatten zueinander sein, da die Ehe eine rechtliche Institution ist, deren unterschiedliche Ausgestaltung auch einkommensteuerliche Unterschiede zu anderen Lebensgemeinschaften begründet. Die Forderung, die Ehe steuerlich nicht zu berücksichtigen, paßt in eine andere (zivilrechtliche) Welt, jedenfalls aber nicht in die in der deutschen Lebenswirklichkeit anzutreffende.

Grundsätzlich besteht unabhängig vom gewählten Güterstand eine gegenseitige Verpflichtung der Ehegatten zur Zahlung von Unterhalt 12 . Im Rahmen des gesetzlich als Regelfall vorgesehenen Güterstandes der Zugewinngemeinschaft ( $\S 1363$ BGB) erfolgen Verfügungen über das Vermögen, insb. über das in die Ehe eingebrachte Vermögen, individuell, allerdings findet bei Auflösung der Ehe durch Scheidung eine hälftige Aufteilung der während der Ehe geschaffenen Vermögenswerte (Zugewinnausgleich) und Versorgungsanwartschaften (Versorgungsausgleich) statt (Schlüter, 1991, 75-88, 142-156; D.Schwab, 1991, 109-127, 179190); beim Tod eines der Ehegatten erfolgt ebenfalls ein Zugewinnausgleich, in der Regel jedoch in Form einer speziellen "erbrechtlichen Lösung", deren Konditionen die Grundidee nur verzerrt wiedergeben und für den überlebenden Ehegatten regelmäßig zu günstig ausfallen (Schlüter, 1991, 88-91; D.Schwab, 1991, 127-130). Durch Vereinbarung zwischen den Ehegatten kann der gesetzliche Güterstand der Zugewinngemeinschaft außer Kraft gesetzt und durch die "Gütergemeinschaft" oder "Gütertrennung", wobei letztere oft mit Nebenabsprachen insbesondere zum Versorgungsausgleich verbunden ist, ersetzt werden (Schlüter, 1991, 58-60; D.Schwab, 1991, 88-96).

Die tatsächliche Höhe von Transfers zwischen Ehegatten läßt sich von außen kaum objektiv feststellen, zumal wenn bei zusammenlebenden Ehegatten eine Erwerbs- und Verbrauchsgemeinschaft ${ }^{13}$ besteht und über

12 Diese Verpflichtung wird ggf. auch durch die Führung des gemeinsamen Haushalts erfüllt ( $\$ 1360$ BGB).

13 Vgl. z.B. Steuerreformkommission (1971, 192, Rz.554) sowie Lang $(1983,115)$, der sich auf die Verwendungsseite konzentriert und somit von einer Konsum- und Vermögensbildungsgemeinschaft spricht. 
die eheinternen Entscheidungsstrukturen wenig bekannt ist ${ }^{14}$. Da die Ehe in ihrer ökonomischen Bedeutung nicht objektivierbar ist, wenn die jeweilige individuelle Ausgestaltung durch die Ehepartner maßgeblich sein soll, wird für eine allgemeingültige Beschreibung die Ehe als Vertrag verstanden (D.Schwab, 1991, 17f.). Für die Beschreibung der Ehewirklichkeit stellt dies eine Fiktion dar, die jedoch dadurch gestützt wird, daß sie in Konfliktfällen auch zum Tragen kommt (Hackmann, 1979, 42).

Sollen die "Transfers steuerlicher Leistungsfähigkeit"15 zwischen Ehegatten innerhalb der Einkommensteuer Berücksichtigung finden, was immer dann gerechtfertigt ist, wenn von zwangsläufigen Verpflichtungen der Steuerpflichtigen ausgegangen wird, die Ehe selbst also nicht als einkommensteuerlich unerheblich angesehen wird, was schlecht zur grundgesetzlichen Verankerung der Institution Ehe passen würde ${ }^{16}$, dann ist von ihrer rechtlichen Typisierung auszugehen. Abweichend von der weitgehenden Vernachlässigung der rechtlichen Situation der Ehepartner zueinander durch die finanzwissenschaftliche Literatur ${ }^{17}$, halte ich diese Frage für den entscheidenden Ansatzpunkt ${ }^{18}$.

Die Pauschalierung innerehelicher Transferzahlungen anhand der rechtlichen Situation stellt dabei auch die für die Steuerpflichtigen günstigste Lösung dar. Würden stattdessen die - in der Praxis kaum ermittelbaren - tatsächlichen Transferzahlungen angesetzt, so führte dies bei modellhafter Betrachtung in jedem Fall zu einer Erhöhung der gemeinsamen Steuerschuld der Ehepartner: Entweder der tatsächliche

14 Zum eheinternen Verhalten bei Kaufentscheidungen vgl. Brandstätter/ Kirchler/Wagner (1987). Hackmann (1972/73, 512-518) beschränkt den eheinternen Einkommenstransfer.

15 So das Bundesverfassungsgericht $(1983,346)$ in seinem Urteil vom 3.11.1982. Siehe auch Lang $(1988,629)$.

16 Allerdings gibt es doch zu denken, wenn die steuerliche Behandlung der Ehegatten in vielen Fällen zum entscheidenden Motiv der Eheschließung wird.

17 Ausnahmen bilden Pohmer (1968, 147f., 165f.; 1985, 239, 246f.), Metze $(1982,797)$ sowie Brümmerhoff $(1990,314)$.

18 Damit wird - wie oben bereits erwähnt - die traditionelle Herangehensweise, die auf eine steuerliche Gleichbehandlung von Personen(gruppen) ohne Berücksichtigung der rechtlichen Umstände abzielt, abgelehnt. 
Umfang eheinterner Transfers bleibt unter der eherechtlich ableitbaren Höhe, dann müßte wegen der ungleichmäßigeren Verteilung der Steuerbemessungsgrundlage eine höhere Steuerprogression die Folge sein, oder der tatsächliche Transferumfang übersteigt die allein als zwangsläufig zu betrachtende eherechtlich ableitbare Höhe, dann müßte die "freiwillige Komponente" zusätzlich beim Empfänger versteuert werden.

\section{(2) Haushaltsersparnis und häusliche Dienste}

Im Falle gemeinsamer Haushaltsführung ist bei gleicher Versorgungsqualität für Teile des Bedarfs eine Reduktion der Pro-Kopf-Ausgaben möglich (Haushaltsersparnis). Auch kommt es in der Regel zu einem Austausch von häuslichen Diensten zwischen den Haushaltsmitgliedern.

Beide Aspekte spielen in der Diskussion um eine sachgerechte Ehegattenbesteuerung traditionell eine große Rolle und sind mit einigen Vorschlägen zur Ehegattenbesteuerung untrennbar verbunden. Meiner Ansicht nach sollten sie jedoch nicht im Zentrum von Reformvorschlägen stehen, da eine gemeinsame Haushaltsführung nicht das Charakteristikum der Ehe darstellt. Sie werden daher gesondert anzusprechen sein.

\section{(3) Aspekte der Steuerneutralität}

In bezug auf die Erwerbstätigkeit der Ehepartner wird gefordert, die Art der Ehegattenbesteuerung dürfe sich nicht auf die diesbezüglichen Entscheidungen der Ehepartner auswirken. Ein Anreiz zur Nichterwerbstätigkeit geht neben der Steuerfreiheit der Haushaltstätigkeit im eigenen Haushalt (Verzerrung der Einkommensteuer zugunsten aller "Freizeitaktivitäten") von den für einen Zweitverdiener bei allen Formen der Berücksichtigung der Eheschließung (z.B. gemeinsame Veranlagung, steuerliche Anerkennung von Transfers zwischen Ehegatten) im Vergleich zur individuellen Besteuerung des jeweiligen Erwerbseinkommens höheren Steuersätzen aus (Munnell, 1980, 263f.; P.B.Musgrave, 1981, 341343). Grundsätzlich treffen diese Effekte beide Ehepartner gleichermaßen: Der erste Effekt wirkt zuungunsten jeder Art steuerpflichtiger Tätigkeit, der zweite Effekt führt - in der deutlichsten Form der gemein- 
samen Veranlagung bzw. des vollen Einkommensausgleichs - zu gleichen marginalen Steuersätzen beider Ehegatten. Wenn allerdings davon ausgegangen werden kann, daß Hausarbeit traditionell von Frauen geleistet wird, daher von vornherein die Rolle des Zweitverdieners die Rolle der Frau ist, dann können steuerliche Regelungen, die auf einer partnerschaftlichen Eheauffassung beruhen, dazu beitragen, daß diese Rollenverteilung verfestigt wird. Eine Benachteiligung der Frau, die in der steuerlichen Regelung gesehen wird, liegt sehr viel tiefer begründet, nämlich bereits in der traditionellen Rollenverteilung, bei der Frauen über eine schlechtere Ausstattung mit am Arbeitsmarkt verwertbarem Humankapital verfügen (Galler/Ott, 1987, 403) und damit ein erhöhtes Risiko des Auseinanderbrechens einer Ehe tragen. Das eigentliche Ziel vieler Kritiker einer steuerlichen Berücksichtigung der Ehe ist daher nicht Steuerneutralität an sich, sondern ein möglichst hoher Grad an Gleichverteilung von Aufgaben zwischen den Ehepartnern, insbesondere Erwerbstätigkeit in gleichem Umfang, was eine größere Selbständigkeit ermöglicht. Hierzu können andere Politikbereiche aber entscheidendere Beiträge leisten als die Steuerpolitik (Galler/Ott, 1987), die möglichst durchgängig das Ziel der Besteuerung nach der Leistungsfähigkeit verfolgen sollte. Gleichwohl sind auch steuerliche Reaktionen möglich, die im einzelnen noch darzulegen sein werden ${ }^{19}$. Dabei sollte aber die Besteuerung nach der Leistungsfähigkeit nicht aufgegeben werden, wie dies unter dem Stichwort "equity-efficiency-trade-off" gefordert wird 20.

Hinzu kommt, daß sich das Ziel der Steuerneutralität für den Bereich der Ehegattenbesteuerung nicht widerspruchsfrei formulieren läßt. So steht die Neutralität gegenüber der Aufgabenverteilung in der Ehe, die eine von der internen Verteilung von Erwerbstätigkeit und Haushaltstätigkeit auf die Ehepartner unabhängige Steuerbelastung fordert, im Gegensatz zur oben angeführten Neutralität gegenüber der Erwerbstätigkeit der Ehegatten. Beide Arten von Neutralität sind, obwohl sie den Grund-

19 Vgl. Kapitel VI.3.2.3.2.(4) und VI.3.2.3.3.(3).

20 Dort steht die Differenzierung der Steuerbelastung nach der Arbeitsangebotselastizität im Mittelpunkt; vgl. Brazer (1980, 227f.) und P.B.Musgrave (1981, 346f.). 
gedanken einer effizienten Ressourcenallokation gemeinsam haben, nicht miteinander vereinbar (vgl. auch Hackmann, 1979, 41f.).

Schließlich ist, wenn von Neutralität die Rede ist, ein Aspekt steuerlicher Gerechtigkeit gemeint: Durch den Umstand der Eheschließung soll sich die Höhe der Steuerbelastung nicht ändern (Andel, 1980, 383; P.B.Musgrave, 1981, 346f.). Damit wäre gleichzeitig eine steuerliche Gleichbehandlung von Ledigen und Verheirateten erreicht. Eine solche Neutralität der Einkommensteuer macht jedoch nur dann Sinn, wenn sich durch eine Eheschließung an den steuerlich relevanten Verhältnissen der Beteiligten nichts ändert. Wegen der Erwerbs- bzw. Unterhaltsgemeinschaft zwischen Ehegatten ist das aber gerade nicht der Fall!

\subsection{Modelle der Ehegattenbesteuerung}

\subsubsection{Derzeitige Rechtslage}

Das geltende Einkommensteuerrecht sieht als Regelform der Ehegattenbesteuerung faktisch das Splitting mit dem Faktor 2 vor ( $\S \S 26(3), 26 \mathrm{~b}$, 32a(5) EStG), d.h. das gemeinsame Einkommen wird jedem der Ehegatten zur Hälfte zugerechnet. Damit wird eine Erwerbsgemeinschaft der Ehegatten unterstellt, was für den Regelfall zutreffend ist, jedoch den Fall der vereinbarten Gütertrennung nicht gesondert beachtet. Andererseits ist das Ehegattensplitting für dauernd getrennt lebende Ehepartner nicht wählbar, obwohl sich an deren rechtlichen Beziehungen im Vergleich zur sogenannten "intakten Ehe" (Lang, 1983, 113) im wesentlichen - abgesehen vom Wegfall der Haushaltsersparnis - nichts geändert hat 21 .

Daneben besteht eine Wahlmöglichkeit für die getrennte Veranlagung, die aber - von Sonderfällen abgesehen (Herrmann/Heuer/Raupach, 1974, Rz.20) - nicht genutzt wird, da sie regelmäßig zu einer höheren Steuerbelastung führt. Eine solche Wahlmöglichkeit widerspricht im übrigen der Forderung nach steuerlicher Gleichbehandlung gleicher Tatbestände.

21 Vgl. zur Entstehungsgeschichte dieser Bestimmung, die ursprünglich eine Schutzfunktion für Getrenntlebende hatte, Osthövener (1971, 57), zu einer kritischen Auseinandersetzung damit Osthövener (1971) und v.Mirbach (1987). 
Im geltenden Recht wird der Haushaltsersparnis von Mehrpersonenhaushalten nicht Rechnung getragen. Auch eine Berücksichtigung der Haushaltsproduktion erfolgt weder direkt noch indirekt über die Möglichkeit eines Werbungskosten- bzw. Betriebsausgabenabzugs von Ausgaben für die Haushaltsführung (Bundesfinanzhof, 1979; Ausnahmen in besonderen Fällen nach $\S \S 33,33 a(3), 33 b(6), 10(1)$ Nr.8 EStG).

Außerehelichen Unterhaltsbeziehungen kann nur durch Sonderregelungen Rechnung getragen werden, die systematisch nicht zur praktizierten Ehegattenbesteuerung passen. So besteht derzeit die Möglichkeit, Unterhaltszahlungen an den geschiedenen oder dauernd getrennt lebenden Ehegatten nach § 10(1) Nr.1 EStG bis zu einem Höchstbetrag von 27.000 DM als Sonderausgaben abzuziehen, sofern der Empfänger seine $\mathrm{Zu}$ stimmung erteilt und die entsprechenden Beträge nach § 22 Nr.1a EStG selbst versteuert. Parallel hierzu besteht die Option auf eine Berücksichtigung der Unterhaltszahlungen im Rahmen der außergewöhnlichen Belastungen des § $33 \mathrm{a}$ (1) EStG (Höchstbetrag 6.300 DM), wobei für die Höhe der anzuerkennenden Unterhaltszahlungen hier auch deren Zwangsläufigkeit ausschlaggebend ist 22 .

\subsubsection{Abgelehnte Alternativen: Gemeinsame Veranlagung ohne Splitting}

Die gemeinsame Veranlagung ohne Splitting macht keine Unterschiede zwischen einem Ehepaar und einer Einzelperson 23 - dem entspricht die Wahl des Haushalts als Bezugseinheit für Steuerbelastungsvergleiche, unabhängig von der Zahl der ihm angehörenden Personen. Auf das Individuum bezogen bedeutet eine solche Sichtweise, daß jeder Ehegatte einen halben Grundfreibetrag erhält, außerdem einer stärkeren Steuerprogression als eine Einzelperson unterworfen wird. Auch eine Modifikation durch Verdoppelung des Grundfreibetrags für Ehegatten berück-

22 Zum gesamten Konglomerat der derzeitigen einkommensteuerlichen Berücksichtigung von Unterhaltsverpflichtungen vgl. Tipke/Lang (1991, 382-389).

23 Das biblische Wort "ihr werdet eins" (Gen. 2,24) wird hier offensichtlich wörtlich genommen. 
sichtigt lediglich den ersten der beiden genannten Faktoren. Eine solche Praxis, die die Existenz zweier Personen weitgehend ignoriert, wäre allenfalls bei einer sehr extremen Einschätzung der Haushaltsersparnis vertretbar. Dieses Verfahren wird daher nicht weiter verfolgt; es wird auch in der Literatur einmütig abgelehnt, wozu nicht zuletzt die Entscheidung des Bundesverfassungsgerichts (1957) beigetragen hat.

\subsubsection{Befürwortete Alternativen}

\subsubsection{Vorbemerkungen}

Gemeinsam ist den hier befürworteten Alternativen, daß sie eine Bezugnahme auf das Individuum ermöglichen, was sowohl aus konzeptionellen Gründen als auch aus Gründen der Praktikabilität wegen des Wechsels der Zugehörigkeit von Individuen zu Haushalten angestrebt wird. Dies bildet gleichzeitig die Basis für eine systemgerechte Integration außerehelicher Unterhaltsbeziehungen.

Weiterhin wird die rechtliche Situation als Grundlage der Zuordnung steuerlicher Leistungsfähigkeit auf die beteiligten Personen (Ehepartner) herangezogen. Daher wird die steuerliche Behandlung der Ehepartner nach der getroffenen Vereinbarung über den ehelichen Güterstand differenziert. Reformmaßnahmen, die auf grundlegende Änderungen der Ehegattenbesteuerung abzielen, haben demzufolge zunächst an den bürgerlich-rechtlichen Regelungen anzusetzen. Dem entspricht auch die Forderung des Bundesverfassungsgerichts (1983, 346), dem Transfer steuerlicher Leistungsfähigkeit zwischen den Ehegatten Rechnung zu tragen. Ebenfalls wird eine Benachteiligung der Ehe (Bundesverfassungsgericht, 1957, 55) durch die hier vorgetragenen Alternativen ausgeschlossen.

Die Grundmodelle der Ehegattenbesteuerung können durch eine Berücksichtigung von Haushaltsersparnis und Haushaltsproduktion ergänzt werden. Eine solche Ergänzung ist prinzipiell eheunabhängig möglich, kann also für alle betroffenen Haushalte - auch für nichteheliche Lebensgemeinschaften - durchgeführt werden. 
Wenn im folgenden nach dem gesetzlichen Güterstand differenziert wird, wird vorausgesetzt, daß sog. Mischgüterstände nicht zulässig sind und daher nicht vorkommen. Im allgemeinen ist dies zwar zutreffend, jedoch können die Ehegatten im Rahmen der Vertragsfreiheit bestimmte abweichende Regelungen treffen, auch ist in Sonderfällen die Wahl des Güterstandes nach ausländischem Recht möglich (Schlüter, 1991, 58-60; D.Schwab, 1991, 94-96). Aus verwaltungstechnischen Gründen sollte für einkommensteuerliche Zwecke nur zwischen den gesetzlich vorgesehenen Güterständen unterschieden werden; in Zweifelsfällen durch selbstgewählte Abweichungen der Ehepartner sollte die individualisiertere Variante und damit die wegen der Steuerprogression aus Sicht der Steuerpflichtigen nächstschlechtere Alternative zur Anwendung kommen. Im übrigen ist der gewählte Güterstand dadurch nachprüfbar, daß Eheverträge mit dem Ziel der Abweichung vom gesetzlichen Güterstand der Zugewinngemeinschaft der notariellen Beurkundung bedürfen (D.Schwab, 1991, 94). Gegebenenfalls könnte zusätzlich eine Eintragungspflicht in das Güterrechtsregister eingeführt werden.

\subsubsection{Grundformen}

\section{(1) Güterstand der Gütergemeinschaft}

In der Gütergemeinschaft kommt am deutlichsten das Leitbild einer gemeinschaftsorientierten Eheauffassung zum Ausdruck ${ }^{24}$. Die ökonomischen Verfügungsmöglichkeiten über das gemeinsame Anfangsvermögen und, was wichtiger ist, über den Zugewinn sind völlig gleichmäßig auf die Ehepartner verteilt. Die sonst notwendige Trennung zwischen in die Ehe eingebrachtem Vermögen, das in der individuellen Zuordnung durch die Eheschließung nicht angetastet wird, während der Ehe gebildetem Vermögen sowie den Einkünften (inkl. allen Vermögenseinkünften), aus denen der gemeinsame Lebensunterhalt zu bestreiten ist und auf die sich die Unterhaltsansprüche der Ehegatten beziehen, entfällt hier. Die faktische hälftige Aufteilung des Anfangsvermögens verlangt hier allerdings

24 Vgl. dazu auch Karl-Bräuer-Institut des Bundes der Steuerzahler (1971, 142-144). 
besondere Beachtung, da sie mit einem entsprechenden Vermögenstransfer zwischen den Ehepartnern verbunden ist. Güterrechtlich wird die Ehe mit einer Rückwirkung auf vor der Ehe erwirtschaftetes Einkommen ausgestattet, sofern daraus Vermögen gebildet wurde.

Offensichtlich entspricht es dieser güterrechtlichen Konstellation und den damit verbundenen individuellen Verfügungsmöglichkeiten, wenn für einkommensteuerliche Zwecke jedem der Ehepartner die Hälfte des gemeinsamen Einkommens zugerechnet wird. Steuertechnisch wird dies sowohl durch eine getrennte Veranlagung der Ehepartner bei Korrektur der individuellen Bemessungsgrundlagen um die entsprechenden innerehelichen Transfers durch das Verfahren des sog. Realsplitting (Lang, 1983, 110-112, 123-125) als auch durch eine gemeinsame Veranlagung mit Splitting 25 realisiert. Beide Verfahren führen zum gleichen Ergebnis, die Hälfte des gemeinsamen Einkommens wird jeweils dem Grundtarif unterworfen. Auch bei der Ehegattenbesteuerung wird letztlich nicht vom Grundsatz der Individualbesteuerung abgewichen, was bedeutet, daß für alle Steuerpflichtigen der gleiche Maßstab - ausgedrückt im Tarif verwendet werden kann. Das hat zur Folge, daß eine Diskriminierung der Eheschließung vermieden wird. Das Verfahren des Realsplitting ist jedoch in der Ausgestaltung transparenter, und auch andere zwangsläufige Unterhaltszahlungen (z.B. zwischen geschiedenen Ehepartnern) lassen sich - anders als beim Divisorensplitting - problemlos in das System integrieren 26 . Die individuelle Steuerbemessungsgrundlage drückt dann die

25 Dabei wird zunächst vom Grundmodell des Splitting mit Faktor 2 ausgegangen; dieses kann zur Berücksichtigung einer Haushaltsersparnis, auf die später noch zurückzukommen sein wird, über eine Reduktion des Splittingfaktors modifiziert werden.

26 Pohmer $(1985,247)$ hält dagegen das Realsplitting für komplizierter, weil es beim Empfänger von Transferzahlungen eine Steuerpflicht auslöst. Gerade diese individuelle Betrachtung steuerlicher Leistungsfähigkeit begründet jedoch die Eignung des Realsplitting als generelles Prinzip der Besteuerung für Fälle zwangsläufiger Unterhaltsverpflichtungen. Zudem tritt eine Komplizierung in den Fällen, in denen ein Divisorensplitting anwendbar ist (nämlich für Ehepartner und ggf. deren Kinder), nicht ein, da hier die Fiktion eines Realsplitting im Rahmen der Steuerveranlagung genügt. 
jeweiligen tatsächlichen Verfügungsmöglichkeiten über Einkommen, normiert anhand der rechtlichen Situation, aus.

Als ideal zum Ehegattensplitting passender Güterstand ist allerdings nicht die Gütergemeinschaft, sondern die Errungenschaftsgemeinschaft zu nennen, bei der zwar die Anfangsvermögen getrennt bleiben, aber eine sofortige gemeinsame Verfügungsberechtigung über den Zugewinn besteht (Gernhuber, 1980, 432f., 440; D.Schwab, 1991, 95, Rz.187).

Innerhalb der vorgetragenen Argumentation ist es völlig unerheblich, ob es sich um eine sog. intakte Ehe handelt oder nicht, insb. ob die Ehepartner einen gemeinsamen Haushalt führen oder dauernd getrennt leben, da sich an der Erwerbsgemeinschaft der Eheleute hierdurch im Grundsatz nichts ändert 27 . Im Konfliktfall der "nicht intakten Ehe" ist es sogar sehr viel wahrscheinlicher, daß die güterrechtlichen Bestimmungen des Bürgerlichen Gesetzbuchs in der Ehewirklichkeit voll umgesetzt werden.

\section{(2) Güterstand der Zugewinngemeinschaft}

Im Güterstand der Zugewinngemeinschaft bestehen während der Dauer der Ehe lediglich Unterhaltsansprüche zwischen den Ehepartnern in Form einer Verpflichtung der vollen Teilhabe des Ehepartners am gewählten Lebensstandard, der sich im Konsum ausdrückt (Schlüter, 1991, 42-48). Auch während der Ehe gebildetes Vermögen kommt wegen des Zugewinnausgleichs bei Auflösung der Ehe beiden Partnern gleichermaßen zugute; in die Ehe eingebrachtes Vermögen verbleibt im individuellen Besitz des jeweiligen Ehepartners.

Für die Zeit der Ehe und das während dieser Zeit erzielte Einkommen kommt die Zugewinngemeinschaft der Gütergemeinschaft sehr nahe, da letztlich - nach erfolgtem Ausgleich des während der Ehe erzielten $\mathrm{Zu}$ gewinns - auch hier von einer hälftigen Aufteilung des Einkommens ausgegangen werden muß: Die Aufwendungen für den Konsum werden sofort, diejenigen für Ersparnis später hälftig geteilt. Die formal beste-

27 Vgl. v.Mirbach (1987, 322). Lang (1983, 112-115) betont dagegen die Bedeutung der "intakten Durchschnittsehe". 
hende Vermögenstrennung wird durch die Regelungen zum ehelichen Unterhalt, zum Zugewinn- und Versorgungsausgleich faktisch ausgehebelt. Gestützt wird diese Auffassung auch durch die Existenz von Verfügungsbeschränkungen, die eine Sicherung der Forderung auf Zugewinnausgleich zum Ziel haben und bewirken, daß der rechtliche Status der Zugewinngemeinschaft bereits bei bestehender Ehe zur Geltung kommt und somit in die bestehende Ehe hineinwirkt ${ }^{28}$. Während der Ehe gebildetes Vermögen ist von Anfang an mit "Zugewinnausgleichsforderungen" des anderen Ehegatten belastet 29 .

Daher sollte die Zugewinngemeinschaft einkommensteuerlich wie die Gütergemeinschaft (hälftige Aufteilung des Gesamteinkommens) behandelt werden. Besondere Regelungen für die Eheschließung oder die Beendigung der Ehe sind hier nicht notwendig, da der Zugewinngemeinschaft vollkommen mit der hälftigen Aufteilung des Einkommens während der Ehezeit - und nur für diese Zeit - Rechnung getragen wird.

Die einzige verfassungsrechtlich haltbare Alternative zur hälftigen Aufteilung des Einkommens dürfte die Normierung von innerehelichen Transfers in Höhe der Unterhaltsverpflichtungen sein, wie sie für den Streitfall üblicherweise gerichtlich festgesetzt werden. Diese werden i.d.R. auf der Grundlage von Quoten berechnet, die bei Nichterwerbstätigkeit des unterhaltsverpflichteten Ehepartners ebenfalls 50\% betragen, bei Erwerbstätigkeit des Unterhaltsverpflichteten je nach zuständigem Oberlandesgericht 2/5 oder 3/7 der Einkommensdifferenz der Ehepartner, um einen Arbeitsanreiz in angemessener Höhe zu erhalten und einen Ausgleich für schwer quantifizierbaren berufsbedingten Mehrbedarf zu schaffen ${ }^{30}$. Bei hohen Einkommen, die nicht vollständig zur Deckung des Lebensbedarfs nach dem erreichten Lebensstandard eingesetzt werden müssen, sondern auch Spielräume für eine Vermögensbildung lassen,

28 So auch das Bundesverfassungsgericht $(1983,346)$ in seinem Urteil vom 3.11.1982. Vgl. ebenfalls Schlüter (1991, 64-75) und D.Schwab (1991, 98-109). Anderer Ansicht ist Mennel (1988, 91).

29 Vgl. Lang (1983, 114); anderer Ansicht Littmann (1968, 184).

30 Vgl. Kalthoener/Büttner (1989, 1-65). Die beiden unterschiedlichen Sätze (3/7 und 2/5) nähern sich wegen der im ersten Fall üblichen Berücksichtigung einer Werbungskostenpauschale in der Praxis noch weiter an. 
liegt allerdings der Unterhaltsanspruch unterhalb dieser am Einkommen orientierten Quoten (Kalthoener/Büttner, 1989, 65-67; D.Schwab, 1991, 165). Wegen der Nähe zu 50\%, der normalerweise bestehenden Verbrauchsgemeinschaft der Ehegatten und der mit der beschriebenen Lösung abgedeckten sonst nicht berücksichtigungsfähigen berufsbedingten Aufwendungen halte ich eine Orientierung an diesen Größen jedoch für weniger überzeugend. Hinzu kommt, daß eine solche Aufteilung des laufenden Einkommens nicht mit der weiterhin geltenden 50\%-Beteiligung bei Zugewinn- und Versorgungsausgleich kompatibel ist: In diesen Fällen müßte die ursprüngliche Einkommensaufteilung dann korrigiert werden, was in steuersystematisch sinnvoller Weise nicht mehr möglich ist. Eine entsprechende Berücksichtigung empfiehlt sich daher bereits zum Zeitpunkt der Entstehung der Ansprüche. Auch sind die Differenzen beider Verfahren nicht sehr groß, in einem Teil der Fälle (Erwerbslosigkeit des Unterhaltsverpflichteten) sogar Null.

Bei der hälftigen Zurechnung des Gesamteinkommens treten dann konzeptionelle Probleme auf, wenn, wie im Rahmen der derzeitigen Einkommensteuer, die Einkommensteuer dem Nominalwertprinzip folgt, dem Zugewinnausgleich jedoch ein Realwertkonzept zugrunde liegt (D.Schwab, 1989, 1114-1117; 1991, 115f.). Die lediglich die Inflation widerspiegelnden Vermögenseinkünfte werden dann fälschlicherweise zur Hälfte dem nicht vermögenden Ehepartner zugerechnet, was tendenziell einen niedrigeren Steuersatz, aber auch eine Beteiligung des nicht vermögenden Ehepartners an der inflationsbedingten steuerlichen Überbelastung zur Folge hat. Auch Vorschläge, die die Vermögenseinkünfte steuerlich individuell dem Vermögenseigner zuordnen (Tipke, 1984, 127; Albers, 1988, 418), bieten hier jedenfalls keine Lösung, da die realen Einkünfte beiden Ehepartnern zustehen.

\section{(3) Güterstand der Gütertrennung}

Der entscheidende Unterschied zu den beiden bislang genannten Güterständen liegt bei der Gütertrennung darin, daß nicht nur das vor der Ehe, sondern auch das während der Ehe gebildete Vermögen der Ehepartner getrennt bleibt. Ausgleichsforderungen auf das Vermögen treten hier bei 
Beendigung der Ehe nicht ein 31 . Es besteht allerdings auch hier ein voller Unterhaltsanspruch im Sinne einer Teilhabe am gemeinsamen Lebensstandard, der sich im Konsum ausdrückt. Jedoch läßt der Umstand der Gütertrennung auch den Schluß auf eine asymmetrische Verteilung von Konsumentscheidungsbefugnissen innerhalb der Ehe zu. Offenbar ist hier eine individualorientierte Auffassung der Ehe sehr viel stärker ausgeprägt $^{32}$.

Die Höhe von Unterhaltsansprüchen läßt sich wiederum aus für den Konfliktfall vorgesehenen Regelungen ableiten. Sie entspricht derjenigen Höhe, die bereits für den Fall der Zugewinngemeinschaft dargestellt wurde (i.d.R. ausgehend von $2 / 5$ oder $3 / 7$ bzw. $50 \%$ der Einkommensdifferenz). Allerdings erhält hier das Außerachtlassen der gesparten Einkommensteile ein anderes Gewicht, da bei Gütertrennung kein Zugewinnausgleich stattfindet. Eine Pauschalierung von Unterhaltsansprüchen ist wegen der Abhängigkeit von der individuellen Ersparnis nur schwer möglich. Wegen der expliziten Entscheidung der Ehepartner für eine Gütertrennung läßt sich hier eine Pauschalierung innerehelicher Transfers vertreten, die auf eine Absicherung des Existenzminimums des Ehepartners beschränkt bleibt 33 . Sollte eine solche Regelung als zu restriktiv empfunden werden, wäre auch die Festlegung eines darüber liegenden absolut fixen Höchstbetrags möglich, womit allerdings durch Einführung eines weiteren Normbetrags das Steuerrecht unübersichtlicher würde. Sofern gerichtlich festgesetzte Unterhaltsansprüche (im Fall der Trennung) nachgewiesen würden, wären diese anzuerkennen: Sie hätten auch deshalb eine andere Qualität, weil dann Unterhalt nicht mehr vorwiegend in Naturalien, sondern als für den Empfänger frei verfügbare monetäre Transferzahlung zu leisten ist.

31 Daher wird von der Vereinbarung eines Ausschlusses des Versorgungsausgleichs auch auf den Willen zur Gütertrennung geschlossen, allerdings nicht umgekehrt (Schlüter, 1991, 92f.).

32 Dies gilt auch, wenn die Gütertrennung aus Gründen gewählt wurde, die nicht im Innenverhältnis der Ehepartner liegen, z.B. aus Gründen der Haftungsbeschränkung.

33 So ist wohl auch Tipke $(1984,127)$ zu interpretieren. Lang $(1983,114)$ hingegen hält diese Konsequenz für zu hart. 
Die einkommensteuerliche Behandlung der Ehepartner bei Gütertrennung ginge also von den individuellen Bemessungsgrundlagen aus, die in pauschalierter Form um Unterhaltsverpflichtungen korrigiert würden. Dies führt i.d.R. nicht zu einer Angleichung der einkommensteuerlichen Bemessungsgrundlagen der Ehepartner.

\section{(4) Familienphasenspezifische Regelung des Güterstandes?}

In der Zunahme der Erwerbstätigenehen drückt sich der zunehmende Wille und die zunehmende Möglichkeit der Ehepartner aus, Verantwortung für ihre individuellen wirtschaftlichen Belange zu übernehmen. Sofern keine Kinder zu versorgen sind, stellt die Erwerbstätigenehe inzwischen den Regelfall dar; die früher übliche Hausfrauenehe hat als Leitbild weitgehend ausgedient ${ }^{34}$. Dies ist auch die Folge einer gewandelten Vorstellung von der Rolle der Frau in der Gesellschaft; Gemeinsamkeit und Gleichheit der Ehepartner unter patriarchalischer Führung werden in zunehmendem Maße durch Eigenverantwortung der Ehepartner abgelöst.

Es ist keine zwingende Konsequenz der Existenz von Erwerbstätigenehen, Änderungen des ehelichen Güterrechts und in deren Gefolge der Ehegattenbesteuerung vorzusehen, jedoch sind entsprechende Überlegungen naheliegend, da sie unter Umständen sehr viel besser den persönlichen Lebensgestaltungen der Ehepartner Rechnung tragen können. Auch könnte die rechtliche Situation ehelicher und nichtehelicher Lebensgemeinschaften einander angenähert werden 35 . Denkbar wäre es, von einer individuellen wirtschaftlichen Verantwortung der Ehepartner auszugehen und gleichzeitig Unterhaltsverpflichtungen der Ehegatten auf

34 So liegen die Erwerbsquoten der verheirateten Frauen unter 50 Jahren deutlich über 50\%, obwohl in der Bezugsbasis auch diejenigen Frauen enthalten sind, die Kinder betreuen und daher eine relativ geringe Erwerbsquote aufweisen (Statistisches Bundesamt, 1991, 113, 122). Ramm (1985, 270-274) fordert daher eine Typisierung des Eherechts, die insbesondere auch den Lebensumständen von Erwerbstätigenehen Rechnung trägt.

35 Dies erscheint schon deshalb als angemessen, weil im Empfinden der Bevölkerung das Steuerrecht Vorteile für die Ehe bringt. 
eine gegenseitige Absicherung des Existenzminimums (oder eines darüber liegenden fixen Betrages) und damit auf besondere Fälle zu beschränken. Damit würde auch die letzte ökonomische Bastion des oben skizzierten "Familienmodells" durch eine individuelle materielle Absicherung weitgehend ersetzt.

Einkommensteuerliche Konsequenz wäre eine reine Individualbesteuerung, allerdings unter Berücksichtigung eventueller geleisteter oder empfangener Unterhaltszahlungen im beschriebenen beschränkten Umfang. Der Kritik an den möglichen Wirkungen der Ehegattenbesteuerung auf die Erwerbstätigkeit der Ehepartner ("Zweitverdienereffekt") könnte mit einem solchen Vorschlag weitgehend Rechnung getragen werden (allerdings wohl erst bei einer Übernahme als Regel-Güterstand). Möglichen Steuermanipulationen könnte durch eine Dokumentation der Vermögensverhältnisse gegengesteuert werden 36 .

Eine solche güterrechtliche Regelung sollte für Zeiten der Kindererziehung und -betreuung ergänzt werden, da dann kaum noch von einer Erwerbstätigkeit beider Ehepartner ausgegangen werden kann. Eine Kombination des hier präsentierten Güterstandes mit dem der Zugewinngemeinschaft (bzw. der Gütertrennung) bietet sich an, wobei die $\mathrm{Zu}$ gewinngemeinschaft bis zur Wiederaufnahme der vollen Erwerbstätigkeit des kinderbetreuenden Elternteils bzw. maximal bis zu einem bestimmten Lebensalter (z.B. dem 15.Lebensjahr ${ }^{37}$ ) des Kindes zur Geltung käme (familienphasenspezifische Regelung). Damit wäre eine recht gute Anpassung an die tatsächliche Lebenssituation der Ehepartner möglich. Indirekte materielle Nachteile, die mit dem vorübergehenden Aufgeben einer Erwerbstätigkeit verbunden sind (Verlust an Humankapital und Aufstiegschancen), könnten allerdings kaum berücksichtigt werden; diese wären

36 Es verbleiben allerdings für die Gruppe der Selbständigen gewisse Möglichkeiten zu Scheinarbeitsverhältnissen. Vgl. dazu insgesamt Kapitel VI.5.

37 Die Erwerbsquoten von Frauen mit Kindern steigen bereits ab dem 7. Lebensjahr von Kindern deutlich an und erreichen ab dem 15. Lebensjahr ihr Maximum (Statistisches Bundesamt, 1991, 122). Auch der 4. Familienbericht der Bundesregierung (Bundesminister für Jugend, Familie, Frauen und Gesundheit, 1986, 36f.) geht für die Notwendigkeit einer Kinderbetreuung vom 15. Lebensjahr als Obergrenze aus. 
durch begleitende staatliche und tarifvertragliche Maßnahmen in Grenzen zu halten (z.B. Wiedereinstellungsgarantien, organisierte Fortbildungsmaßnahmen, stärkere Bereitstellung von Teilzeitarbeitsplätzen).

\subsubsection{Ergänzungen}

\section{(1) Berücksichtigung der Haushaltsersparnis}

Das Argument der Haushaltsersparnis zielt darauf ab, daß sich bei gemeinsamer Haushaltsführung mehrerer Personen ein gegebener Bedarf kostengünstiger befriedigen läßt (Haller, 1981a, 72). Die Ursachen einer solchen Kostendegression liegen vor allem in der gemeinsamen Nutzung von Teilen der Wohnung und privaten Gebrauchsgütern sowie bei Vorteilen in der haushaltsinternen Produktion von Dienstleistungen. Die Haushaltsersparnis knüpft an einen gemeinsamen Haushalt an, ist daher nicht auf Ehen beschränkt, für die eine gemeinsame Haushaltsführung zwar typisch, aber nicht notwendig ist, sondern gilt auch für andere Formen gemeinsamen Haushaltens.

Es ist allerdings zu bedenken, daß die Qualität der Bedürfnisbefriedigung z.B. wegen der notwendigen Kompromißbildung oder eingeschränkter zeitlicher Verfügbarkeit von Gebrauchsgütern abnehmen kann. Mit steigendem Lebensstandard dürfte die relative Bedeutung der Haushaltsersparnis wegen der zunehmenden Bedeutung der Qualitätskomponente, die sich bei Gebrauchsgütern in der jederzeitigen individuellen Verfügbarkeit ausdrückt, sowie der Verlagerung von Dienstleistungen aus dem Haushalt heraus sehr stark abnehmen 38 .

Aus einer - hier allerdings nur ergänzend heranzuziehenden nutzenorientierten Betrachtungsweise heraus läßt sich sogar sagen, daß für einen marginalen Haushalt die Vorteile gemeinsamer Haushalts-

38 Beispiele sind hier im Bereich der Gebrauchsgüter Zweitwagen, individuelle Zimmer, mehrere Fernsehgeräte usw., im Bereich der Dienstleistungen der Kauf von Fertiggerichten bzw. Besuch von Restaurants. Vgl. auch Haller (1981b, 16). Bei einem gegebenen Warenkorb, der letztlich homogene Präferenzen unterstellt, wird die Haushaltsersparnis allerdings immer von Gewicht bleiben. 
führung gleich Null sein müssen; andernfalls würden Nutzenabwägungen dazu führen, daß sich weitere Einzelpersonen zu gemeinsamen Haushalten zusammenschlössen.

Objektivierbar ist, daß ein gegebener Bedarfskatalog für eine in einem gemeinsamen Haushalt lebende zweite Person kostengünstiger zu befriedigen ist wie für eine in einem Einzelhaushalt lebende Person (so auch die Abstufung der Sozialhilfe-Regelsätze). Der Ort einer Berücksichtigung von persönlichem Bedarf innerhalb der Einkommensteuer ist zunächst der Existenzminimum-Grundfreibetrag, dessen Ermittlung sich bei Anlehnung an die Sozialhilfe auf einen gegebenen Warenkorb stützt. Dementsprechend ist es konzeptionell richtig, den Grundfreibetrag für eine zweite und jede weitere in einem gemeinsamen Haushalt lebende Person zu kürzen (Lang, 1983, 115; Leibfritz/Parsche, 1988, 151).

Steuertechnisch bereitet die direkte Durchsetzung dieser Forderung, d.h. die Reduktion des Grundfreibetrags für Haushaltsangehörige, jedoch Probleme, da willkürlich festgelegt werden muß, wer die erste im Haushalt lebende Person und wer die weiteren Personen sind ${ }^{39}$, sowohl für einen eventuellen Lohnsteuerabzug als auch für eine Einkommensteuerveranlagung. Da offensichtlich alle im Haushalt lebenden Personen einen Vorteil aus der Haushaltsersparnis ziehen, ist es unter Gleichbehandlungsaspekten naheliegend, die Haushaltsersparnis gleichmäßig bei allen (erwachsenen ${ }^{40}$ ) Haushaltsmitgliedern zu berücksichtigen. Dann müßte jedoch der individuelle Grundfreibetrag nach der Haushaltsgröße differieren, was zu einer erheblichen Komplizierung des Steuererhebungsverfahrens (bei Änderung der Haushaltsgröße müßten die Angaben bei allen Beteiligten revidiert werden!) führen würde.

39 Unproblematisch wäre in dieser Hinsicht lediglich eine gemeinsame Veranlagung mit dem Haushaltseinkommen, wozu oben bereits für Ehegatten kritisch Stellung genommen wurde, die zudem für andere Haushaltsgemeinschaften völlig undenkbar ist.

40 Denkbar ist auch eine Berücksichtigung der mit steigender Personenzahl zunehmenden Haushaltsersparnis beim Bedarf von Kindern (i.d.R.: Kinderfreibetrag), was aber bei ohnehin schon nach dem Alter der Kinder differierenden Beträgen für die Steuerpflichtigen kaum noch zu durchschauen ist. Vgl. hierzu auch Kapitel VI.4.1.3. 
Alternativ bietet sich die indirekte Vorgehensweise an, bei der Festlegung des Grundfreibetrages von einem Zwei-Erwachsenen-Haushalt auszugehen. Die Haushaltsersparnis ist dann für diesen Fall bereits berücksichtigt, Alleinlebenden ist ein zusätzlicher Freibetrag zum Ausgleich der bei ihnen nicht vorhandenen Haushaltsersparnis zu gewähren 41 . Dies hätte den Vorteil, daß lediglich bei Alleinlebenden ein zusätzlicher Freibetrag zu berücksichtigen ist (durch Eintragung auf der Lohnsteuerkarte bzw. im Veranlagungsverfahren), wie es heute bereits bei anderen Sachverhalten üblich ist. Nachteilig ist, daß größere Haushalte von einem solchen Verfahren profitieren würden 42 , was aber angesichts der geringen Zahl der betroffenen Personen, der in einem vertretbaren Rahmen bleibenden Steuerunterschiede und der Vorteile der Steuervereinfachung in Kauf genommen werden kann.

Ein solcher Sonderfreibetrag ist auch bei Vorliegen eines Haushalts aus einem Erwachsenen und Kindern ("Alleinerziehenden-Haushalt") einzuräumen, da für Kinder ohnehin schon ein reduzierter Grundfreibetrag üblich ist ${ }^{43}$.

Bei Anwendung einer solchen Lösung läßt sich die Haushaltsersparnis aller Mehrpersonenhaushalte berücksichtigen, eine Beschränkung auf (zusammenlebende) Ehepaare ist hier nicht notwendig. Da die hier in Rechnung gestellte Haushaltsersparnis vor allem mit der gemeinsamen Nutzung von Teilen der Wohnung und einer Grundausstattung an Gebrauchsgegenständen begründet wird, daher allein die Wohngemein-

41 So, allerdings ohne die beschriebenen konzeptionellen Probleme zu diskutieren, auch Andel $(1980,385)$ und Haller (1981b, 18-20).

42 Angenommen, der Existenzminimum-Grundfreibetrag für einen Alleinlebenden solle 10.000 DM, derjenige für weitere im Haushalt lebende Personen 8.000 DM betragen. Dann errechnet sich ein "Normal"-Grundfreibetrag von $9.000 \mathrm{DM}$, der für Alleinlebende durch einen Sonderfreibetrag von 1.000 DM aufgestockt wird. Für einen 4-Erwachsenen-Haushalt läge der konzeptionell richtige Grundfreibetrag bei $8.500 \mathrm{DM}$ pro Person, bei dem geschilderten vereinfachten Verfahren könnten allerdings 9.000 DM pro Person geltend gemacht werden.

43 Vgl. die Höhe von Kinderfreibeträgen und Kindergeld sowie die Regelsätze nach dem Bundessozialhilfegesetz, die zur Orientierung dienen sollten. 
schaft und nicht eine darüber hinausgehende Lebens- und Wirtschaftsgemeinschaft ausschlaggebend ist, ist eine restriktive Definition des Alleinlebenden-Haushalts angebracht: alle Arten von Wohngemeinschaften sind in diesem Sinne als Mehrpersonen-Haushalte zu werten ${ }^{44}$. Steuertechnisch ist das Vorliegen eines Alleinlebenden-Haushalts durch eine entsprechende Erklärung des Steuerpflichtigen in der Einkommensteuerveranlagung bzw. im Lohnsteuerjahresausgleich festzustellen, einen zusätzlichen Nachweis könnte im Regelfall bereits die Lohnsteuerkarte, die ja vom Einwohnermeldeamt ausgestellt wird, liefern. Durch die restriktive Definition des Alleinlebenden-Haushalts dürften Kontrollen, wie sie z.Zt. im Rahmen der Sozialhilfe zur Feststellung einer Haushaltsgemeinschaft vorgenommen werden und Ärgernis erregen (Brühl, 1989, 154-157), überflüssig sein. Größenordnungsmäßig errechnet sich für das Jahr 1992 ein Sonderfreibetrag in Höhe von 1000 DM pro Jahr ${ }^{45}$.

Denkbar wäre es auch, eine mit steigendem Einkommen zunehmende Haushaltsersparnis von Mehrpersonenhaushalten anzusetzen. Dem entspricht die Modifikation des Ehegattensplitting durch Reduktion des Splittingfaktors, d.h. die Wahl eines Faktors kleiner als 2 (Wissenschaftlicher Beirat beim BMF, 1967, 349; Haller, 1981b, 16f.). Wird dieser Faktor unabhängig von der Einkommenshöhe konstant gehalten, dann drückt er eine zum Haushaltseinkommen proportionale Haushaltsersparnis aus, was ansteigenden Absolutbeträgen entspricht.

Rein formal erfordert dies eine gemeinsame Veranlagung der betrachteten Mehrpersonenhaushalte, was auf unüberwindbare Schwierigkeiten bei allen nichtehelichen Lebensgemeinschaften stößt (vgl. §§ 44, 268-280 AO; Pump, 1987). Aus verschiedenen Gründen ist die Annahme steigender Haushaltsersparnis jedoch auch konzeptionell fragwürdig: Es dürfte nur auf die gemeinsame konsumtive Einkommensverwendung Bezug genommen werden, was im Hinblick auf die Ersparnis, vor allem

44 Dagegen werden in der amtlichen Statistik erst zusammenwohnende und gemeinsam wirtschaftende Personen als Haushalt erfaßt (Bretz/Niemeyer, 1992, 73f.).

$45 \mathrm{Vgl}$. die Zahlenangaben und -auswertungen bei Karl-Bräuer-Institut (1991, 47-53). 
aber auf die verschiedenen Formen von Haushaltsgemeinschaften fragwürdig wäre. Bei einem gemeinsamen Haushalt zweier Steuerpflichtiger mit stark differierenden Einkommen läßt sich wohl kaum ohne weiteres von einer Haushaltsersparnis durch gemeinsame Verwendung des Gesamteinkommens ausgehen. Je höher die Ansprüche an die gemeinsame Wirtschaftsführung der im Haushalt lebenden Personen, desto restriktiver ist der in Frage kommende Personenkreis zu fassen. Entsprechend bezieht sich der Vorschlag des Splitting mit reduziertem Splittingfaktor - von der Ausweitung um im Haushalt lebende Kinder als Familiensplitting abgesehen - auch nur auf Ehegatten. Dieses Verfahren umfaßt einen großen, aber tendenziell rückläufigen, Anteil der Mehrpersonen-Haushalte, wird daher der vorhandenen Vielfalt der Lebensformen nicht gerecht und ist wegen der Beschränkung auf Ehegatten verfassungsrechtlich bedenklich 46 .

Hinzu kommt - und dieses Argument ist letztlich ausschlaggebend -, daß wegen des Fehlens eines "Warenkorbs" wie beim Grundbedarf die Abschätzung einer Haushaltsersparnis noch stärker auf subjektiven Einschätzungen der Nutzen der im Haushalt lebenden Personen beruhen müßte, was im Grenzfall die Berücksichtigung einer Haushaltsersparnis völlig ausschließt, wie das oben referierte Ergebnis einer "nutzenorientierten Betrachtungsweise" zeigt.

\section{(2) Berücksichtigung häuslicher Dienste}

Selbsterstellte häusliche Dienstleistungen sind Teil der Haushaltsproduktion, die insgesamt wegen der damit verbundenen Bewertungs- und Ermittlungsproblematik sowie der Nähe zu Freizeitaktivitäten nicht erfaßt wird. In gewissem Umfang werden sie von allen Steuerpflichtigen bzw. in allen Haushalten erbracht, so daß einiges dafür spricht, sie wegen dieser

46 Vgl. Wissenschaftlicher Beirat beim BMF $(1967,351)$. Verfassungsrechtlich unbedenklich wäre wohl eine Option auf getrennte Veranlagung. Damit würden jedoch in einzelnen Fällen Abweichungen von der Norm der gemeinsamen Veranlagung zugelassen, was den Gleichbehandlungsgrundsatz zwar nicht in enger juristischer Auslegung, jedoch in seinem ökonomischen Gehalt verletzt. So auch Pohmer $(1968,153)$. 
für alle Steuerpflichtigen etwa gleichen Auswirkungen - zumal bei einer aus Praktikabilitätsgründen vorzunehmenden Pauschalierung - einkommensteuerlich außer acht zu lassen (Hackmann, 1983, 687).

Anlaß für eine Berücksichtigung der häuslichen Dienste gerade bei der Diskussion um die Ehegattenbesteuerung war die früher vorherrschende "Hausfrauenehe". Es war eine Arbeitsteilung der Ehepartner üblich, die einerseits dem Ehemann die Versorgung mit häuslichen Diensten ohne zusätzliche Beanspruchung seiner Freizeit gestattete, andererseits die Ehefrau von der Notwendigkeit einer Erwerbstätigkeit enthob. Die rechtliche Absicherung dieser Arbeitsteilung bot und bietet das Eherecht 47 , das gleichzeitig die Vermutung häuslicher Dienste durch einen nichterwerbstätigen Ehegatten objektivierbar macht (im Gegensatz $\mathrm{zu}$ anderen Haushaltsgemeinschaften, wo nur mit rechtlich nicht gestützten Annahmen gearbeitet werden könnte). Kritikpunkte sind also die bei gleichem Markteinkommen größere Freizeit und der (möglicherweise) größere Umfang an häuslichen Dienstleistungen bei Erstellung durch einen nichterwerbstätigen Ehepartner ${ }^{48}$.

Insbesondere Befürworter einer stärkeren Erwerbsbeteiligung von Frauen beklagen darüber hinaus die steuerliche Diskriminierung von Zweitverdienern aufgrund der Steuerfreiheit häuslicher Dienste. Aus Gründen der Steuerneutralität müßte die Haushaltstätigkeit mit ihren Opportunitätskosten bewertet werden ${ }^{49}$, aus "Schatteneinkommen" (vgl.

$47 \S 1360$ Satz 2 BGB besagt: "Ist einem Ehegatten die Haushaltsführung überlassen, so erfüllt er seine Verpflichtung, durch Arbeit zum Unterhalt der Familie beizutragen, in der Regel durch die Führung des Haushalts."

48 Bei Kanzler $(1988,211)$ findet sich die entgegengesetzte Aussage, häusliche Dienste stellten eine Minderung der steuerlichen Leistungsfähigkeit dar, was aber wohl im Vergleich zu einem potentiell möglichen Einkommen gemeint ist.

49 Bei Spahn $(1989,32)$ wird umgekehrt die steuerliche Bewertung der Erwerbstätigkeit mit dem "Hausfraueneinkommen" vorgeschlagen, was bei einer Subsumierung des "Hausfraueneinkommens" unter das steuerfreie Existenzminimum zur Steuerfreiheit der Zweitverdienereinkommen, in letzter Konsequenz (alle Erwerbseinkommen sind Substitute von Freizeit bzw. häuslicher Tätigkeit) sogar zur Steuerfreiheit aller Erwerbseinkommen führt. 
Kullmer, 1960, 89) müßte steuerliches Einkommen werden. Offensichtlich wäre dies jedoch nur innerhalb einer Soll-Einkommensteuer systemgerecht. Als Alternative innerhalb einer Ist-Einkommensteuer könnte an die Besteuerung der Haushaltsproduktion selbst gedacht werden (bei Bewertung zu den Kosten des Fremdbezugs), was die steuerliche Diskriminierung einer Erwerbstätigkeit allerdings nur mildern würde.

Für eine Umsetzung der vorgetragenen Kritik in die Steuerpraxis kommen zwei Wege in Frage: Der direkte Weg liegt in der Erhöhung der Bemessungsgrundlage um das Realeinkommen durch selbsterstellte häusliche Dienste, ein indirekter Weg steht mit der einkommensteuerlichen Anerkennung fremdbezogener Dienstleistungen in Form von Werbungskosten bzw. Betriebsausgaben zur Verfügung (objektivierbare Mehrausgaben bei Erwerbstätigkeit).

Wenn die Bemessungsgrundlage um den Wert der selbsterstellten häuslichen Dienste erhöht wird, muß gleichzeitig auch der Existenzminimum-Grundfreibetrag angehoben werden, da eine Grundversorgung mit häuslichen Diensten notwendig ist 50 . Wird im Rahmen einer notwendigen Pauschalierung davon ausgegangen, daß jeder Steuerpflichtige häusliche Dienste selbst erstellt, sofern er sie nicht durch Dritte erstellen läßt, was plausibel ist, dann wird durch die hier dargelegte Regelung ein Steuerfreibetrag im Ausmaß der erwähnten Erhöhung des Existenzminimum-Grundfreibetrags lediglich bei Fremdbezug von häuslichen Dienstleistungen eingeräumt (Fall 1). Die alternative Annahme, lediglich Nichterwerbstätige stellten häusliche Dienste zur Verfügung, ist offensichtlich weniger an der Realität orientiert. Dafür würden dann alle Erwerbstätigen, im Fall von Ehegatten alle Doppelverdiener, von einem Freibetrag profitieren 51 , was dem oben vorgetragenen Argument der eingeschränkten Freizeitmöglichkeiten Rechnung trägt (Fall 2).

Eine Anerkennung von Ausgaben für fremdbezogene häusliche Dienstleistungen als Werbungskosten bzw. Betriebsausgaben bei Erwerbstätigkeit würde in die gleiche Richtung wirken. Bei einer Anerken-

50 So auch Spahn $(1989,31)$.

51 So schlägt Pechman $(1966,89$ f.) einen Freibetrag in Höhe eines Prozentsatzes vom Erwerbseinkommen bis zu einer Höchstgrenze vor. 
nung tatsächlicher Ausgaben würden allerdings auch höhere Beträge abzugsfähig52 (Fall 3), die Anerkennung einer Pauschale für alle erwerbstätigen Alleinstehenden bzw. Doppelverdiener-Ehegatten käme wiederum einem Erwerbstätigenfreibetrag bzw. dessen Erhöhung gleich und zielte indirekt auf eine Besteuerung der Freizeit (Fall 4).

Bei einem Teil der skizzierten Lösungsansätze (Fälle 1 und 3) würden diejenigen Erwerbstätigen, die gleichwohl noch häusliche Dienste unter verstärktem Einsatz ihrer Freizeit selbst erbringen, benachteiligt. Im Gegensatz zum Regelfall der Werbungskosten bzw. Betriebsausgaben, bei dem spezielle Belastungen eines Teils der Steuerpflichtigen berücksichtigt werden, liegt hier der Fall vor, daß alle Erwerbstätigen von einer Belastung getroffen werden. Die bestehende Belastung wird lediglich unterschiedlich aufgefangen (durch selbsterstellte oder fremdbezogene Leistungen), nicht zuletzt auch eine Folge der jeweiligen finanziellen Möglichkeiten. Eine Berücksichtigung lediglich des Fremdbezugs häuslicher Dienstleistungen (durch einen Freibetrag oder in Höhe tatsächlicher Aufwendungen) begünstigt hier faktisch die Bezieher hoher Einkommen und ist daher abzulehnen 53. Unter Gerechtigkeitsaspekten ist die Berücksichtigung eines Pauschbetrags bei allen Erwerbstätigen vorzuziehen (Fälle 2 und 4). Eine solche Regelung zielte allerdings indirekt auf eine Besteuerung der Freizeit, ohne die tatsächliche zeitliche Belastung durch Erwerbstätigkeit in Rechnung stellen zu können (eine Differenzierung zwischen Vollzeit- und Teilzeitbeschäftigungen wäre aus Praktikabilitätsgründen schon bei abhängig Beschäftigten kaum möglich und bei Selbständigen noch aufwendiger) 54 .

52 Gegebenenfalls zu beschränken auf die Höhe des Erwerbseinkommens bzw. bei Ehegatten auf die Höhe des niedrigeren Erwerbseinkommens. Auch besteht die Möglichkeit einer allgemeinen Obergrenze, um Luxusaufwendungen auszuschließen.

53 Auch Tipke/Lang $(1991,379)$ sind über die ausnahmsweise Anerkennung von Unterhaltsaufwendungen der gehobenenen Lebensführung im Rahmen des § 10(1) Nr.8 EStG ("Dienstmädchenprivileg") erstaunt.

54 Es stellte sich auch die Frage, wie mit Nichterwerbstätigen umgegangen werden sollte, deren zeitliche Belastung ohne die sog. häuslichen Dienste bereits einer Vollzeitbeschäftigung entspricht: z.B. bei häuslicher Pflege, Kinderbetreuung, aber auch bei Studenten! 
Die Berücksichtigung eines Sonderfreibetrags bei alleinstehenden Erwerbstätigen sowie Doppelverdiener-Ehepaaren (je Person unter Berücksichtigung einer Haushaltsersparnis auch bei den häuslichen Diensten) scheint daher praktikabel und vertretbar (Andel, 1980, 384; Haller, 1981b, 22; Lang, 1983, 116), es bleiben aber einige Zweifel. Meines Erachtens überwiegen die Argumente für eine einkommensteuerliche Nichtberücksichtigung häuslicher Dienste, jedenfalls in dieser allgemeinen, alle Haushalte betreffenden Form. Dafür spricht auch, daß die Diskussion insbesondere durch die "Hausfrauenehe" ausgelöst wurde, deren Begünstigung zunächst in anderen, gewichtigeren Bereichen (Sozialversicherung) aufgehoben werden könnte und deren Verbreitung bereits aus anderen Gründen stark rückläufig ist.

\section{(3) Änderungen im Lohnsteuerabzugsverfahren zur Gleichstellung von Erst- und Zweitverdiener}

Obwohl, wie oben bereits ausführlich dargelegt wurde, jede Berücksichtigung von Unterhaltsverpflichtungen dazu führt, daß bei einer marginalen Betrachtung das Einkommen von "Zweitverdienern" höheren Steuersätzen als dasjenige vergleichbarer Alleinstehender unterworfen wird, besteht die Möglichkeit, auf das sozialpsychologische und damit auch auf das innereheliche Klima einzuwirken und damit einer Verfestigung der Positionen von Erstverdiener (i.d.R. Ehemann) und Zweitverdiener (i.d.R. Ehefrau) entgegenzusteuern. Prägend für den Eindruck der meisten Steuerzahler ist weniger eine ökonomische Analyse, wie sie oben angedeutet wurde, als vielmehr das Lohnsteuerabzugsverfahren, dessen Ergebnis direkt der monatlichen Gehaltsabrechnung zu entnehmen ist. Unterschiedliche Steuerklassen (bei Wahl der Kombination III und V) führen hier zu dem Eindruck, der "Zweitverdiener" (Klasse V), insb. auch die "Zweitverdienerin", trage wegen der höheren (durchschnittlichen) Steuerbelastung in geringerem Umfang als es dem Verhältnis der Bruttoeinkommen entspricht zum Gesamteinkommen bei. Die persönliche Leistung von Zweitverdienern mit Steuerklasse V wird damit (zumindest dem Anschein nach) diskriminiert und ihre Verhandlungsposition im Innenverhältnis der Ehepartner geschwächt. Diese Bedenken können auch 
nicht mit dem Hinweis auf die freiwillige Wahl der Steuerklassen III und V (statt IV und IV) unter Zustimmung beider Ehepartner ausgeräumt werden, da sich "Zweitverdiener" in einem Dilemma befinden: Einerseits kann es im Hinblick auf die Besteuerung des Gesamteinkommens rational sein, die Steuerklassenkombination III/V zu beantragen (allerdings nur wegen des damit verbundenen Zinsgewinns, was längst nicht allen Steuerpflichtigen klar sein dürfte), andererseits wird der eigene Beitrag zum Gesamteinkommen dadurch verzerrt wiedergegeben, ohne daß auch nur eine ungefähre Vorstellung von der Größenordnung dieser Verzerrung vorliegt. Daher sollte die Wahlmöglichkeit unterschiedlicher Steuerklassen abgeschafft werden, was sich wohl auch verfassungsrechtlich mit dem Gleichbehandlungsgebot (Art. 3 GG) rechtfertigen läßt, möglicherweise sogar aus verfassungsrechtlicher Sicht geboten ist. Dann wäre die Steuerbelastung durch Lohnsteuer für beide Verdiener nur noch vom individuellen Einkommen abhängig. Eine Zusammenführung der individuellen Einkommen in Form der Berücksichtigung innerehelicher Transfers fände dann im Regelfall erst im Lohnsteuerjahresausgleich bzw. in einer Einkommensteuerveranlagung statt. Damit würde die Berücksichtigung des Tatbestandes "Ehe" transparenter, weil auf einer Stufe des Besteuerungsverfahrens nachvollziehbar. Steuervereinfachend für den Steuerzahler wirkte eine mögliche Reduzierung der Zahl der Steuerklassen auf zwei, eine für die erste, eine für weitere Lohnsteuerkarten (wobei dann aber in einer größeren Zahl von Fällen weitere Freibeträge einzutragen wären).

Nachteilig ist allein, daß sich in einer größeren Zahl von Fällen als heute eine Steuerüberzahlung im Lohnsteuerverfahren ergibt. Diese kann selbstverständlich im Lohnsteuerjahresausgleich bzw. in der Einkommensteuerveranlagung geltend gemacht werden. Es bleiben allerdings ein Liquiditätseffekt und ein Zinseffekt zuungunsten der Steuerpflichtigen bestehen. Durch den Eintrag eines Freibetrages für den nicht erwerbstätigen Ehegatten oder, was wegen der persönlichen Zuordnung Vorteile hat, durch "Belastung" der Lohnsteuerkarten nicht erwerbstätiger Ehegatten in Höhe des Grundfreibetrages könnte dieser Effekt weitgehend vermieden werden. Auch könnte eine Verzinsung von Steuernachforde- 
rungen und -erstattungen nach $\S 233 \mathrm{a}$ AO bereits zur Mitte des Steuerentstehungsjahrs vorgenommen werden.

\subsubsection{Sonderfälle}

\section{(1) Zur Behandlung nichtehelicher Lebensgemeinschaften}

Leitidee der Behandlung nichtehelicher Lebensgemeinschaften sollte nicht das Ziel einer generellen Angleichung an die rechtliche Situation der Ehe sein, was wohl auch von den Betroffenen so nicht gewünscht wird und gleichzeitig eine sehr restriktive Abgrenzung der "eheähnlichen Lebensgemeinschaft" mit sich bringen würde, sondern vielmehr eine sachgerechte Lösung für die einzelnen betroffenen Bereiche wie z.B. das Steuerrecht und das Unterhaltsrecht 55 . Bei der Überprüfung einzelner Sachgebiete ist dann jeweils festzustellen, ob und in welcher Form den Besonderheiten nichtehelicher Lebensgemeinschaften Rechnung getragen werden kann 56 .

Durch die vorgeschlagene Form der Ehegattenbesteuerung findet keine Diskriminierung anderer Lebensformen statt, da ausdrücklich auf die jeweilige rechtliche Ausgangssituation Bezug genommen wird, die offensichtlich - auch in wirtschaftlicher Hinsicht - unterschiedlich ist. In nichtehelichen Lebensgemeinschaften bestehen keine rechtlichen Ansprüche auf Einkommenstransfers, d.h. die jeweiligen individuellen (Erwerbs-)Einkommen geben gleichzeitig die individuellen ökonomischen Verfügungsmöglichkeiten richtig wieder. Allerdings entspricht es den oben 57 aufgestellten Forderungen, Unterhaltszahlungen zur Sicherstellung des Existenzminimums des Empfängers ungeachtet seiner persönlichen Stellung zum Leistenden steuerlich anzuerkennen (Abzug von der Bemessungsgrundlage beim Leistenden, Hinzurechnung beim Empfänger),

55 Im Ansatz ähnlich auch D.Schwab (1991, 343-349).

56 Im Rahmen einer solchen Bestandsaufnahme können auch Erkenntnisse darüber erwartet werden, ob die jeweils für die Ehe getroffenen Regelungen noch zeitgemäß sind.

57 Vgl. Kapitel III.3.2. 
zumal ein Mißbrauch praktisch ausgeschlossen ist 58 . Eine steuerliche Anerkennung von darüber hinausgehenden freiwilligen Unterhaltsvereinbarungen kommt ohne weiteres nicht in Betracht, auch wenn eine schriftliche Abmachung vorliegen sollte, weil dann reine "Steuersparverträge" möglich sind, deren Gesamtkontext finanzamtlich nicht nachvollziehbar ist.

Daß nichteheliche Lebensgemeinschaften gegenüber ehelichen Lebensgemeinschaften nicht diskriminiert werden, zeigt auch der Vergleich mit Ehen, in denen Gütertrennung vereinbart ist, da diese nach den hier gemachten Vorschlägen wegen der nicht in allgemeiner Form abschätzbaren ökonomischen Beziehungen der Eheleute ebenso (oder zumindest ähnlich) behandelt werden sollten wie nichteheliche Lebensgemeinschaften. Denkbar wäre allerdings die steuerliche Anerkennung von klar klassifizierbaren und auf Dauer angelegten ökonomischen Bindungen zwischen Nicht-Eheleuten, wie sie in einer notariell beurkundeten und ggf. ins Güterrechtsregister einzutragenden Güter- bzw. Zugewinngemeinschaftsvereinbarung zum Ausdruck kommen könnte, da deren ökonomischer Gehalt mit dem einer entsprechenden ehelichen Vereinbarung vergleichbar wäre 59 .

Darüber hinaus ist eine Annäherung der steuerlichen Behandlung verschiedener Lebensformen dann angebracht, wenn die einkommensteuerliche Beeinflussung der vorhandenen Präferenzen über den verfassungsrechtlich gebotenen Schutz von Ehe und Familie hinausgeht. Ein wichtiger Schritt ist hier die steuerliche Anerkennung von Transfers zur Sicherung des Existenzminimums. Auch eine Anpassung des ehelichen Güterrechts an die Lebenswirklichkeit der Ehe (Modell einer familienphasenspezifischen Regelung) wirkt in diese Richtung.

Als Vorteil mag der Umstand angesehen werden, daß mögliche laufende Transfers im Rahmen einer gemeinsamen Lebensführung ein-

58 Allenfalls könnte noch eine Restriktion in bezug auf mögliches Vermögen des Empfängers sinnvoll sein.

59 Mögliche Adressaten einer solchen Regelung könnten auf Dauer angelegte Lebensgemeinschaften sein, die gleichwohl nicht als Ehe klassifiziert werden wollen oder können. 
kommensteuerlich nicht erfaßt werden (wie auch innerfamiliäre Leistungen und andere private Transaktionen, soweit sie nicht einen gewissen Umfang überschreiten oder über Marktvorgänge abgewickelt werden) 60 . Dies mag unter steuersystematischen Gesichtspunkten zwar als unbefriedigend betrachtet werden, führt jedoch dazu, daß ein möglicher "Steuernachteil" nichtehelicher Lebensgemeinschaften auf die Tarifprogression beschränkt bleibt und im angesprochenen Bereich gemeinsamer Einkommensverwendung nicht durch eine Erfassung von Transfers verstärkt wird.

Die Erfassung der Vorteile gemeinsamer Haushaltsführung ist im Hinblick auf eine mögliche Diskriminierung ehelicher oder nichtehelicher Lebensgemeinschaften unproblematisch, da sich beide Lebensformen hier gleich behandeln lassen. Allerdings sind häusliche Dienste innerhalb nichtehelicher Lebensgemeinschaften nicht pauschal unterstellbar, was jedoch bei einer generellen Nichtberücksichtigung häuslicher Dienste ohne Folgen bleibt sowie auch bei einer Berücksichtigung durch Freibeträge keine wesentliche Veränderung in der rein steuerlichen Vorteilhaftigkeit verschiedener Lebensgemeinschaften bewirken dürfte.

\section{(2) Zur Problematik von Änderungen in Lebensgemeinschaften}

Grundsätzliche konzeptionelle Probleme treten bei Änderungen von Lebensgemeinschaften dann auf, wenn die Einkommensbesteuerung nicht auf Individuen, sondern auf verschiedene Typen von Haushalten ausgerichtet ist, deren individuelle Zusammensetzung variiert61. Bei dem in dieser Arbeit verfolgten Konzept einer individualbezogenen Besteuerung wird diese Problematik weitestgehend vermieden, indem zunächst die jeweiligen individuellen Steuerbemessungsgrundlagen unter Berücksichtigung individualbezogener Freibeträge ermittelt werden, die sodann nach

60 Ebenso werden von der Norm abweichende innereheliche Transfers im Rahmen der gemeinsamen Lebensführung nicht erfaßt.

61 Vgl. Kapitel VI.2. 
einer Art "Baukastensystem" um haushaltsbezogene Freibeträge ergänzt werden (können) 62 .

Auch nach einer Entscheidung für eine individualbezogene Grundkonzeption treten Probleme dann auf, wenn bedingt durch den Wechsel in Lebensgemeinschaften die personelle Zuordnung von Vermögen verändert wird oder eine Änderung in den laufenden Transferbeziehungen der beteiligten Personen erfolgt, die sowohl den Umfang als auch den Charakter von Transferleistungen betreffen kann. Die Problematik einer Änderung der Zurechnung von Vermögen läßt sich offensichtlich immer dann vermeiden, wenn - jedenfalls für einkommensteuerliche Zwecke eine personenbezogene Zuordnung vorgenommen wird, die auch bei Änderungen in Lebensgemeinschaften nicht revisionsbedürftig wird. Sofern laufende Transferleistungen vorliegen, muß deren einkommensteuerliche Behandlung sowohl in bestehenden Lebensgemeinschaften als auch nach deren Auflösung geregelt werden; einer Aufrechterhaltung von Transferbeziehungen - i.d.R. für eine Übergangszeit - auch nach Auflösung von Lebensgemeinschaften wird dabei eine einkommensteuerliche Regelung am ehesten gerecht, die ebenfalls keine abrupten Wechsel vornimmt, sondern dem zivilrechtlich beabsichtigten allmählichen Übergang Rechnung trägt. Eine nähere Erörterung einkommensteuerlicher Probleme kann sich dabei auf rechtlich typisierte Lebensgemeinschaften, also vor allem diejenigen von Eltern und Kindern und von Ehegatten, beschränken.

Bei einer Änderung in Lebensgemeinschaften von Eltern und Kindern kann die einkommensteuerliche Behandlung von Transferleistungen an Kinder grundsätzlich beibehalten werden, allerdings führt ein eigener Hausstand von Kindern aufgrund des gestiegenen Bedarfs i.d.R. zu einer Erhöhung der Transferleistungen63, auch sollte ggf. eine vereinfachte

62 Damit ist allerdings nicht gemeint, daß eine beliebige Zuordnung individueller Steuerbemessungsgrundlagen ("interpersonales Korrespondenzprinzip") vorgenommen werden kann.

$63 \mathrm{Vgl}$. beispielsweise die Regelungen in $\S \S 12,13$ BAföG sowie $\S 33 \mathrm{a}(2)$ EStG. 
Veranlagung von Kindern in der Steuererklärung der Eltern dann entfallen. Vermögensrechtliche Probleme treten hier nicht auf.

Zwischen Ehegatten wird für einkommensteuerliche Zwecke bei Anwendung des Splittingverfahrens eine hälftige Aufteilung des Einkommens impliziert. Bei Auflösung der Ehe (nicht etwa bereits bei einem Getrenntleben der Ehegatten) entfällt nach der hier vertretenen Auffassung die rechtliche Grundlage hierfür. Treten gerichtlich festgesetzte Unterhaltszahlungen an die Stelle des Einkommenssplitting, dann sind diese jedoch wie innereheliche Transfers als zwangsläufige Folge der Eheschließung zu betrachten und damit beim Transferleistenden leistungsfähigkeitsmindernd und beim Transferempfänger leistungsfähigkeitserhöhend zu berücksichtigen. Ihr Umfang wird regelmäßig unterhalb der Zahlungen liegen, die bei bestehender Ehe zu berücksichtigen sind. Wird den Vorschlägen zur einkommensteuerlichen Behandlung von Ehen mit vereinbarter Gütertrennung gefolgt 64 , nach denen ein Einkommenssplitting eng begrenzt wird, könnte die Situation eintreten, daß gerichtlich festgesetzte Unterhaltszahlungen zwischen Getrenntlebenden oder Geschiedenen den bei bestehender Ehe angenommenen Transferumfang überschreiten. Eine einkommensteuerliche Anerkennung der vollen Unterhaltsaufwendungen ist auch in diesem Fall angebracht, da die gerichtliche Festsetzung von Unterhaltsleistungen deren Umfang objektiviert und die Verfügungsmacht über als Geldzahlung zu leistenden Unterhalt (§ 1585(1) BGB) offensichtlich voll beim Empfänger liegt.

Vermögensrechtliche Auseinandersetzungen im Scheidungsfall betreffen den Ausgleich von Vermögensrechten i.e.S. (Zugewinnausgleich; D.Schwab, 1991, 109-130) und den Ausgleich von Versorgungsanwartschaften (Versorgungsausgleich; D.Schwab, 1991, 179-190). Von entscheidender Bedeutung ist hier der vereinbarte Güterstand. Bei Gütergemeinschaft wird das Gesamtvermögen hälftig zwischen den Ehepart-

64 Vgl. Kapitel VI.3.2.3.2.(3). 
nern aufgeteilt, ebenfalls bei der Zugewinngemeinschaft der während der Ehe erzielte Vermögenszuwachs. Beiden Güterständen wird im Rahmen der Einkommensteuer das Ehegattensplitting gerecht, das bereits während der Ehe eine entsprechende Zuteilung des Vermögenszuwachses vornimmt bzw. vorwegnimmt 65 . Gütertrennung schließt jedenfalls in reiner Form Vermögensansprüche bei Ehescheidung aus; werden aufgrund von Modifikationen der Gütertrennung Vermögensübertragungen gerichtlich festgesetzt, so ist dieser Vorgang als einkommensteuerlich unerheblich zu betrachten, weil und sofern 66 er rückwirkend die personelle Zuordnung während der Ehe erzielten Einkommens verändert. Es sollte jedoch lediglich der Transferbetrag steuerfrei bleiben; eine entsprechende nachträgliche Korrektur der Steuerveranlagungen für die Zeit der Ehe ist aus Gründen der Transparenz und Praktikabilität wenig erstrebenswert.

Prinzipiell können die gleichen Grundgedanken auf den Versorgungsausgleich übertragen werden, so daß Übertragungen von Ansprüchen im Rahmen des Versorgungsausgleichs in der Regel einkommensteuerfrei bleiben können. Gerade im Bereich des Versorgungsausgleichs treten aber indirekt steuerliche Probleme auf, wenn die einkommensteuerliche Behandlung der verschiedenen Vorsorgeaufwendungen und Versorgungsleistungen völlig unterschiedlich ist, da deren Vergleichbarkeit dadurch stark beeinträchtigt wird. Hinzu kommt, daß ein Splitting von Ansprüchen aus institutionellen Gründen nicht ohne weiteres möglich

65 Im Falle der Gütergemeinschaft ist allerdings zum Zeitpunkt der Eheschließung ein Transfer zwischen den Ehegatten steuerlich zu erfassen, da hier zu diesem Zeitpunkt die persönliche Zuordnung von Vermögenswerten verändert wird.

66 Eine Ausnahme bildet die Abfindung von Unterhaltsansprüchen durch einen Kapitalbetrag, die jedoch als solche auszuweisen ist (§ 1585(2) BGB; Borth, 1989, 830-832). 
ist 67 , so daß auch die Aufteilung jeder einzelnen Anspruchsposition als Möglichkeit ausscheidet. Schließlich wird die Aufteilungsmasse selbst verändert, wenn bei Scheidung sonst vorhandene Ansprüche auf Hinterbliebenenversorgung ohne Ausgleich (Ausnahme: Erziehungsrenten nach $\S 47$ SGB VI) entfallen68. Voraussetzung einer insgesamt befriedigenden Behandlung des Versorgungsausgleichs ist daher neben einer Vereinheitlichung der einkommensteuerlichen Behandlung der Altersversorgung die Reform der Alterssicherungssysteme hin zu einer vorhersehbaren und vor allem beitragsbezogenen Leistungsgewährung, die eine personelle Zuordnung von Ansprüchen bereits während der Ehe ermöglicht (Splitting analog zur Einkommensteuer) ${ }^{69}$.

\section{Allgemeiner Kinderlastenausgleich}

Belastungen in Form von Unterhaltsaufwendungen für Kinder ${ }^{70}$ stellen keine Aufwendungen dar, die anderen Einkommensverwendungen ver-

67 Aus einer Vielzahl von Regelungen sollen hier nur einige Beispiele genannt werden: Nichtlinearitäten in der Bewertung von Anspruchsjahren (z.B. Beamtenversorgung), Festlegung der Bewertung von Anspruchszeiten erst bei Renteneintritt (z.B. Ausfallzeiten-Bewertung in der gesetzlichen Rentenversicherung, Orientierung am letzten Gehalt in der Beamtenversorgung), Verfall von Ansprüchen (z.B. Wartezeit-Regelung in der gesetzlichen Rentenversicherung, Ausschluß aus der Dynamisierung von Ansprüchen in der Zusatzversorgung im öffentlichen Dienst). Bezeichnenderweise wird in allen diesen Fällen gegen das Äquivalenzprinzip verstoßen; eine Orientierung am Äquivalenzprinzip bzw. bereits eine abschließende Bewertung zum Zeitpunkt der Entstehung von Anspruchszeiten würde daher auch die angesprochenen Probleme eines "Anspruchssplitting" beseitigen.

68 Für den Fall der Ehescheidung mögen die damit verbundenen Anreizwirkungen noch beabsichtigt sein, im umgekehrten Fall des Erwerbs von Ansprüchen auf volle Hinterbliebenensicherung bei Kurzzeit-Ehen wohl kaum; unter Gerechtigkeitsaspekten sind beide abzulehnen (so im Ansatz auch Ruland, 1973, 424, 426-428).

69 Vgl. hierzu, allerdings unter der Annahme einer Ertragsanteilsbesteuerung der Rentenzuflüsse, Pollak (1979, 274f.).

70 Im weiteren wird die Kinder-Definition des $\S 32 \mathrm{EStG}$ zugrundegelegt. Eine Ausweitung auf den Personenkreis des $§ 2$ BKGG wäre sinnvoll. 
gleichbar wären. Vielmehr stellen Unterhaltsaufwendungen für Kinder, auch wenn von einer freien Entscheidung der Eltern für Kinder ausgegangen werden kann, sowohl in verfassungsrechtlicher (Art. 1 und 6 GG) als auch in privatrechtlicher ( $\S 1601-1615$ BGB) Hinsicht besondere Aufwendungen dar, die einkommensteuerlich als leistungsfähigkeitsmindernd zu berücksichtigen sind (Bundesverfassungsgericht, 1991a, 87). Allerdings ist die Eltern-Kind-Beziehung in ihrem wirtschaftlichen Gehalt nicht als Erwerbsgemeinschaft, sondern lediglich als Unterhaltsbeziehung zu charakterisieren (Lang, 1983, 114).

Beachtet werden sollen hier nur solche staatliche Transfers und privatrechtliche Regelungen, die allen Kindern zugute kommen, ohne darüber hinaus besondere Anforderungen hinsichtlich der Bedarfssituation der Kinder oder der Einkommensverwendung bzw. des Beschäftigtenstatus der Eltern zu stellen 71 .

Auch ist hier nicht beabsichtigt, Diskussionen über die Vorteilhaftigkeit der Etablierung gesonderter Kinderlastenausgleichsfonds (Albers, 1967a; Zeppernick, 1974, 224-239) oder über die Verknüpfung von Kinderlastenausgleich und Altersversorgungssystem 72 aufzugreifen.

Anders als sonst innerhalb dieser Arbeit gewohnt, läßt sich die Analyse hier nicht auf die Frage der steuersystematisch richtigen Reaktion auf ein Bündel als gegeben zu betrachtender Transfers einschränken. Denn steuerliche Instrumente wie z.B. Kinderfreibeträge stehen aufgrund ihrer Zielsetzung, der Berücksichtigung der Leistungsfähigkeitsminderung von Eltern durch das Vorhandensein von unterhaltsberechtigten Kindern, in direkter Konkurrenz zum sozialpolitischen Instrument des Kindergeldes, da beide staatliche Maßnahmen die gleichen Anknüpfungspunkte benutzen und in der wissenschaftlichen und poli-

$71 \mathrm{Vgl}$. für die besondere Bedarfssituation der Ausbildung auch die Ausführungen in Kapitel IV.3. Umfassende Kataloge von kindbezogenen Regelungen des Steuer- und Sozialrechts finden sich z.B. bei Heldmann (1986, 75-80) sowie bei Schmidt/Frank/Müller-Rohr (1985, 34-42, 59-66). Die Zusammenstellung von Oberhauser $(1989,153-324)$ geht noch darüber hinaus.

72 Vgl. beispielsweise Schreiber (1955, 32-36), Nell-Breuning (1978), Schmidt/Frank/Müller-Rohr (1985, 45-50), Erbe (1986). 
tischen Diskussion eng miteinander verknüpft sind. Wie noch zu sehen sein wird, lassen sich zwar die rein steuersystematischen Zielsetzungen auch unabhängig von der Gestaltung des Kindergeldes verwirklichen, die sozialpolitischen Zielsetzungen des Kindergeldes erfordern jedoch eine Gesamtlösung unter Einbeziehung der steuerlichen Regelung. Daher wird hier eine abgestimmte Vorgehensweise von Steuer- und Sozialpolitik gefordert und das Kindergeld in die Analyse einbezogen 73 . Auf die konkreten Mängel einer jeweils isolierten Betrachtung von Steuer- und Sozialsystem wird später noch zurückzukommen sein.

\subsection{Anforderungen}

\subsubsection{Steuersystematische Anforderungen}

Leistungsfähigkeitsminderungen aufgrund von Kinderlasten ist aus steuersystematischer Sicht durch einen Abzug der Belastung von der Bemessungsgrundlage Rechnung zu tragen. Erst dann kann im Vergleich Steuerpflichtiger mit Kindern zu kinderlosen Steuerpflichtigen horizontale Steuergerechtigkeit als verwirklicht gelten 74 . Voraussetzung hierfür ist, daß Kinder nicht als reine Privatangelegenheit betrachtet werden, die Aufwendungen von Eltern für ihre Kinder nicht auf eine Stufe mit beliebigen Konsumentscheidungen gestellt werden (Bundesverfassungsgericht, 1991a, 87). Dafür spricht alleine schon die Achtung der Menschenwürde der Kinder, die auch in der zivilrechtlichen Zuerkennung eigenständiger Ansprüche gegenüber den Eltern zum Ausdruck kommt. Hinzu kommt

$73 \mathrm{Vgl}$. aus den zahlreichen finanzwissenschaftlichen und sozialpolitischen Arbeiten, die von einer Funktionsgleichheit von Kindergeld und Kinderfreibeträgen ausgehen z.B. Wissenschaftlicher Beirat beim BMF (1971, 525-528) und Oberhauser (1985); siehe auch Bundesverfassungsgericht (1991a, 83f.). Dagegen plädieren für eine strikte Trennung von Steuerund Sozialrecht, allerdings bei Anrechnung sozialpolitisch motivierter Entlastungen auf Freibeträge des Steuerrechts, Bachmann (1982, 320f.), Söhn $(1988,158 f$.) und Lang $(1990,339 f$.).

74 Vgl. z.B. Wissenschaftlicher Beirat beim BMF (1971, 522f.), Steuerreformkommission (1971, 203, Rz.611), Bachmann (1982, 319). 
der gesamtgesellschaftliche Aspekt der Bedeutung von Kindern für die Zukunft einer Gesellschaft im allgemeinen, für die Altersversorgung der Vorgänger-Generation im besonderen.

Wenn damit die Frage der einkommensteuerlichen Berücksichtigung von Kindern bei ihren Eltern 75 bejaht werden kann, bleibt doch die Höhe der steuerlich zu berücksichtigenden Aufwendungen noch unbestimmt.

Im allgemeinen steigen sicherlich die kindesbedingten Aufwendungen mit steigendem Einkommen der Eltern ${ }^{76}$. Wird man diese Aufwendungen oberhalb eines minimalen Aufwands in Höhe des Existenzminimums wegen der effektiven Entscheidungsgewalt und der berücksichtigten Präferenzen auch zunehmend als Konsum der Eltern einstufen können, so ist doch nicht zu verkennen, daß auch das in Konfliktfällen maßgebliche Zivilrecht mit dem Elterneinkommen - jedenfalls bis zu einer Obergrenze ansteigende Unterhaltsverpflichtungen vorsieht (Kalthoener/Büttner, 1989, 3-5, 98-103; Köhler, 1987, 15-26). Zwar kann die Steuergesetzgebung auf Pauschalierungen zurückgreifen, ob sie dabei jedoch die aus dem Bürgerlichen Gesetzbuch ableitbaren mit dem Elterneinkommen ansteigenden Unterhaltsverpflichtungen außer acht lassen darf, ist umstritten 77 .

Andererseits wird ein Kinderfreibetrag als Grundfreibetrag für Kinder betrachtet, so auch vom Bundesverfassungsgericht, wenn es vom Familienexistenzminimum, das steuerfrei bleiben müsse, spricht 78 . Wenn der

75 Wozu hier alle Personen gerechnet werden, denen nach § 32 EStG ein Kinderfreibetrag zusteht.

76 So z.B. Haller (1973, 508f.), Steuerreformkommission (1971, 203, Rz.608), Wissenschaftlicher Beirat beim BMF (1971, 523).

77 So plädiert Lang $(1983,124)$ für eine Orientierung an den bürgerlichrechtlichen Regelungen, Tipke (1984, 128) hält eine solche Lösung wegen der damit verbundenen Verteilungswirkungen bereits für nicht umsetzbar. Die dahingehende Interpretation des Bundesverfassungsgerichtsurteils vom 22.2.1984 (1984, insb. 224f.) durch Vogel $(1984,203)$ ist nicht gerechtfertigt, wie auch die neuerliche Entscheidung vom 29.5.1990 (Bundesverfassungsgericht, 1991a, 91) explizit zeigt.

78 Vgl. Bundesverfassungsgericht (1991a, 85-91). Den Verweis auf die Steuerfreiheit der Unterhaltsleistungen beim Kind halte ich dort allerdings nur bedingt für gelungen. 
Gedanke eines "klassenspezifischen Existenzminimums" nicht weiterverfolgt wird, wie er meines Wissens zuletzt von der kanadischen Royal Commission on Taxation (1966, Bd.3, 5-12) vertreten wurde, dann ist unter diesem Aspekt ein vom Elterneinkommen unabhängiger Freibetrag vorzusehen. Dies würde auch bei umfassender steuerlicher Erfassung von Transferzahlungen keine Steuerzahlungspflicht beim Empfänger auslösen 79 und einen wesentlichen Beitrag zur Steuertransparenz leisten. Ein solcher Freibetrag befindet sich dann allerdings nicht in Übereinstimmung mit den zivilrechtlichen Regelungen (Karl-Bräuer-Institut des Bundes der Steuerzahler, 1989, 50f.; Bundesverfassungsgericht, 1991a, 91).

Neben konstanten oder mit dem Elterneinkommen ansteigenden Freibeträgen wird auch eine Einbeziehung von Kindern in das Splitting ("Familiensplitting") vorgeschlagen (Wissenschaftlicher Beirat beim BMF, 1967, 346-353; Haller, 1973, 509f.), was bezüglich seiner Wirkungen einen Sonderfall von mit dem Elterneinkommen ansteigenden Kinderfreibeträgen darstellt. Die Einbeziehung von Kindern in das Splittingverfahren ist jedoch im Ansatz verfehlt, da hier das Eltern-KindVerhältnis als Erwerbsgemeinschaft statt als Unterhaltsgemeinschaft eingeschätzt wird. Mit dem Elterneinkommen abnehmende Splittingdivisoren (Wissenschaftlicher Beirat beim BMF, 1967, 352f.) versuchen diesem Einwand zwar partiell (meist nur auf die Ersparnis bezogen) Rechnung zu tragen, die Gesamtlösung würde damit jedoch extrem intransparent. Ein weiterer Einwand liegt in der fraglichen rechtlichen Zuordnung der Steuerschuld im Rahmen der hier notwendigen gemeinsamen Veranlagung der Eltern mit ihren unterhaltsberechtigten Kindern (Steuerreformkommission, 1971, 197f., Rz.580-589; Lang, 1983, 124f.). Zur Erzielung gleicher Wirkungen sind daher nach dem Elterneinkommen differenzierte Kinderfreibeträge vorzuziehen.

Wenn auch grundsätzliche Erwägungen für die Anerkennung vom Elterneinkommen unabhängiger Kinderbelastungen - ausgedrückt in Kinderfreibeträgen - sprechen, so führen doch die zivilrechtlichen Unterhaltsbestimmungen in ihrer derzeitigen Fassung zu mit dem Einkommen 
in Grenzen steigenden Freibeträgen. Ein steuerpolitisches Dilemma scheint unausweichlich.

Typisch für die vorgestellten steuerlichen Lösungsansätze sind, bedingt durch die Progression des Einkommensteuertarifs, mit dem Elterneinkommen ansteigende effektive Entlastungsbeträge, die Ausdruck des progressiven Steuertarifs sind. Zusätzlich verstärkt wird dieser Effekt, wenn von konstanten Freibeträgen zu mit dem Elterneinkommen in Grenzen ansteigenden Freibeträgen oder zu einem Familiensplitting übergegangen wird.

\subsubsection{Sozialpolitische Anforderungen}

Aus sozialpolitischer Sicht sollten staatliche Leistungen an die Eltern zum Ausgleich kindesbedingter Belastungen mit steigenden Elterneinkommen abnehmen, da dann die Eltern finanzielle Belastungen zunehmend selbst tragen können. Allenfalls wenn die Kinder als Adressaten staatlicher Transfers angesehen werden, lassen sich auch konstante, vom Elterneinkommen unabhängige Leistungen rechtfertigen 80 .

Bei Elterneinkommen auf oder unter Sozialhilfeniveau ist eine volle Übernahme der durch Kinder hervorgerufenen finanziellen Belastungen - ggf. im Rahmen der Sozialhilfe - notwendig. Dies ist aber auch bei darüber liegenden Elterneinkommen noch angebracht, um zu verhindern, daß die Eigenbelastung der Eltern zu sprunghaft ansteigt, z.B. von Null bei Elterneinkommen auf Sozialhilfeniveau auf die vollen Aufwendungen (wenn auch abzüglich der Entlastung durch Kindergeld und/oder Kinderfreibeträge) bei nur geringfügig höheren Elterneinkommen 81 . Hierdurch würden nicht nur extrem hohe Marginalbelastungen zusätzlichen Einkommens vermieden, sondern auch allgemein die Arbeitsanreize dadurch erhöht, daß Wohlstandssteigerungen aus eigener Arbeits-

80 Für eine ausführliche Darlegung sozialpolitischer Motive für kindbedingte Transfers vgl. z.B. Wissenschaftlicher Beirat beim BMF (1971, 514-516), Heldmann (1986, 69-75), Schmidt/Frank/Müller-Rohr (1985, 30-33).

81 So auch Kausemann (1983, 311), Schmidt/Frank/Müller-Rohr (1985, 52), Wissenschaftlicher Beirat beim BMF (1971, 516f.); anderer Ansicht Lang (1983, 122). 
leistung für einen größeren Teil von (arbeitsfähigen) Sozialhilfeempfängern erreichbar würden, die sog. Armutsfalle (Atkinson, 1989, 181) an Bedeutung verlöre.

Steuerpolitische Maßnahmen können aus sozialpolitischer Sicht nicht überzeugen, da eine Entlastung geringverdienender Eltern offensichtlich so nicht möglich ist. Auch stehen die mit steigenden Elterneinkommen ansteigenden effektiven Entlastungen aufgrund von Kinderfreibeträgen in Widerspruch zu der sozialpolitischen Forderung nach einer mit steigenden Einkommen sinkenden oder allenfalls konstanten effektiven Entlastung82.

\subsubsection{Differenzierung nach Alter und Ordnungszahl der Kinder?}

Eine Differenzierung nach dem Alter der Kinder erfolgt jedenfalls im Rahmen der für den Minimalaufwand für Kinder wichtige Anhaltspunkte liefernden Sozialhilfe. Dort werden die kindspezifischen Kosten nach vier Altersgruppen differenziert und liegen derzeit (Neufestsetzung für Hessen zum 1.7.199283) zwischen $255 \mathrm{DM}$ und $459 \mathrm{DM}$ (50 bzw. $90 \%$ des Eckregelsatzes für den Haushaltsvorstand ohne anteilige Kosten für Unterkunft und Heizung sowie für Neuanschaffungen von Kleidern und Hausrat; vgl. Schulte/Trenk-Hinterberger, 1986, 157f.). Auch der Kindesunterhalt nach der Düsseldorfer Tabelle ist nach dem Alter des Kindes gestaffelt, allerdings mit einer geringeren Bandbreite (Institut für Familienrecht, 1992). Schließlich steht eine solche Vorgehensweise auch in Übereinstimmung mit den Ergebnissen verschiedener Arten von Äquivalenzskalenanalysen (Klein, 1986, 87, 90f.). Daher ist es nahe-

82 Auge (1984, insb. 364-367) löst diesen Gegensatz nur scheinbar auf, wenn er die Bedingungen für von ihm so genannte Kinderfreibeträge so konstruiert, daß sie in Wirklichkeit mit dem Elterneinkommen abnehmende Kindergeldzahlungen darstellen.

83 Vgl. Staatsanzeiger für das Land Hessen, 1992, 1771. 
liegend, auch bei Kinderfreibeträgen bzw. beim Kindergeld eine derartige Differenzierung durchzuführen 84 .

Allerdings bedeutete dies eine Komplizierung des Verfahrens, da z.B. für Eintragungen auf der Lohnsteuerkarte das Alter im Prinzip berücksichtigungsfähiger Kinder bedeutsam würde. Dieser Verwaltungsaufwand wäre immer dann ungerechtfertigt, wenn die Rahmenbedingungen, wie insbesondere die Zuordnung der Kinder zu Eltern, die Einkommen von Eltern und ggf. Kindern bezüglich des Einflusses auf die Entlastungswirkung von Steuerfreibeträgen sowie die Anspruchsvoraussetzungen für Steuerfreibeträge, Kindergeld und Sozialhilfe konstant blieben, da sich dann bei Berücksichtigung von durchschnittlichen Aufwendungen Unterund Überzahlungen im Zeitablauf ohnehin ausglichen 85 .

Altersspezifische Regelungen allein im Sozialhilferecht, die wegen der dort notwendigen Berücksichtigung des aktuellen Bedarfs notwendig wären, bedeuteten jedoch wegen der Abweichungen vom sonstigen Sozial- und Steuerrecht Systeminkonsistenzen. Unter systematischen Gesichtspunkten ist eine Staffelung von kindesbedingten Aufwendungen nach Altersklassen daher vorzuziehen, konkrete Regelungen sollten allerdings im Hinblick auf Verwaltungskosten und Transparenz überprüft werden.

Für eine Differenzierung kindesbedingter Aufwendungen nach der Ordnungszahl der Kinder finden sich zwei Argumente: Mit steigender Ordnungszahl sei einerseits eine Kostendegression möglich, andererseits gehe die den Eltern zumutbare Eigenbelastung zurück.

Aus einkommensteuerlicher Sicht ("Reduktion steuerlicher Leistungsfähigkeit") kommt lediglich der erste Faktor in Frage (Wissenschaftlicher Beirat beim BMF, 1971, 525), das Ausmaß einer möglichen Kosten-

84 Vgl. Wissenschaftlicher Beirat beim BMF (1971, 518), Pohmer (1968, 171) und Albers (1967a, 203). Wegen der Einbeziehung von Betreuungskosten, die allerdings nicht allen Eltern entstehen, anderer Ansicht: Kanzler $(1988,214)$.

85 Vgl. Haller (1981b, 28), Dornbusch (1982, 547f.). Auch das Bundesverfassungsgericht $(1991 \mathrm{a}, 91)$ läßt sich in seinem Urteil vom 29.5.1990 so interpretieren. 
degression dürfte aber gering sein 86 ; eine Kostendegression wird nicht einmal in den Sozialhilfe-Regelsätzen berücksichtigt.

Aus sozialpolitischer Sicht kommt auch der zweite Faktor in Betracht; das Ausmaß einer (überproportionalen) Bedarfszunahme bei steigender Kinderzahl hängt dabei vom Umfang der vorher vorgenommenen Kürzung staatlicher Leistungen um eine "zumutbare Eigenbelastung" ab, ist damit von der durch Einkommen und Familiengröße beschriebenen konkreten Familiensituation abhängig. Da eine solche Vorgehensweise nicht zu der in dieser Arbeit verfolgten Integration steuerlicher und sozialpolitischer Lösungen paßt, wird sie hier nicht weiter verfolgt 87 .

Für die weitere Analyse wird vereinfachend von einem durchschnittlichen Betrag pro Kind ausgegangen, der ohne Probleme grundsätzlicher Art nach dem Alter der Kinder differenziert werden könnte; eine Differenzierung nach der Ordnungszahl der Kinder wird aus den genannten Gründen abgelehnt.

\subsection{Unzulänglichkeiten des derzeitigen dualen Systems}

Wenn die Regelungen im Steuer- und Sozialsystem jeweils für sich den oben genannten Anforderungen gemäß ausgestaltet werden, so ist das Ergebnis gleichwohl unbefriedigend. Das liegt daran, daß der kumulierte Gesamtbeitrag von Steuer- und Sozialsystem zu den Unterhaltsaufwendungen für Kinder nicht überzeugen kann, sowohl was die Entwicklung der Gesamtentlastung in Abhängigkeit vom Elterneinkommen als auch was die Stetigkeit des Entlastungsverlaufs betrifft.

Typisch für das sog. duale System aus Steuerfreibeträgen und Kindergeld ist eine in Abhängigkeit vom Elterneinkommen U-förmige Entwicklung des effektiven Entlastungsbetrags pro Kind, verursacht

$86 \mathrm{Vgl}$. den Überblick über vorliegende Untersuchungen bei Klein (1986, 7986); die tatsächlichen Ausgaben dürften dagegen wegen der bereits vorhandenen Belastungen bei zunehmender Kinderzahl zurückgehen. Gegenteiliger Ansicht ist die Steuerreformkommission (1971, 206, Rz.629), allerdings ohne plausible Gründe zu nennen.

87 Als Vertreter einer solchen Lösung sei jedoch beispielhaft auf Albers (1967a, 203; 1977, 943f.) verwiesen. 
durch die Dominanz des Kindergeldes und der Sozialhilfe bei niedrigen Einkommen und der effektiven Steuerentlastung durch Kinderfreibeträge bei hohen Einkommen 88 . Auch die Einführung einer Wahlmöglichkeit zwischen Kindergeld und Kinderfreibeträgen (Oberhauser, 1985, 18; 1989, 63; Zimmermann, 1989, 153) ändert die Sachlage nicht grundlegend (Heldmann, 1986, 306-308). Zu fordern ist daher eine Gestaltung, die zu einem systematischen Verlauf der effektiven Entlastungsbeträge in Abhängigkeit vom Einkommen führt, was allerdings die Verständigung auf eine einheitliche Zielsetzung des Kinderlastenausgleichs voraussetzt ${ }^{89}$.

Ein Abbau des Kindergeldes mit zunehmendem Einkommen kann zu grundsätzlich unerwünschten Sprüngen im Entlastungsverlauf sowie zu hohen Marginalbelastungen des Einkommens 90 führen. Schwer begründbare Änderungen in der Zusammensetzung der Gesamtentlastung aus steuerlich und sozialpolitisch begründeten Regelungen können bei kinderzahl- und kinderordnungszahlabhängigen Gestaltungen hinzutreten (Oberhauser, 1985; Scherf, 1992).

Während die Befürworter einer primär steuerlichen Lösung zum Kinderlastenausgleich mit dem dualen System noch recht zufrieden sein können, da ihre Bedingung "Abzug der Unterhaltsaufwendungen für Kinder von der Steuerbemessungsgrundlage" von der Höhe des Kindergeldes unabhängig ist, liegt die Ablehnung insbesondere eines in Abhängigkeit vom Einkommen U-förmigen Entlastungsverlaufs aus sozialpolitischer Perspektive auf der Hand.

88 Verstärkt wird ein solcher Verlauf noch durch eine einkommensabhängige Reduktion des Kindergeldes bei mittleren Einkommen.

89 Vgl. für eine ausführliche Darstellung und kritische Bewertung verschiedener "dualer Lösungsansätze" insb. Heldmann (1986, 306-313), die dann allerdings ihren eigenen Ergebnissen widersprechend selbst ein duales System vorschlägt $(1986,319)$ und lediglich durch eine anschließende Umdefinition des Kinderlastenausgleichs (1986, 319f.: "Diese steuerliche Regelung ... zählt nicht zu den Maßnahmen des Kinderlastenausgleichs ...") ein konsistentes Modell vorstellen kann.

90 Ebenfalls durch Sprünge im Verlauf, aber auch durch stetige Verläufe wie nach dem Vorschlag von Heldmann (1986, 322-330), der Marginalbelastungen von $80 \%$ impliziert. 
Ein ausgezeichnetes Anschauungsobjekt für die Mängel des dualen Systems ist der deutsche Kinderlastenausgleich. Nach § $32 \mathrm{EStG}$ wird ein Steuerfreibetrag in Höhe von 4104 DM pro Kind eingeräumt. Daneben wird ein Kindergeld von $70 \mathrm{DM}$ für das erste, $130 \mathrm{DM}$ für das zweite, 220 DM für das dritte und 240 DM für das vierte und jedes weitere Kind gezahlt. Dieses wird bei Überschreiten bestimmter Einkommensgrenzen der Eltern allmählich bis auf Sockelbeträge von $70 \mathrm{DM}$ für das zweite und 140 DM für jedes weitere Kind zurückgenommen. Für Geringverdienende, die die Kinderfreibeträge des Einkommensteuergesetzes nicht oder nicht voll nutzen können, wird das Kindergeld maximal um die Höhe der Steuerentlastungsbeträge von Steuerpflichtigen in der Eingangsproportionalzone des Einkommensteuertarifs, also um 65 DM, aufgestockt. Zu ergänzen ist die Betrachtung um die Sozialhilfeleistungen für Kinder. Diese liegen nach für das Jahr 1990 durchgeführten Berechnungen als Gesamtleistungen im Durchschnitt der Altersgruppen bei 450 DM monatlich (Karl-Bräuer-Institut des Bundes der Steuerzahler, 1991, 19).

\subsection{Integrationskonzepte}

Wenn die jeweils eigenständigen Begründungen für einen Kinderlastenausgleich aus steuersystematischer und aus sozialpolitischer Sicht akzeptiert werden, stellt sich die Frage, wie das steuerliche Instrumentarium (Kinderfreibetrag) und das sozialpolitische Instrumentarium (Kindergeld) miteinander verknüpft werden können, damit ein akzeptables Gesamtkonzept entsteht. Die beiden grundlegenden Ansätze hierzu zeichnen sich durch Übertragung der jeweils dominierenden Sichtweise auf das zusätzlich in die Betrachtung einzubeziehende Instrument aus: einerseits die Einbeziehung des Kindergeldes in die Steuerpflicht, andererseits die Berechnung der effektiven Steuerentlastung durch Kinderfreibeträge und deren Abgeltung durch Kindergeld. 


\subsubsection{Besteuerung des Kindergeldes}

Den Ausgangspunkt bildet hier die steuersystematische Sichtweise: Es wird ein Kinderfreibetrag gewährt, durch den die zwangsläufigen Aufwendungen für den Kindesunterhalt von der Steuerbemessungsgrundlage abgezogen werden können. Wird daneben ein Kindergeld gezahlt, so erhöht dies die steuerliche Leistungsfähigkeit des Empfängers, woraus eine Einbeziehung in die Steuerbemessungsgrundlage gefolgert wird.

Unter sonst gleichen Umständen führt die Besteuerung des Kindergeldes zu einer in bezug auf das Elterneinkommen degressiven Ausgestaltung des (Netto-)Kindergeldes, was insoweit auch sozialpolitischen Forderungen entspricht 91 .

Aus einkommensteuersystematischer Sicht ist eine solche Ceterisparibus-Betrachtung allerdings nicht angebracht. Denn Kinderfreibeträge sind in ihrer Höhe so zu bemessen, daß die - nach Berücksichtigung staatlicher Transfers noch verbleibenden - zwangsläufigen Belastungen der Eltern steuerfrei gelassen werden 92 . Sofern das Kindergeld steuerfrei gestellt ist, reduziert sich daher der sonst erforderliche Kinderfreibetrag um die Höhe des Kindergeldes, andernfalls - das Kindergeld ist steuerpflichtig - ist dieser in der ursprünglichen Höhe zu gewähren ${ }^{93}$. Bei Beachtung dieses konzeptionellen Zusammenhangs ergibt sich das erstaunliche Resultat, daß das Kindergeld immer steuerfrei bleibt, ob über

91 Vgl. zu Forderungen nach einer Besteuerung des Kindergeldes z.B. Oeter (1967, 207) sowie Tipke (1984, 128). Denkbar ist auch eine Besteuerung des Kindergeldes in einem Kinderlastenausgleichssystem ohne Kinderfreibeträge, dann handelt es sich allerdings im Grunde um eine rein sozialpolitische Lösung, die sich lediglich zur Realisierung des gewünschten Entlastungsverlaufs und aus Verwaltungsvereinfachungsgründen der Einkommensteuer bedient; vgl. hierzu Busch $(1975,165)$ sowie Leibfritz/Parsche $(1988,200,203)$.

92 Vgl. Lang $(1983,108,110 ; 1984,131 ; 1990,340)$ und Schmidt/ Frank/Müller-Rohr (1985, 34).

93 Ein solcher Zusammenhang zwischen Steuerpflicht des Kindergeldes und Höhe des Kinderfreibetrages wird bereits von Busch $(1975,166)$ angedeutet. 
eine Sonderregelung wie in $\S 3$ Ziff.24 EStG oder über eine Erhöhung der Kinderfreibeträge im gleichen Umfang.

Der dargestellte konzeptionelle Zusammenhang an sich - Kürzung von sonst vorzusehenden Kinderfreibeträgen um staatliche Kindergeldzahlungen - stellt jedoch bereits ein Reformkonzept dar, das aus steuersystematischer Sicht eine konsequente Zusammenführung von Kindergeld und Kinderfreibeträgen ermöglicht, auch wenn die in einem Teil der Literatur vorausgesetzte Minderung der effektiven Höhe des Kindergeldes durch dessen Einbezug in die Steuerpflicht, wie gerade dargelegt wurde, gerade bei einer konsequent steuersystematischen Ausgestaltung des Systems ausbleibt.

\subsubsection{Konzeption der effektiven Gesamtentlastung}

Unter Bezugnahme auf die oben genannten Anforderungen an einen Kinderlastenausgleich wird hier die Betonung der sozialpolitischen Perspektive deutlich. Bei der Berechnung der effektiven Gesamtentlastung wird die Frage gestellt, wie sich aufgrund der Maßnahmen des Kinderlastenausgleichs das verfügbare Einkommen der Eltern verändert. Im Gegensatz zur ersten Konzeption handelt es sich um eine Betrachtung nach Steuern, in die die Entlastungen aus Kinderfreibeträgen und Kindergeld gleichermaßen eingehen. In der Praxis wird von Anhängern dieser Konzeption allerdings aus systematischen und Verwaltungsvereinfachungsgründen der Weg über das Kindergeld allein gewählt 94 , da dieses im Gegensatz zu Kinderfreibeträgen direkt die Zielgröße, den effektiven Entlastungsbetrag, angibt 95 .

94 Ein typisches Beispiel für diese Vorgehensweise ist die Ersetzung des (vorherigen) dualen Systems des Kinderlastenausgleichs durch ein einheitliches Kindergeld im Jahr 1974. Vgl. Deutscher Bundestag (1974, Einl., 212-214). Zur Diskussion dieses Vorgehens vgl. das Urteil des Bundesverfassungsgerichts $(1977,108-125)$ vom 23.11.1976.

95 Unter der Voraussetzung, daß das Kindergeld steuerfrei gestellt wird. Sonst ist die effektive Entlastung eine Funktion des Steuertarifs, zeichnet sich allerdings auch dann durch einen in Abhängigkeit vom Einkommen stetigen Verlauf aus, der zudem als Folge der progressiven Besteuerung degressiv ist. 
Rein technisch errechnet sich die Gesamtentlastung als Summe aus dem (steuerfreien) Kindergeldbetrag und der Steuerersparnis aus dem Kinderfreibetrag (Kinderfreibetrag multipliziert mit dem persönlichen marginalen Steuersatz). Umgekehrt ist das Verfahren auch zur Umrechnung von Kindergeldzahlungen in fiktive Kinderfreibeträge verwendbar 96 .

\subsubsection{Bewertung und eigener Vorschlag}

Die grundlegenden Forderungen an den Kinderlastenausgieich aus steuerlicher und sozialpolitischer Sicht lassen sich folgendermaßen formulieren 97 :

- Einkommensteuerliche Berücksichtigung der zwangsläufigen Unterhaltsaufwendungen für Kinder.

- Vermeidung einer mit dem Elterneinkommen ansteigenden Gesamtentlastung 98 .

- Gewährung der vollen (minimalen) Unterhaltsaufwendungen für Geringverdienende bei ggf. allmählichem Abbau mit steigendem Elterneinkommen.

Da der schon als klassisch zu bezeichnende Grundkonflikt auf dem Gebiet des Kinderlastenausgleichs zwischen den beiden ersten Forderungen besteht und die dritte Forderung einen ergänzenden Charakter hat, soll diese zunächst zurückgestellt werden. Aus den oben vorgestellten Integrationskonzepten ergeben sich dann folgende Lösungsmöglichkeiten:

96 So Karl-Bräuer-Institut des Bundes der Steuerzahler $(1989,64-69)$ sowie das Bundesverfassungsgericht in seiner Entscheidung vom 29.5.1990 (1991a, 91-97); ablehnend hierzu Lang (1990, 339f.).

97 Ähnlich der Katalog bei Leibfritz/Parsche $\left(1988,52^{*}\right)$. Dort wird allerdings zusätzlich das fiskalische Ziel betont.

98 Damit wird nicht bestritten, daß es sich bei der mit steigendem Elterneinkommen ansteigenden Entlastungswirkung von Kinderfreibeträgen lediglich um einen "Reflex des progressiven Einkommensteuertarifs" handelt, die aus Sicht der Steuergerechtigkeit bei einem Belastungsvergleich gleich Leistungsfähiger völlig in Ordnung geht. Hier liegt aber der umfassendere Ansatz der Transfergerechtigkeit zugrunde. 
(1) Unter Beibehaltung einer explizit steuerrechtlichen Lösung:

Ein Kinderfreibetrag in Höhe der zwangsläufigen Unterhaltsaufwendungen führt in Verbindung mit Kindergeld dann nicht zu einem mit dem Elterneinkommen ansteigenden Entlastungseffekt, wenn (a) das einkommensunabhängige (steuerpflichtige) Kindergeld genau dem Kinderfreibetrag entspricht, so daß letztlich die Kindergeldzahlung effektiv wird, da sich Steuerpflicht und Steuerfreibetrag gerade neutralisieren, oder wenn (b) ein Abbau des Kindergeldes (nach Steuern) mit steigendem Elterneinkommen genau den Anstieg der Entlastungswirkung durch den Kinderfreibetrag kompensiert, so daß auch hier eine konstante (steuerfreie) Kindergeldzahlung realisiert wird. Beide Vorgehensweisen unterscheiden sich lediglich durch die Höhe der Kindergeldzahlung, die im Fall (a) dem Kinderfreibetrag entspricht, im Fall (b) dagegen nur der steuerlichen Entlastungswirkung aus Kinderfreibetrag und Spitzensteuersatz. Bei einer Differenzierung der Kinderfreibeträge nach dem Elterneinkommen wegen ansteigender Unterhaltsverpflichtungen steigen die notwendigen Kindergeldzahlungen in beiden Fällen entsprechend an.

(2) Bei Kompensation von steuerlichen Regelungen durch Kindergeld:

Ein allen Steuerpflichtigen zu gewährendes Kindergeld ist in einer Höhe festzulegen, die der Steuerentlastung aus dem bei einer steuerrechtlichen Lösung vorzusehenden Kinderfreibetrag und dem Spitzensteuersatz entspricht. Da hier direkt die effektive Entlastung als Zielgröße festgelegt werden kann, ist das Kindergeld steuerfrei zu lassen; zusätzliche Steuerfreibeträge werden nicht gewährt. Steigen die aus rein steuersystematischer Sicht zu gewährenden Kinderfreibeträge mit dem Elterneinkommen an, erhöht das die allen Kindern (bzw. deren Eltern) zu gewährenden Kindergeldzahlungen entsprechend.

Es wird deutlich, daß die unter (1) vorgestellten Lösungen lediglich formal an einer steuerrechtlichen Regelung durch Kinderfreibeträge festhalten, im Ergebnis aber auch hier eine Kindergeldlösung wie in (2) realisiert wird. Transparenz- und Verwaltungsvereinfachungsgründe sprechen insbesondere gegen (1b). (1a) könnte unter diesen Aspekten noch akzeptiert werden, erfordert jedoch einen erheblich höheren Mitteleinsatz und ist daher ebenfalls abzulehnen. 
Aus diesen Gründen halte ich ein Kindergeld, das einen ausreichenden Ausgleich für das Wegfallen von Kinderfreibeträgen bietet - ggf. auch bei Berücksichtigung von tatsächlichen zwangsläufigen Unterhaltsaufwendungen und eines marginalen Steuersatzes in Höhe des Spitzensteuersatzes -, für die angebrachte Lösung. Dieses Kindergeld ist dann steuerfrei zu lassen, da parallel dazu keine Kinderfreibeträge gewährt werden, der gesamte allgemeine Kinderlastenausgleich damit abgeglichen ist. Um der Kritik aus dem steuerrechtlichen Lager an einer Kindergeldlösung zu begegnen, ist im Einkommensteuergesetz explizit anstelle von Kinderfreibeträgen auf die Ausgleichsfunktion des Kindergeldes hinzuweisen 99 . Damit ist rechtlich einwandfrei klargestellt, daß das Kindergeld die Funktion der steuerlichen Berücksichtigung kindesbedingter Belastungen mit übernimmt und auch in seiner Höhe an dieser Forderung zu messen ist. Auch verfassungsrechtlich ist eine solche Lösung voll abgedeckt, selbst wenn scheinbar auf eine steuerliche Berücksichtigung der Kinderlasten verzichtet wird 100 . In der gegenteiligen Argumentation von Lang101 ist zwar die Wesensverschiedenheit von Kinderfreibetrag und Kindergeld (obligatorischer bzw. fakultativer Familienlastenausgleich) nachvollziehbar, nicht jedoch die daraus gefolgerte unbedingte Ablehnung einer "Vermengung der Kindergeld- und Kinderfreibetragsfunktion", wozu auch der in diesem Zusammenhang unzutreffende Verweis auf eine angebliche Benachteiligung von "finanziell schwächeren Schichten" nichts beiträgt.

Eine Berücksichtigung der dritten der oben genannten Forderungen, Gewährung des vollen (minimalen) Unterhaltsbetrages an Geringverdienende, läßt sich wie folgt umsetzen: Sollte das Existenzminimum von Kindern oberhalb des Betrages liegen, der sich aus dem Produkt von

99 Etwa folgendermaßen: "Die allgemeine Minderung der steuerlichen Leistungsfähigkeit von Eltern mit unterhaltsberechtigten Kindern wird durch ein Kindergeld mit ausgeglichen." Dies setzt auch eine Angleichung der Kinder-Definitionen von Bundeskindergeldgesetz und Einkommensteuergesetz voraus.

$100 \mathrm{Vgl}$. die Urteile des Bundesverfassungsgerichts vom 23.11.1976 (1977, 108) und vom 29.5.1990 (1991a, 60, 84, 89f.).

101 Vgl. Lang (1990, 339f., Zitate auf S. 339). 
Kinderfreibetrag und Spitzensteuersatz ergibt (Kindergeld nach (2) wie beschrieben), so ist bei geringen Elterneinkommen ein Betrag in Höhe des Existenzminimums auszuzahlen und dann mit steigendem Elterneinkommen allmählich auf den Betrag, der das Steuerfreibetragsäquivalent darstellt, zu reduzieren. Für diesen Fall würde auch die Steuerpflicht eines allgemeinen Kindergeldes in Höhe des vollen Existenzminimumbetrages in die gleiche Richtung wirken (Leibfritz/Parsche, 1988, 200f., 203f.), jedoch nur für einen Sonderfall zu den gleichen effektiven Entlastungen führen 102 .

Damit sind die Eckpunkte einer Kinderlastenausgleichslösung durch Kindergeld beschrieben:

- Kindergeld in Höhe des Existenzminimums von Kindern für Eltern mit geringem Einkommen (A). Das Existenzminimum von Kindern ist in Anlehnung an die Bestimmungen der Sozialhilfe zu ermitteln. Sollte sich für das Kindergeld nach (B) ein höherer Betrag ergeben, so ist dieser allen Kindergeldempfängern zu zahlen.

- Kindergeld in Höhe des Produktes aus maximalen zwangsläufigen Unterhaltsaufwendungen für Kinder 103 und Spitzensteuersatz der Einkommensteuer für Eltern mit hohem Einkommen (B). Die maximalen Unterhaltsaufwendungen werden dabei in Höhe des für die oberste Einkommensgruppe der Düsseldorfer Tabelle angegebenen Tabellenwerts pauschaliert.

In diesen Eckpunkten unterscheidet sich der hier vorgetragene Vorschlag von den vom ifo-Institut zur Wahl gestellten Alternativen (Leibfritz/ Parsche, 1988, VII*f., 196-207) sowie von zahlreichen Vorschlägen

102 Der Eintritt dieses Sonderfalls ist vom Steuertarifverlauf, von der Festlegung des Existenzminimums und der Höhe der maximal berücksichtigungsfähigen Kinderlasten im Referenzsystem abhängig.

103 Die Notwendigkeit einer Erörterung mit dem Elterneinkommen ansteigender Unterhaltsverpflichtungen wird auch in der Arbeit von Leibfritz/Parsche $(1988,152)$ genannt, aber später nicht weiter beachtet. 
zur Einführung eines allgemeinen Kindergeldes bei gleichzeitiger Abschaffung von Kinderfreibeträgen 104 .

Grafisch läßt sich der hieraus ableitbare Kindergeldverlauf in Abhängigkeit vom Elterneinkommen wie in Abbildung 2 gezeigt beschreiben.

Abbildung 2:

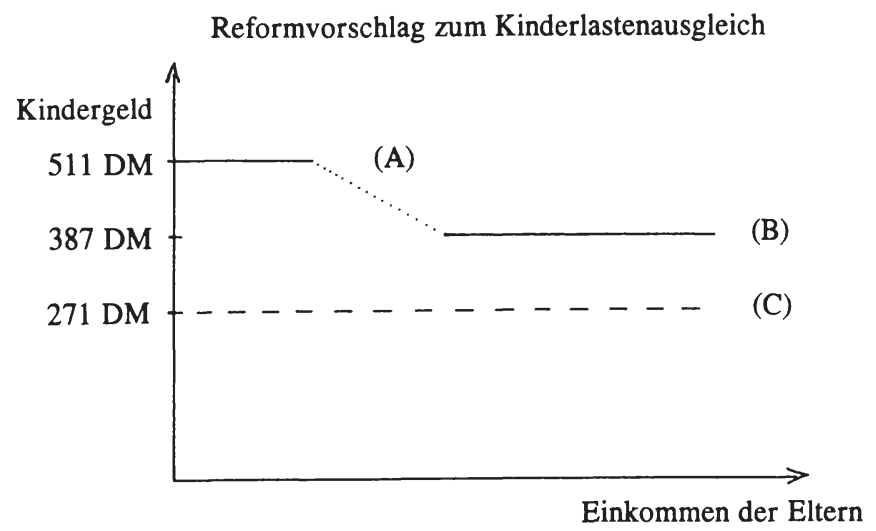

Quelle: Institut für Familienrecht (1992, 398); Karl-Bräuer-Institut des Bundes der Steuerzahler $(1991,19)$, fortgeschrieben mit der Entwicklung der Sozialhilfe-Regelsätze von 1990 bis 1992 (Staatsanzeiger für das Land Hessen, 1990, 1456; 1991, 1899; 1992, 1771); Angaben für das Jahr 1992.

Kindergeld wird bei niedrigen Elterneinkommen in Höhe des vollen Existenzminimums von Kindern (A) gezahlt, dann mit steigenden Elterneinkommen allmählich auf den Betrag (B) reduziert, der sich als Kompensation einer einkommensteuerlichen Berücksichtigung von Kinderlasten ergibt. Im mittleren Einkommensbereich (gepunktet dargestellter Verlauf) sollten die Grenzbelastungen des Einkommens erträglich bleiben; ein linearer Verlauf ist nicht unbedingt notwendig.

Ein Betrag (C) ergibt sich dann, wenn - der Argumentation des Bundesverfassungsgerichts (1991a, 91) folgend - davon ausgegangen wird,

$104 \mathrm{Vgl}$. beispielsweise Albers (1967a, 202f.), Wissenschaftlicher Beirat beim BMF (1971, 527f.) und o.V. (1991, 17-19). 
daß lediglich Aufwendungen in Höhe des Existenzminimums von Kindern im Rahmen einer steuerlichen Referenzlösung zu berücksichtigen sind. Der Betrag (C) tritt dann an die Stelle von (B). Aus rein steuerlicher Sicht ist einer Berücksichtigung lediglich existentiell notwendigen Bedarfs zuzustimmen, jedoch stehen dem die zivilrechtlichen Bestimmungen entgegen, die zwangsläufig höhere Aufwendungen zur Folge haben. Sollte jedoch - so Tipke $(1984,128)$ resignierend - allenfalls das Zivilrecht an das Steuerrecht angepaßt werden, dann ließe sich gegen eine Lösung nach (C) nichts mehr einwenden. Jedenfalls entspricht (C) dem derzeit verfassungsrechtlich abgesicherten Minimalbetrag.

$\mathrm{Da}$ durch die vorgeschlagene Lösung bei niedrigem Einkommen der Eltern Kindergeld in Höhe des Existenzminimums der Kinder gezahlt wird, wird die Sozialhilfe im Regelfall von der Aufgabe befreit, den Grundbedarf von Kindern abzusichern 105. Dies hat die sehr begrüßenswerte Folge, daß die Einkommensposition von Eltern aufgrund der Unterhaltsverpflichtungen für Kinder nicht mehr unter die Armutsgrenze abrutschen kann 106 . Immerhin sind derzeit - die Zahlen beziehen sich auf 1989 - 32,7\% der Haushalte von Empfängern laufender Hilfe zum Lebensunterhalt Haushalte mit Kindern, denen weit über die Hälfte (ca.

$105 \mathrm{Da}$ durch das Kindergeld auch nicht in den Regelsätzen enthaltene Leistungen der Sozialhilfe, die pauschal für die gesamte Familie gezahlt werden, wie z.B. für Unterkunft und Heizung, abgegolten werden, ist deren Anteil für eine Verrechnung im Rahmen eventueller Sozialhilfezahlungen an die Eltern gesondert auszuweisen. - Auf die implizierten Verschiebungen von Ausgabelasten von den Gemeinden zum Bund soll hier nicht weiter eingegangen werden, da diese prinzipiell im Finanzausgleich berücksichtigt werden können. Dieser Aspekt erschwert allerdings die politische Umsetzung von Reformvorschlägen.

106 Hier wird allerdings bei gegebenem Elterneinkommen argumentiert. Um auch die Auswirkungen von Kindern auf die Erwerbstätigkeit und damit die Erwerbseinkommen der Eltern einigermaßen aufzufangen, ist die Zahlung eines Erziehungsgeldes in den ersten Lebensjahren eines Kindes unerläßlich, und zwar unter dem Aspekt der Vermeidung von Armut insbesondere an Alleinerziehende (vgl. Arbeitsgemeinschaft "Riedmüller/ Glatzer/Infratest", 1991, 61f.). 
58\%) der Empfänger von Hilfe zum Lebensunterhalt angehören 107. Eltern werden durch die vorgeschlagene Lösung - jedenfalls bei gegebenem Einkommen - nicht mehr aufgrund der gesellschaftlich anerkennenswerten Leistung, die Verantwortung für das Aufziehen von Kindern übernommen zu haben, zu Sozialhilfefällen. Abwertende Aussagen dahingehend, beim Kindergeld handele es sich um "Staatsalimente" und es schwäche den Leistungswillen (Dornbusch, 1982, 546), können daher nur als zynisch empfunden werden. Auch wird durch eine solche Lösung nahezu jedem Erwerbsfähigen die Sicherung des Lebensunterhalts aus eigener Kraft ermöglicht, da nach der vollen Übernahme kindesbedingter Belastungen durch das Kindergeld der noch verbleibende Bedarf auf eine erreichbare Höhe reduziert ist 108 . Vielen Eltern (und Kindern!) wird eine Stigmatisierung als Sozialhilfeempfänger erspart und gleichzeitig wieder eine Perspektive für Verbesserungen der persönlichen materiellen Situation zurückgegeben. Andere Vorschläge zur Erhöhung des Anreizes zu eigener Erwerbstätigkeit von Sozialhilfeempfängern gehen völlig am Problem familienlastenbedingter Sozialhilfebedürftigkeit vorbei, sind allerdings um den Preis verfassungsrechtlicher Bedenklichkeit nicht mit zusätzlichen Ausgaben, sondern allenfalls mit Einsparungen verbunden 109 .

\subsection{Zusätzliche Berücksichtigung des Kinderbetreuungsaufwands}

Bisher wurde nur die Belastung der Eltern durch den Sachbedarf des Kindesunterhalts betrachtet. Es stellt sich die Frage, ob ergänzend auch der Betreuungsaufwand für Kinder einkommensteuerlich zu berücksich-

107 Vgl. Deininger (1991, 457-459). Bezogen auf die Altersgruppe von Haushaltsvorständen im erwerbsfähigen Alter wären die Zahlen noch weit höher; hinzu kommt, daß die genannten Zahlen einen steigenden Trend aufweisen.

108 Auch dann verbleiben noch Problemgruppen, insb. Alleinerziehende und Arbeitslose, deren vorhandene finanzielle Absicherung dann aber mit erheblich größerer Wahrscheinlichkeit ausreichend ist.

109 Vgl. beispielsweise die dahingehenden Vorschläge von Leibfritz/Parsche (1988, VI*, 195f.), die eine hälftige Kürzung der Sozialhilfe für erwerbsunwillige Personen vorsehen. 
tigen ist. Eine diesbezügliche Regelung sollte allerdings auf die Altersspanne von der Geburt bis etwa zur Einschulung oder zum 10. Lebensjahr beschränkt werden, die nochmals in die Abschnitte bis zum 3. und ab dem 4. Lebensjahr unterteilt werden könnte ${ }^{110}$.

Dabei ist zu unterscheiden, ob

(1) ein zusätzlicher Steuerfreibetrag generell oder nur bei Erwerbstätigkeit der Eltern zu gewähren ist,

(2) ein jeweils gleicher Betrag (ohne Einzelnachweis von Kosten) oder ein Betrag in Höhe der tatsächlich entstandenen Aufwendungen (ggf. beschränkt durch eine Obergrenze) eingeräumt werden soll,

(3) ein Kinderbetreuungsfreibetrag mit der Zahl der Kinder ansteigen soll.

$\mathrm{Zu}$ (1): Kinderbetreuungsaufwand entsteht grundsätzlich allen Eltern, was für eine generelle steuerliche Berücksichtigung spricht111. Eine steuerliche Berücksichtigung von Opportunitätskosten ist jedoch im Rahmen einer Ist-Einkommensteuer nicht möglich: Die Kinderbetreuung geht in den Fällen, in denen zumindest ein Elternteil nicht erwerbstätig ist, lediglich auf Kosten der Freizeit, deren frei verfügbare Höhe auch in anderen Fällen einkommensteuerlich nicht - oder jedenfalls nicht befriedigend - berücksichtigt werden kann. Die Beschäftigung mit Kindern ist daher, sofern sie auf Kosten der Freizeit geht, vor allem Ausdruck der persönlichen Lebensgestaltung der Eltern, deren Entscheidung für Kinder untrennbar die Verpflichtung zu persönlicher Zuwendung mit einschließt.

110 Die Regelung für die "Große Witwen(r)rente" in der gesetzlichen Rentenversicherung, die von einer vollen Beanspruchung durch Kindererziehung bis zum 18. Lebensjahr des Kindes ausgeht, ist übertrieben.

111 So Dornbusch $(1982,546)$ sowie unter Vereinfachungsgesichtspunkten Kanzler (1988, 213f.). 
In den Fällen der Erwerbstätigkeit der Eltern weisen die Kinderbetreuungsaufwendungen (i.d.R. in Form von monetären Belastungen) dagegen die wesentlichen Merkmale von Betriebsausgaben bzw. Werbungskosten auf, da sie direkt durch die Erwerbstätigkeit der Eltern verursacht werden 112 . Gleichwohl sind auch erwerbstätige Eltern durch eine Kinderbetreuung durch Dritte nicht von ihren elterlichen Pflichten entbunden und daher zusätzlich in der ihnen verbleibenden Freizeit belastet.

Eine Differenzierung zwischen diesen beiden Fällen scheint daher angebracht.

$\mathrm{Zu}$ (2): Die Höhe der anzuerkennenden Kosten sollte sich nach den üblichen Kosten der Betreuung von Kindern in Krabbelstuben, Kindergärten usw. richten, die als Pauschalbeträge, ggf. nach dem Alter der Kinder differenziert, vorgegeben werden können. Die Mehrkosten besonders aufwendiger Kinderbetreuung sollten aus Gründen der sozialen Gleichbehandlung nicht abzugsfähig sein, womit allerdings von dem sonst bei der Anerkennung von Betriebsausgaben und Werbungskosten üblichen Grundsatz, die tatsächlich entstandenen Kosten anzuerkennen, abgewichen wird. Aus Gründen der Praktikabilität ist eine Differenzierung nach der Betreuungsart (entgeltlich oder unentgeltlich, z.B. durch Groß-

112 Vgl. §§ 4(4), 9(1) EStG; auch § 12 EStG sollte dem nicht entgegenstehen. Das Bundesverfassungsgericht entschied zunächst ablehnend $(1978,1)$, um dann berufsbedingten Betreuungsaufwand für Alleinerziehende - ggf. wohl auch im Rahmen der außergewöhnlichen Belastungen grundsätzlich anzuerkennen (1983, 349f.). Die Begründung, daß gleiche Aufwendungen von Doppelverdiener-Ehepaaren aus dem höheren Familieneinkommen bestritten werden können $(1983,348)$ ist - auch unter Berücksichtigung der sonstigen Rechtsprechung des Bundesverfassungsgerichts selbst (1991a, 89) - wenig stichhaltig. Der Verdacht einer Neuauflage des "Edukationseffekts" (vgl. Bundesverfassungsgericht, 1957, 79-82) drängt sich auf, wenn die Frage nach der Notwendigkeit einer Erwerbstätigkeit (Bundesverfassungsgericht, 1983, 348f.; 1985a, 154f.) gestellt wird (in diesem Sinne bereits Simon, 1978, 44-46). 
eltern) wenig erstrebenswert 113 . Wegen der Nähe zu den Werbungskosten bzw. Betriebsausgaben ist ein Verweis auf eine den Eltern zumutbare Eigenbelastung (vgl. $\S \S 33,33 \mathrm{c} \mathrm{EStG}$ ) wenig sachgerecht 114 .

$\mathrm{Zu}$ (3): Bei einer Orientierung der Höhe einer Pauschale an Aufwendungen, die eine Kinderbetreuung typischerweise bei Unterbringung in einer Krabbelstube, einem Kindergarten o.ä. mit sich bringt, liegt es nahe, einen Pauschalbetrag pro Kind vorzusehen. Nur bei einer Orientierung an den Kosten einer Unterbringung zuhause - wovon aber bisher nicht ausgegangen wurde -, wäre davon abweichend anzunehmen, daß eine Betreuungsperson auch mehrere Kinder übernehmen könnte.

Es spricht also einiges für die Gewährung eines Pauschalbetrages pro Kind im entsprechenden Alter, sofern kein Elternteil für die Betreuung zur Verfügung steht. Das ist dann der Fall, wenn in der sog. Vollfamilie beide Eltern erwerbstätig sind (dieser Fall wird im geltenden Einkommensteuerrecht nicht berücksichtigt) oder Erwerbstätigkeit bei Alleinerziehenden vorliegt. Von Erwerbstätigkeit kann etwa bei einer regelmäßigen Wochenarbeitszeit von der Hälfte der üblichen (Vollzeit-) Wochenarbeitszeit von Arbeitnehmern gesprochen werden. Diese Regelung könnte für Doppelverdiener-Ehepaare wegen des gleichwohl noch höheren zeitlichen Spielraums restriktiver als für Alleinerziehende gefaßt werden. Beispielsweise könnte für Alleinerziehende bereits eine geringere regelmäßige Wochenarbeitszeit ausreichen oder bei Doppelverdienern eine Gesamtarbeitszeit von mindestens dem 1,5-fachen der üblichen (Vollzeit-)Wochenarbeitszeit verlangt werden. Einer Erwerbstätigkeit gleichgestellt werden sollten Fälle, in denen eine Kinderbetreuung durch

113 Denn selbst bei "kostenfreier" Betreuung z.B. durch die Großeltern entstehen Fahrtkosten und weitere Kosten bei den Betreuenden. Was noch schwerer ins Gewicht fallt, sind jedoch die dann auftretenden Probleme fiktiver Arbeitsverträge. In der Literatur gehen die Meinungen zur Frage Höchst- vs. Pauschbetrag auseinander: Mennel (1974, D188-D193) ist unmißverständlich für einen Pauschbetrag; Lang (1983, 107, 125; 1984, 130f.) scheint sich eher für einen Höchstbetrag auszusprechen, ebenso Deutscher Juristentag (1974, L242) und Bundesverfassungsgericht (1983, 355). Die Regelung in $\S 33 \mathrm{c}$ EStG stellt eine Kombination aus beiden Verfahren dar.

114 So auch Lang $(1984,131)$, Kanzler $(1986 ; 1988,210)$. 
den nicht erwerbstätigen Elternteil aufgrund einer Behinderung oder Krankheit nicht möglich ist (so auch $\S 33 \mathrm{c}(5) \mathrm{EStG}$ ) ${ }^{115}$.

Komplikationen durch fiktive Arbeitsverträge zur Kinderbetreuung sind bei der vorgeschlagenen Regelung durch Pauschbeträge nicht zu fürchten 116. Problematisch bleibt allerdings die Bestimmung der Wochenarbeitszeit bei bestimmten Beschäftigtengruppen, insbesondere bei Selbständigen. Hier kann hilfsweise, wie auch im Erziehungsgeldgesetz (§ 2 BErzGG; Zmarzlik/Zipperer/Viethen, 1986, 376, Rz.12-14), auf die üblicherweise für eine vereinbarte Arbeitsleistung benötigte Arbeitszeit abgestellt werden.

Eine Integration von einkommensteuerlicher Berücksichtigung der Kinderbetreuungsaufwendungen und Erziehungsgeld - so wie sie oben für Kinderfreibetrag und Kindergeld gefordert wurde - ist nicht erstrebenswert, da die Zielsetzungen beider Maßnahmen unterschiedlich, in gewissem Sinne sogar konträr sind: Mit der einkommensteuerlichen Regelung soll den tatsächlichen Belastungen einer Kinderbetreuung durch Dritte Rechnung getragen werden, womit als Nebeneffekt die Erwerbstätigkeit der Eltern gefördert bzw. eine Diskriminierung erwerbstätiger Eltern beseitigt wird, während durch das Erziehungsgeld gerade die Kinderbetreuung durch die Eltern finanziell ermöglicht und damit gefördert werden soll. Eine Abstimmung der jeweils verwendeten Definition von Erwerbstätigkeit ist allerdings erforderlich, um sonst mögliche zu starke Grenzbelastungen zusätzlicher Erwerbstätigkeit eines Elternteils oder ungerechtfertigte Doppel-Begünstigungen zu vermeiden.

\subsection{Zur Besteuerung der Kinder}

Die Gewährung von Kinderfreibeträgen bzw. von Kindergeld an die Eltern geht davon aus, daß diese für den Unterhalt ihrer Kinder aufkommen. Damit ist das Existenzminimum der Kinder bereits bei ihren

115 Die Aufwendungen haben dann allerdings eher den Charakter außergewöhnlicher Belastungen als den von Betriebsausgaben bzw. Werbungskosten.

116 Vgl. zu solchen Gefahren Kanzler $(1988,211)$. 
Eltern berücksichtigt. Sofern Kinder über eigenes Einkommen verfügen, ist die zusätzliche Gewährung eines eigenen Existenzminimum-Grundfreibetrages nicht angebracht 117 .

Nach derzeitigem Recht erhalten Kinder mit eigenem Einkommen auch dann, wenn ihre Eltern einen Kinderfreibetrag in Anspruch nehmen, den vollen Grundfreibetrag. Gleichzeitig führen eigene Einkünfte der Kinder im Normalfall nicht zu einer Kürzung des Kinderfreibetrags bzw. des Kindergeldanspruchs der Eltern. Lediglich der zusätzlich gewährte Ausbildungsfreibetrag wird um eigene Einkünfte des Kindes gekürzt, sofern diese den Betrag von 3600 DM übersteigen (vgl. § 33a(2) EStG). Ein Kindergeldanspruch für in einer Ausbildung befindliche Kinder erlischt, wenn die Ausbildungsvergütung (nicht etwa die Summe der Einkünfte!) des Kindes den Betrag von $750 \mathrm{DM}$ pro Monat übersteigt (§ 2(2) BKGG).

Lösungsmöglichkeiten bestehen sowohl in einer gemeinsamen Veranlagung von Eltern und Kindern als auch in der Berücksichtigung innerfamiliärer Transfers bei individueller Veranlagung. Gegen die erste der beiden Möglichkeiten spricht allein schon die Art der Eltern-Kind-Beziehung (Unterhaltsgemeinschaft, nicht Erwerbsgemeinschaft), außerdem muß eine steuerliche Diskriminierung der Familie ausgeschlossen werden 118 und treten erhebliche Probleme bei der Umsetzung einer gemeinsamen Veranlagung auf (Royal Commission on Taxation, 1966, Bd.3, 122-125, 130-141; Steuerreformkommission, 1971, 196-199). Die zweite Möglichkeit verwendet dagegen alle vorhandenen Informationen über die personelle Einkommensaufteilung, vermeidet zahlreiche Abstimmungsprobleme für den Fall der Beendigung der gemeinsamen

117 Vgl. hierzu allgemein z.B. Pohmer $(1968,161)$. Anderer Ansicht, allerdings wenig überzeugend, Steuerreformkommission (1971, 206, Rz.628).

118 Vgl. Bundesverfassungsgericht (1965) vom 30.6.1964. Verfassungsrechtlich unbedenklich wäre in dieser Hinsicht möglicherweise eine Option auf getrennte Veranlagung. Damit würden jedoch zugunsten einzelner Steuerpflichtiger Abweichungen von der Norm der gemeinsamen Veranlagung ermöglicht, was meines Erachtens den Gleichbehandlungsgrundsatz in seinem ökonomischen Gehalt verletzt. 
Veranlagung und paßt insgesamt in ein individualorientiertes Gesamtkonzept.

Dem Grundprinzip - Erfassung der tatsächlichen individuellen Leistungsfähigkeit - kann entsprochen werden, indem den Eltern aufgrund ihrer kindesbedingten Belastungen ein Kinderfreibetrag119 eingeräumt wird, gleichzeitig allerdings bei den Kindern Einkünfte in dieser Höhe verbucht werden. Bei Fehlen sonstiger Einkünfte der Kinder entstehen keine Steuerzahlungsverpflichtungen, da ein Grundfreibetrag auch bei der Veranlagung der Kinder zu berücksichtigen ist. Verfahrenstechnisch wäre in der Regel keine gesonderte Steuererklärung der Kinder erforderlich, eine entsprechende Angabe in der Steuererklärung der Eltern würde genügen.

Liegen darüber hinaus Einkünfte des Kindes vor, aus denen eine eigene Finanzierung des Lebensunterhalts möglich ist ${ }^{120}$, dann sind die zwangsläufigen Unterhaltsaufwendungen der Eltern entsprechend geringer anzusetzen, was zur Folge hat, daß sowohl der Kinderfreibetrag der Eltern als auch die beim Kind zu erfassenden Übertragungen vermindert werden. Zunächst wird daher auch in diesem Fall noch keine Steuerzahlung des Kindes ausgelöst, und es ist noch keine gesonderte Steuererklärung des Kindes erforderlich. $\mathrm{Zu}$ einer positiven Steuerschuld des Kindes kommt es erst dann, wenn die eigenen Einkünfte das Existenzminimum übersteigen, womit dann eine steuerliche Berücksichtigung bei den Eltern nicht mehr erforderlich ist und daher entfallen kann.

In der bisherigen Argumentation wurde davon ausgegangen, daß der zu berücksichtigende Kinderfreibetrag dem Existenzminimum des Kindes entspricht. Ein anderes Ergebnis, nämlich gleichzeitig steuerliche Berück-

119 Dieser kann auch durch ein Kindergeld - wie oben beschrieben - ersetzt oder nach der Höhe des Elterneinkommens differenziert werden, worauf später noch eingegangen wird. Zunächst ist jedoch die rein steuersystematische Argumentation mit einem Kinderfreibetrag in Höhe des Existenzminimums leichter nachvollziehbar.

120 Dies ist dann der Fall, wenn Kinder über eigene Vermögenserträge oder Arbeitseinkünfte verfügen; vgl. §§ 1602, 1649 BGB sowie Schlüter (1991, 165-169). Sofern Kinder über Vermögen verfügen, entfällt darüber hinaus die sog. gesteigerte Unterhaltspflicht der Eltern. 
sichtigung des Kindes bei seinen Eltern und Steuerzahlungsverpflichtung des Kindes, kann allerdings dann eintreten, wenn der zu berücksichtigende Kinderfreibetrag wegen einer Orientierung an den zivilrechtlichen Bestimmungen oberhalb des Existenzminimums liegt, was dann aber auch folgerichtig ist. Komplikationen treten auch hier nicht auf, allerdings steigt die Zahl der - u.U. in einem vereinfachten Verfahren - notwendigen Steuerveranlagungen 121 .

Weiterhin ist es sachgerecht, wenn bei Staffelung der Kinderfreibeträge nach dem Alter der Kinder die Existenzminimum-Grundfreibeträge bei eigener Veranlagung der Kinder ebenfalls nach dem Alter gestaffelt werden. Dies ist am einfachsten durch eine Ausgliederung der Existenzminimum-Freibeträge aus dem Tarif zu erreichen, wo diese dann unter Berücksichtigung des Alters übersichtlich geregelt werden können 122 .

Bei einem Abgehen von einer rein steuersystematischen Lösung durch Ersetzung von Kinderfreibeträgen durch ein Kindergeld, wie dies weiter oben gefordert wurde, ist eine etwas modifizierte Vorgehensweise erforderlich. Das an die Eltern zu zahlende Kindergeld ist bei eigenen Einkünften des Kindes dann in dem Umfang zu kürzen, in dem das Kind selbst für seinen Lebensunterhalt aufkommen kann 123 .

121 Bei minderjährigen Kindern ist prinzipiell eine Lösung wie von Lang (1983, 125) vorgeschlagen möglich, nach der die Unterhaltsbezüge im Rahmen eines Familiensteuerbescheids von der unterhaltenden Person stellvertretend für die unterhaltene Person versteuert werden. Fraglich bleibt allerdings, wer die fällige Steuerschuld auf den Kindesunterhaltsbetrag im zivilrechtlichen Vergleich zu tragen hat.

122 Vgl. hierzu Langs Vorschläge zu "Grundbedarfspauschalen" (Lang, 1985, 99f.; 1990, 339). Zu anderen Gründen für eine solche Ausgliederung aus dem Tarif vgl. Haller (1981b, 12-14), Dziadkowski (1986, 508f.) sowie Karl-Bräuer-Institut des Bundes der Steuerzahler (1991, 81f.).

123 Präziser ausgedrückt im Verhältnis eigene Einkünfte abzüglich Werbungskosten zu Existenzminimum-Grundfreibetrag des Kindes. Sofern die steuerlich zu berücksichtigenden Unterhaltszahlungen oberhalb des Existenzminimums liegen, sind diese im Nenner anzusetzen. 
Im Vergleich zur derzeitigen Regelung wird der Anreiz zu eigener Erwerbstätigkeit der Kinder gemindert, soweit diese von der doppelten Berücksichtigung ihres Grundbedarfs profitieren. Dies ist jedoch vertretbar, zumal auch nach einer Neuregelung im hier vorgeschlagenen Sinne durch die Gewährung von Werbungskosten-Pauschalen geringe Kindeseinkünfte unberücksichtigt bleiben. Wichtiger ist jedoch, daß durch eine Reform eine in sich konsistentere und damit gerechtere Einkommensbesteuerung ermöglicht wird, die außerdem - soweit dies nicht bereits durch andere Maßnahmen verhindert wird124 - wesentlich dazu beiträgt, daß Verlagerungen von Einkunftsquellen auf Kinder zu Zwecken der Steuerersparnis weniger lohnend werden 125 .

\section{Reduktion von Manipulationsmöglichkeiten}

Im Rahmen einer progressiven Einkommensteuer können interpersonelle Verlagerungen der Steuerbemessungsgrundlage zu einer Reduktion der Steuerschuld führen. Soweit der Gesetzgeber Unterhaltszahlungen als Minderung der steuerlichen Leistungsfähigkeit der unterhaltenden Person anerkennt, liegt dies auch explizit in seiner Absicht.

Entgegen den Absichten des Gesetzgebers sind darüber hinausgehende Verlagerungen im Rahmen zivilrechtlicher Verträge möglich, wenn der in der Regel zwischen Vertragspartnern zu unterstellende Interessengegensatz fehlt. Vor allem für Verträge zwischen Familienangehörigen ist diese Möglichkeit in Betracht zu ziehen.

Die entsprechenden Verträge sind dann danach zu differenzieren, ob sie entgeltliche oder unentgeltliche Leistungen zum Inhalt haben. Entgeltliche Leistungen sind einkommensteuerlich wie in Verträgen unter Fremden anzuerkennen, bei unentgeltlichen Leistungen handelt es sich um Schenkungen, die als solche steuerlich zu erfassen sind (Söffing, 1979, 321-330). Während entgeltliche Leistungen beim Übertragenden als

124 Vgl. die Vorschläge zur steuerlichen Erfassung von Schenkungen in Kapitel VI.7.

$125 \mathrm{Zu}$ Steuermanipulationsmöglichkeiten, die noch aufgrund des verbleibenden Progressionsvorteils attraktiv sein können, vgl. Kapitel VI.5. 
Werbungskosten oder Betriebsausgaben abzugsfähig sein können, ist dies bei unentgeltlichen Leistungen nicht der Fall, da es sich offensichtlich um Einkommensverwendung handelt. Vertragliche Leistungen zu marktunüblichen Sonderkonditionen sind gegebenenfalls in einen Entgeltanteil und einen Transferanteil aufzuspalten (Söffing, 1979, 360-374).

Die unentgeltliche Übertragung von Vermögensgütern bringt für den Übertragenden regelmäßig das Risiko mit sich, die eigentumsrechtliche Kontrolle über das Vermögensgut aufzugeben. Eine seinen Absichten entsprechende Verwendung des Empfängers ist nicht sichergestellt. Daher ist die Übertragung von Nutzungsrechten, die dieses Risiko vermeidet, besonders beliebt (Altfelder, 1987, 132-134). Hier werden lediglich die Erträge transferiert, nicht jedoch die Ertragsquelle. Bei der unentgeltlichen Übertragung eines Nutzungsrechts kann es wegen der dabei für steuerliche Zwecke auftretenden Bewertungsproblematik zweckmäßig sein, nicht den Transfer des Nutzungsrechts selbst, sondern die laufenden Erträge aus der Übertragung dieses Rechts als einkommensteuerlichen Anknüpfungspunkt zu wählen. Das bedeutet, daß eine laufende Zurechnung der Erträge zum Eigentümer vorgenommen wird, der diese als Transfer an den Nutzungsberechtigten weiterleitet ${ }^{126}$. Auf eine Erfassung und Bewertung des Nutzungsrechts selbst kann dann verzichtet werden.

Bei Arbeitsverträgen unter Angehörigen ist glaubhaft zu machen, daß tatsächlich Leistungen erbracht werden und die vereinbarten Konditionen angemessen sind. Die Begründung von Scheinarbeitsverhältnissen läßt sich kaum völlig verhindern, ist jedoch wegen der restriktiven Rahmenbedingungen (z.B. eigener Betrieb des Transferleistenden, fachliche Qualifikation des Transferempfängers und Schein-Arbeitnehmers verbunden mit tatsächlicher Erwerbslosigkeit) auf einen sehr kleinen Personenkreis begrenzt. Betriebsprüfungen könnten durch Einsichtnahme in

126 Auf eine entsprechende Praxis des Bundesfinanzhofs verweisen Biergans/Stockinger $(1982,29)$. Allerdings geht es im geltenden Steuerrecht weniger um die Lösung von Bewertungsfragen als vielmehr darum, eine durch die meist vernachlässigbare Schenkungsteuer kaum behinderte interpersonelle Verschiebung von Teilen der Einkommensteuer-Bemessungsgrundlage wenigstens in besonders eklatanten Fällen zu verhindern. 
Personalakten und Geschäftskonten sowie Befragung von Mitarbeitern möglichen Mißbrauch weiter reduzieren.

Als ideale einkommensteuerliche Lösung zur Verhinderung schwer nachvollziehbarer tatsächlicher oder fingierter Transaktionen in den beschriebenen Bereichen gilt die Zusammenveranlagung von Familienangehörigen zur Einkommensteuer. Besondere Vertragsgestaltungen können dann keine einkommensteuerlichen Folgen haben, weil die individuellen Steuerbemessungsgrundlagen erst gar nicht ermittelt werden müssen. Immer, wenn von einer gemeinsamen Veranlagung der Familienangehörigen abgegangen wird, besteht die Möglichkeit der beschriebenen Steuermanipulationen, die einen Verstoß gegen das Gebot der Steuergerechtigkeit darstellen (Pohmer, 1968, 164f.; Albers, 1980, 195; Kassella/Spahn, 1990, 2f.). Die Anwendungsmöglichkeiten einer gemeinsamen Veranlagung enden jedoch spätestens mit der Gründung eines eigenen Hausstands von Kindern bzw. mit deren Volljährigkeit (Royal Commission on Taxation, 1966, Bd.3, 136-139). Daher bietet auch die gemeinsame Veranlagung kein generell gültiges Mittel zum Ausschluß von Manipulationsmöglichkeiten, sondern stellt lediglich ein zeitlich befristet wirkendes Arrangement dar.

Im derzeitigen deutschen System findet die gemeinsame Veranlagung faktisch bei der Ehegattenbesteuerung Anwendung; wegen des Ausgleichs der individuellen Bemessungsgrundlagen durch das Splitting werden die angesprochenen Manipulationsmöglichkeiten unter rein steuerlichen Aspekten weitgehend sinnlos. Sie sind daher vor allem für Übertragungen von Eltern auf Kinder interessant 127 . Dies liegt im Rahmen der Übertragung fundierter Einkünfte vor allem darin begründet, daß aufgrund der vernachlässigbaren Belastung durch die Schenkungsteuer ein Transfer von Vermögen ohne nennenswerte Steuerbelastung möglich ist. Für die steuerliche Vorteilhaftigkeit solcher Transfers genügen daher bereits relativ geringe Unterschiede in der marginalen Belastung mit Einkommensteuer. Durch eine Integration der Erbschaft- und Schenkungsteuer in die Einkommensteuer werden solche Vorteile nahezu ausgeschlossen. Mani-

127 Zur steuerlichen Vorteilhaftigkeit von innerfamiliären Einkünfteverlagerungen bei geltendem Recht vgl. Altfelder (1987, 125-135). 
pulationen im Bereich fundierter Einkünfte werden dann aus steuerlichen Gründen unattraktiv, zumal wenn die Reduktion der Verfügungsmacht beim Transferleistenden sowie Transaktionskosten (z.B. Notariatskosten) in Rechnung gestellt werden. Bei entsprechenden Reformen ist daher auch eine stärkere Hinwendung zu einer individuellen Besteuerung möglich, ohne daß dadurch bestimmte Gruppen von Steuerpflichtigen aufgrund von besonderen Gestaltungsmöglichkeiten profitieren.

\section{Versicherungen zugunsten Dritter}

Im allgemeinen rechtfertigen Versicherungen zugunsten Dritter genausowenig einen Abzug von der Steuerbemessungsgrundlage des Leistenden wie direkte Transferzahlungen. Unter diesem Aspekt wurde auch schon zu einigen Formen der Altersvorsorge zugunsten Dritter Stellung genommen 128 .

Sofern allerdings zwangsläufige Unterhaltsverpflichtungen zugunsten Dritter einkommensteuerlich anerkannt werden, liegt es nahe, deren Absicherung gegen Risikofälle in gleicher Weise zu berücksichtigen. $\mathrm{Zu}$ nennen ist hier zunächst die Absicherung des Unterhaltsverpflichteten selbst gegen Einkommensausfall, womit indirekt auch dessen Leistungen an Unterhaltsberechtigte gesichert werden. Solche Versicherungen wurden bereits abschließend behandelt ${ }^{129}$. Gesondert zu behandeln sind daher lediglich Versicherungen, die im Todesfall des Unterhaltsverpflichteten eine Aufrechterhaltung von Unterhaltsleistungen garantieren. Wird eine Vorsorge als angemessen erachtet, dann sollte ein Abzug von Beitragsleistungen von der Steuerbemessungsgrundlage ermöglicht werden; ausgezahlte Leistungen sind in die Steuerbemessungsgrundlage des Empfängers einzubeziehen.

Bei Eintritt des Versicherungsfalls wird sich der Umfang von Leistungen kaum am konkreten Bedarf von Unterhaltsberechtigten, insbesondere unter Anrechnung eigenen Einkommens, sondern lediglich im Rahmen einer Typisierung von Bedarfssituationen an der Höhe gezahlter

128 Vgl. Kapitel V.1.3.5.

129 Vgl. Kapitel V.1 und V.2. 
Beiträge ausrichten können. Deren Höhe könnte allerdings unter steuerlichen Aspekten innerhalb eines vorgegebenen maximalen Bedarfsrahmens freigestellt werden.

Ziemlich unstrittig ist ein Bedarf von unterhaltsberechtigten Kindern, sofern dieser nicht durch staatliche Leistungen wie das Kindergeld bereits gedeckt wird. Dieser Bedarf kann allerdings in der Regel noch zu 50\% vom verbleibenden unterhaltsverpflichteten Elternteil bestritten werden.

Auch eine Hinterbliebenenleistung an den unterhaltsberechtigte Kinder des Verstorbenen betreuenden Ehegatten erscheint angemessen. Eine Sonderbelastung aufgrund der Kinderbetreuung kann (maximal) bis zum 15. Lebensjahr des jüngsten Kindes angenommen werden, allerdings etwa zwischen dem 10. und 15. Lebensjahr nur noch in reduziertem Umfang. Ein Bedarf besteht dabei maximal bis zur Höhe von 50\% des Einkommens des verstorbenen Ehepartners ${ }^{130}$. Ergänzend könnte auch die Betreuungstätigkeit eines nicht erwerbstätigen Elternteils Gegenstand einer Absicherung sein. Sind dagegen keine unterhaltsberechtigten Kinder zu betreuen, dann ist die Annahme eines Bedarfs von Hinterbliebenen im allgemeinen nicht gerechtfertigt. Ein dauerhafter Bestandsschutz für eventuell vorliegende Unterhaltsbeziehungen kommt hier nicht in Betracht; allerdings könnte für einen begrenzten Zeitraum (z.B. zwei Jahre) eine Übergangszahlung geleistet werden. Hierbei sind generell Ausgestaltungen abzulehnen, die eine Leistung vom Verhalten des Bezugsberechtigten abhängig machen, da sie in unangemessener - und sachlich ineffizienter - Weise in die persönlichen Rechte eingreifen (Ausschluß einer Leistungsgewährung bei Wiederheirat) ${ }^{131}$ oder bei längerfristiger Betrachtung zu Fehlsteuerungen führen (Ausschluß von Leistungen bei Erwerbstätigkeit).

130 Abweichend vom Verfahren einer familieninternen personenbezogenen Einkommenszurechnung wäre hier allerdings das "originäre" Einkommen des Verstorbenen zugrunde zu legen. Auch könnte ein Ausgleich für den Wegfall der Haushaltsersparnis berücksichtigt werden.

131 Vgl. hierzu auch die kritische Stellungnahme von Ruland (1973, 378381). 
Die versicherungstechnische Abwicklung des angesprochenen Leistungskatalogs entspricht der einer Todesfallrisikoversicherung (Reuter, 1987, 99). Bei Abschluß einer privaten Versicherung ist dabei die Leistungshöhe in den angedeuteten Grenzen frei variierbar. Bei Einbeziehung in die gesetzliche Rentenversicherung bzw. Beamtenversorgung muß pauschaliert werden, die gegenwärtigen Regelungen (vgl. $\S \S 46,47$ SGB VI) sind nach den hier angestellten Überlegungen entsprechend abzuändern. Einkommensteuerlich ist dann die Abzugsfähigkeit entsprechender Beiträge von der Steuerbemessungsgrundlage bei Steuerpflicht der Bezüge sicherzustellen.

Darüber hinaus besteht Bedarf an einer ausreichenden Alterssicherung beider Ehepartner unabhängig davon, welcher der Ehepartner zuerst stirbt. Es entspricht dabei der bisher vorgeschlagenen Verfahrensweise mit Ausnahme der Gütertrennung hälftige Zurechnung des gemeinsamen Einkommens und auch der Vorsorgeaufwendungen durch das Splitting sowie den sonstigen Regelungen des Steuer- und Zivilrechts, wenn eine hälftige Zurechnung der gemeinsam während der Ehezeit erworbenen Ansprüche vorgenommen wird, womit eine selbständige "Hinterbliebenensicherung" beider Ehepartner erreicht ist ${ }^{132}$. Besondere Leistungen für Hinterbliebene könnten dann, abgesehen von einer zeitlich eng befristeten Übergangsleistung, entfallen, eine weitgehend beitragsäquivalente Ausgestaltung der Alterssicherung wäre möglich. Dazu stehen jedoch die Regelungen der Alterssicherungssysteme in Widerspruch, die am überholten patriarchalischen Eheverständnis festhalten und damit ein Splitting von Ansprüchen verhindern. Unter einkommensteuersystematischen Aspekten müßten im Hinterbliebenenfall diejenigen Alterseinkünfte aufgrund gemeinsam erworbener Ansprüche der Ehegatten, die über $50 \%$ der in der Ehezeit erworbenen Gesamtversorgung hinaus-

132 Vgl. dazu auch Sachverständigenkommission für die soziale Sicherung der Frau und der Hinterbliebenen (1979, 42f.). Um den Aspekt der Haushaltsersparnis zu berücksichtigen, könnte das System für Ehepartner um einen versicherungsmathematisch berechneten Zuschlag für den hinterbliebenen Ehepartner und einen Abschlag für bestehende Ehen modifiziert werden. 
gehen 133 , beim "Transferleistenden" zusätzlich versteuert werden. Eine Steuerpflicht eines entsprechenden Teils der Beiträge ist allerdings nicht durchführbar, weil der Umfang der Hinterbliebenensicherung - allen versicherungstheoretischen Erkenntnissen widersprechend - ehezeitunabhängig und verhaltensabhängig (Scheidung, Wiederheirat) ist, außerdem der für 50\% der Gesamtversorgung notwendige Beitrag wegen der Interdependenz der Sicherung der Ehegatten nicht eigenständig bestimmbar ist. Stattdessen sollte eine Reform der den Grundgedanken des Eherechts widersprechenden Alterssicherungssysteme endlich eingeleitet werden 134 .

\section{Sonderstatus für Erbschaften und Geschenke?}

Erbschaften und Geschenke erhöhen die steuerliche Leistungsfähigkeit des Empfängers, sind also nach der Reinvermögenszugangstheorie in die Einkommensteuerpflicht einzubeziehen, da nur so eine annähernde Gleichmäßigkeit der Besteuerung verwirklicht werden kann. Allerdings finden sich in der Literatur zahlreiche Argumente, die eine Einbeziehung von Erbschaften und Geschenken in die Bemessungsgrundlage der Einkommensteuer ablehnen:

- Der Einwand, eine "steuerliche Behandlung von Vermögen als Einkommen" sei abzulehnen (Steuerreformkommission, 1971, 659, Fn.50), kann sich lediglich auf die Abgrenzung des volkswirtschaftlichen Einkommensbegriffs stützen ${ }^{135}$, ist daher im Rahmen einer personenbezogenen Einkommensteuer gegenstandslos.

- Das Argument, daß die Erben selbst an der Bildung des zu vererbenden Vermögens maßgeblich beteiligt waren (Wissenschaftlicher Beirat

133 Jedenfalls in der gesetzlichen Rentenversicherung sind unter $50 \%$ der Gesamtversorgung bleibende Leistungen nahezu ausgeschlossen, weil $100 \%$ der eigenen Rente plus $60 \%$ der Rente des verstorbenen Partners immer über $50 \%$ der Gesamtversorgung ergeben (Ausnahmen: Wiederheirat und Einkommensanrechnung nach § 97 SGB VI).

134 Für Überlegungen in diese Richtung vgl. auch Sachverständigenkommission für die soziale Sicherung der Frau und der Hinterbliebenen (1979, 33-72).

135 Vgl. Kapitel II.2.9. 
beim BMF, 1967, 383f.; Steuerreformkommission, 1971, 661, Rz.163), läßt sich so pauschal nicht nachvollziehen. Für Ehepartner trägt in der Regel bereits der Zugewinnausgleich ${ }^{136}$ diesem Gedanken Rechnung. Zwischen Eltern und Kindern ist zwar in vielen Fällen ein Leistungsaustausch anzutreffen (Mitarbeit im elterlichen Betrieb, Familienhilfe z.B. beim Bau des Eigenheims, in der Kinderbetreuung und Altenhilfe), dieser Leistungsaustausch läßt sich aber entgeltlich gestalten wie bei der Mitarbeit im elterlichen Betrieb oder seine Richtung ist keineswegs eindeutig von der Kinder- zur Elterngeneration wie bei den genannten innerfamiliären Dienstleistungen. Eine pauschale Berücksichtigung ist daher nicht angebracht; im Einzelfall ist die Vereinbarung eines Entgelts möglich, um eine unangemessene zweifache steuerliche Erfassung zu vermeiden.

- Der grundgesetzlich verankerte "Schutz von Ehe und Familie" (Art. 6 GG) läßt sich nicht in einen unbedingten Schutz des Familienvermögens umdeuten; die Erhöhung steuerlicher Leistungsfähigkeit beim Erben (bzw. Beschenkten) ist zunächst unabhängig von dessen verwandtschaftlicher Beziehung zum Erblasser (bzw. Schenkenden)137. Eine Berücksichtigung der verwandtschaftlichen Beziehungen erfolgt bereits in den bürgerlich-rechtlichen Bestimmungen zur gesetzlichen Erbfolge und der - meines Erachtens im allgemeinen nicht mehr zeitgemäßen - Festlegung von Pflicht-Erbteilen, womit jedoch nicht das Potential an steuerlicher Leistungsfähigkeit, sondern nur deren Zuordnung berührt wird.

136 Dessen erbrechtliche Realisierung beinhaltet zudem regelmäßig eine Überkompensation und entspricht damit nicht den Grundgedanken der Zugewinngemeinschaft. Die entsprechenden Regelungen sind an diejenigen des Zugewinnausgleichs im Scheidungsfall anzupassen. Zur derzeitigen Regelung vgl. D.Schwab (1991, 127-130).

137 Vgl. hierzu Wissenschaftlicher Beirat beim BMF, 1967, 383; anderer Ansicht: Steuerreformkommission, 1971, 661, Rz.164; Karl-BräuerInstitut des Bundes der Steuerzahler, 1971, 181; OECD, 1988b, 102f., Rz.2.41. 
- Die Versorgung von Hinterbliebenen138 spricht ebenfalls nicht für eine Sonderbehandlung von Erbschaften und Geschenken, da die besonderen Instrumente einer Versorgung von Angehörigen im Bedarfsfall nach den hier gemachten Vorschlägen 139 ohnehin nicht in die Erbmasse eingerechnet werden, sondern erst die Zuflüsse aus entsprechenden Versicherungen einkommensteuerpflichtig sind. Wurde vom Erblasser keine Vorsorge für Hinterbliebene vorgenommen, dann ist die Möglichkeit einer nachträglichen Vorsorge zu Lasten der Erbmasse einzuräumen.

- Familienunternehmen sehen sich im Todesfall des Eigentümers aufgrund der anfallenden Erbschaftsteuer und eventuellen Erbauseinandersetzungen besonderen Liquiditätsproblemen ausgesetzt. Sofern eine Weiterführung rentabel ist, sollte diese aus erbschaftsteuerlichen Gründen nicht in Frage gestellt werden. Hierzu genügt ein Anrecht auf zeitlich befristete verzinsliche Stundung der Steuerschuld. Eine Steuerermäßigung für Familienunternehmen im Erbschaftsfall - auch in Form einer unverzinslichen Stundung der Steuerschuld ( $\$ 28$ ErbStG) - führt lediglich zu unangemessenen Subventionierungen. Sollte aus wettbewerbspolitischen Gründen eine besondere Förderung von kleinen und mittleren Unternehmen erwünscht sein, so ist eine Übernahme im Erbschaftsfall nicht anders wie eine Übernahme, Beteiligung oder Neugründung in anderen Fällen zu fördern.

- Durch die Besteuerung von Erbschaften und Geschenken ist die intertemporale Neutralität der Besteuerung immer dann verletzt, wenn die Nutzen der Erben (Beschenkten) Gegenstand der Nutzenfunktion des Erblassers (Schenkenden) sind (Huber, 1991/92, 442f.). Die Einkommensteuer verfügt jedoch als personenbezogene Steuer grundsätzlich nicht über die Eigenschaft einer objektbezogenen Neutralität 140 .

138 Vgl. auch Wissenschaftlicher Beirat beim BMF, 1967, 386; Steuerreformkommission, 1971, 671-673; Karl-Bräuer-Institut des Bundes der Steuerzahler, 1971, 178.

139 Vgl. Kapitel VI.6.

140 Vgl. auch R.A.Musgrave (1985, 151f.) sowie Kapitel II.2.7. 
- Damit zusammenhängend: Die Besteuerung von Erbschaften und Geschenken kann die Arbeits- und Sparanreize des Erblassers verringern (Karl-Bräuer-Institut des Bundes der Steuerzahler, 1971, 182). Deshalb sollte jedoch nicht von der auch unter allokativen Gesichtspunkten günstigen Konzeption einer umfassenden Einkommensbesteuerung abgegangen werden. Auch werden die entsprechenden Anreize der Erben durch die Steuererhebung und die damit verbundene Reduktion des Dispositionsspielraums vergrößert.

- Bei den typischen Erbgängen von einer Generation zur nächsten können dann Härtefälle auftreten, wenn zunächst ein überlebender Ehepartner die Position des Vorerben (nicht nur im engen juristischen Sinne) einnimmt, so daß unter Umständen in kurzer Abfolge das gleiche Vermögen zweimal Gegenstand eines Erbfalls wird ${ }^{141}$. Auch mag sich der überlebende Ehepartner lediglich als treuhänderischer Verwalter des Erbes fühlen. Insofern erscheint es angemessen, die Steuerschuld auf das Erbe eines Ehegatten in gewissem Umfang von dessen Lebensdauer über den Tod des Erblassers hinaus abhängig zu machen ${ }^{142}$. So ist es vorstellbar, die Steuerschuld in jährlichen Raten über einen Zeitraum von z.B. 20 Jahren hinweg zu erheben, bei vor Ablauf dieses Zeitraums eintretendem Tod des bis dahin überlebenden Ehepartners die noch ausstehenden Raten jedoch verfallen zu lassen 143 . Eine solche Regelung stellte eine insgesamt vertretbare Konzession in bezug auf den oben angesprochenen Sachverhalt der tatsächlichen Nutzung gestiegener steuerlicher Leistungsfähigkeit dar. Sie ist jedenfalls anderen Regelungen wie Sonderfreibeträgen und reduzierten Steuersätzen für den überlebenden Ehepartner überlegen; auch komplizierte Anrechnungsverfahren beim nächsten Erbgang

141 Die Aussage des Wissenschaftlichen Beirats beim BMF (1967, 389), daß "regelmäßig Perioden mit einer überdurchschnittlich langen Frist bis zum nächsten Erbgang folgen", gilt dagegen nur für die typische Erbfolge von Generation zu Generation.

142 Die Problematik sieht auch Kisker (1964, 132f.).

143 Äquivalent hierzu ist eine sofortige Erhebung der Steuerschuld verbunden mit einer partiellen Rückerstattung, sofern der überlebende Ehepartner innerhalb der folgenden 20 Jahre verstirbt. 
(§ $27 \mathrm{ErbStG)}$ werden so vermieden. Eine ergänzende Härtefallregelung ist dann sinnvoll, wenn vorhandenes Gebrauchsvermögen, wie z.B. Wohneigentum, von beiden Ehepartnern gemeinsam genutzt wurde, dessen Verkauf zur Begleichung der Steuerschuld nicht zugemutet werden soll. In diesen Fällen ist eine verzinsliche Stundung der Steuerschuld möglich (die letztlich zu Lasten zukünftiger Erben geht). Alle Argumente - bis auf die letztgenannten Härtefallregelungen - liefern letztlich keine stichhaltigen Begründungen für eine Sonderbehandlung von Erbschaften und Geschenken. Es handelt sich vor allem um vorgeschobene Argumente, um vorhandene Besitzstände (hier: den nahezu steuerfreien Erbübergang) zu verteidigen ${ }^{144}$. Die Benachteiligung von auf eigener Leistung beruhenden Einkünften durch eine Sonderbehandlung von Erbschaften und Geschenken vermag in einer Leistungsgesellschaft nicht $\mathrm{zu}$ überzeugen. Zumindest eine Gleichstellung von Leistungseinkünften und Zuwendungen Dritter erscheint angemessen 145 . Voraussetzung einer steuerlichen Erfassung von Erbschaften und Geschenken ist allerdings auch hier eine Beendigung des selbstverordneten Desinteresses der Finanzbehörden gegenüber der personellen Zuordnung und zeitlichen Entwicklung (Wertveränderungen!) von privaten Vermögen.

Wenn diesbezüglich Einigkeit besteht, kann eine Integration von Erbschaften und Geschenken in die Bemessungsgrundlage der Einkommensteuer vorgenommen werden. Die Anwendung des üblichen Steuertarifs ist unter dem Aspekt der Gleichmäßigkeit der Besteuerung allerdings nur im Rahmen eines umfassenden Verfahrens der Lebenseinkommensbesteuerung unproblematisch; eine Jahresbesteuerung führt dagegen bei sog. außerordentlichen Einkünften, um die es sich hier handelt, aufgrund des progressiven Steuertarifs regelmäßig zu einer zu hohen Steuerschuld. Daher sind im Rahmen einer Jahresbesteuerung besondere Lösungen für die Behandlung außerordentlicher Einkünfte zu suchen,

144 Ein Standardbeispiel dafür bietet die Argumentation von Frank (1969, 95-97).

145 Dagegen würde durch eine Abschaffung des Erbrechts (Rüstow, 1950, 96-99) die freie Verfügung über erworbenes Eigentum in unangemessener Weise eingeschränkt. 
jedoch innerhalb des gemeinsamen Rahmens einer einheitlichen Bemessungsgrundlage und bei Verfahrensgleichheit für alle Arten außerordentlicher Einkünfte ${ }^{146}$.

Hierzu ist zunächst eine Abgrenzung des Begriffs der "außerordentlichen Einkünfte" erforderlich. Soll ein besonderes Besteuerungsverfahren nur in Ausnahmefällen zur Anwendung kommen, was sowohl aus Gründen der Verwaltungsvereinfachung als auch der Steuertransparenz sinnvoll erscheint - sonst könnte gleich das kompliziertere Lebenseinkommensbesteuerungsverfahren generell zur Anwendung kommen -, dann bietet es sich an, sowohl eine Mindesthöhe als auch die Einmaligkeit entsprechender Zuflüsse vorauszusetzen. Im Rahmen einer reinen Jahressteuer könnte die Mindesthöhe außerordentlicher Einkünfte mit beispielsweise 20.000 DM festgelegt werden, bei genereller Anwendung eines Verfahrens des interperiodischen Progressionsausgleichs deutlich höher, z.B. bei Verwendung eines 5-Jahres-Durchschnitts auf 100.000 DM.

Für das anzuwendende Verfahren sollte gelten, daß einerseits eine weitgehende Orientierung an einer Lebenseinkommensbesteuerung erfolgt, andererseits aber im Regelfall der Besteuerungsvorgang im Veranlagungsjahr der Erzielung der außerordentlichen Einkünfte abgeschlossen werden kann. Es bietet sich dann an, die jährlichen Steuerbemessungsgrundlagen der Vergangenheit um einen jeweils gleichen Anteil der außerordentlichen Einkünfte zu erhöhen. Die aktuelle Steuerschuld kann als Summe der Differenzen der Steuerbeträge bei ursprünglicher und ergänzter Steuerbemessungsgrundlage ermittelt werden. Die Bestandskraft der für die Vergangenheit erlassenen Steuerbescheide wird dadurch nicht berührt. Notwendige Informationen über die Vergangenheit sind lediglich die ursprünglichen (zu Kontrollzwecken) und indexierten Bemessungsgrundlagen der jeweiligen Jahre, die ohne großen Aufwand den jährlichen Steuerbescheiden als Anhang beigefügt werden können. Für Minderjährige kann wegen der regelmäßig nur geringen Zahl von

$146 \mathrm{Da}$ ein solcher gemeinsamer Rahmen im derzeitigen Einkommensteuergesetz schmerzlich vermißt wird, zeigt beispielsweise die gegenwärtige Behandlung von "Wiederkehrenden Bezügen" (vgl. Tipke/Lang, 1991, 360-366). 
Jahren, die in die beschriebene Durchschnittsbildung eingehen, eine Verteilung auf beispielsweise 20 Jahre durch "Gewinnvortrag" festgelegt werden. Bis ein solches Verfahren zur Anwendung kommen kann, sind im Rahmen des Einkommensteuergesetzes besondere Steuersätze für außerordentliche Einkünfte auf andere Weise zu bestimmen.

Wegen der erheblichen Differenzen zur derzeitigen steuerlichen Behandlung von Erbschaften und Geschenken ist sicherlich nur eine allmähliche Integration möglich. Im Rahmen einer separaten Erbschaftsteuer ist dazu der Übergang zu einer zeitnahen Bewertung der Vermögensübertragungen besonders dringlich (vgl. auch Wissenschaftlicher Beirat beim BMF, 1989, 14f., 31-33). Auch könnte bereits innerhalb der Erbschaftsteuer die allgemeine Differenzierung nach dem Verwandtschaftsgrad durch die Freistellung eines Vorsorgebedarfs für Hinterbliebene ersetzt werden 147 . Schließlich ist eine konsensfähige und praktikable Abgrenzung der steuerbaren Transaktionen erforderlich.

147 Damit würde der "doppelt-progressive" Erbschaftsteuertarif (Institut "Finanzen und Steuern", 1990, 5) entfallen, mit dem derzeit noch die unerbittliche Härte des Fiskus auf diesem Gebiet belegt werden kann. 


\section{ZUSAMMENFASSUNG UND AUSBLICK}

Die erarbeiteten Reformvorschläge bieten auf der Grundlage der Reinvermögenszugangstheorie für den Bereich der Transferbesteuerung ein in sich geschlossenes Gesamtkonzept und machen eine Vielzahl von inkonsistenten und intransparenten Regelungen des derzeitigen Einkommensteuerrechts überflüssig. Konzeptionell zeichnet sich die Vorgehensweise insbesondere dadurch aus, daß sie explizit eine durchgängig individualorientierte Betrachtungsweise verfolgt, die eine Voraussetzung für die umfassende Behandlung interpersonaler Transfers darstellt und gleichzeitig in intertemporaler Sicht Strukturbrüche aufgrund von Änderungen in Lebensgemeinschaften vermeidet.

In ihrer Summe bewirken die vorgeschlagenen Regelungen eine Erweiterung des derzeitigen "Markteinkommensbegriffs". Dieser läßt sich ohnehin nur dann aufrechterhalten, wenn die personelle Zuordnung von Einkommen nach anderen Kriterien erfolgt (z.B. implizit in der Ehegattenbesteuerung, explizit in $\S 22 \mathrm{Nr}$.1a EStG) und Nicht-Markteinkommen in einer Sondersteuer auf Erbschaften und Geschenke ausgegliedert werden. Gleichwohl kann ein Großteil der vorgeschlagenen Regelungen auch im Sinne der angedeuteten wenig konsequenten Interpretation einer Markteinkommenstheorie als eine Veränderung der personellen Zuordnung von Markteinkommen verstanden werden. Eine Beibehaltung der steuerlichen Erfassung von Einkünften nach Einkunftsarten ist aus erhebungstechnischen Gründen weiterhin sinnvoll.

Eine Umsetzung der vorgeschlagenen Reformen erfordert im politischen Bereich ein offensiv vertretenes Programm zu Steuergerechtigkeit und Steuervereinfachung. In einem solchen Programm muß insbesondere auch der Mut aufgebracht werden, die derzeitige selbstauferlegte Zurückhaltung der Finanzbehörden gegenüber privaten Vermögen und Vermögenseinkünften zu beenden, die von den Betroffenen bereits als sozialer Besitzstand betrachtet wird. Sind politische Mehrheiten für ein solches Programm vorhanden, dann sind längerfristig auch Lösungen für die damit implizierten sehr weitreichenden Finanzausgleichsprobleme mög- 
lich, die aufgrund von Einnahme- und Ausgabeveränderungen und -verlagerungen zwischen den Gebietskörperschaften und im Verhältnis zu den Trägern der Sozialversicherung auftreten. Das gleiche gilt für Übergangsprobleme, die besonders in den Bereichen der Alterssicherung und der Ebschaften und Schenkungen nur langfristig lösbar sind. Allzu abrupte Änderungen sind hier zu vermeiden; so ist z.B. eine pauschalierende anteilsmäßige Zuordnung von Zuflüssen zur alten Rechtslage einerseits, zur neuen Rechtslage andererseits als Übergangslösung denkbar ${ }^{1}$. Die Richtung notwendiger Reformmaßnahmen ist jedenfalls eindeutig.

Die für die Gestaltung des Einkommensteuerrechts wichtigsten Ergebnisse dieser Arbeit lassen sich - ohne auf deren Begründungslinien hier nochmals eingehen zu können - wie folgt zusammenfassen:

Von zentraler Bedeutung und daher in einem gesonderten Abschnitt des Einkommensteuergesetzes zu regeln ist die Gewährung eines steuerfreien Existenzminimums für jeden Steuerpflichtigen ("persönlicher Grundbedarf")2. Der persönliche Grundbedarf ist in Anlehnung an die Leistungen der Sozialhilfe festzulegen. Er ergibt sich nach einem "Baukastensystem" aus einer Lebensbedarfspauschale, einer Wohnungspauschale, Mehrbedarfspauschalen sowie nicht pauschalierbaren besonderen Belastungen. Die Lebensbedarfspauschale umfaßt für alle Steuerpflichtigen anfallende Aufwendungen (ohne Wohnungsaufwendungen). Für Kinder und Jugendliche ist sie nochmals nach Altersklassen zu differenzieren. Die Wohnungspauschale deckt den wohnbezogenen Bedarf ab und wird nach Regionalklassen ausgewiesen. Mehrbedarfspauschalen tragen dem besonderen Bedarf bestimmter Personengruppen Rechnung; hier ist auch ein Mehrbedarf Alleinlebender und Alleinerziehender zur Kompensation der sog. Haushaltsersparnis vorzusehen. Hinzu kommen

1 Ein solches Übergangsverfahren wird hier deshalb angedeutet, weil es einen allmählichen Übergang absichert und gleichwohl eine Automatik für die volle Umsetzung von Reformmaßnahmen beinhaltet. Der (langfristige) Gehalt von Reformmaßnahmen wird durch dieses Übergangsverfahren nicht angetastet.

2 In der Begriffswahl ergeben sich hier zum Teil Übereinstimmungen mit Lang (1985, 98-100), ohne damit jedoch gleiche Sachverhalte zu bezeichnen. 
nicht pauschalierbare Lebensbedarfsaufwendungen, soweit sie nach Art und Umfang existentiell notwendig sind. Konzeptionell gehören auch Beiträge zu Krankenversicherungen, soweit sie eine Absicherung von krankheitsbedingten Aufwendungen in angemessenem Umfang bewirken, zum steuerfreien Existenzminimum. Insbesondere wegen der Beitragsgestaltung der gesetzlichen Krankenversicherung lassen sich solche Krankenversicherungsbeiträge jedoch nicht pauschalieren und beinhalten zudem regelmäßig eine Absicherung krankheitsbedingten Ausfalls von Arbeitseinkommen - daher sind sie aus verfahrenstechnischen Gründen an anderer Stelle zu behandeln.

Der so ermittelte persönliche Grundbedarf ist um Leistungen der Sozialhilfe, Wohngeldleistungen und andere wohnungsbezogene Transfers zu kürzen, da diese dem gleichen Zweck dienen. Es ist jedoch auszuschließen, daß sich dadurch ein negativer Betrag für den persönlichen Grundbedarf ergibt (Anrechnungsverfahren).

Neben dem persönlichen Grundbedarf sind noch weitere private Abzüge zu berücksichtigen. Dies sind vor allem Unterhaltsleistungen. Als gesetzlich festgelegte Transfers können im Regelfall der Zugewinngemeinschaft (und auch im Fall der Gütergemeinschaft) Leistungen zwischen Ehepartnern pauschalierend in Höhe der Hälfte der Einkommensdifferenz angesetzt werden. Im Güterstand der Gütertrennung ist dagegen lediglich die Sicherung eines Mindestbedarfs anzuerkennen. Voraussetzung für eine hiervon abweichende einkommensteuerliche Regelung ist eine Änderung des ehelichen Güterrechts, die unter gesellschaftspolitischen Aspekten auch naheliegt (familienphasenspezifische Regelung des Güterstands). Für die einkommensteuerliche Behandlung von Unterhaltsleistungen unter Geschiedenen sollte deren gerichtlich festgesetzte Höhe maßgeblich sein. Um insbesondere auch nichtehelichen Lebensgemeinschaften eine gegenseitige Basisabsicherung zuzugestehen und weitere Unterhaltsleistungen einkommensteuerlich sinnvoll zu integrieren, sollten Transfers zur Sicherung des Existenzminimums Dritter ebenfalls von der Steuerbemessungsgrundlage des Leistenden abgezogen werden können. Eine Berücksichtigung von Unterhaltsleistungen an Kinder kann entfallen, wenn (i.d.R. den Eltern) ein allgemeines Kinder- 
geld in ausreichender Höhe gewährt wird. Zusätzlich sind bei erwerbstätigen Alleinerziehenden und Doppelverdiener-Ehepaaren Kinderbetreuungskosten in pauschaler Form zu berücksichtigen. Eine ungerechtfertigte doppelte Berücksichtigung des Grundbedarfs von Kindern wird durch die Zurechnung von Unterhaltsleistungen an Kinder ausgeschlossen. Zur Absicherung von Hinterbliebenen sind Todesfallrisikoversicherungen dann anzuerkennen, wenn sie Rentenleistungen ausschließlich für Zeiten eines besonderen Bedarfs vorsehen. Solche Zeiten sind für Ehepartner Kindererziehungszeiten und für Kinder die Zeit bis zum Abschluß einer Berufsausbildung.

Als private Einkünfte sind grundsätzlich alle Arten von leistungsfähigkeitserhöhenden Transfers zu erfassen. Dazu zählen alle empfangenen Unterhaltsleistungen (ggf. in pauschalierter Form), staatliche Transfers mit besonders geregelten Ausnahmen (wie z.B. Sozialhilfe, Wohngeld und Kindergeld), alle Versicherungsleistungen, die nicht im Rahmen anderer Einkunftsarten steuerpflichtig sind, mit Ausnahme von Sachversicherungen und der Sachleistungen bzw. Kostenerstattungen von Krankenversicherungen und schließlich sonstige laufende und einmalige Zuwendungen Dritter, insb. auch Erbschaften. Für besonders hohe einmalige Beträge sind Regelungen zur Bestimmurig des Steuersatzes anzuwenden, die allgemein für außerordentliche Einkünfte gelten. Als Werbungskosten sind im Rahmen der "privaten Einkünfte" Beiträge an reine Leibrentenversicherungen, an Unfall- und Arbeitslosenversicherungen abzugsfähig.

Sofern die Beiträge zu Krankenversicherungen nicht gesondert als Beiträge zur Sicherung krankheitsbedingten Einkommensausfalls und zur Absicherung krankheitsbedingter Kostenbelastungen ausgewiesen werden, die dann systematisch korrekt den Werbungskosten zur Erzielung privater Einkünfte bzw. dem Existenzminimum zugeordnet werden könnten, ist ihr Abzug von der Summe der Einkünfte vorzusehen. Allerdings sind Beschränkungen des Leistungskatalogs in Anlehnung an die Regelungen der gesetzlichen Krankenversicherung zu beachten.

Lebensversicherungen auf den Erlebensfall mit Ausnahme von reinen Leibrentenversicherungen sind bei steuerlicher Abzugsfähigkeit der 
Beiträge mit der Veränderung ihres Rückkaufswerts (bzw. zum tatsächlichen Auszahlungsbetrag) wie andere private Wertzuwächse zu erfassen. Beiträge zu Todesfallversicherungen sind mit Ausnahme von Rentenversicherungen für besondere Bedarfssituationen Hinterbliebener nicht von der Steuerbemessungsgrundlage absetzbar.

Die hier im Vordergrund stehenden einkommensteuerlichen Überlegungen implizieren auch Änderungen der Alterssicherungssysteme hin zu einer voll eigenständigen Sicherung von Ehepartnern (nach dem Grundprinzip des Splitting von Ansprüchen unter Einbezug von Kindererziehungszeiten). Voraussetzungen hierfür sind die Abschaffung im allgemeinen Beitrag enthaltener (bedarfsunabhängiger ${ }^{3}$ ) Hinterbliebenensicherungsleistungen und eine mit dem jeweiligen Anspruchszeitraum abschließend vorgenommene Bewertung von Anspruchszeiten.

Ein allgemeines Kindergeld in ausreichender Höhe wird am besten der Interdependenz von steuerlichen und sozialpolitischen Anforderungen an den Kinderlastenausgleich gerecht. Es ersetzt eine steuerliche Berücksichtigung von Unterhaltsverpflichtungen für Kinder. Gleichzeitig wird vermieden, daß die Unterhaltsverpflichtungen für Kinder Sozialhilfebedürftigkeit der Eltern verursachen.

Förderungstatbestände innerhalb des Einkommensteuerrechts sind in einem gesonderten Abschnitt aufzuführen. Die separate Behandlung weist sie als steuersystematisch nicht zu vertretende besondere Normen aus, was der Transparenz des Steuersystems dient und gleichzeitig einer laufenden Überprüfung der Angemessenheit dieser Sonderregelungen förderlich ist. Hierher gehören auch Bestimmungen zur Steuerfreiheit bestimmter staatlicher Transfers.

Durch eine stärker individualorientierte Zurechnung steuerlicher Leistungsfähigkeit wird auch das Erhebungsverfahren der Einkommensteuer berührt. Für Ehepartner und deren minderjährige Kinder kann gleichwohl auch eine prinzipiell auf individuellen Bemessungsgrundlagen aufbauende Veranlagung wie bisher in einem vereinfachten gemeinsamen

3 Die Finanzierung einer bedarfsabhängigen Hinterbliebenensicherung aus allgemeinen Haushaltsmitteln oder auch durch die Solidargemeinschaft der Versicherten läßt sich meines Erachtens vertreten. 
Verfahren erfolgen. Dies sollte einhergehen mit einer Neugliederung und Vereinfachung des Einkommensteuerrechts insgesamt, einer übersichtlichen und verständlichen Gestaltung von Steuerformularen und -bescheiden sowie einer Aufstockung des Personals der Finanzämter. Damit kann dann nicht nur eine größere Steuergerechtigkeit und Steuertransparenz erreicht werden, sondern auch eine Erhöhung des Interesses und Informationsstandes der betroffenen Bürger, was in einer demokratischen Gesellschaft für die Einkommensteuer als wichtigstes staatliches Finanzierungsinstrument unerläßlich ist ${ }^{4}$.

Die Abschaffung unterschiedlicher Lohnsteuerklassen für Ehepaare ist unter Gleichbehandlungsaspekten geboten und stellt eine systemgerechte Antwort auf den "Zweitverdienereffekt" dar.

Eine Bündelung der finanziellen Beziehungen zwischen Bürger und Staat bei den Finanzämtern läßt sich durch eine Übertragung der Zuständigkeiten für Kindergeld und Wohngeld erreichen. Damit sind Vereinfachungseffekte für Antragsteller und Kosteneinsparungen in der Verwaltung möglich. Fiktive Steuerveranlagungen von Sozialhilfeempfängern liefern die Grundlage für eine bessere Abstimmung der Existenzminima im Sozialhilfe- und Einkommensteuerrecht.

4 Dazu paßt auch die Einführung einer obligatorischen Steuerveranlagung (bzw. eines Lohnsteuerjahresausgleichs) für alle Steuerpflichtigen (Mitschke, 1985, 69). 


\section{LITERATURVERZEICHNIS}

Achinger, Hans / Höffner, Joseph / Muthesius, Hans / Neundörfer, Ludwig, 1955, Neuordnung der sozialen Leistungen, Köln.

Albers, Willi, 1957, Die Einkommensbesteuerung in Frankreich seit dem Ersten Weltkrieg. Eine Analyse ihrer wirtschaftlichen, sozialen und administrativen Probleme, Kiel.

Albers, Willi, 1967a, Zur Reform des Familienlastenausgleichs in der Bundesrepublik Deutschland, Sozialer Fortschritt, 16, 199-204.

Albers, Willi, 1967b, Probleme eines Familienlastenausgleichs, Sozialer Fortschritt, 16, 158-162.

Albers, Willi, 1977, Transferzahlungen an Haushalte, in: Fritz Neumark, Norbert Andel und Heinz Haller (Hrsg.), Handbuch der Finanzwissenschaft, 3.Aufl., Bd.1, Tübingen, 861-957.

Albers, Willi, 1980, Einkommensbesteuerung I: Einkommensteuer, in: Willi Albers u.a. (Hrsg.), Handwörterbuch der Wirtschaftswissenschaft, Bd.2, Stuttgart u.a.O., 189-218.

Albers, Willi, 1988, Das Splittingverfahren in der Einkommensteuer, Wirtschaftsdienst, $68,412-418$.

Albers, Willi, 1989, Grundsatzfragen der Familienpolitik, Wirtschaftsdienst, 69, 338-344.

Altfelder, Stefan, 1987, Steuerliche Gestaltung des Ehegatten- und Kindesunterhalts, Köln u.a.O.

Andel, Norbert, 1970, Die einkommensteuerliche Behandlung der Beiträge an und der Leistungen von Altersversicherungen, in: Heinz Haller u.a. (Hrsg.), Theorie und Praxis des finanzpolitischen Interventionismus, Fritz Neumark zum 70. Geburtstag, Tübingen, 327-344.

Andel, Norbert, 1977, Subventionen, in: Willi Albers u.a. (Hrsg.), Handwörterbuch der Wirtschaftswissenschaft, Bd.7, Stuttgart u.a.O., 491-510.

Andel, Norbert, 1979, Nettoanpassung und Besteuerung der Renten im Lichte der Gleichmäßigkeit der Besteuerung, der Verteilungsgerechtigkeit und des Sanierungsbedarfs der Rentenversicherungen, in: Peter Bohley und Georg Tolkemitt (Hrsg.), Wirtschaftswissenschaft als Grundlage staatlichen Handelns, Heinz Haller zum 65. Geburtstag, Tübingen, 165-176.

Andel, Norbert, 1980, Einkommensteuer, in: Fritz Neumark, Norbert Andel und Heinz Haller (Hrsg.), Handbuch der Finanzwissenschaft, 3.Aufl., Bd.2, Tübingen, 331-401.

Andel, Norbert, 1984, Zum Konzept der meritorischen Güter, Finanzarchiv, N.F.42, 630-648. 
Andel, Norbert, 1990, Finanzwissenschaft, 2.Aufl., Tübingen.

Andrews, William D., 1972/73, Personal Deductions in an Ideal Income Tax, Harvard Law Review, 86, 309-385.

Angermann, Oswald, 1989, Vergleich der Verbraucherpreisniveaus in Bonn, Karlsruhe, München und Berlin (West), Wirtschaft und Statistik, 258261.

Arbeitsgemeinschaft "Riedmüller / Glatzer / Infratest", 1991, Die Lebenssituation alleinstehender Frauen, Schriftenreihe des Bundesministers für Frauen und Jugend, Bd.1, Stuttgart - Berlin - Köln.

Arrow, Kenneth J., 1973, Some Ordinalist-Utilitarian Notes on Rawls's Theory of Justice, Journal of Philosophy, 70, 245-263.

Atkinson, Anthony Barnes, 1989, Poverty and Social Security, New York u.a.O.

Auge, Michael, 1984, Humanvermögen, Sozialisation und Familienlastenausgleich. Zur vermögenstheoretischen Perspektive in der Familienpolitik, Spardorf.

Aumüller, Günther / Balzer, Albrecht, 1991, Tarife und Bedingungen der privaten Krankenversicherung, Loseblattausgabe, 29.Folge, Karlsruhe.

Bachmann, Ernst-Udo, 1982, Kindergeld und Steuerrecht, Deutsche SteuerZeitung, 70, 318-322.

Biergans, Enno / Stockinger, Roland, 1982, Zum Einkommensbegriff und zur persönlichen Zurechnung von Einkünften im Einkommensteuerrecht, Finanz-Rundschau, 37, 1-7, 25-33.

Biergans, Enno / Wasmer, Claudius, 1985, Zum Tatbestand der Besteuerung und zum Leistungsfähigkeitsbegriff in der Einkommensteuer, FinanzRundschau, 40, 57-63.

Birk, Dieter, 1983, Das Leistungsfähigkeitsprinzip als Maßstab der Steuernormen, Köln.

Birk, Dieter, 1986a, unter Mitarbeit von Jutta Förster, Notwendigkeit und Grenzen einer Neuregelung einer Besteuerung von Renten aus verfassungsrechtlicher Sicht, Gutachten erstattet im Auftrag des Verbandes Deutscher Rentenversicherungsträger, Frankfurt.

Birk, Dieter, 1986b, Steuergerechtigkeit und Rentenbesteuerung - Überlegungen zu verfassungsrechtlichen Grundfragen der Neuordnung der Rentenbesteuerung, Deutsche Rentenversicherung, 129-142.

Birtel, Thomas, 1985, Die Zeit im Einkommensteuerrecht, Berlin.

Bittker, Boris I., 1968, A "Comprehensive Tax Base" as a Goal of Income Tax Reform, in: Boris I. Bittker u.a., A Comprehensive Income Tax Base? A Debate, Branford, 1-61.

Borth, Helmut, 1989, Ehegattenunterhalt, in: Dieter Schwab (Hrsg.), Handbuch des Scheidungsrechts, 2.Aufl., München, 549-863. 
Boulding, Kenneth E., 1973, The Economy of Love and Fear, Belmont.

Boulding, Kenneth E. / Pfaff, Martin, 1972, Transferwirtschaft: Regelungstheorie der Wirtschafts- und Sozialpolitik, Wirtschaftswoche, 26, Nr.36 vom 8.9.1972, 35-42.

Brandstätter, Hermann / Kirchler, Erich / Wagner, Wolfgang, 1987, Rücksichtnahme und Betroffenheit von Ehepartnern in Konfliktsituationen, in: Horst Todt (Hrsg.), Die Familie als Gegenstand sozialwissenschaftlicher Forschung, Schriften des Vereins für Socialpolitik, N.F.164, Berlin, 147161.

Brazer, Harvey E., 1980, Income Tax Treatment of the Family, in: Henry J. Aaron und Michael J. Boskin (Hrsg.), The Economics of Taxation, Washington, 223-246.

Brennan, Geoffrey, 1971, Horizontal Equity: An Extension of an Extension, Public Finance, 26, 437-456.

Brennan, Geoffrey / Buchanan, James M., 1980, The Power to Tax. Analytical Foundations of a Fiscal Constitution, Cambridge u.a.O.

Bretz, Manfred / Niemeyer, Frank, 1992, Private Haushalte gestern und heute, Wirtschaft und Statistik, 73-81.

Browning, Edgar K., 1975, The Externality Argument for In-Kind Transfers: Some Critical Remarks, Kyklos, 28, 526-544.

Brox, Hans, 1991, Erbrecht, 13.Aufl., Köln u.a.O.

Brühl, Albrecht, 1989, Mein Recht auf Sozialhilfe, 6.Aufl., München.

Brümmerhoff, Dieter, 1973/74, Nominal- oder Realprinzip in der Einkommensbesteuerung?, Finanzarchiv, N.F.32, 35-45.

Brümmerhoff, Dieter, 1979, Die Einkommensbesteuerung der Sozialversicherungsrenten, Steuer und Wirtschaft, 56, 219-227.

Brümmerhoff, Dieter, 1990, Finanzwissenschaft, 5.Aufl., München - Wien.

Buchanan, James M., 1967, Public Finance in Democratic Process, Chapel Hill.

Budd, Edward C. / Radner, Daniel B. / Whiteman, T. Cameron, 1984, An Accounting Framework for Transfer Payments and Its Implications for the Size Distribution of Income, in: Marilyn Moon (Hrsg.), Economic Transfers in the United States, Chicago - London, 37-86.

Bundesfinanzhof, 1979, Urteil vom 8. März 1979 (IV R 94/75), Bundessteuerblatt II, 29, 410-412.

Bundesfinanzhof, 1986, Urteil vom 29. Juli 1986 (IX R 206/84), Bundessteuerblatt II, 36, 747-749.

Bundesminister der Finanzen, 1990, Verzinsung von Steuernachforderungen und Steuererstattungen ( $\$ 233 \mathrm{a} \mathrm{AO}$ ), Schreiben vom 4.7.1990, Bundessteuerblatt I, 304-309. 
Bundesminister für Arbeit und Sozialordnung (Hrsg.), 1990, Sozialbericht 1990, Bonn.

Bundesminister für Bildung und Wissenschaft (Hrsg.), 1992, BAföG '92/93. Gesetz und Beispiele, Bonn.

Bundesminister für Jugend, Familie, Frauen und Gesundheit (Hrsg.), 1986, Vierter Familienbericht. Die Situation der älteren Menschen in der Familie, Bonn (gleichzeitig Bundestags-Drucksache 10/6145).

Bundesministerium der Finanzen (Hrsg.), 1987, Bericht der Bundesregierung über die Entwicklung der Finanzhilfen des Bundes und der Steuervergünstigungen für die Jahre 1985 bis 1988 (Elfter Subventionsbericht), Bonn.

Bundesministerium der Finanzen (Hrsg.), 1991, Bericht der Bundesregierung über die Entwicklung der Finanzhilfen des Bundes und der Steuervergünstigungen für die Jahre 1989 bis 1992 (Dreizehnter Subventionsbericht), Bonn.

Bundesverfassungsgericht, 1957, Urteil vom 17.1.1957, in: Dass., Entscheidungen des Bundesverfassungsgerichts, Bd.6, Tübingen, 55-84.

Bundesverfassungsgericht, 1959, Urteil vom 14.4.1959, in: Dass., Entscheidungen des Bundesverfassungsgerichts, Bd.9, Tübingen, 237-250.

Bundesverfassungsgericht, 1965, Urteil vom 30.6.1964, in: Dass., Entscheidungen des Bundesverfassungsgerichts, Bd.18, Tübingen, 97-112.

Bundesverfassungsgericht, 1977, Urteil vom 23.11.1976, in: Dass., Entscheidungen des Bundesverfassungsgerichts, Bd.43, Tübingen, 108-125.

Bundesverfassungsgericht, 1980, Urteil vom 28.2.1980, in: Dass., Entscheidungen des Bundesverfassungsgerichts, Bd.53, Tübingen, 257-313.

Bundesverfassungsgericht, 1983, Urteil vom 3.11.1982, in: Dass., Entscheidungen des Bundesverfassungsgerichts, Bd.61, Tübingen, 319-357.

Bundesverfassungsgericht, 1984, Urteil vom 22.2.1984, in: Dass., Entscheidungen des Bundesverfassungsgerichts, Bd.66, Tübingen, 214-226.

Bundesverfassungsgericht, 1985a, Urteil vom 17.10.1984, in: Dass., Entscheidungen des Bundesverfassungsgerichts, Bd.68, Tübingen, 143-155.

Bundesverfassungsgericht, 1985b, Urteil vom 16.7.1985, in: Dass., Entscheidungen des Bundesverfassungsgerichts, Bd.69, Tübingen, 272-315.

Bundesverfassungsgericht, 1991a, Urteil vom 29.5.1990, in: Dass., Entscheidungen des Bundesverfassungsgerichts, Bd.82, Tübingen, 60-105.

Bundesverfassungsgericht, 1991b, Urteil vom 12.6.1990, in: Dass., Entscheidungen des Bundesverfassungsgerichts, Bd.82, Tübingen, 198-208.

Busch, Roswitha, 1975, Steuerentlastungen oder Transferzahlungen, Köln.

Clotfelder, Charles T., 1985, Federal Tax Policy and Charitable Giving, Chicago - London.

Deininger, Dieter, 1991, Sozialhilfeempfänger 1989, Wirtschaft und Statistik, $457-465$. 
Denison, Edward F., 1976, Price Series for Indexing the Income Tax System, in: Henry J. Aaron (Hrsg.), Inflation and the Income Tax, Washington, 233-261.

Deutscher Bundestag, 1974, Entwurf eines Dritten Steuerreformgesetzes (Gesetzentwurf der Bundesregierung), Bundestags-Drucksache 7/1470 vom 9.1.1974.

Deutscher Juristentag, 1974, Verhandlungen des 50. Deutschen Juristentags, München.

Deutscher Juristentag, 1988, Verhandlungen des 57. Deutschen Juristentags, München.

Domar, Evsey D. / Musgrave, Richard A., 1959, Proportional Income Taxation and Risk-Taking, in: American Economic Association (Hrsg.), Readings in the Economics of Taxation, London, 493-524.

Dornbusch, Hans-Ludwig, 1982, Ein Vorschlag zur Neugestaltung des Kinderlastenausgleichs, Wirtschaftsdienst, 62, 544-548.

Dziadkowski, Dieter, 1986, Grundfreibetrag und Einkommensteuertarif, Finanz-Rundschau, 68, 504-509.

Ebnet, Otto, 1978, Die Besteuerung des Wertzuwachses, Baden-Baden.

Eisen, Roland, 1988, "Versicherungsprinzip" und Umverteilung - Einige theoretische Überlegungen zu den Grenzen des Versicherbaren, in: Gabriele Rolf, P. Bernd Spahn und Gert Wagner (Hrsg.), Sozialvertrag und Sicherung. Zur ökonomischen Theorie staatlicher Versicherungs- und Umverteilungssysteme, Frankfurt - New York, 117-127.

Erbe, Rainer, 1986, Familienlastenausgleich über die gesetzliche Rentenversicherung?, Wirtschaftsdienst, 66, 194-202.

Feldstein, Martin, 1976, On the Theory of Tax Reform, Journal of Public Economics, 6, 77-104.

Frank, Dieter, 1969, Erbschaftsteuer und Unternehmung. Eine steuerrechtliche und betriebswirtschaftliche Untersuchung unter Berücksichtigung des britischen, französischen und schweizerischen Rechts, Berlin.

Fuisting, B., 1899, Die Preußischen direkten Steuern, Bd.1: Kommentar zum Einkommensteuergesetz, 4.Aufl., Berlin.

Gaddum, Johann Wilhelm, 1986, Steuerreform: Einfach und gerecht!, Stuttgart.

Gaertner, Wulf, 1988, Untergrundwirtschaft, Steuerhinterziehung und Moral, in: Helmut Hesse (Hrsg.), Wirtschaftswissenschaft und Ethik, Berlin, 109-130.

Galler, Heinz P. / Ott, Notburga, 1987, Familienlastenausgleich: effizientere Lösungen sind möglich, Wirtschaftsdienst, 67, 402-408.

Gandenberger, Otto, 1989, Einkommensabhängige staatliche Transfers, BadenBaden. 
Geiger, Peter, 1987, Und nochmals: Rentenversicherungsbeiträge eines Arbeitnehmers als vorab entstandene Werbungskosten bei den sonstigen Einkünften?, Finanz-Rundschau, 42, 468-469.

Gernhuber, Joachim, 1980, Lehrbuch des Familienrechts, 3.Aufl., München.

Giloy, Jörg, 1978, Vieldeutige Einkommensbegriffe. Zur geeigneten Bezugsgröße staatlicher Transferleistungen, Herne - Berlin.

Giloy, Jörg, 1979a, Zur Periodizität der Einkommensteuer, Finanz-Rundschau, 34, 133-137.

Giloy, Jörg, 1979b, Zur Abzugsfähigkeit vorweggenommener Werbungskosten bei Kapitaleinkünften, Betriebs-Berater, 34, 1708-1710.

Giloy, Jörg, 1982, Zur Besteuerung von Staatsleistungen, Finanz-Rundschau, 37, 129-130.

Goode, Richard, 1987, Disappointed Expectations and Tax Reform, National Tax Journal, 40, 159-169.

Gordon, David M., 1972, Taxation of the Poor and the Normative Theory of Tax Incidence, American Economic Review, 62, Papers and Proceedings, 319-328.

Graetz, Michael J., 1980, Expenditure Tax Design, in: Joseph A. Pechman (Hrsg.), What Should Be Taxed: Income or Expenditure?, Washington, 161-276.

Groß, Jürgen, 1988, "Die Besteuerung der Renten" - unter besonderer Berücksichtigung einer Reform des Systems der sozialen Sicherung, Diss. Kassel.

Grünärml, F. / Henke, K.-D., 1973/74, Grants Economics: Ansatzpunkt für eine Theorie der "Nicht-Markt-Ströme"?, Jahrbücher für Nationalökonomie und Statistik, 188, 236-255.

Hackmann, Johannes, 1972/73, Ein Vorschlag zur Ehegattenbesteuerung, Finanzarchiv, N.F.31, 495-518.

Hackmann, Johannes, 1979, Die Besteuerung des Lebenseinkommens, Tübingen.

Hackmann, Johannes, 1983, Die Bestimmung des steuerrechtlichen Einkommensbegriffs aus finanzwissenschaftlicher Sicht, in: K.-H. Hansmeyer (Hrsg.), Staatsfinanzierung im Wandel, Schriften des Vereins für Socialpolitik, N.F.134, Berlin, 661-702.

Hagelschuer, Paul, 1983, Lebensversicherung, Wiesbaden 1983.

Haig, Murray, 1959, The Concept of Income - Economic and Legal Aspects, in: American Economic Association (Hrsg.), Readings in the Economics of Taxation, London, 54-76. 
Haller, Heinz, 1973, Die Berücksichtigung des Lebensunterhalts der Kinder und der Vorsorgeaufwendungen im Rahmen der Steuerreform - Zielsetzungen und Implikationen, Zeitschrift für die gesamte Staatswissenschaft, 129, 504-534.

Haller, Heinz, 1981a, Die Steuern. Grundlinien eines rationalen Systems öffentlicher Abgaben, 3.Aufl., Tübingen.

Haller, Heinz, 1981b, Besteuerung der Familieneinkommen und Familienlastenausgleich, Tübingen.

Hansmeyer, Karl-Heinrich, 1963, Effekte der Einkommensumverteilung in der sozialen Krankenversicherung, in: Wilfrid Schreiber (Hrsg.), Gesetzliche Krankenversicherung in einer freiheitlichen Gesellschaft, Berlin, 105112.

Harberger, Arnold C., 1974, Taxation, Resource Allocation and Welfare, in: The Role of Direct and Indirect Taxes in the Federal Reserve System, Princeton 1964, wiederabgedruckt in: Arnold C. Harberger, Taxation and Welfare, Chicago - London, 25-62.

Head, John G., 1966, On Merit Goods, Finanzarchiv, N.F.25, 1-29.

Head, John G., 1982, The Comprehensive Tax Base Revisited, Finanzarchiv, N.F.40, 193-210.

Heldmann, Elanie, 1986, Kinderlastenausgleich in der Bundesrepublik Deutschland. Darstellung, kritische Würdigung und Analyse alternativer Reformmöglichkeiten, Frankfurt - New York.

Henke, Klaus-Dirk, 1987, Steuerliche Behandlung der Vorsorgeaufwendungen und der Alterseinkünfte aus finanzwissenschaftlicher Sicht, in: Gesellschaft für Versicherungswissenschaft und -gestaltung (Hrsg.), Steuerliche Behandlung der Alterseinkünfte, Köln, 28-41.

Henke, Klaus-Dirk, 1988, Die betriebliche Altersversorgung aus einkommensteuersystematischer Sicht, Finanzarchiv, N.F.46, 268-282.

Herrmann / Heuer / Raupach, 1974, Kommentierung von § 26 EStG, in: Dies., Einkommensteuer- und Körperschaftsteuergesetz. Kommentar, Lieferung 110.

Herrmann / Heuer / Raupach, 1976, Kommentierung von § 1 EStG, in: Dies., Einkommensteuer- und Körperschaftsteuergesetz. Kommentar, Lieferung 116.

Hettich, Walter, 1979, Henry Simons on Taxation and the Economic System, National Tax Journal, 32, 1-9.

Hettich, Walter, 1983, Reforms of the Tax Base and Horizontal Equity, National Tax Journal, 36, 417-427.

Heuer, Gerhard, 1987, Kommentierung von $\S 10$ EStG, in: Herrmann / Heuer / Raupach, Einkommensteuer- und Körperschaftsteuergesetz. Kommentar, Lieferung 150.

Hicks, J.R., 1946, Value and Capital, 2.Aufl., Oxford. 
Hinterberger, Friedrich / Müller, Klaus / Petersen, Hans-Georg, 1987, "Gerechte" Tariftypen bei alternativen Opfertheorien und Nutzenfunktionen, Finanzarchiv, N.F.45, 45-69.

Holzmann, Robert, 1984, Lebenseinkommen und Verteilungsanalyse, Berlin u.a.O.

Hotelling, Harold, 1969, The General Welfare in Relation to Problems of Taxation and of Railway and Utility Rates, in: Kenneth J. Arrow und Tibor Scitovsky (Hrsg.), Readings in Welfare Economics, London, 284308.

Huber, Bernd, 1991/92, Besteuerung, intertemporale Neutralität und zeitliche Inkonsistenz, Finanzarchiv, N.F.49, 423-456.

Infratest Sozialforschung, 1990, Alterssicherung in Deutschland 1986, Forschungsprojekt im Auftrag des Bundesministers für Arbeit und Sozialordnung, München.

Institut "Finanzen und Steuern", 1985, Einkommensbegriffe und Einkommensermittlung in den Transfergesetzen - Analyse, Kritik, Änderungsvorschläge -, Bonn.

Institut "Finanzen und Steuern", 1990, Die zunehmende Belastung der mittelständischen Unternehmen mit Erbschaftsteuer - Vorschläge zur Änderung des Erbschaftsteuergesetzes, Bonn.

Institut für Familienrecht, 1992, Düsseldorfer Tabelle (Stand 1.Juli 1992), Zeitschrift für das gesamte Familienrecht, 39, 398-400.

Jakob, Wolfgang / Jüptner, Roland, 1983, Zur Zwangsläufigkeit außergewöhnlicher Belastungen, Steuer und Wirtschaft, 60, 206-216.

Jecht, Horst, 1955/56, Die Bundessteuerreform 1954. Kritischer Rückblick auf ihre wichtigsten Ergebnisse, Finanzarchiv, N.F.16, 31-53.

Kaldor, Nicholas, 1955, An Expenditure Tax, London.

Kaltenbach, Helmut, 1987, Eigentumsschutz des Grundgesetzes in der Rentenversicherung - Möglichkeiten und Grenzen des Gesetzgebers -, in: Reinhold Schulze u.a., 14. Presseseminar der BfA Berlin - September 1987, Berlin, 51-80.

Kalthoener, Elmar / Büttner, Helmut, 1989, Die Rechtsprechung zur Höhe des Unterhalts, 4.Aufl., München.

Kalwar, Hans, 1988, Steuern und Versicherungsnehmer, in: Dieter Farny u.a. (Hrsg.), Handwörterbuch der Versicherung, Karlsruhe, 827-833.

Kammann, H. Werner, 1980, Umverteilungswirkungen und zeitliche Entwicklung des Sozialtransfer-Systems, Frankfurt/Main.

Kanzler, Hans-Joachim, 1986, Kinderbetreuungskosten als außergewöhnliche Belastung, Finanz-Rundschau, 41, 1-9.

Kanzler, Hans-Joachim, 1988, Familienarbeit, Erziehungs- und Pflegeleistung im Steuerrecht, Finanz-Rundschau, 70, 205-219. 
Karl-Bräuer-Institut des Bundes der Steuerzahler, 1971, Der Weg zu einem zeitgemäßen Steuersystem. Diskussionsbeiträge zur Reform des materiellen Steuerrechts, Wiesbaden.

Karl-Bräuer-Institut des Bundes der Steuerzahler, 1989, Kinderfreibetrag und Grundgesetz, Wiesbaden.

Karl-Bräuer-Institut des Bundes der Steuerzahler, 1991, Steuerbefreiung für das Existenzminimum!, Wiesbaden.

Kassella, Thomas / Spahn, P. Bernd, 1990, Zur Reform der Familienbesteuerung: Die steuerliche Förderung von Ehe und Familie unter Verteilungsaspekten, Sonderforschungsbereich 3 der Johann Wolfgang GoetheUniversität Frankfurt und der Universität Mannheim, Arbeitspapier Nr. 314, Frankfurt/Main - Mannheim.

Kausemann, E.-Peter, 1983, Möglichkeiten einer Integration von Steuer- und Transfersystem, Thun - Frankfurt/Main.

Kirchhof, Paul, 1988, Empfiehlt es sich, das Einkommensteuerrecht zur Beseitigung von Ungleichbehandlungen und zur Vereinfachung neu zu ordnen?, in: Verhandlungen des 57. Deutschen Juristentages, München, F1-F96.

Kisker, Klaus Peter, 1964, Die Erbschaftsteuer als Mittel der Vermögensredistribution. Eine empirische und theoretische Untersuchung, Berlin.

Klanberg, Frank, 1984, Soziale Sicherheit als System von Property Rights: Das Beispiel des Generationenvertrages, in: Manfred Neumann (Hrsg.), Ansprüche, Eigentums- und Verfügungsrechte, Berlin, 417-434.

Klein, Thomas, 1986, Äquivalenzskalen - Ein Literatursurvey, Sonderforschungsbereich 3 der Johann Wolfgang Goethe-Universität Frankfurt und der Universität Mannheim, Arbeitspapier Nr. 195, Frankfurt/Main Mannheim.

Knorr-Anders, Esther, 1988, "Am besten wär's, euch zeitig totzuschlagen", DIE ZEIT, Nr.1, 13.

Koch, Woldemar, 1966, Eine neue Deutung der Steuergrundsätze, Finanzarchiv, N.F.25, 316-321.

Köhler, Wolfgang, 1987, Handbuch des Unterhaltsrechts, 7.Aufl., München.

Kolb, Rudolf, 1985, Die Bedeutung des Versicherungsprinzips für die gesetzliche Rentenversicherung, in: Winfried Schmähl (Hrsg.), Versicherungsprinzip und soziale Sicherung, Tübingen, 120-140.

Krause, Peter, 1982, Eigentum an subjektiven öffentlichen Rechten, Berlin.

Kreßmann, Karl, 1971, Das versicherungstechnische Äquivalenzprinzip in der gesetzlichen Altersversicherung der Bundesrepublik Deutschland, Diss., Frankfurt/Main. 
Krupp, Hans-Jürgen, 1978, Das monetäre Transfersystem in der Bundesrepublik Deutschland - Elemente einer Gesamtbilanz, in: Hans-Jürgen Krupp und Wolfgang Glatzer (Hrsg.), Umverteilung im Sozialstaat, Frankfurt/Main - New York, 21-69.

Krupp, Hans-Jürgen, 1987, Perspektiven einer Strukturreform der sozialen Alterssicherung, Aus Politik und Zeitgeschichte, Beilage zur Wochenzeitung "Das Parlament", B35/87 vom 29.8.1987, 3-16.

Krupp, Hans-Jürgen, 1988, Zur Begründung und zur Praxis des Wohlfahrtsstaates, in: Gabriele Rolf, P. Bernd Spahn und Gert Wagner (Hrsg.), Sozialvertrag und Sicherung. Zur ökonomischen Theorie staatlicher Versicherungs- und Umverteilungssysteme, Frankfurt/Main - New York, 491-511.

Kullmer, Lore, 1960, Die Ehegattenbesteuerung. Ihre Geschichte, Problematik und Neuregelung in Deutschland, diskutiert unter Berücksichtigung der Erfahrungen in den USA, Frankfurt/Main.

Lamers, Bernhard, 1975, Intertemporale Einkommenstransfers - Probleme ihrer einkommensteuerlichen Behandlung und ihre Begünstigung durch Prämien in der Bundesrepublik Deutschland, Diss., Münster.

Lampe, Adolf, 1928, Die wirtschaftlichen Voraussetzungen der nichtfiskalischen Steuergestaltung, in: Hans Teschemacher (Hrsg.), Beiträge zur Finanzwissenschaft, Festgabe für Georg von Schanz zum 75. Geburtstag, Tübingen, Bd.I, 172-210.

Lampert, Heinz, 1991, Lehrbuch der Sozialpolitik, 2.Aufl., Berlin u.a.O.

Lang, Joachim, 1983, Familienbesteuerung, Steuer und Wirtschaft, 60, 103125.

Lang, Joachim, 1984, Zur Reform der Familienbesteuerung, Steuer und Wirtschaft, 61, 129-132.

Lang, Joachim, 1985, Reformentwurf zu Grundvorschriften des Einkommensteuergesetzes, Köln.

Lang, Joachim, 1987, Die einfache und gerechte Einkommensteuer. Ziele, Chancen und Aufgaben einer Fundamentalreform, Köln.

Lang, Joachim, 1988, Die Bemessungsgrundlage der Einkommensteuer. Rechtssystematische Grundlagen steuerlicher Leistungsfähigkeit im deutschen Einkommensteuerrecht, Habil. Köln 1981, Köln.

Lang, Joachim, 1990, Verfassungsrechtliche Gewährleistung des Familienexistenzminimums im Steuer- und Kindergeldrecht. $\mathrm{Zu}$ den Beschlüssen des Bundesverfassungsgerichts vom 29.5.1990 und vom 12.6.1990, Steuer und Wirtschaft, 67, 331-348.

Laum, Bernhard, 1960, Schenkende Wirtschaft. Nichtmarktmäßiger Güterverkehr und seine soziale Funktion, Frankfurt/Main.

Lehmann, Matthias, 1975, Zur Theorie der Zeitpräferenz. Ein Beitrag zur mikroökonomischen Kapitaltheorie, Diss. Frankfurt/Main 1973, Berlin. 
Leibfritz, Willi / Parsche, Rüdiger, 1988, Umverteilung in der Bundesrepublik Deutschland. Das Zusammenwirken von Steuern und Sozialtransfers, Bd.I: Status quo und Reformalternativen, München.

Lewis, Alan, 1982, The Psychology of Taxation, New York.

Littmann, Konrad, 1968, Kritische Marginalien zur Kontroverse "Individuelle Veranlagung oder Haushaltsbesteuerung", Finanzarchiv, N.F.27, 174186.

Littmann, Konrad, 1970, Ein Valet dem Leistungsfähigkeitsprinzip, in: Heinz Haller u.a. (Hrsg.), Theorie und Praxis des finanzpolitischen Interventionismus, Fritz Neumark zum 70. Geburtstag, Tübingen, 113-134.

Littmann, Konrad, 1983, Besteuerung von Alterseinkommen, in: Sachverständigenkommission Alterssicherungssysteme, Gutachten vom 19. November 1983, Berichtsband 2: Darstellung der Alterssicherungssysteme und der Besteuerung von Alterseinkommen, Stuttgart u.a.O., 425-518.

Lukarsch, Gerhard, 1988, Versicherung als Wirtschaftsgut, in: Dieter Farny u.a. (Hrsg.), Handwörterbuch der Versicherung, Karlsruhe, 959-964.

Mackscheidt, Klaus, 1976, Öffentliche Güter und Ausgabeninzidenz, in: Clemens-August Andreae, Klaus Mackscheidt und Horst Zimmermann, Öffentliche Finanzwirtschaft und Verteilung IV, hrsg. von Wilhelmine Dreißig, Berlin, 59-129.

Mackscheidt, Klaus, 1983, Transfers, Das Wirtschaftsstudium, 41-46, 87-91.

Mackscheidt, Klaus, 1986, Probleme des Bundeszuschusses, in: Gesellschaft für Versicherungswissenschaft und -gestaltung (Hrsg.), Wissenschaftler zur Strukturreform der Rentenversicherung, Bergisch-Gladbach, 18-33.

Mahr, Werner, 1951, Einführung in die Versicherungswirtschaft, Berlin.

Maydell, Bernd von, 1983, Betriebliche Altersversorgung, in: Sachverständigenkommission Alterssicherungssysteme, Gutachten vom 19. November 1983, Berichtsband 2: Darstellung der Alterssicherungssysteme und der Besteuerung von Alterseinkommen, Stuttgart u.a.O., 243-281.

Meade, J. E., 1978, The Structure and Reform of Direct Taxation, Report of a Committee Chaired by Professor J. E. Meade, London - Boston Sydney.

Meinhold, Helmut, 1985, Die ordnungspolitische Bedeutung des Versicherungsprinzips in der deutschen Sozialpolitik, in: Winfried Schmähl (Hrsg.), Versicherungsprinzip und soziale Sicherung, Tübingen, 13-26.

Mennel, Annemarie, 1974, Teilgutachten Steuerrecht, in: Manfred Löwisch, Wolfgang Gitter und Annemarie Mennel, Welche rechtlichen Maßnahmen sind vordringlich, um die tatsächliche Gleichstellung der Frauen mit den Männern im Arbeitsleben zu gewährleisten?, Gutachten für den 50. Deutschen Juristentag, in: Deutscher Juristentag, Verhandlungen des 50. Deutschen Juristentags, München, D163-D203. 
Mennel, Annemarie, 1988, Frauen, Steuern, Staatsausgaben. Subventionen für das Patriarchat, in: Ute Gerhard, Alice Schwarzer und Vera Slupik (Hrsg.), Auf Kosten der Frauen. Frauenrechte im Sozialstaat, Weinheim - Basel.

Metze, Ingolf, 1982, Negative Einkommensteuer, in: Willi Albers u.a. (Hrsg.), Handwörterbuch der Wirtschaftswissenschaft, Bd.9, Stuttgart u.a.O., 788-799.

Mill, John Stuart, 1902, Principles of Political Economy, London - New York - Bombay.

Mirbach, Gunther Freiherr von, 1987, Grundgesetz und Wertungswidersprüche zwischen Ehegattenunterhalts- und Steuerrecht. Zur steuerrechtlichen Benachteiligung dauernd getrennt lebender und geschiedener Ehegatten, Steuer und Wirtschaft, 64, 319-326.

MIT-Rentenkommission, 1988, Kommissionsbericht, in: Christian SchwarzSchilling (Hrsg.), Langfristig sichere Rente. Ein Weg zur Erfüllung des Generationen-Vertrages, Bonn, 1-151.

Mitschke, Joachim, 1976, Über die Eignung von Einkommen, Konsum und Vermögen als Bemessungsgrundlagen der direkten Besteuerung, Diss. Saarbrücken, Berlin.

Mitschke, Joachim, 1980, Lebenseinkommensbesteuerung durch interperiodischen Progressionsausgleich, Steuer und Wirtschaft, 57, 122-134.

Mitschke, Joachim, 1985, Steuer- und Transferordnung aus einem Guß. Entwurf einer Neugestaltung der direkten Steuern und Sozialtransfers in der Bundesrepublik Deutschland, Baden-Baden.

Möller, Hans, 1950, Begriff und Einteilung der Versicherung, in: Verein für Versicherungswissenschaft und Praxis in Hessen (Hrsg.), Jahrbuch 1950, Frankfurt/Main, 25-38.

Müller, Wolfgang, 1988, Das Versicherungsprinzip - Zum Gefahrengemeinschaftsmythos in Versicherungstheorie und -praxis, in: Gabriele Rolf, P. Bernd Spahn und Gert Wagner (Hrsg.), Sozialvertrag und Sicherung. Zur ökonomischen Theorie staatlicher Versicherungs- und Umverteilungssysteme, Frankfurt/Main - New York, 129-146.

Müssener, Ingo, 1991, Vereinigte Staaten von Amerika, in: Annemarie Mennel (Hrsg.), Steuern in Europa, USA, Kanada und Japan, Loseblattausgabe, Herne - Berlin, 23. Lieferung.

Munnell, Alicia H., 1980, The Couple versus the Individual under the Federal Personal Income Tax, in: Henry J. Aaron und Michael J. Boskin (Hrsg.), The Economics of Taxation, Washington, 247-278.

Musgrave, Peggy B., 1981, Women and Taxation, in: Karl W. Roskamp und Francesco Forte (Hrsg.), Reforms of Tax Systems, Proceedings of the 35th Congress of the International Institute of Public Finance at Taormina 1979, Detroit, 341-354. 
Musgrave, Richard A., 1968a, In Defense of an Income Concept, in: Boris I. Bittker u.a., A Comprehensive Income Tax Base? A Debate, Branford, 62-80.

Musgrave, Richard A., 1968b, Rejoinder, in: Boris I. Bittker u.a., A Comprehensive Income Tax Base? A Debate, Branford, 114-116.

Musgrave, Richard A., 1985, Death and Taxes, in: H. Hanusch, K.W. Roskamp und J. Wiseman (Hrsg.), Staat und Politische Ökonomie heute, Horst Claus Recktenwald zum 65. Geburtstag, Stuttgart - New York, 149-155.

Musgrave, Richard A., 1986, ET, OT and SBT, in: Ders., Public Finance in a Democratic Society, Brighton, Bd.1, 260-273.

Musgrave, Richard A. / Musgrave, Peggy B. / Kullmer, Lore, 1988, Die öffentlichen Finanzen in Theorie und Praxis, Bd.2, 4.Aufl., Tübingen.

Naust, Hermann, 1991/92, Konsumorientierte Steuerreform. Zum Heidelberger Konsumsteuerkongreß, Finanzarchiv, N.F.49, 501-533.

Nell-Breuning, Oswald von, 1978, Vertrag zwischen drei Generationen, Wirtschaftswoche, 32, Nr.23 vom 2.6.1978, 77-80.

Neumark, Fritz, 1947, Theorie und Praxis der modernen Einkommensbesteuerung, Bern.

Neumark, Fritz, 1961a, Probleme der Steuerprogression, in: Ders., Wirtschafts- und Finanzprobleme des Interventionsstaates, Tübingen, 369390.

Neumark, Fritz, 1961b, Probleme der Allgemeinen Einkommenstheorie, in: Ders., Wirtschafts- und Finanzprobleme des Interventionsstaates, Tübingen, 23-51.

Neumark, Fritz, 1970, Grundsätze gerechter und ökonomisch rationaler Steuerpolitik, Tübingen.

Neumark, Fritz, 1977, Steuern I: Grundlagen, in: Willi Albers u.a. (Hrsg.), Handwörterbuch der Wirtschaftswissenschaft, Bd.7, Stuttgart u.a.O., 295-309.

Neumark, Fritz, 1984, Chancen einer Schumpeter-Renaissance, in: Dieter Bös und Hans-Dieter Stolper (Hrsg.), Schumpeter oder Keynes? Zur Wirtschaftspolitik der neunziger Jahre, Berlin u.a.O., 45-65.

Oberhauser, Alois, 1985, Die Ungereimtheiten des dualen Systems, Sozialer Fortschritt, 34, 14-20.

Oberhauser, Alois, 1989, Familie und Haushalt als Transferempfänger. Situation, Mängel und Reformansätze, Frankfurt/Main - New York.

Obstfelder, Volkmar G. von, 1976, Individualbesteuerung oder Haushaltsbesteuerung unter besonderer Berücksichtigung des Ehegatten-Splitting, Frankfurt/Main - Bern.

OECD, 1988a, The Taxation of Fringe Benefits, Paris. 
OECD, 1988b, The Taxation of Net Wealth, Capital Transfers and Capital Gains of Individuals, Paris.

Oehsen, Johann Hermann von, 1982, Optimale Besteuerung, Frankfurt/Main Bern.

Oeter, Ferdinand, 1967, Familienlastenausgleich oder gerechte soziale Lastenverteilung?, Sozialer Fortschritt, 16, 204-207.

Osthövener, Claus, 1971, Das "Wesen der Ehe" in der Sicht des Bundesfinanzhofs, Finanz-Rundschau, 26, 57-60.

o.V., 1991, Der Familienlastenausgleich muß reformiert werden, Soziale Sicherheit, 40, 17-19.

Paqué, Karl-Heinz, 1986, Philanthropie und Steuerpolitik. Eine ökonomische Analyse der Förderung privater Wohltätigkeit, Tübingen.

Pauly, Mark V., 1986, Taxation, Health Insurance, and Market Failure in the Medical Economy, Journal of Economic Literature, 24, 629-675.

Paul, Christine, 1990, Ehescheidungen 1989, Wirtschaft und Statistik, 837840.

Pechman, Joseph A., 1966, Federal Tax Policy, Washington.

Pechman, Joseph A., 1968, Comprehensive Income Taxation: A Comment, in: Boris I. Bittker u.a., A Comprehensive Income Tax Base? A Debate, Branford, 81-85.

Pechman, Joseph A., 1986, The Rich, the Poor, and the Taxes They Pay, Brighton.

Peffekoven, Rolf, 1971/72, Freibetrag oder Steuerkredit?, Finanzarchiv, N.F.30, 392-417.

Peffekoven, Rolf, 1980, Persönliche allgemeine Ausgabensteuer, in: Fritz Neumark, Norbert Andel und Heinz Haller (Hrsg.), Handbuch der Finanzwissenschaft, 3.Aufl., Bd.2, Tübingen, 417-452.

Petersen, Hans-Georg, 1977, Personelle Einkommensbesteuerung und Inflation. Eine theoretisch-empirische Analyse der Lohn- und veranlagten Einkommensteuer in der Bundesrepublik Deutschland, Frankfurt/Main Bern - Las Vegas.

Petersen, Hans-Georg, 1982, Aufkommens- und Verteilungswirkungen alternativer Rentenanpassungs- und -besteuerungsverfahren, Finanzarchiv, N.F.40, 385-417.

Pezzer, H.-J., 1989, Familienbesteuerung und Grundgesetz, Steuer und Wirtschaft, 66, 219-226.

Pfaff, Martin / Pfaff, Anita, 1971, Grants Economics: An Evaluation of Government Policies, Public Finance, 26, 275-303.

Phelps, Charles E., 1984/85, Taxing Health Insurance: How Much is Enough?, Contemporary Policy Issues, 3, 47-54. 
Pohmer, Dieter, 1968, Einige Gesichtspunkte zur Familienbesteuerung. Die Behandlung der Steuerpflichtigen mit Kindern im Einkommensteuerrecht, Finanzarchiv, N.F.27, 139-173.

Pohmer, Dieter, 1985, Einige finanzpolitische Aspekte der Familienpolitik unter besonderer Berücksichtigung von Problemen der Familienbesteuerung, in: H. Hanusch, K.W. Roskamp und J. Wiseman (Hrsg.), Staat und Politische Ökonomie heute, Horst Claus Recktenwald zum 65. Geburtstag, Stuttgart - New York, 237-260.

Pollak, Helga, 1979, Welche Auswirkungen haben die von der Kommission vorgesehenen Modelle im Steuerrecht, und welche ergänzenden Maßnahmen sind im Steuerrecht erforderlich?, in: Sachverständigenkommission für die soziale Sicherung der Frau und der Hinterbliebenen, Gutachten, Anlageband 2, Stuttgart u.a.O., 257-277.

Pollak, Helga, 1987, Gibt es einen Wandel in der Einkommensteuer?, in: Cay Folkers, Helga Pollak und Herbert Timm, Wandlungen der Besteuerung, hrsg. von Karl Häuser, Berlin.

Pommerehne, Werner W., 1979, Gebundene vs. freie Geldtransfers: Eine Fallstudie, in: Carl Christian von Weizsäcker (Hrsg.), Staat und Wirtschaft, Berlin, 147-164.

President of the United States, 1985, The President's Tax Proposals to the Congress for Fairness, Growth, and Simplicity, Washington.

Prest, Alan R., 1968, The Budget and Interpersonal Distribution, Public Finance, 23, 80-98.

Prinz, Aloys, 1986, Besteuerung von Transfers auf Sozialhilfeniveau bei temporärer Arbeitslosigkeit?, Finanzarchiv, N.F.44, 110-122.

Prinz, Ulrich, 1988, Kommentierung von § 9 EStG, in: Herrmann / Heuer / Raupach, Einkommensteuer- und Körperschaftsteuergesetz. Kommentar, Lieferung 158.

Pump, H., 1987, Zusammenveranlagung bei der Einkommensteuer - Aufteilungsbescheid und Aufrechnung, Die Information über Steuer und Wirtschaft, 41, 481-486.

Ramm, Thilo, 1985, Familienrecht, Bd.I: Recht der Ehe, München.

Rehfeld, Uwe / Luckert, Hilmar, 1989, Die versicherungsfremden Leistungen der Rentenversicherung - Eine Schätzung von Häufigkeiten und Volumen, Deutsche Rentenversicherung, 42-71.

Reuter, Hans-Peter, 1987, Die Lebensversicherung im Steuerrecht, 7.Aufl., Herne - Berlin.

Richter, Wolfram F., 1987, Neutrale Ertragsanteilsbesteuerung von Renten, Deutsche Rentenversicherung, 662-685.

Rippe, Wolfgang, 1981, Freiwillige Übertragungen als Problem der Transferökonomie, Baden-Baden. 
Rössler, Rudolf / Troll, Max, 1989, Bewertungsgesetz und Vermögensteuergesetz, 15.Aufl., München.

Rolph, Earl R., 1948, The Concept of Transfers in National Income Estimates, Quarterly Journal of Economics, 62, 327-361.

Royal Commission on Taxation, 1966, Report, Ottawa.

Rüstow, Alexander, 1950, Das Versagen des Wirtschaftsliberalismus, (Godesberg).

Ruland, Franz, 1973, Familiärer Unterhalt und Leistungen der sozialen Sicherheit. Zugleich ein Beitrag zur Reform der sozialen Sicherung der Ehegatten und zur Reform des Familienlastenausgleichs, Berlin.

Ruland, Franz, 1983, Möglichkeiten und Grenzen einer Annäherung der Beamtenversorgung an die gesetzliche Rentenversicherung, Anlagenband B zum Gutachten der Sachverständigenkommission Alterssicherungssysteme vom 19. November 1983, Stuttgart u.a.O.

Ruppe, Hans Georg, 1990, Einführung zum Einkommensteuergesetz, in: Herrmann / Heuer / Raupach, Einkommensteuer- und Körperschaftsteuergesetz. Kommentar, 162. Lieferung.

Sachverständigenrat zur Begutachtung der gesamtwirtschaftlichen Entwicklung, 1991, Die wirtschaftliche Integration in Deutschland. Perspektiven Wege - Risiken, Jahresgutachten 1991/92, Stuttgart.

Schäfer, Dieter, 1980, Lebensstandardprinzip und Steuerpflicht bei Sozialleistungen, in: Klaus Schenke und Winfried Schmähl (Hrsg.), Alterssicherung als Aufgabe für Wissenschaft und Politik, Helmut Meinhold zum 65. Geburtstag, Stuttgart u.a.O., 455-477.

Schanz, Georg, 1896, Der Einkommensbegriff und die Einkommensteuergesetze, Finanz-Archiv, 13, 1-81.

Scherf, Wolfgang, 1992, Der Regierungsentwurf zur Neuregelung des Familienlastenausgleichs, Sozialer Fortschritt, 41, 69-71.

Schlicht, Ekkehart, 1985, The Shadow Economy and Morals: A Note, in: Wulf Gaertner und Alois Wenig (Hrsg.), The Economics of the Shadow Economy, Berlin u.a.O., 265-271.

Schlieper, Ulrich, 1980, Externe Effekte, in: Willi Albers u.a. (Hrsg.), Handwörterbuch der Wirtschaftswissenschaft, Bd.2, Stuttgart u.a.O., 524-530.

Schlüter, Wilfried, 1991, BGB Familienrecht, 5.Aufl., Heidelberg.

Schmähl, Winfried, 1981, Elemente einer künftigen Rentenreform: Veränderungen der Anpassung und Besteuerung von Renten - Analysen und Vorschläge -, in: Sozialbeirat, Langfristige Probleme der Alterssicherung in der Bundesrepublik Deutschland, Bd.3: Einzelgutachten, (Bonn), 83357.

Schmähl, Winfried, 1986, Teilbesteuerung versus Vollbesteuerung von Renten, Deutsche Rentenversicherung, 101-128. 
Schmidt, Henning / Frank, Udo / Müller-Rohr, Iris, 1985, Kritische Bemerkungen zum System des Kinderlastenausgleichs - zugleich ein Vorschlag zur Neugestaltung der gesetzlichen Rentenversicherung, Finanzarchiv, N.F.43, 28-66.

Schmidt, Kurt, 1988, Notizen zur Steuerreform, Finanzarchiv, N.F.46, 193213.

Schneider, Dieter, 1971, Gewinnermittlung und steuerliche Gerechtigkeit, Schmalenbachs Zeitschrift für betriebswirtschaftliche Forschung, 23, 352-394.

Schneider, Dieter, 1978, Steuerbilanzen. Rechnungslegung als Messung steuerlicher Leistungsfähigkeit, Wiesbaden.

Schneider, Dieter, 1979, Bezugsgrößen steuerlicher Leistungsfähigkeit und Vermögensbesteuerung, Finanzarchiv, N.F.37, 26-49.

Schneider, Dieter, 1984, Leistungsfähigkeitsprinzip und Abzug von der Bemessungsgrundlage, Steuer und Wirtschaft, 61, 356-367.

Schreiber, Wilfrid, 1955, Existenzsicherheit in der industriellen Gesellschaft, Köln.

Schulte, Bernd / Trenk-Hinterberger, Peter, 1986, Sozialhilfe, 2.Aufl., Heidelberg.

Schwab, Dieter, 1989, Zugewinnausgleich, in: Ders. (Hrsg.), Handbuch des Scheidungsrechts, 2.Aufl., München, 1075-1169.

Schwab, Dieter, 1991, Familienrecht, 6.Aufl., München.

Schwab, Hartmut, 1988, Die betriebliche Altersversorgung - ein praktisches Modell für die Planung und Gestaltung, Hamburg.

Schwab, Karl Heinz, 1987, Sachenrecht, 21.Aufl., München.

Schwebler, Robert, 1988, Lebensversicherung, in: Dieter Farny u.a. (Hrsg.), Handwörterbuch der Versicherung, Karlsruhe, 417-425.

Segalen, Martine, 1990, Die Familie. Geschichte, Soziologie, Anthropologie, Frankfurt/Main - New York.

Seidl, Christian, 1983, Gerechtigkeit und Besteuerung unter besonderer Berücksichtigung der Optimalsteuertheorie, in: Dieter Pohmer (Hrsg.), Zur optimalen Besteuerung, Berlin, 163-259.

Siebeck, Theo, 1989, Sozialversicherung und Beamtensicherung. Hergebrachte Grundsätze - gegenwärtige Probleme, Sankt Augustin.

Simon, 1978, Abweichende Meinung des Richters Dr. Simon zum Beschluß des Ersten Senats des Bundesverfassungsgerichts vom 11. Oktober 1977, in: Bundesverfassungsgericht, Entscheidungen, Bd.47, Tübingen, 34-46.

Simons, Henry C., 1938, Personal Income Taxation. The Definition of Income as a Problem of Fiscal Policy, Chicago - London (Nachdruck 1980). 
Sinn, Hans-Werner, 1988, Die Grenzen des Versicherungsstaates - Theoretische Bemerkungen zum Thema Einkommensverteilung, Versicherung und Wohlfahrt, in: Gabriele Rolf, P. Bernd Spahn und Gert Wagner (Hrsg.), Sozialvertrag und Sicherung. Zur ökonomischen Theorie staatlicher Versicherungs- und Umverteilungssysteme, Frankfurt/Main - New York, 65-84.

Smeeding, Timothy M., 1984, Approaches to Measuring and Valuing In-Kind Subsidies and the Distribution of Their Benefits, in: Marilyn Moon (Hrsg.), Economic Transfers in the United States, Chicago - London, 139-171.

Söffing, Günter, 1979, Möglichkeiten und Grenzen einer Verlagerung von Einkünften auf die folgende Generation, in: Steuerberater-Jahrbuch 1978/79, Köln, 301-374.

Söhn, Hartmut, 1985, Sonderausgaben ( $\$ 10$ EStG) und Besteuerung nach der Leistungsfähigkeit, Steuer und Wirtschaft, 62, 395-407.

Söhn, Hartmut, 1986, Abzug von Rentenversicherungsbeiträgen als Sonderausgaben ( $\$ 10$ Abs.1 Nr.2 EStG) und Ertragsanteilsbesteuerung von Leibrenten (§ 22 Nr.1 Satz 3 Buchstabe a EStG), Steuer und Wirtschaft, 63, 324-334.

Söhn, Hartmut, 1988, Verfassungsrechtliche Aspekte der Besteuerung nach der subjektiven Leistungsfähigkeit im Einkommensteuerrecht: Zum persönlichen Existenzminimum, Finanzarchiv, N.F.46, 154-171.

Sommer, Bettina, 1987, Kranken- und Rentenversicherungsschutz der Bevölkerung. Ergebnis des Mikrozensus Juni 1985, Wirtschaft und Statistik, 252259.

Sozialenquête-Kommission, 1966, Soziale Sicherung in der Bundesrepublik Deutschland, Stuttgart u.a.O.

Spahn, P. Bernd, 1989, Agenda für eine Reform der Haushaltsbesteuerung um die Jahrtausendwende, Sonderforschungsbereich 3 der Johann Wolfgang Goethe-Universität Frankfurt und der Universität Mannheim, Arbeitspapier Nr. 279, Frankfurt/Main - Mannheim.

Spahn, P. Bernd / Kaiser, Helmut, 1988, Soziale Sicherheit als öffentliches Gut? - Zur Problematik der Steuerfinanzierung sozialer Sicherung, in: Gabriele Rolf, P. Bernd Spahn und Gert Wagner (Hrsg.), Sozialvertrag und Sicherung. Zur ökonomischen Theorie staatlicher Versicherungs- und Umverteilungssysteme, Frankfurt/Main - New York, 195-218.

Statistisches Bundesamt (Hrsg.), 1989, Volkswirtschaftliche Gesamtrechnungen, Fachserie 18, Reihe 1.3: Konten und Standardtabellen 1988, Stuttgart.

Statistisches Bundesamt (Hrsg.), 1991, Statistisches Jahrbuch 1991 für das vereinte Deutschland, Wiesbaden. 
Steuerreformkommission, 1971, Gutachten der Steuerreformkommission 1971, Schriftenreihe des Bundesministeriums der Finanzen, Heft 17, Bonn.

Stiglitz, Joseph E., 1982, Utilitarianism and Horizontal Equity, Journal of Public Economics, 18, 1-33.

Stobbe, Alfred, 1989, Volkswirtschaftliches Rechnungswesen, 7.Aufl., Berlin u.a.O.

Tanzi, Vito, 1980, Inflation and the Personal Income Tax: An International Perspective, Cambridge u.a.O.

Tinbergen, Jan, 1978, Einkommensverteilung. Auf dem Weg zu einer neuen Einkommensgerechtigkeit, Wiesbaden.

Tipke, Klaus, 1980, Bezüge und Abzüge im Einkommensteuerrecht, Steuer und Wirtschaft, 57, 1-11.

Tipke, Klaus, 1984, Zur Reform der Familienbesteuerung, Steuer und Wirtschaft, 61, 127-128.

Tipke, Klaus / Lang, Joachim, 1991, Steuerrecht. Ein systematischer Grundriß, 13.Aufl., Köln.

Transfer-Enquête-Kommission, 1979, Zur Einkommenslage der Rentner. Zwischenbericht der Kommission, Bonn.

Transfer-Enquête-Kommission, 1981, Das Transfersystem in der Bundesrepublik Deutschland, Stuttgart u.a.O.

Vickrey, William, 1947, Agenda for Progressive Taxation, New York.

Vickrey, William, 1959, Averaging of Income for Income-Tax Purposes, in: American Economic Association (Hrsg.), Readings in the Economics of Taxation, London, 77-92.

Vogel, Klaus, 1984, Zwangsläufige Aufwendungen - besonders Unterhaltsaufwendungen - müssen realitätsgerecht abziehbar sein, Steuer und Wirtschaft, 61, 197-203.

Wagner, Gert, 1984, Umverteilung in der gesetzlichen Rentenversicherung, Frankfurt/Main - New York.

Wagner, Gert, 1985a, Zur Meßbarkeit eines versicherungsgemäßen Risikoausgleichs und der Umverteilung in der gesetzlichen Rentenversicherung, in: Winfried Schmähl (Hrsg.), Versicherungsprinzip und soziale Sicherung, Tübingen, 141-203.

Wagner, Gert, 1985b, Interpersonelle Umverteilung in der gesetzlichen Rentenversicherung, Wirtschaftsdienst, 65, 190-196.

Walzer, Klaus, 1987, Steuergerechtigkeit. Eine entscheidungstheoretische Interpretation, Berlin.

Weyers, Gerd / Oberhauser, Alois, 1984, Kumulationsabbau bei den Sozialleistungen, Berlin. 
Wiese, Leopold von, 1928, Das Prinzip der Progression in der Einkommensteuer, in: Hans Teschemacher (Hrsg.), Beiträge zur Finanzwissenschaft, Festgabe für Georg von Schanz zum 75. Geburtstag, Tübingen, Bd.2, 261-272.

Winterstein, Helmut, 1965, Interpersonaler und intertemporaler Einkommensausgleich - Ein Beitrag zur Umverteilungsterminologie, Sozialer Fortschritt, 14, 36-38.

Wissenschaftlicher Beirat beim Bundesministerium der Finanzen, 1967, Gutachten zur Reform der direkten Steuern (Einkommensteuer, Körperschaftsteuer, Vermögensteuer und Erbschaftsteuer) in der Bundesrepublik Deutschland vom 11. Februar 1967, abgedruckt in: Ders., Entschließungen, Stellungnahmen und Gutachten 1949-1973, Tübingen 1974, 326400 .

Wissenschaftlicher Beirat beim Bundesministerium der Finanzen, 1971, Gutachten zur Neugestaltung und Finanzierung von Alterssicherung und Familienlastenausgleich vom 1. Juni 1971, abgedruckt in: Ders., Entschließungen, Stellungnahmen und Gutachten 1949-1973, Tübingen 1974, 475-531.

Wissenschaftlicher Beirat beim Bundesministerium der Finanzen, 1986, Gutachten zur einkommensteuerlichen Behandlung von Alterseinkünften vom 15. Februar 1986, abgedruckt in: Ders., Gutachten und Stellungnahmen 1974-1987, Tübingen 1988, 513-557.

Wissenschaftlicher Beirat beim Bundesministerium der Finanzen, 1988, Stellungnahme zum vorgesehenen "Abbau von Steuersubventionen und Sonderregelungen", BMF-Dokumentation 1/88 vom 11.1.1988.

Wissenschaftlicher Beirat beim Bundesministerium der Finanzen, 1989, Die Einheitsbewertung in der Bundesrepublik Deutschland - Mängel und Alternativen, Schriftenreihe des Bundesministeriums der Finanzen, Heft 41, Bonn.

Wolff, Volker, 1988, Trauerfall. Wie der Beruf die Lebenserwartung beeinflußt, Capital, 27, Nr.10 vom 30.9.1988, 165-166.

Zeppernick, Ralf, 1974, Untersuchungen zum Familienlastenausgleich, Köln.

Zeppernick, Ralf, 1986, Transfer-Einkommen und Einkommensverteilung, Berlin.

Zimmermann, Horst, 1989, Kindergeld, Kinderfreibetrag oder Familiensplitting?, Wirtschaftsdienst, 69, 149-153.

Zitzelsberger, Heribert, 1984, Reformüberlegungen zur Besteuerung der Alterseinkommen, Deutsche Steuer-Zeitung, 72, 467-475.

Zmarzlik, Johannes / Zipperer, Manfred / Viethen, Hans Peter, 1986, Mutterschutzgesetz, Mutterschaftshilfe, Erziehungsgeld, Erziehungsurlaub, 5.Aufl., Köln u.a.O. 


\section{VERWENDETE GESETZESTEXTE}

Abgabenordnung (AO) vom 16.3.1976, zuletzt geändert durch Gesetz vom 21.12.1992.

Arbeitsförderungsgesetz (AFG) vom 25.6.1969, zuletzt geändert durch Verordnung vom 26.2.1993.

Bewertungsgesetz (BewG) vom 1.2.1991, zuletzt geändert durch Gesetz vom 9.11.1992.

Bürgerliches Gesetzbuch (BGB) vom 18.8.1896, zuletzt geändert durch Gesetz vom 4.12.1992.

Bundesausbildungsförderungsgesetz (BAföG) vom 6.6.1983, zuletzt geändert durch Gesetz vom 21.12.1992.

Bundeserziehungsgeldgesetz (BErzGG) vom 21.1.1992, zuletzt geändert durch Gesetz vom 11.1.1993.

Bundeskindergeldgesetz (BKGG) vom 30.1.1990, zuletzt geändert durch Gesetz vom 11.1.1993.

Bundessozialhilfegesetz (BSHG) vom 10.1.1991, zuletzt geändert durch Gesetz vom 16.2.1993.

Einkommensteuergesetz (EStG) vom 7.9.1990, zuletzt geändert durch Gesetz vom 21.12.1992.

Grundgesetz (GG) vom 23.5.1949, zuletzt geändert durch Gesetz vom 21.12.1992.

Hessisches Gesetz zum Abbau der Fehlsubventionierung im Wohnungswesen vom 25.2.1992.

Lohnsteuerdurchführungsverordnung (LStDV) vom 10.10.1989, zuletzt geändert durch Gesetz vom 25.2.1992.

Regelsatzverordnung (Verordnung zur Durchführung des $\S 22$ des Bundessozialhilfegesetzes) vom 20.7.1962, zuletzt geändert durch Verordnung vom 7.10.1991.

Sozialgesetzbuch, zuletzt geändert durch Gesetz vom 21.12.1992.

Versicherungsvertragsgesetz (VVG) vom 30.5.1908, zuletzt geändert durch Gesetz vom 17.12.1990.

Wohngeldgesetz (WoGG) vom 1.2.1993.

Wohngeldverordnung (WoGVO) vom 30.9.1992.

Zivilprozeßordnung (ZPO) vom 12.9.1950, zuletzt geändert durch Gesetz vom 11.1.1993.

Zweites Wohnungsbaugesetz vom 14.8.1990, zuletzt geändert durch Gesetz vom 27.7.1992. 


\section{FINANZWISSENSCHAFTLICHE SCHRIFTEN}

Band 1 Werner Steden: Finanzpolitik und Einkommensverteilung. Ein Wachstums- und Konjunkturmodell der Bundesrepublik Deutschland. 1979.

Band 2 Rainer Hagemann: Kommunale Finanzplanung im föderativen Staat. 1976.

Band 3 Klaus Scherer: Maßstäbe zur Beurteilung von konjunkturellen Wirkungen des öffentlichen Haushalts. 1977.

Band 4 Brita Steinbach: "Formula Flexibility" - Kritische Analyse und Vergleich mit diskretionärer Konjunkturpolitik. 1977.

Band 5 Hans-Georg Petersen: Personelle Einkommensbesteuerung und Inflation. Eine theoretisch-empirische Analyse der Lohn- und veranlagten Einkommensteuer in der Bundesrepublik Deutschland. 1977.

Band 6 Friedemann Tetsch: Raumwirkungen des Finanzsystems der Bundesrepublik Deutschland. Eine Untersuchung der Auswirkungen der Finanzreform von 1969 auf die Einnahmenposition der untergeordneten Gebietskörperschaften und ihrer regionalpolitischen Zieladäquanz. 1978.

Band 7 Wilhelm Ptähler: Normative Theorie der fiskalischen Besteuerung. Ein methodologischer und theoretischer Beitrag zur Integration der normativen Besteuerungstheorie in der Wohlfahrtstheorie. 1978.

Band 8 Wolfgang Wiegard: Optimale Schattenpreise und Produktionsprogramme für öffentliche Unternehmen. Second-Best Modelle im finanzwirtschaftlichen Staatsbereich. 1978.

Band 9 Hans P. Fischer: Die Finanzierung des Umweltschutzes im Rahmen einer rationalen Umweltpolitik. 1978.

Band 10 Rainer Paulenz: Der Einsatz finanzpolitischer Instrumente in der Forschungs- und Entwicklungspolitik. 1978.

Band 11 Hans-Joachim Hauser: Verteilungswirkungen der Staatsverschuldung. Eine kreislauftheoretische Inzidenzbetrachtung. 1979.

Band 12 Gunnar Schwarting: Kommunale Investitionen. Theoretische und empirische Untersuchungen der Bestimmungsgründe kommunaler Investitionstätigkeit in NordrheinWestfalen 1965-1972. 1979.

Band 13 Hans-Joachim Conrad: Stadt-Umland-Wanderung und Finanzwirtschaft der Kernstädte. Amerikanische Erfahrungen, grundsätzliche Zusammenhänge und eine Fallstudie für das Ballungsgebiet Frankfurt am Main. 1980.

Band 14 Cay Folkers: Vermögensverteilung und staatliche Aktivität. Zur Theorie distributiver Prozesse im Interventionsstaat. 1981.

Band 15 Helmut Fischer: US-amerikanische Exporttörderung durch die DISC-Gesetzgebung. 1981.

Band 16 Günter Ott: Einkommensumverteilungen in der gesetzlichen Krankenversicherung. Eine quantitative Analyse. 1981.

Band 17 Johann Hermann von Oehsen: Optimale Besteuerung. (Optimal Taxation). 1982.

Band 18 Richard Kössler: Sozialversicherungsprinzip und Staatszuschüsse in der gesetzlichen Rentenversicherung. 1982.

Band 19 Hinrich Steffen: Zum Handlungs- und Entscheidungsspielraum der kommunalen Investitionspolitik in der Bundesrepublik Deutschland. 1983.

Band 20 Manfred Scheuer: Wirkungen einer Auslandsverschuldung des Staates bei flexiblen Wechselkursen. 1983. 
Band 21 Christian Schiller: Staatsausgaben und crowding-out-Effekte. Zur Effizienz einer Finanzpolitik keynesianischer Provenienz. 1983.

Band 22 Hannelore Weck: Schattenwirtschaft: Eine Möglichkeit zur Einschränkung der öffentlichen Verwaltung? Eine ökonomische Analyse. 1983.

Band 23 Wolfgang Schmitt: Steuern als Mittel der Einkommenspolitik. Eine Ergänzung der Stabilitätspolitik? 1984.

Band 24 Wolfgang Laux: Erhöhung staatswirtschaftlicher Effizienz durch budgetäre Selbstbeschränkung? Zur Idee einer verfassungsmäßig verankerten Ausgabengrenze. 1984.

Band 25 Brita Steinbach-van der Veen: Steuerinzidenz. Methodologische Grundlagen und empirisch-statistische Probleme von Länderstudien. 1985.

Band 26 Albert Peters: Ökonomische Kriterien für eine Aufgabenverteilung in der Marktwirtschaft. Eine deskriptive und normative Betrachtung für den Allokationsbereich. 1985.

Band 27 Achim Zeidler: Möglichkeiten zur Fortsetzung der Gemeindefinanzreform. Eine theoretische und empirische Analyse. 1985.

Band 28 Peter Bartsch: Zur Theorie der längerfristigen Wirkungen 'expansiver' Fiskalpolitik. Eine dynamische Analyse unter besonderer Berücksichtigung der staatlichen Budgetbeschränkung und ausgewählter Möglichkeiten der öffentlichen Defizitfinanzierung. 1986.

Band 29 Konrad Beiwinkel: Wehrgerechtigkeit als finanzpolitisches Verteilungsproblem. Möglichkeiten einer Kompensation von Wehrungerechtigkeit durch monetäre Transfers. 1986.

Band 30 Wolfgang Kitterer: Effizienz- und Verteilungswirkungen des Steuersystems. 1986.

Band 31 Heinz Dieter Hessler: Theorie und Politik der Personalsteuern. Eine Kritik ihrer Einkommens- und Vermögensbegriffe. 1993.

Band 32 Wolfgang Scherf: Die beschäftigungspolitische und fiskalische Problematik der Arbeitgeberbeiträge zur Rentenversicherung. Eine Auseinandersetzung mit der Kritik an der lohnbezogenen Beitragsbemessung. 1987.

Band 33 Andreas Mästle: Die Steuerunion. Probleme der Harmonisierung spezifischer Gütersteuern. 1987.

Band 34 Günter Ott: Internationale Verteilungswirkungen im Finanzausgleich der Europäischen Gemeinschaften. 1987.

Band 35 Heinz Haller: Zur Frage der zweckmäßigen Gestalt gemeindlicher Steuern. Ein Diskussionsbeitrag zur Gemeindesteuerreform. 1987.

Band 36 Thomas Kuhn: Schlüsselzuweisungen und fiskalische Ungleichheit. Eine theoretische Analyse der Verteilung von Schlüsselzuweisungen an Kommunen. 1988.

Band 37 Walter Hahn: Steuerpolitische Willensbildungsprozesse in der Europäischen Gemeinschaft. Das Beispiel der Umsatzssteuer-Harmonisierung. 1988.

Band 38 Ulrike Hardt: Kommunale Finanzkraft. Die Problematik einer objektiven Bestimmung kommunaler Einnahmemöglichkeiten in der gemeindlichen Haushaltsplanung und im kommunalen Finanzausgleich. 1988.

Band 39 Jochen Michaelis: Optimale Finanzpolitik im Modell überlappender Generationen. 1989

Band 40 Bernd Raffelhüschen: Anreizwirkungen der sozialen Alterssicherung. Eine dynamische Simulationsanalyse. 1989.

Band 41 Berend Diekmann: Die Anleihe- und Darlehenstransaktionen der Europäischen Gemeinschaften. 1990.

Band 42 Helmut Kaiser: Konsumnachfrage, Areitsangebot und optimale Haushaltsbesteuerung Theoretische Ergebnisse und mikroökonometrische Simulation für die Bundesrepublik Deutschland. 1990. 
Band 43 Rüdiger von Kleist: Das Gramm-Rudman-Hollings-Gesetz. Ein gescheiterter Versuch der Haushaltskonsolidierung. 1991.

Band 44 Rolf Hagedorn: Steuerhinterziehung und Finanzpolitik. Ein theoretischer Beitrag unter besonderer Berücksichtigung der Hinterziehung von Zinserträgen. 1991.

Band 45 Cornelia S. Behrens: Intertemporale Verteilungswirkungen in der gesetzlichen Krankenversicherung der Bundesrepublik Deutschland. 1991.

Band 46 Peter Saile: Ein ökonomischer Ansatz der Theorie der intermediären Finanzgewalten Die Kirchen als Parafisci. 1992.

Band 47 Peter Gottfried: Die verdeckten Effizienzwirkungen der Umsatzsteuer. Eine empirische allgemeine Gleichgewichtsanalyse. 1992.

Band 48 Andreas Burger: Umweltorientierte Beschäftigungsprogramme. Eine Effizienzanalyse am Beispiel des "Sondervermögens Arbeit und Umwelt". 1992.

Band 49 Jeanette Malchow: Die Zuordnung verteilungspolitischer Kompetenzen in der Europäischen Gemeinschaft. Eine Untersuchung aufgrund einer Fortentwicklung der ökonomischen Theorie des Föderalismus. 1992.

Band 50 Barbara Seidel: Die Einbindung der Bundesrepublik Deutschland in die Europäischen Gemeinschaften als Problem des Finanzausgleichs. 1992.

Band 51 Ralph Wiechers: Markt und Macht im Rundfunk. Zur Stellung der öffentlich-rechtlichen Rundfunkanstalten im dualen Rundfunksystem der Bundesrepublik Deutschland. 1992.

Band 52 Klaus Eckhardt: Probleme einer Umweltpolitik mit Abgaben. 1993.

Band 53 Oliver Schwarzkopf: Die Problematik unterschiedlicher Körperschaftsteuersysteme innerhalb der EG. 1993.

Band 54 Thorsten Giersch: Bergson-Wohlfahrtsfunktion und normative Ökonomie. 1993.

Band 55 Li-Fang Chou: Selbstbeteiligung bei Arzneimitteln aus ordnungspolitischer Sicht. Das Beispiel der Bundesrepublik Deutschland. 1993.

Band 56 Harald Schlee: Einkommensteuerliche Behandlung von Transferzahlungen. Zur Neuordnung der Familienbesteuerung sowie der Besteuerung von Versicherungsleistungen und Sozialtransfers. 1994. 